Nevada

Environmental

Restoration

Project

Corrective Action Decision

Document/Closure Report for

Corrective Action Unit 234: Mud Pits,

Cellars, and Mud Spills

Nevada Test Site, Nevada

Controlled Copy No.:

Revision No:: 0

May 2008

Approved for public release; further dissemination unlimited.

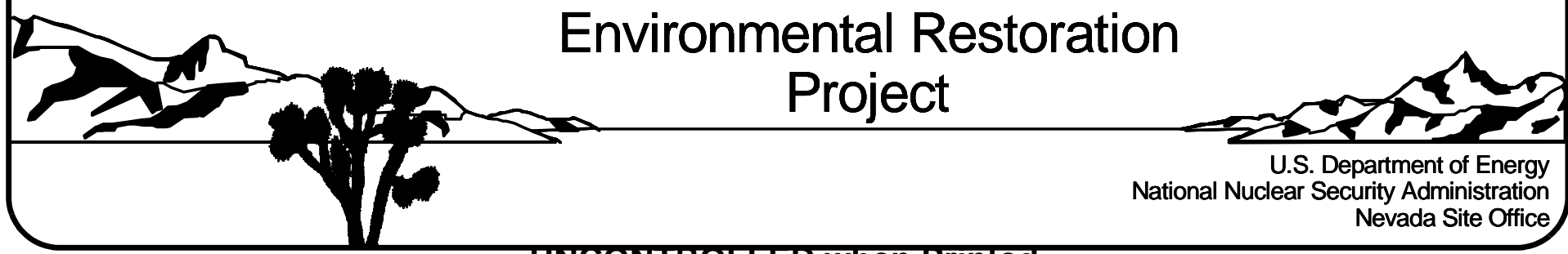


Available for public sale, in paper, from:

U.S. Department of Commerce

National Technical Information Service

5285 Port Royal Road

Springfield, VA 22161

Phone: 800.553 .6847

Fax: 703.605.6900

Email: orders@ntis.gov

Online ordering: http://www.ntis.gov/ordering.htm

Available electronically at http://www.osti.gov/bridge

Available for a processing fee to U.S. Department of Energy and its contractors, in paper, from:

U.S. Department of Energy

Office of Scientific and Technical Information

P.O. Box 62

Oak Ridge, TN 37831-0062

Phone: 865.576.8401

Fax: 865.576.5728

Email: reports@adonis.osti.gov

Reference herein to any specific commercial product, process, or service by trade name, trademark, manufacturer, or otherwise, does not necessarily constitute or imply its endorsement, recommendation, or favoring by the United States Government or any agency thereof or its contractors or subcontractors. 


\title{
CORRECTIVE ACTION DECISION DOCUMENT/ CLOSURE REPORT FOR CORRECTIVE ACTION UNIT 234: MUD PITS, CELLARS, AND MUD SPILLS NEVADA TEST SITE, NEVADA
}

\author{
U.S. Department of Energy \\ National Nuclear Security Administration \\ Nevada Site Office \\ Las Vegas, Nevada
}

Controlled Copy No.:

Revision No.: 0

May 2008

Approved for public release; further dissemination unlimited. 


\section{CORRECTIVE ACTION DECISION DOCUMENT/CLOSURE REPORT FOR \\ CORRECTIVE ACTION UNIT 234: MUD PITS, CELLARS, AND MUD SPILLS \\ NEVADA TEST SITE, NEVADA}

Approved by: /s/ Kevin J. Cabble

Kevin J. Cabble

Federal Sub-Project Director

Industrial Sites Sub-Project

Approved by: /s/ John B. Jones

John B. Jones

Acting Federal Project Director

Environmental Restoration Project 


\section{Table of Contents}

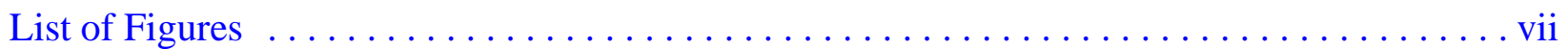

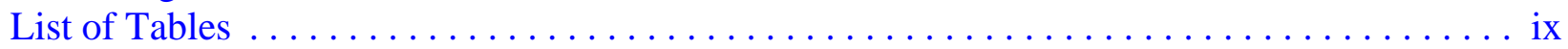

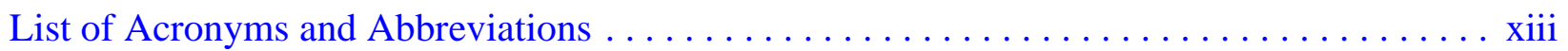

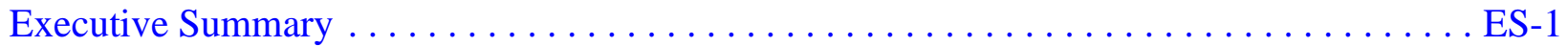

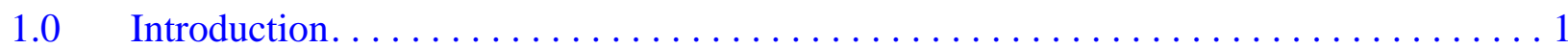

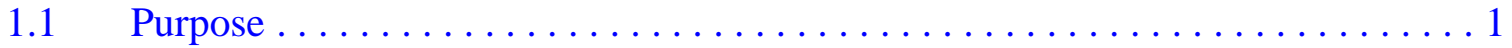

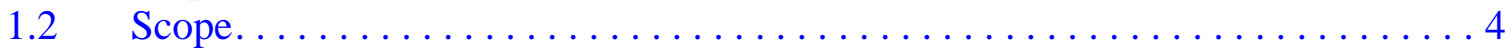

1.3 Corrective Action Decision Document/Closure Report Contents . . . . . . . . . . 5

1.3.1 Applicable Programmatic Plans and Documents .............6 6

1.3.2 Data Quality Assessment Summary .....................6 6

$2.0 \quad$ Corrective Action Investigation Summary . . . . . . . . . . . . . . . . . . 7

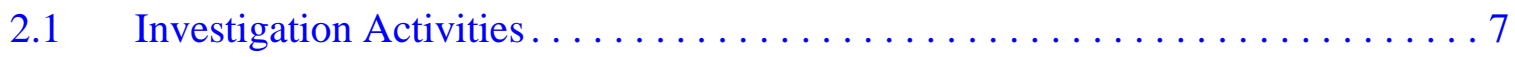

2.1.1 Area 2 Mud Plant \#1 (CAS 02-09-48) . . . . . . . . . . . . . 8

2.1.1.1 Radiological Survey $\ldots \ldots \ldots \ldots \ldots \ldots \ldots \ldots \ldots$

2.1.1.2 Visual Inspection $\ldots \ldots \ldots \ldots \ldots \ldots \ldots \ldots \ldots \ldots$

2.1.1.3 Field Screening $\ldots \ldots \ldots \ldots \ldots \ldots \ldots \ldots \ldots$

2.1.1.4 Sample Collection ....................... 9

2.1.1.5 Conceptual Site Model Validation ................ 9

2.1.2 Mud Dump Trenches (CAS 03-09-02) .................... 9

2.1.2.1 Radiological Survey $\ldots \ldots \ldots \ldots \ldots \ldots \ldots \ldots$

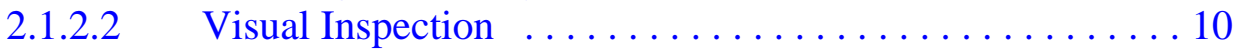

2.1.2.3 Field Screening . . . . . . . . . . . . . . . . . . . 10

2.1.2.4 Sample Collection ........................... 10

2.1.2.5 Conceptual Site Model Validation . . . . . . . . . . . . . 10

2.1.3 Mud Pit (CAS 12-09-01) ............................ 11

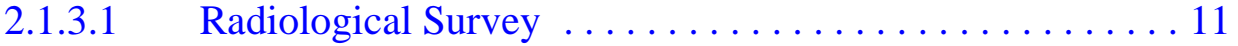

2.1.3.2 Visual Inspection $\ldots \ldots \ldots \ldots \ldots \ldots \ldots \ldots \ldots \ldots \ldots \ldots \ldots$

2.1.3.3 Field Screening . . . . . . . . . . . . . . . . . . . . 11

2.1.3.4 Sample Collection .......................... 11

2.1.3.5 Conceptual Site Model Validation ................. 11

2.1.4 Mud Pit (CAS 12-09-08) .......................... 12

2.1.4.1 Radiological Survey . . . . . . . . . . . . . . . . 12

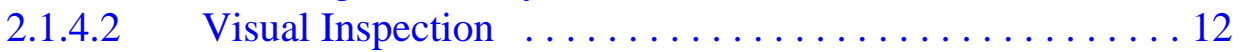

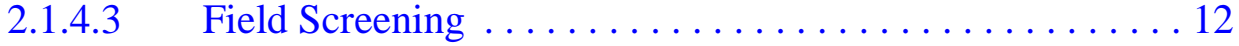

2.1.4.4 Sample Collection ....................... 12

2.1.4.5 Conceptual Site Model Validation .............. 13

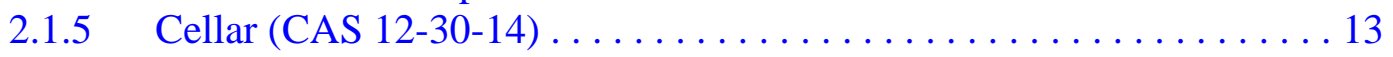

2.1.5.1 Radiological Survey ....................... 13 


\section{Table of Contents (Continued)}

2.1.5.2 Visual Inspection $\ldots \ldots \ldots \ldots \ldots \ldots \ldots \ldots \ldots \ldots$

2.1.5.3 Field Screening ........................... 13

2.1.5.4 Sample Collection .......................... 13

2.1.5.5 Conceptual Site Model Validation ................ 14

2.1.6 Summary of Analytical Data ........................ 14

$2.2 \quad$ Results......................................... 14

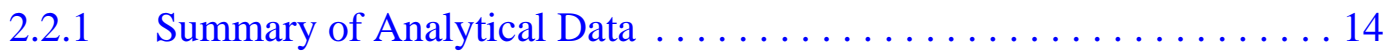

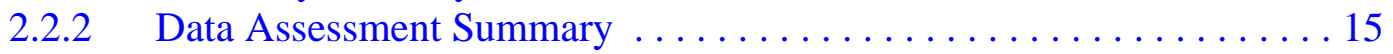

$2.3 \quad$ Justification for No Further Action. . . . . . . . . . . . . . . . . . 15

2.3.1 Final Action Levels........................... 21

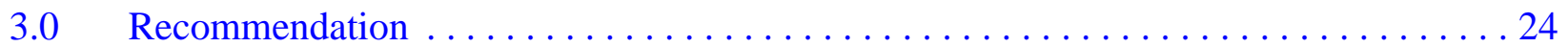

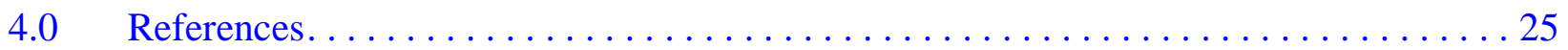

\section{Appendix A - Corrective Action Investigation Results}

A.1.0 Introduction. . . . . . . . . . . . . . . . . . . . . . . . . . . . . . . A-1

A.1.1 Project Objectives................................ A-2

A.1.2 Contents ..................................... A-3

A.2.0 Investigation Overview $\ldots \ldots \ldots \ldots \ldots \ldots \ldots \ldots \ldots \ldots \ldots \ldots \ldots \ldots \ldots \ldots \ldots \ldots \ldots$

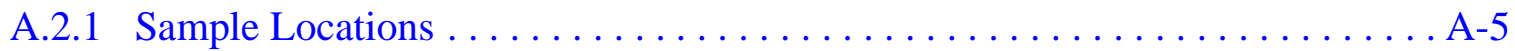

A.2.2 Investigation Activities . . . . . . . . . . . . . . . . . . . .

A.2.2.1 Radiological Surveys $\ldots \ldots \ldots \ldots \ldots \ldots \ldots \ldots \ldots \ldots \ldots$ A 6 . 6

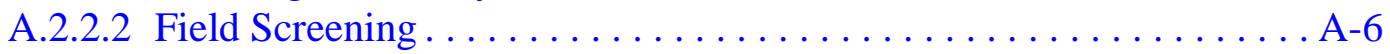

A.2.2.3 Surface and Subsurface Soil Sampling................ A-7

A.2.2.4 Waste Characterization and Potential Source Material Sampling .... A-7

A.2.3 Laboratory Analytical Information. . . . . . . . . . . . . . . . . . A -8

A.2.4 Comparison to Action Levels . . . . . . . . . . . . . . . . . . A-9

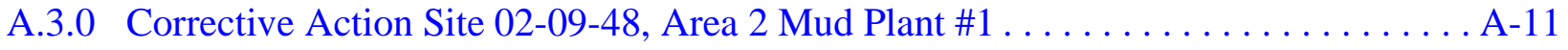

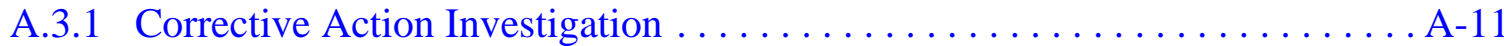

A.3.1.1 Field Screening ....................... A 12

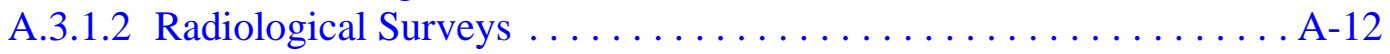

A.3.1.3 Visual Inspections........................... A

A.3.1.4 Sample Collection.......................... A-13

A.3.1.5 Deviations........................... A 13

A.3.2 Investigation Results. ........................... A-13

A.3.2.1 Volatile Organic Compounds ...................... A-15

A.3.2.2 Semivolatile Organic Compounds ................... A-15 


\section{Table of Contents (Continued)}

A.3.2.3 Total Petroleum Hydrocarbons. . . . . . . . . . . . . . . A-16

A.3.2.4 RCRA Metals . ............................ A-16

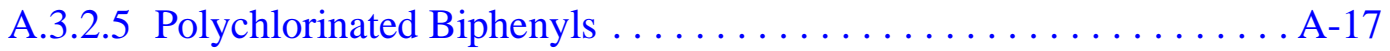

A.3.2.6 Gamma-Emitting Radionuclides . . . . . . . . . . . . . A-17

A.3.2.7 Plutonium, Strontium-90, and Uranium Isotopes . . . . . . . . . . . A-18

A.3.3 Nature and Extent of Contamination . . . . . . . . . . . . . A-18

A.3.4 Revised Conceptual Site Model . . . . . . . . . . . . . . . A-18

A.4.0 Corrective Action Site 03-09-02, Mud Dump Trenches. . . . . . . . . . . . . . . . . A-19

A.4.1 Corrective Action Investigation . . . . . . . . . . . . . . . A-19

A.4.1.1 Field Screening . . . . . . . . . . . . . . . . . . A-19

A.4.1.2 Radiological Surveys . . . . . . . . . . . . . . . . . . A-22

A.4.1.3 Visual Inspections. . . . . . . . . . . . . . . . . . A-22

A.4.1.4 Sample Collection........................... A-22

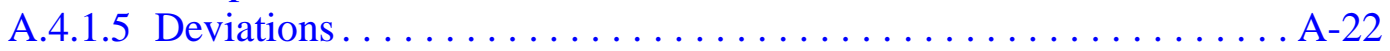

A.4.2 Investigation Results. . . . . . . . . . . . . . . . . . A-25

A.4.2.1 Volatile Organic Compounds . . . . . . . . . . . . . . . A-25

A.4.2.2 Semivolatile Organic Compounds . . . . . . . . . . . . . . . A-26

A.4.2.3 Total Petroleum Hydrocarbons. . . . . . . . . . . . . . . . A-26

A.4.2.4 RCRA Metals . . . . . . . . . . . . . . . . . . . A-27

A.4.2.5 Polychlorinated Biphenyls . . . . . . . . . . . . . . A-27

A.4.2.6 Gamma-Emitting Radionuclides . . . . . . . . . . . . . . A-27

A.4.2.7 Plutonium, Strontium-90, and Uranium Isotopes . . . . . . . . . . . A-31

A.4.3 Nature and Extent of Contamination . . . . . . . . . . . . . A-32

A.4.4 Revised Conceptual Site Model . . . . . . . . . . . . . . . . A-32

A.5.0 Corrective Action Site 12-09-01, Mud Pit . . . . . . . . . . . . . . . . . A-33

A.5.1 Corrective Action Investigation $\ldots \ldots \ldots \ldots \ldots \ldots \ldots \ldots \ldots \ldots \ldots \ldots \ldots \ldots \ldots \ldots$

A.5.1.1 Field Screening . . . . . . . . . . . . . . . . . A-34

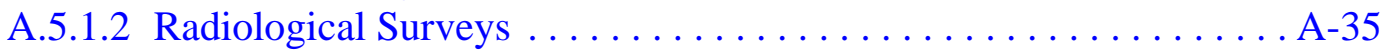

A.5.1.3 Visual Inspections. . . . . . . . . . . . . . . . A-35

A.5.1.4 Sample Collection. ....................... A-35

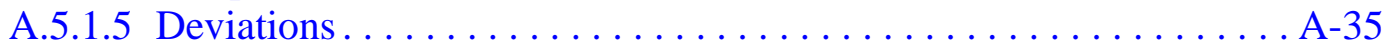

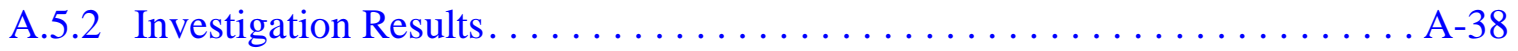

A.5.2.1 Volatile Organic Compounds . . . . . . . . . . . . . . . A-38

A.5.2.2 Semivolatile Organic Compounds . . . . . . . . . . . . . . . A-39

A.5.2.3 Total Petroleum Hydrocarbons . . . . . . . . . . . . . . . . A-39

A.5.2.4 RCRA Metals . . . . . . . . . . . . . . . . . . . A-39

A.5.2.5 Polychlorinated Biphenyls . . . . . . . . . . . . . . . A-39

A.5.2.6 Gamma-Emitting Radionuclides . . . . . . . . . . . . . A-41

A.5.2.7 Plutonium, Strontium-90, and Uranium Isotopes . . . . . . . . . . . A-42 


\section{Table of Contents (Continued)}

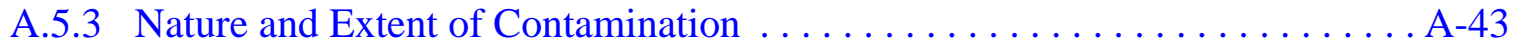

A.5.4 Revised Conceptual Site Model . . . . . . . . . . . . . . . . . . . . . A-43

A.6.0 Corrective Action Site 12-09-08, Mud Pit . . . . . . . . . . . . . . . . . . . . . . A-44

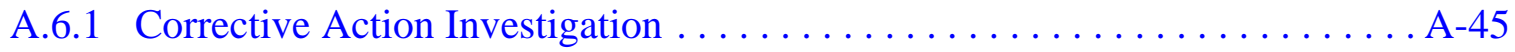

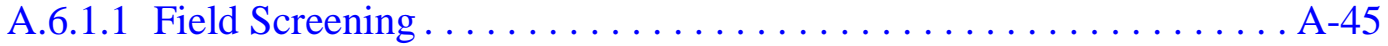

A.6.1.2 Radiological Surveys . . . . . . . . . . . . . . . A-46

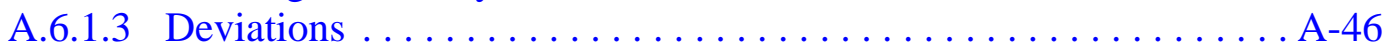

A.6.1.4 Visual Inspections. . . . . . . . . . . . . . . . . . A-46

A.6.1.5 Sample Collection. ......................... A-46

A.6.2 Investigation Results. . . . . . . . . . . . . . . . . . A

A.6.2.1 Volatile Organic Compounds . . . . . . . . . . . . . . . . . . . A-48

A.6.2.2 Semivolatile Organic Compounds . . . . . . . . . . . . . . . . . A-48

A.6.2.3 Total Petroleum Hydrocarbons. . . . . . . . . . . . . . . . . . . A-49

A.6.2.4 RCRA Metals . . . . . . . . . . . . . . . . . . . . . . . . . A A-49

A.6.2.5 Polychlorinated Biphenyls . . . . . . . . . . . . . . . . . A-49

A.6.2.6 Gamma-Emitting Radionuclides . . . . . . . . . . . . . . A-50

A.6.2.7 Plutonium, Strontium-90, and Uranium Isotopes . . . . . . . . . . . A-50

A.6.3 Nature and Extent of Contamination . . . . . . . . . . . . . . . A-52

A.6.4 Revised Conceptual Site Model . . . . . . . . . . . . . . . . A-52

A.7.0 Corrective Action Site $12-30-14$, Cellar . . . . . . . . . . . . . . . . . A-53

A.7.1 Corrective Action Investigation $\ldots \ldots \ldots \ldots \ldots \ldots \ldots \ldots \ldots \ldots \ldots \ldots \ldots \ldots \ldots \ldots$

A.7.1.1 Field Screening . . . . . . . . . . . . . . . . . . . A-54

A.7.1.2 Radiological Surveys . . . . . . . . . . . . . . . A -55

A.7.1.3 Visual Inspections. . . . . . . . . . . . . . . . . A-55

A.7.1.4 Sample Collection. ........................ A-55

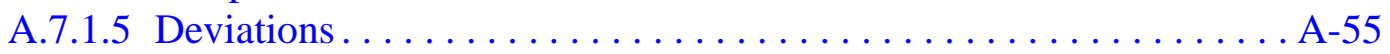

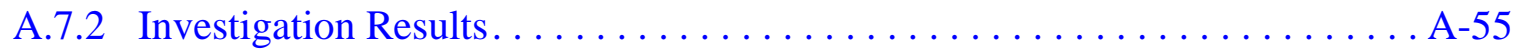

A.7.2.1 Volatile Organic Compounds . . . . . . . . . . . . . . . . . A-57

A.7.2.2 Semivolatile Organic Compounds . . . . . . . . . . . . . . . A-57

A.7.2.3 Total Petroleum Hydrocarbons . . . . . . . . . . . . . . . . . A-58

A.7.2.4 RCRA Metals . . . . . . . . . . . . . . . . . . . A A-59

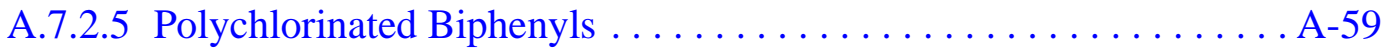

A.7.2.6 Gamma-Emitting Radionuclides . . . . . . . . . . . . . . . . A A-59

A.7.2.7 Plutonium, Strontium-90, and Uranium Isotopes . . . . . . . . . . . A-61

A.7.3 Nature and Extent of Contamination . . . . . . . . . . . . . . . A-61

A.7.4 Revised Conceptual Site Model . . . . . . . . . . . . . . . . . A-62

A.8.0 Waste Management. . . . . . . . . . . . . . . . . . . . . . . . A-63

A.8.1 Investigation-Derived Waste $\ldots \ldots \ldots \ldots \ldots \ldots \ldots \ldots \ldots \ldots \ldots \ldots \ldots \ldots$ 


\section{Table of Contents (Continued)}

A.8.2 IDW Waste Streams . . . . . . . . . . . . . . . . . . . . . . 63

A.8.3 Waste Characterization............................... A-64

A.8.4 Waste Disposal ................................... A-64

A.8.5 Potential Remediation Wastes $\ldots \ldots \ldots \ldots \ldots \ldots \ldots \ldots \ldots \ldots \ldots \ldots \ldots \ldots \ldots$

A.9.0 Quality Assurance. .............................. A-66

A.9.1 Data Validation. ............................... A-66

A.9.1.1 Tier I Evaluation . . . . . . . . . . . . . . . . . . . . A-66

A.9.1.2 Tier II Evaluation $\ldots \ldots \ldots \ldots \ldots \ldots \ldots \ldots \ldots \ldots \ldots \ldots \ldots \ldots \ldots \ldots$

A.9.1.3 Tier III Evaluation $\ldots \ldots \ldots \ldots \ldots \ldots \ldots \ldots \ldots \ldots \ldots \ldots \ldots \ldots \ldots$

A.9.2 Field Quality Control Samples $\ldots \ldots \ldots \ldots \ldots \ldots \ldots \ldots \ldots \ldots \ldots$ A-70

A.9.2.1 Laboratory Quality Control Samples . . . . . . . . . . . A A-70

A.9.3 Field Nonconformances . . . . . . . . . . . . . . . . . . . . . A-71

A.9.4 Laboratory Nonconformances $\ldots \ldots \ldots \ldots \ldots \ldots \ldots \ldots \ldots \ldots \ldots . \ldots . \ldots . \ldots \ldots$

A.10.0 Summary .................................

A.11.0 References................................... A-73

\section{Appendix B - Data Assessment}

B.1.0 Data Assessment...................................... B-1

B.1.1 Review DQOs and Sampling Design ....................... B-2

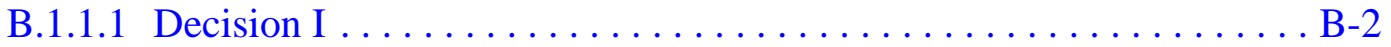

B.1.1.1.1 DQO Provisions To Limit

False Negative Decision Error $\ldots \ldots \ldots \ldots \ldots \ldots$ B-2

B.1.1.1.2 DQO Provisions To Limit

False Positive Decision Error . . . . . . . . . . . B-6

B.1.1.2 Decision II ............................. B-6

B.1.1.3 Sampling Design........................

B.1.2 Conduct a Preliminary Data Review $\ldots \ldots \ldots \ldots \ldots \ldots \ldots \ldots \ldots \ldots \ldots \ldots \ldots \ldots$

B.1.3 Select the Test and Identify Key Assumptions.................. B-7

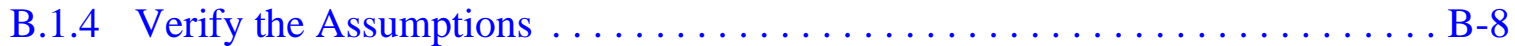

B.1.4.1 Other DQO Commitments ...................... B-8

B.1.5 Draw Conclusions from the Data ......................

B.1.5.1 Decision Rules for Decision I. . . . . . . . . . . . . . . . . B-9

B.1.5.2 Decision Rules for Decision II . . . . . . . . . . . . . . . B-9

B.2.0 References.................................. 


\section{Table of Contents (Continued)}

\section{Appendix C - Risk Assessment}

C.1.0 Risk Assessment . . . . . . . . . . . . . . . . . .

C.1.1 A. Scenario . . . . . . . . . . . . .

C.1.2 B. Site Assessment . . . . . . . . . . . . . . . . . . . . C-4

C.1.3 C. Site Classification and Initial Response Action . . . . . . . . . . . . . . C-4

C.1.4 D. Development of Tier I Lookup Table of Risk-Based Screening Levels . . . C-5

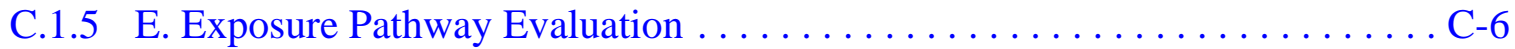

C.1.6 F. Comparison of Site Conditions with Tier I Risk-Based Screening Levels . C-6

C.1.7 G. Evaluation of Tier I Results . . . . . . . . . . . . . . . . . C-6

C.1.8 H. Tier I Remedial Action Evaluation . . . . . . . . . . . . . . C-6

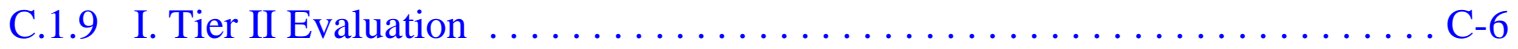

C.2.0 Recommendations........................... C-7

C.3.0 References.............................

Appendix D - Closure Activity Summary

D.1.0 Closure Activity Summary . . . . . . . . . . . . . . . . . . .

Attachment D-1 Load Verification Forms

Appendix E - Sample Location Coordinates

E.1.0 Sample Location Coordinates . . . . . . . . . . . . . . .

E.2.0 References............................... E-7

Appendix F - Nevada Division of Environmental Protection Comments 


\section{List of Figures}

Number

Title

Page

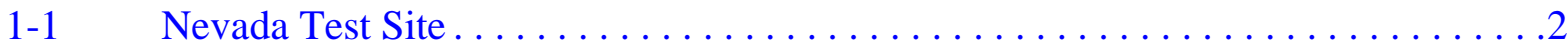

1-2 Corrective Action Unit 234, CAS Location Map ...................... 3

A.3-1 Corrective Action Site $02-09-48 \ldots \ldots \ldots \ldots \ldots \ldots \ldots \ldots \ldots \ldots \ldots \ldots \ldots . \ldots \ldots$

A.3-2 Sample Locations for CAS $02-09-48 \ldots \ldots \ldots \ldots \ldots \ldots \ldots \ldots \ldots$ A-14

A.4-1 Mud Trenches in Southern Footprint $\ldots \ldots \ldots \ldots \ldots \ldots \ldots \ldots \ldots$ A-20

A.4-2 Suction Pit in Northern Footprint $\ldots \ldots \ldots \ldots \ldots \ldots \ldots \ldots \ldots \ldots \ldots . . \ldots \ldots$

A.4-3 Radiological Survey for CAS $03-09-02 \ldots \ldots \ldots \ldots \ldots \ldots \ldots \ldots$ A-23

A.4-4 Sample Locations for CAS $03-09-02 \ldots \ldots \ldots \ldots \ldots \ldots \ldots \ldots \ldots$ A-24

A.5-1 Debris at CAS $12-09-01 \ldots \ldots \ldots \ldots \ldots \ldots \ldots \ldots \ldots \ldots \ldots \ldots \ldots \ldots \ldots \ldots \ldots . \ldots \ldots$

A.5-2 Flyover Radiation Readings $\ldots \ldots \ldots \ldots \ldots \ldots \ldots \ldots \ldots \ldots \ldots \ldots \ldots \ldots \ldots$

A.5-3 Sample Locations for CAS $12-09-01 \ldots \ldots \ldots \ldots \ldots \ldots \ldots \ldots$ A-37

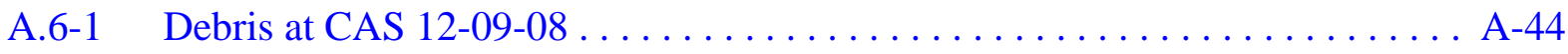

A.6-2 Sample Locations for CAS $12-09-08 \ldots \ldots \ldots \ldots \ldots \ldots \ldots \ldots \ldots$ A-47

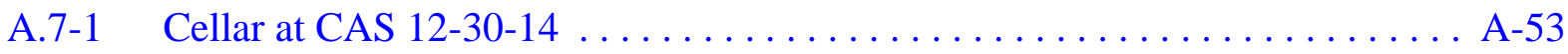

A.7-2 Sample Locations for CAS $12-30-14 \ldots \ldots \ldots \ldots \ldots \ldots \ldots \ldots \ldots$ A-56

C.1-1 Risk-Based Corrective Action Decision Process ...................

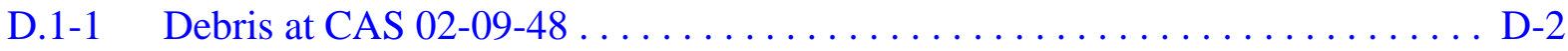

D.1-2 Area after Debris Removal at CAS $02-09-48 \ldots \ldots \ldots \ldots \ldots \ldots \ldots$ D-2

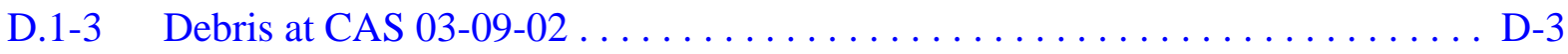

D.1-4 Area after Debris Removal at CAS $03-09-02 \ldots \ldots \ldots \ldots \ldots \ldots \ldots$ D-3

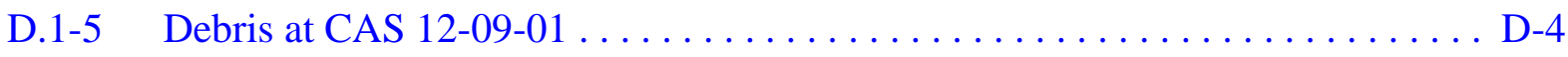

D.1-6 Area after Pipe Debris Removal at CAS $12-09-01 \ldots \ldots \ldots \ldots \ldots \ldots$ D-4 


\section{List of Figures (Continued)}

Number

Title

Page

D.1-7 Area after Cylinder Debris Removal at CAS 12-09-01 . . . . . . . . . . D-5

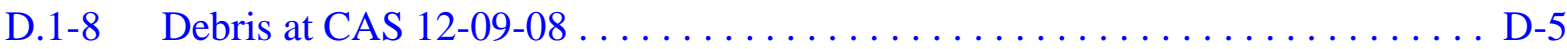

D.1-9 Area after Pipe and Drum Debris Removal at CAS 12-09-08 . . . . . . . . D-6

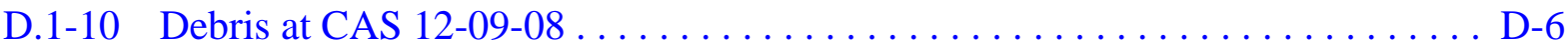

D.1-11 Area after Drum Debris Removal at CAS 12-09-08. . . . . . . . . . . . D-7

E.1-1 Sample Location Coordinates for CAS $02-09-48 \ldots \ldots \ldots \ldots \ldots \ldots \ldots \ldots$. . . . .

E.1-2 Sample Location Coordinates for CAS $03-09-02 \ldots \ldots \ldots \ldots \ldots \ldots \ldots \ldots$

E.1-3 Sample Location Coordinates for CAS-12-09-01 . . . . . . . . . . . . E-4

E.1-4 Sample Locations Coordinates for CAS $12-09-08 \ldots \ldots \ldots \ldots \ldots \ldots \ldots \ldots$

E.1-5 Sample Location Coordinates for CAS $12-30-14 \ldots \ldots \ldots \ldots \ldots \ldots \ldots \ldots$ 


\section{List of Tables}

Number

Title

Page

2-1 Maximum Concentration of Detected Contaminants in Soil at

CAS 02-09-48, Area 2 Mud Plant $\# 1 \ldots \ldots \ldots \ldots \ldots \ldots \ldots \ldots \ldots \ldots \ldots \ldots \ldots \ldots$

2-2 Maximum Concentration of Detected Contaminants in Soil at

CAS 03-09-02, Mud Dump Trenches..................... 17

2-3 Maximum Concentration of Detected Contaminants in Soil at

CAS 12-09-01, Mud Pit . . . . . . . . . . . . . . . . . . . . . 18

2-4 Maximum Concentration of Detected Contaminants in Soil at

CAS 12-09-08, Mud Pit . . . . . . . . . . . . . . . . . . . . . . . . 19

2-5 Maximum Concentration of Detected Sediment Contaminants for

CAS 12-30-14, Cellar............................... 20

2-6 Maximum Concentration of Detected Liquid Contaminants for

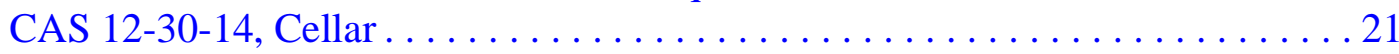

2-7 Definition of Final Action Levels for CAU 234

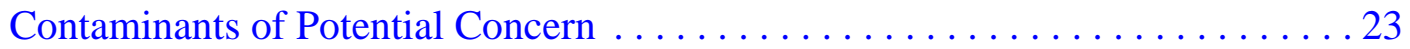

A.2-1 Corrective Action Investigation Activities Conducted at Each

Corrective Action Site To Meet Corrective Action Investigation Plan

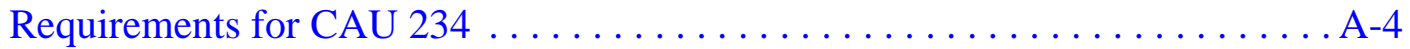

A.2-2 Laboratory Analytical Parameters and Methods,

CAU 234 Investigation Samples. . . . . . . . . . . . . . . . . . . A-8

A.3-1 Samples Collected at CAS 02-09-48, Area 2 Mud Plant \#1............. A-12

A.3-2 Soil Sample Results of SVOCs Detected above Minimum Detectable

Concentrations at CAS 02-09-48, Area 2 Mud Plant \#1 . . . . . . . . . . A-15

A.3-3 Soil Sample Results for RCRA Metals Detected above Minimum Detectable Concentrations at CAS 02-09-48, Area 2 Mud Plant \#1 . . . . . . . . . A-16 


\section{List of Tables (Continued)}

Number

Title

Page

A.3-4 Soil Sample Results for Gamma-Emitting Radionuclides Detected above Minimum Detectable Concentrations at CAS 02-09-48, Area 2 Mud Plant $\# 1$. . . . . . . . . . . . . . . . . . . . . . . . . A-17

A.3-5 Soil Sample Results for Isotopes Detected above Minimum Detectable Concentrations at CAS 02-09-48, Area 2 Mud Plant \#1 . . . . . . . . . . A-18

A.4-1 Samples Collected at CAS 03-09-02, Mud Dump Trenches . . . . . . . . . . . A-21

A.4-2 Soil Sample Results for VOCs Detected above Minimum Detectable Concentrations at CAS 03-09-02, Mud Dump Trenches. . . . . . . . . . . . . A-25

A.4-3 Soil Sample Results for SVOCs Detected above Minimum Detectable Concentrations at CAS 03-09-02, Mud Dump Trenches. . . . . . . . . . . A-26

A.4-4 Soil Sample Results for TPH-DRO Detected above Minimum Detectable Concentrations at CAS 03-09-02, Mud Dump Trenches. . . . . . . . . . . A-27

A.4-5 Soil Sample Results for RCRA Metals Detected above Minimum Detectable Concentrations at CAS 03-09-02, Mud Dump Trenches. . . . . . . . . . . . . A-28

A.4-6 Soil Sample Results for Gamma-Emitting Radionuclides Detected above Minimum Detectable Concentrations at CAS 03-09-02, Mud Dump Trenches . . . . . . . . . . . . . . . . . . . . . . . A A-29

A.4-7 Soil Sample Results for Isotopes Detected above Minimum Detectable Concentrations at CAS 03-09-02, Mud Dump Trenches. . . . . . . . . . . A-31

A.5-1 Samples Collected at CAS 12-09-01, Mud Pit . . . . . . . . . . . . A-34

A.5-2 Soil Sample Results for VOCs Detected above Minimum Detectable Concentrations at CAS 12-09-01, Mud Pit ............... A-38

A.5-3 Soil Sample Results for SVOCs Detected above Minimum Detectable Concentrations at CAS 12-09-01, Mud Pit . . . . . . . . . . . . A-39

A.5-4 Soil Sample Results for TPH-DRO Detected above Minimum Detectable Concentrations at CAS 12-09-01, Mud Pit . . . . . . . . . . . . . A-40 


\section{List of Tables (Continued)}

Number

Title

Page

A.5-5 Soil Sample Results for RCRA Metals Detected above Minimum Detectable Concentrations at CAS 12-09-01, Mud Pit . . . . . . . . . . . . . A-40

A.5-6 Soil Sample Results for Gamma-Emitting Radionuclides Detected above Minimum Detectable Concentrations at CAS 12-09-01, Mud Pit . . . . A-41

A.5-7 Soil Sample Results for Isotopes Detected above Minimum Detectable Concentrations at CAS 12-09-01, Mud Pit . . . . . . . . . . . . . A-42

A.6-1 Samples Collected at CAS 12-09-08, Mud Pit . . . . . . . . . . . . . A-45

A.6-2 Soil Sample Results for SVOCs Detected above Minimum Detectable Concentrations at CAS 12-09-08, Mud Pit . . . . . . . . . . . . . . A-48

A.6-3 Soil Sample Results for TPH-DRO Detected above Minimum Detectable Concentrations at CAS 12-09-08, Mud Pit . . . . . . . . . . . . . . . . A-49

A.6-4 Soil Sample Results for RCRA Metals Detected above Minimum Detectable Concentrations at CAS 12-09-08, Mud Pit . . . . . . . . . . . . . . . A A-50

A.6-5 Soil Sample Results for Gamma-Emitting Radionuclides Detected above Minimum Detectable Concentrations at CAS 12-09-08, Mud Pit . . . . A A-51

A.6-6 Soil Sample Results for Isotopes Detected above Minimum Detectable Concentrations at CAS 12-09-08, Mud Pit . . . . . . . . . . . . A-51

A.7-1 Samples Collected at CAS 12-30-14, Cellar . . . . . . . . . . . . . . A-54

A.7-2 Sediment PSM Sample Results for VOCs Detected above Minimum Detectable Concentrations at CAS 12-30-14, Cellar . . . . . . A-57

A.7-3 Liquid PSM Sample Results for VOCs Detected above Minimum Detectable Concentrations at CAS 12-30-14, Cellar . . . . . . A-58

A.7-4 Sediment PSM Sample Results for SVOCs Detected above Minimum Detectable Concentrations at CAS 12-30-14, Cellar . . . . . . A-58 


\section{List of Tables (Continued)}

Number

Title

Page

A.7-5 Sediment PSM Sample Results for TPH-DRO Detected above Minimum Detectable Concentrations at CAS 12-30-14, Cellar . . . . . . A-59

A.7-6 Sediment PSM Sample Results for RCRA Metals Detected above Minimum Detectable Concentrations at CAS 12-30-14, Cellar . . . . . . A-60

A.7-7 Liquid PSM Sample Results for RCRA Metals Detected above Minimum Detectable Concentrations at CAS 12-30-14, Cellar . . . . . . A-60

A.7-8 Sediment PSM Sample Results for Gamma-Emitting Radionuclides Detected above Minimum Detectable Concentrations at 12-30-14, Cellar . . . . . . . . . A-61

A.7-9 Sediment PSM Sample Results for Isotopes Detected above Minimum Detectable Concentrations at CAS 12-30-14, Cellar . . . . . . A-62

A.8-1 CAU 234 Projected Waste Inventory and Preliminary Disposal Recommendation Summary. . . . . . . . . . . . . . . . A-65

B.1-1 CAU 234 Analyses Performed $\ldots \ldots \ldots \ldots \ldots \ldots \ldots \ldots \ldots \ldots \ldots$

B.1-2 Precision Qualifications for CAU $234 \ldots \ldots \ldots \ldots \ldots \ldots \ldots \ldots \ldots \ldots$

B.1-3 Accuracy Measurements for CAU $234 \ldots \ldots \ldots \ldots \ldots \ldots \ldots \ldots \ldots$

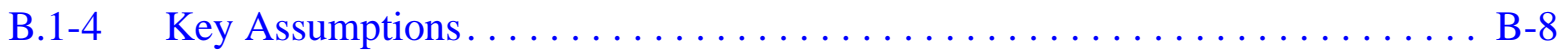




\section{List of Acronyms and Abbreviations}

ASTM American Society for Testing and Materials

bgs $\quad$ Below ground surface

BMP Best management practice

CADD Corrective Action Decision Document

CAI Corrective Action Investigation

CAIP Corrective Action Investigation Plan

CAS Corrective Action Site

CAU Corrective Action Unit

CLP Contract Laboratory Program

$\mathrm{cm} \quad$ Centimeter

COC Contaminant of concern

COPC Contaminant of potential concern

CPS Counts per second

CR Closure Report

CSM Conceptual site model

DOE U.S. Department of Energy

DQA Data quality assessment

DQI Data quality indicator

DQO Data quality objective

DRO Diesel-range organics

EML Environmental Measurements Laboratory

EPA U.S. Environmental Protection Agency

FAL Final action level

FD Field duplicate

FFACO Federal Facility Agreement and Consent Order

FSL Field-screening level 


\section{List of Acronyms and Abbreviations (Continued)}

\begin{tabular}{|c|c|}
\hline FSR & Field-screening result \\
\hline $\mathrm{ft}$ & Foot \\
\hline $\mathrm{ft}^{3}$ & Cubic foot \\
\hline gal & Gallon \\
\hline GPS & Global Positioning System \\
\hline HASL & Health and Safety Laboratory \\
\hline ID & Identification \\
\hline IDW & Investigation-derived waste \\
\hline LCS & Laboratory control sample \\
\hline MB & Method blank \\
\hline MDC & Minimum detectable concentration \\
\hline $\mathrm{mg} / \mathrm{kg}$ & Milligrams per kilogram \\
\hline $\mathrm{mg} / \mathrm{L}$ & Milligrams per liter \\
\hline $\mathrm{mrem} / \mathrm{yr}$ & Millirem per year \\
\hline MS & Matrix spike \\
\hline MSD & Matrix spike duplicate \\
\hline N/A & Not applicable \\
\hline NAC & Nevada Administrative Code \\
\hline NAD & North American Datum \\
\hline NCRP & National Council on Radiation Protection and Measurements \\
\hline NDEP & Nevada Division of Environmental Protection \\
\hline $\mathrm{NIOSH}$ & National Institute for Occupational Safety and Health \\
\hline NIST & National Institute of Standards and Technology \\
\hline NNSA/NSO & $\begin{array}{l}\text { U.S. Department of Energy, National Nuclear Security Administration } \\
\text { Nevada Site Office }\end{array}$ \\
\hline NTS & Nevada Test Site \\
\hline NV/YMP & Nevada/Yucca Mountain Project \\
\hline
\end{tabular}




\section{List of Acronyms and Abbreviations (Continued)}

\begin{tabular}{|c|c|}
\hline PAL & Preliminary action level \\
\hline PB & Preparation blank \\
\hline PCB & Polychlorinated biphenyl \\
\hline $\mathrm{pCi} / \mathrm{g}$ & Picocuries per gram \\
\hline $\mathrm{pCi} / \mathrm{L}$ & Picocuries per liter \\
\hline POC & Performance objective criteria \\
\hline PPE & Personal protective equipment \\
\hline PRG & Preliminary Remediation Goal \\
\hline PSM & Potential source material \\
\hline PVC & Polyvinyl chloride \\
\hline $\mathrm{Pu}$ & Plutonium \\
\hline QA & Quality assurance \\
\hline QAPP & Quality Assurance Project Plan \\
\hline QC & Quality control \\
\hline RBCA & Risk-based corrective action \\
\hline RBSL & Risk-based screening level \\
\hline RCRA & Resource Conservation and Recovery Act \\
\hline RPD & Relative percent difference \\
\hline SCL & Sample collection log \\
\hline SDG & Sample delivery group \\
\hline SNJV & Stoller-Navarro Joint Venture \\
\hline SOP & Standard operating procedure \\
\hline $\mathrm{Sr}$ & Strontium \\
\hline SSTL & Site-specific target level \\
\hline SVOC & Semivolatile organic compound \\
\hline TC & Toxicity characteristic \\
\hline
\end{tabular}




\section{List of Acronyms and Abbreviations (Continued)}

TCLP Toxicity characteristic leaching procedure

TPH Total petroleum hydrocarbons

U Uranium

UST Underground storage tank

UTM Universal Transverse Mercator

VOC Volatile organic compound

WM Waste management

$\% \mathrm{R} \quad$ Percent recovery

$\mu \mathrm{R} / \mathrm{hr} \quad$ Microroentgens per hour 


\section{Executive Summary}

This Corrective Action Decision Document/Closure Report has been prepared for Corrective Action Unit (CAU) 234, Mud Pits, Cellars, and Mud Spills, located in Areas 2, 3, 4, 12, and 15 at the Nevada Test Site, Nevada, in accordance with the Federal Facility Agreement and Consent Order (FFACO, 1996; as amended February 2008). Corrective Action Unit 234 is comprised of the following 12 corrective action sites:

- 02-09-48, Area 2 Mud Plant \#1

- 02-09-49, Area 2 Mud Plant \#2

- 02-99-05, Mud Spill

- 03-09-02, Mud Dump Trenches

- 04-44-02, Mud Spill

- 04-99-02, Mud Spill

- 12-09-01, Mud Pit

- $12-09-04$, Mud Pit

- 12-09-08, Mud Pit

- 12-30-14, Cellar

- 12-99-07, Mud Dump

- 15-09-01, Mud Pit

The purpose of this Corrective Action Decision Document/Closure Report is to provide justification and documentation supporting the recommendation for closure of CAU 234 with no further corrective action. To achieve this, corrective action investigation (CAI) activities were performed as set forth in the Corrective Action Investigation Plan for Corrective Action Unit 234: Mud Pits, Cellars, and Mud Spills (NNSA/NSO, 2007). The purpose of the CAI was to fulfill the following data needs as defined during the data quality objective (DQO) process:

- Determine whether contaminants of concern are present.

- If contaminants of concern are present, determine their extent.

- Provide sufficient information and data to complete appropriate corrective actions.

The CAU 234 dataset from the investigation results was evaluated based on the data quality indicator parameters. This evaluation demonstrated the quality and acceptability of the dataset for use in fulfilling the DQO data needs. 
Analytes detected during the CAI were evaluated against final action levels (FALs) established in this document. The FAL for total petroleum hydrocarbons-diesel-range organics was established as the U.S. Environmental Protection Agency Region 9 Preliminary Remediation Goal values for the individual hazardous constituents of diesel. No CAU 234 samples contained contaminants that exceeded their respective FALs. Therefore, the DQO data needs were met, and it was determined that no corrective action (based on risk to human receptors) is necessary for the site.

Therefore, the U.S. Department of Energy, National Nuclear Security Administration Nevada Site Office provides the following recommendations:

- No further corrective action is needed for CAU 234 corrective action sites.

- No Corrective Action Plan is necessary.

- A Notice of Completion to the U.S. Department of Energy, National Nuclear Security Administration Nevada Site Office is requested from the Nevada Division of Environmental Protection for closure of CAU 234.

- Corrective Action Unit 234 should be moved from Appendix III to Appendix IV of the Federal Facility Agreement and Consent Order. 


\subsection{Introduction}

This Corrective Action Decision Document (CADD)/Closure Report (CR) presents information supporting closure of Corrective Action Unit (CAU) 234, Mud Pits, Cellars, and Mud Spills, Nevada Test Site (NTS), Nevada. The corrective actions proposed in this document are in accordance with the Federal Facility Agreement and Consent Order (FFACO) that was agreed to by the State of Nevada; U.S. Department of Energy (DOE), Environmental Management; U.S. Department of Defense; and DOE, Legacy Management (FFACO, 1996; as amended February 2008). The NTS is approximately 65 miles northwest of Las Vegas, Nevada (Figure 1-1).

Corrective Action Unit 234 is comprised of the following 12 corrective action sites (CASs) that are shown on Figure 1-2:

- 02-09-48, Area 2 Mud Plant \#1

- 02-09-49, Area 2 Mud Plant \#2

- 02-99-05, Mud Spill

- 03-09-02, Mud Dump Trenches

- 04-44-02, Mud Spill

- 04-99-02, Mud Spill

- 12-09-01, Mud Pit

- 12-09-04, Mud Pit

- 12-09-08, Mud Pit,

- 12-30-14, Cellar

- 12-99-07, Mud Dump

- 15-09-01, Mud Pit

A detailed discussion of the history of this CAU is presented in the Corrective Action Investigation Plan (CAIP) for Corrective Action Unit 234: Mud Pits, Cellars, and Mud Spills (NNSA/NSO, 2007). This document provides or references the specific information necessary to support closure of this CAU.

\subsection{Purpose}

This CADD/CR provides justification why no further corrective action is necessary. This justification is based on the activities that were conducted in accordance with the CAIP (NNSA/NSO, 2007). 


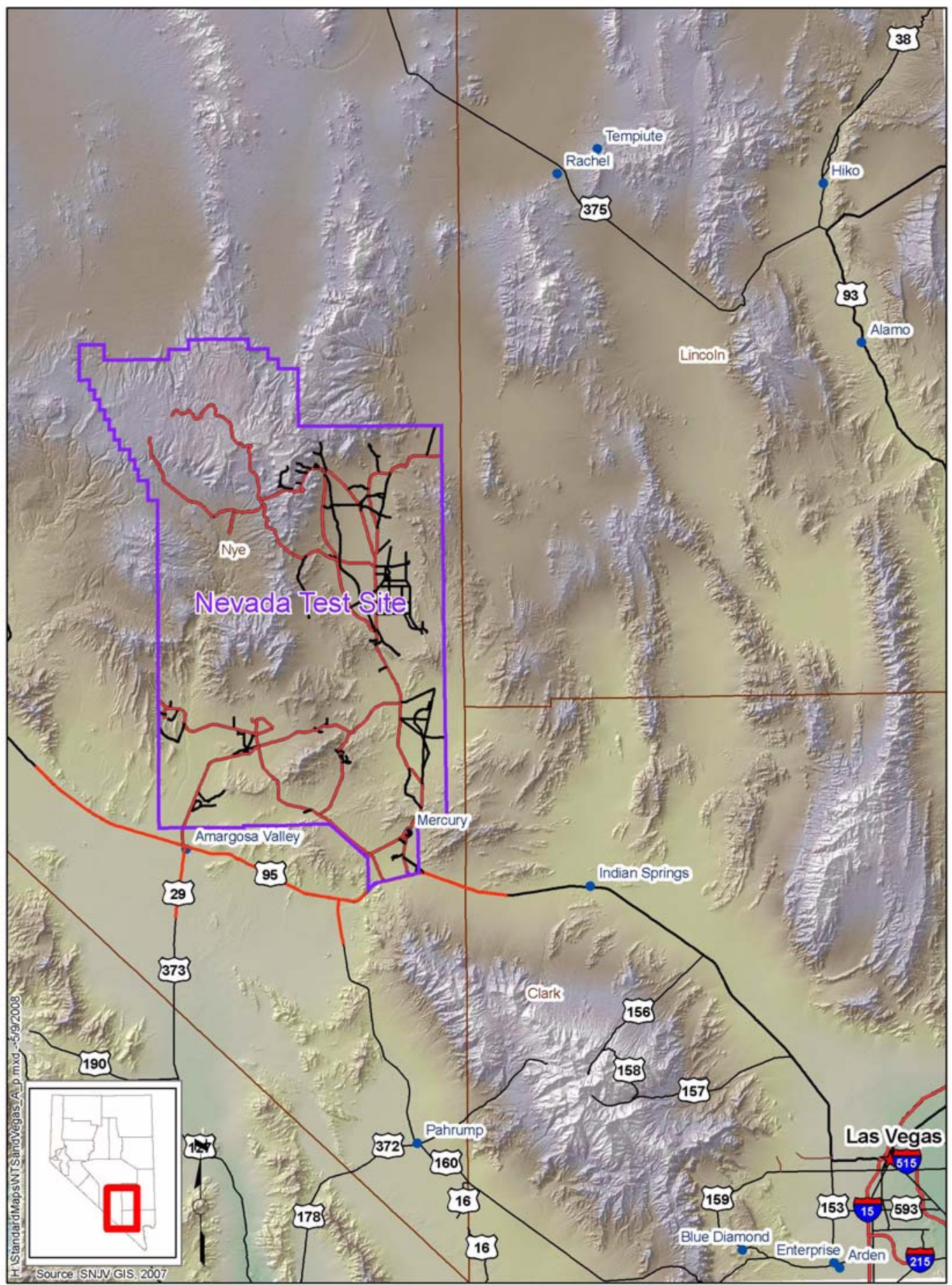

Figure 1-1

Nevada Test Site 


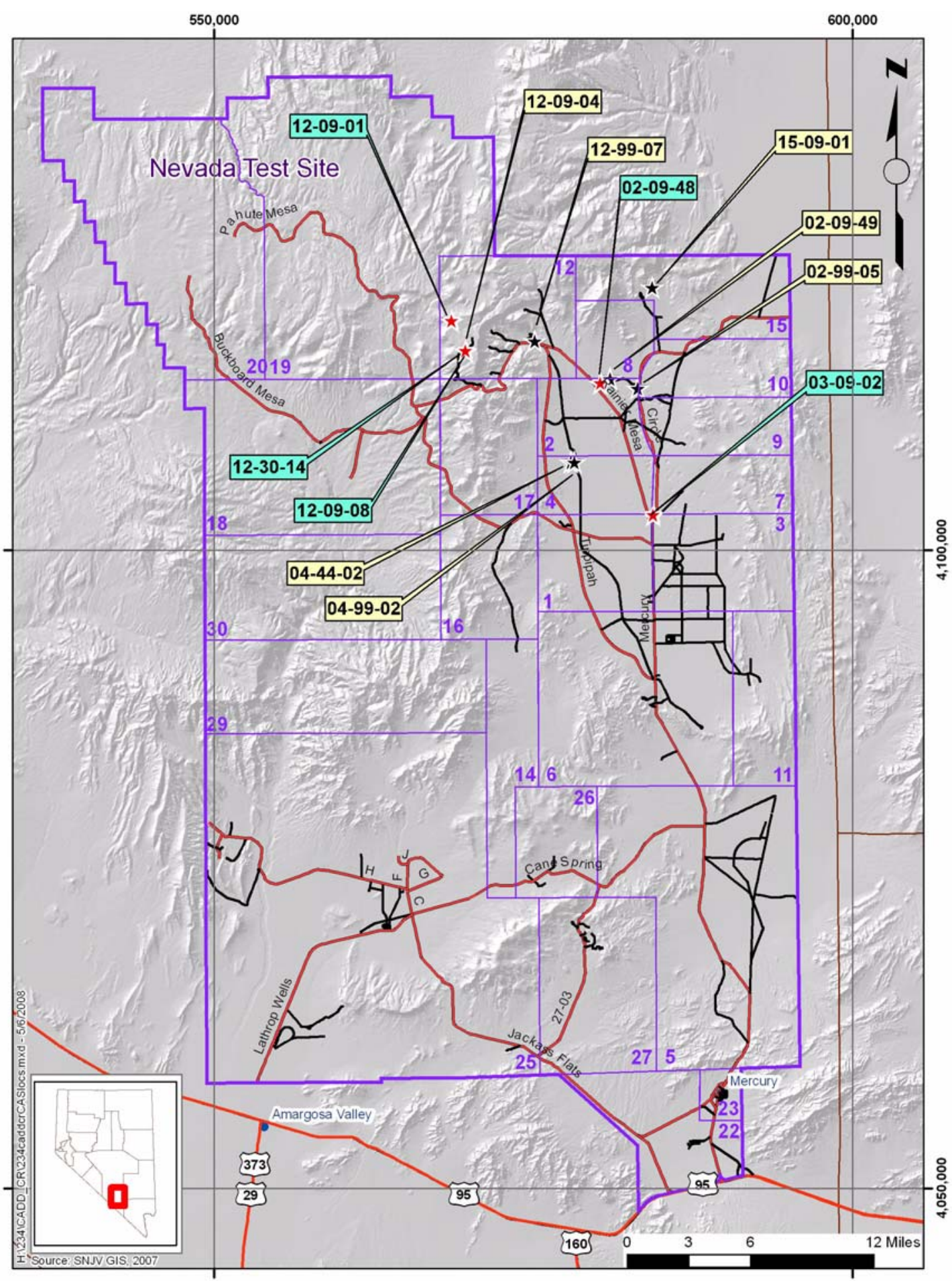

Figure 1-2

Corrective Action Unit 234, CAS Location Map 
Corrective Action Unit 234, Mud Pits, Cellars, and Mud Spills, consists of 12 inactive sites located in the northwestern portion of Area 2; the northwestern corner of Area 3; the northwestern portion of Area 4; the south-central, southwestern, and western portions of Area 12; and the southeastern portion of the panhandle on the northwest corner of Area 15. The 12 CAU 234 sites consist of mud pits (suction, reserve, and return); mud dumps; mud spills; concrete dumps and spills; a cellar; and articles of debris not specifically associated with a mud pit or spill.

The CAU 234 CAIP describes the criteria by which seven of the 12 CASs were determined to have sufficient information to support a no further action closure (NNSA/NSO, 2007). Therefore, additional information was not collected (or reported in this CADD/CR) for the following CASs:

- 02-09-49, Area 2 Mud Plant \#2

- 02-99-05, Mud Spill

- 04-44-02, Mud Spill

- 04-99-02, Mud Spill

- 12-09-04, Mud Pit

- 12-99-07, Mud Dump

- 15-09-01, Mud Pit

The remaining five CASs (02-09-48, 03-09-02, 12-09-01, 12-09-08, and 12-30-14; identified in green in Figure 1-2) contained debris or are associated with process knowledge that indicates potential presence of contaminants of potential concern (COPCs) not commonly associated with mud pits.

\subsection{Scope}

The scope of this CADD/CR is to justify that no further corrective action is required at CAU 234, Mud Pits, Cellars, and Mud Spills. The activities conducted to accomplish this scope included the following:

- Removal and disposal of surface debris and/or materials to facilitate sampling or as a best management practice (BMP)

- Radiological surveys

- $\quad$ Field screening

- Collection of environmental samples for laboratory analysis 
- Collection of source material samples to determine the potential to generate contaminants of concern (COCs) if released to the environment

- Collection of waste samples to determine the proper disposal of wastes

- Collection of quality control (QC) samples

\subsection{Corrective Action Decision Document/Closure Report Contents}

This CADD/CR is divided into the following sections and appendices:

Section 1.0 - Introduction: Summarizes the purpose, scope, and contents of this CADD/CR.

Section 2.0 - Corrective Action Investigation (CAI) Summary: Summarizes the investigation field activities, the results of the investigation, the need for corrective action, and a summary of the results of the data quality objective (DQO) assessment.

Section 3.0 - Recommendation: States why no further corrective action is required.

Section 4.0 - References: Provides a list of all referenced documents used in the preparation of this $\mathrm{CADD} / \mathrm{CR}$.

Appendix A - Corrective Action Investigation Results: Provides a description of the project objectives, field investigation and sampling activities, investigation results, waste management (WM), and quality assurance (QA).

Appendix B - Data Assessment: Provides a data quality assessment (DQA) that reconciles DQO assumptions and requirements to the investigation results.

Appendix C - Risk Assessment: Presents an evaluation of risk associated with the establishment of final action levels (FALs).

Appendix D - Closure Activity Summary: Provides details on the completed closure activities and supporting documentation.

Appendix E - Sample Location Coordinates: Provides the global positioning system (GPS) coordinates of sample locations for each CAS sampled during the CAI. 
Appendix F - Nevada Division of Environmental Protection (NDEP) Comments: Contains an NDEP letter stating that there were no comments on the draft version of this document.

\subsubsection{Applicable Programmatic Plans and Documents}

All investigation activities were performed in accordance with the following documents:

- CAIP for CAU 234, Mud Pits, Cellars, and Mud Spills (NNSA/NSO, 2007)

- Industrial Sites Quality Assurance Project Plan (QAPP) (NNSA/NV, 2002)

- FFACO (1996, as amended February 2008)

- Approved procedures

\subsubsection{Data Quality Assessment Summary}

The DQA is presented in Appendix B and includes an evaluation of the data quality indicators (DQIs) to determine the degree of acceptability and usability of the reported data in the decision-making process. The DQO process ensures that the right type, quality, and quantity of data will be available to support the resolution of those decisions at an appropriate level of confidence. Using both the DQO and DQA processes helps to ensure that DQO decisions are sound and defensible.

The DQA process as presented in Appendix B is comprised of the following steps:

- Step 1: Review DQOs and Sampling Design.

- Step 2: Conduct a Preliminary Data Review.

- Step 3: Select the Test.

- Step 4: Verify the Assumptions.

- Step 5: Draw Conclusions from the Data.

Sample locations that support the DQO decisions at each CAS are shown in Appendix A. Based on the results of the DQA presented in Appendix B, the information generated during the investigation supports the conceptual site model (CSM) assumptions, and the data collected met the DQOs and support their intended use in the decision-making process. 


\subsection{Corrective Action Investigation Summary}

Page 7 of 26

The following sections summarize the investigation activities and investigation results, and justify why no further corrective action is needed at CAU 234. Detailed investigation activities and results for individual CAU 234 CASs are presented in Appendix A.

\section{$2.1 \quad$ Investigation Activities}

Corrective action investigation activities were performed as set forth in the CAU 234 CAIP (NNSA/NSO, 2007) from October 29 through November 7, 2007. Additional sampling was conducted on January 23, 2008. The purpose of the CAU 234 CAI was to address the decision statements in the project-specific DQOs by:

- $\quad$ Determining whether COCs are present in the soils associated with CAU 234.

- Determining the lateral and vertical extent of identified COCs.

- Ensuring adequate data have been collected to close the sites under NDEP, Resource Conservation and Recovery Act (RCRA) (CFR, 2006a), Toxic Substances Control Act (CFR, 2006b), and DOE requirements.

The scope of the CAI included the following activities:

- Performing radiological surveys (i.e., static, scanning, and swipe collection).

- Field screening soil samples for total alpha and beta/gamma radiation.

- Collecting environmental samples for laboratory analyses to determine the presence of COCs and to define the vertical and lateral extent of COCs, if present.

- Collecting QC samples for laboratory analyses to ensure that the data generated from the analysis of investigation samples meet the requirements of the DQIs.

- Collecting liquid and solid material samples from the cellar system components at CAS 12-30-14 to identify whether the material contained in this structure is a potential source of environmental contamination.

Judgmental sampling schemes were implemented to select sample locations and evaluate analytical results, as outlined in the CAIP (NNSA/NSO, 2007). Judgmental sampling allows the methodical 
selection of sample locations that target the populations of interest (defined in the DQOs) rather than non-selective random locations.

For the judgmental sampling scheme, individual sample results (rather than average concentrations) are used to compare FALs. Therefore, statistical methods to generate site characteristics (averages) are not necessary. If good prior information is available on the target site of interest, then the sampling may be designed to collect samples only from areas known to have the highest concentration levels on the target site. If the observed concentrations from these samples are below the action level, then a decision can be made that the site contains safe levels of the contaminant without the samples being truly representative of the entire area (EPA, 2006).

The judgmental sampling design was used to confirm the existence of contamination at specific locations and provide information (such as extent of contamination) about specific areas of the site.

Confidence in judgmental sampling scheme decisions was established qualitatively by validation of the CSM and justification that sampling locations are the most likely locations to contain a COC, if a COC exists.

Waste characterization activities were conducted to gather sufficient information and data to support waste disposal decisions. Information regarding waste characterization is presented in Appendix A.

The following sections describe specific investigation activities conducted at each CAS. Additional information regarding the investigation is presented in Appendix A.

\subsubsection{Area 2 Mud Plant \#1 (CAS 02-09-48)}

The following subsections summarize the activities conducted at CAS 02-09-48.

\subsubsection{Radiological Survey}

As presented in the CAIP (NNSA/NSO, 2007), a radiological walkover survey was conducted across the drilling mud sump on May 23, 2006. The survey results were not distinguishable from background. As a result, no additional biased sample locations were identified. 


\subsubsection{Visual Inspection}

Visual inspections were conducted of the concrete sump and associated piping, and of the 55-gallon (gal) drum resting atop the drilling mud within the sump. No additional biased samples were identified.

\subsubsection{Field Screening}

The field-screening results (FSRs) were compared to field-screening levels (FSLs) to guide subsequent sampling decisions. No samples exceeded the FSLs established for the CAS. As a result, no additional samples were collected.

\subsubsection{Sample Collection}

Decision I sampling activities included the collection of five environmental soil samples (including one field duplicate [FD]) from the unused drilling mud within the sump. The sample identification (ID) numbers, locations, types, and analyses are listed in Table A.3-1. The sample locations are shown on Figure A.3-2. Samples were collected using grab sampling. Samples collected from this CAS are numbered 234A001 through 234A005.

\subsubsection{Conceptual Site Model Validation}

The CSM and associated discussion for this CAS are provided in the CAIP (NNSA/NSO, 2007). The information supporting the lack of contamination gathered during the CAI was consistent with the CSM, and all information gathered during the CAI supports and validates the CSM as presented in the CAIP (NNSA/NSO, 2007).

\subsubsection{Mud Dump Trenches (CAS 03-09-02)}

The following subsections summarize the activities conducted at CAS 03-09-02.

\subsubsection{Radiological Survey}

A radiological survey was conducted on October 25, 2007. Results of the radiological survey are presented as Figure A.4-3 and were not distinguishable from background readings. As a result, no additional samples were collected. 


\subsubsection{Visual Inspection}

Page 10 of 26

Visual inspections were made of the layout of the mud pits, their accessibility, and any other debris that would require investigation during the sampling effort. A length of blue pipe was identified under the tumbleweeds in the northern suction pit and was sampled at both ends. Otherwise, no additional sampling locations were identified.

\subsubsection{Field Screening}

Soil samples were screened in the field for alpha and beta/gamma radioactivity. The radiological FSRs were compared to FSLs to guide subsequent sampling decisions. The radiological FSRs were all below FSLs. As a result, no additional samples were collected.

\subsubsection{Sample Collection}

A total of 14 environmental soil characterization samples (including one FD and one matrix spike $[\mathrm{MS}] /$ matrix spike duplicate [MSD]) were collected from seven locations during investigation activities at CAS 03-09-02. The sample identification numbers, locations, types, and analyses are listed in Table A.4-1. The sample locations are shown in Figure A.4-4. Samples were collected using scoops and a hand auger. Samples collected at this CAS are numbered 234B001 through 234B014. A rinsate sample (234B501) was also collected and analyzed for all parameters plus gross alpha/beta and tritium.

Decision I surface and subsurface samples were collected from the lowest point of elevation within each of the mud pit trenches as identified through earlier photographs taken after rainstorms. The location of puddling of the rainwater indicated the low spots. No Decision II sampling was necessary as all Decision I sample results were below FALs.

\subsubsection{Conceptual Site Model Validation}

The CSM and associated discussion for this CAS are provided in the CAIP (NNSA/NSO, 2007). The information supporting the lack of contamination gathered during the CAI was consistent with the CSM, and all information gathered during the CAI supports and validates the CSM as presented in the CAIP (NNSA/NSO, 2007). 


\subsubsection{Mud Pit (CAS 12-09-01)}

The following subsections summarize the activities conducted at CAS 12-09-01.

\subsubsection{Radiological Survey}

An aerial radiological survey was conducted in 1994 of Area 12, including CAS 12-09-01. The results of the survey were not distinguishable from background. As a result, no additional samples were collected (see Figure A.5-3).

\subsubsection{Visual Inspection}

A visual inspection was conducted of the length of metal pipe (approximately 20 feet [ft] long) and the cylindrical metal debris. No other biased conditions were identified during the visual inspection. As a result, no additional samples were collected.

\subsubsection{Field Screening}

The radiological FSRs were compared to FSLs to guide subsequent sampling decisions. The radiological FSRs were all below FSLs. As a result, no additional samples were collected.

\subsubsection{Sample Collection}

A total of six soil environmental samples (including 1 FD and one MS/MSD) were collected from five locations at CAS 12-09-01 The sample identification numbers, locations, types, and analyses are listed in Table A.5-1. The sample locations are shown in Figure A.5-3. Samples were collected using scoops and a hand auger. Samples collected at this CAS are numbered 234C001 though 234C006.

\subsubsection{Conceptual Site Model Validation}

The CSM and associated discussion for this CAS are provided in the CAIP (NNSA/NSO, 2007). The information supporting the lack of contamination gathered during the CAI was consistent with the CSM, and all information gathered during the CAI supports and validates the CSM as presented in the CAIP (NNSA/NSO, 2007). 


\subsubsection{Mud Pit (CAS 12-09-08)}

The following subsections summarize the activities conducted at CAS 12-09-08.

\subsubsection{Radiological Survey}

An aerial radiological survey was performed in 1994 of Area 12, including CAS 12-09-08. The results of the survey were indistinguishable from background. As a result, no additional samples were collected (see Figure A.5-3).

\subsubsection{Visual Inspection}

A visual inspection was conducted of the CAS, and the only locations of environmental concern identified were the metal pipe sticking out of the ground at the top of one of the berm walls and a set of crushed 55-gal drums protruding from the eastern berm wall. The metal pipe was removed from the berm wall, placed on the ground, and the interior inspected. Nothing was identified within the pipe. No other items of concern were identified at the CAS. As a result, no additional samples were collected.

\subsubsection{Field Screening}

A handheld survey instrument was used to screen for alpha and beta/gamma radioactivity before soil samples were placed in sample jars. The radiological FSRs were compared to FSLs to guide subsequent sampling decisions. The radiological FSRs were all below FSLs.

\subsubsection{Sample Collection}

A total of seven soil environmental samples (including one FD and one MS/MSD) were collected from three locations at CAS 12-09-08. The sample identification numbers, locations, types, and analyses are listed in Table A.6-1. The sample locations are shown in Figure A.6-2. Samples were collected using scoops and a hand auger. Samples collected from this CAS are numbered 234D001 through 234D007. 


\subsubsection{Conceptual Site Model Validation}

The CSM and associated discussion for this CAS are provided in the CAIP (NNSA/NSO, 2007). The information supporting the lack of contamination gathered during the CAI was consistent with the CSM, and all information gathered during the CAI supports and validates the CSM as presented in the CAIP (NNSA/NSO, 2007).

\subsubsection{Cellar (CAS 12-30-14)}

The following subsections summarize the activities conducted at CAS 12-30-14.

\subsubsection{Radiological Survey}

An aerial radiological survey was conducted in 1994 of Area 12, including CAS 12-30-14. The findings of the survey were indistinguishable from background. Therefore, no additional samples were collected (see Figure A.5-2).

\subsubsection{Visual Inspection}

A visual inspection was conducted of the CAS, and the only locations of environmental concern identified were the open cellar and its contents. A metal pipe is sticking out of the cellar, but it was determined that this pipe had been placed in the cellar and was not an integral part of the cellar components. Visual inspection resulted in no additional collection of samples.

\subsubsection{Field Screening}

Soil samples were screened in the field for alpha and beta/gamma radioactivity. A handheld survey instrument was used to screen for alpha and beta/gamma radioactivity before soil samples were placed in sample jars. A liquid sample and all sediment samples were analyzed for shipping purposes using the gamma spectrometer located in Building 23-153. The radiological FSRs were compared to FSLs to guide subsequent sampling decisions. The radiological FSRs were all below FSLs.

\subsubsection{Sample Collection}

A total of one liquid and three sediment environmental samples (including one FD) were collected from two locations at CAS 12-30-14. The sample identification numbers, locations, types, and 
analyses are listed in Table A.7-1. The sample locations are shown in Figure A.7-2. Samples were collected using a Teflon beaker on a pole. The liquid sample was designated as 234E001, and the three sediment samples were designated 234E002 through 234E004.

\subsubsection{Conceptual Site Model Validation}

The CSM and associated discussion for this CAS are provided in the CAIP (NNSA/NSO, 2007).

The information supporting the lack of contamination gathered during the CAI was consistent with the CSM, and all information gathered during the CAI supports and validates the CSM as presented in the CAIP (NNSA/NSO, 2007).

\subsubsection{Summary of Analytical Data}

Chemical and radiological results for environmental and cellar content samples collected at each of the CASs with results greater than their respective minimum detectable concentrations (MDCs) are summarized in Sections A.3.0 through A.7.0. Environmental samples are evaluated against FALs to determine the presence of COCs and the extent of COC contamination, if present. The CAS 12-30-14 liquid sample results are evaluated against RCRA toxicity characteristics [TCs] to determine whether a release of the cellar contents to the surrounding environmental media could cause the presence of a $\mathrm{COC}$ in the environmental media.

The preliminary action levels (PALs) for the CAU 234 investigation were determined during the DQO process and are discussed in Section 3.3 of the CAIP (NNSA/NSO, 2007). The FALs used for determining the presence of COCs and for evaluating the need for additional corrective action are defined in Section 2.3. Details about the methods used during this investigation and a comparison of environmental sample results to the FALs are presented in Appendix A.

\subsection{Results}

\subsubsection{Summary of Analytical Data}

All concentrations of the reported parameters were compared to and were less than the PALs. The FALs were established at the corresponding PAL concentrations. No COCs were identified at any of 
the CASs and the CAS 12-30-14 cellar sample contents were less than the TC limits (i.e., no COCs identified).

The maximum concentration of each detected contaminant at CASs 02-09-48, 03-09-02, 12-09-01, 12-09-08, and 12-30-14 are listed in Tables 2-1 through 2-6, respectively.

\subsubsection{Data Assessment Summary}

The DQA is presented in Appendix B and includes an evaluation of the DQIs to determine the degree of acceptability and usability of the reported data in the decision-making process. The DQO process ensures that the right type, quality, and quantity of data will be available to support the resolution of those decisions at an appropriate level of confidence. Using both the DQO and DQA processes helps to ensure that DQO decisions are sound and defensible.

The DQA process as presented in Appendix B is comprised of the following steps:

- Step 1: Review DQOs and Sampling Design.

- Step 2: Conduct a Preliminary Data Review.

- Step 3: Select the Test.

- Step 4: Verify the Assumptions.

- Step 5: Draw Conclusions from the Data.

Sample locations that support the presence and/or extent of contamination at each CAS are shown in Appendix A. Based on the results of the DQA presented in Appendix B, the DQO requirements have been met. The DQA also determined that information generated during the investigation supports the CSM assumptions and the data collected support their intended use in the decision-making process.

\subsection{Justification for No Further Action}

No further corrective action is justified for all CAU 234 corrective action sites based on an evaluation of risk to ensure protection of the public and the environment in accordance with Nevada Administrative Code (NAC) 445A (NAC, 2006a), feasibility, and cost effectiveness. The decision that no further action is needed was determined from DQO decision statements based on a comparison of the analyte concentrations detected in CAI soil samples to the FALs defined in Section 2.3.1. 
Table 2-1

\section{Maximum Concentration of Detected Contaminants in Soil at} CAS 02-09-48, Area 2 Mud Plant \#1

\begin{tabular}{|c|c|c|c|c|c|c|}
\hline Constituent & $\begin{array}{c}\text { Maximum } \\
\text { Result }\end{array}$ & $\begin{array}{l}\text { Sample } \\
\text { Number }\end{array}$ & $\begin{array}{l}\text { Depth } \\
\text { (ft bgs) }\end{array}$ & Location & FAL & Units \\
\hline Actinium-228 & 3.72 & $234 \mathrm{~A} 005$ & $0.5-1.0$ & $\mathrm{~A} 02$ & 5 & $\mathrm{pCi} / \mathrm{g}$ \\
\hline Arsenic & 2.8 & $234 \mathrm{~A} 002$ & $0.0-0.5$ & $\mathrm{~A} 01$ & 23 & $\mathrm{mg} / \mathrm{kg}$ \\
\hline Barium & 100 & 234A001 & $0.0-0.5$ & $\mathrm{~A} 01$ & 67,000 & $\mathrm{mg} / \mathrm{kg}$ \\
\hline Benzo(b)Fluoranthene & $0.18(\mathrm{~J})$ & $234 \mathrm{~A} 002$ & $0.0-0.5$ & $\mathrm{~A} 01$ & 2.1 & $\mathrm{mg} / \mathrm{kg}$ \\
\hline Bis(2-ethylhexyl)Phthalate & $0.16(\mathrm{~J})$ & 234A005 & $0.5-1.0$ & $\mathrm{~A} 02$ & 120 & $\mathrm{mg} / \mathrm{kg}$ \\
\hline Cadmium & 0.65 & $234 \mathrm{~A} 001$ & $0.0-0.5$ & $\mathrm{~A} 01$ & 450 & $\mathrm{mg} / \mathrm{kg}$ \\
\hline Chromium & 3.8 & $234 \mathrm{~A} 001$ & $0.0-0.5$ & $\mathrm{~A} 01$ & 450 & $\mathrm{mg} / \mathrm{kg}$ \\
\hline Di-n-butyl Phthalate & $0.34(\mathrm{~J})$ & 234A002 & $0.0-0.5$ & $\mathrm{~A} 01$ & 62,000 & $\mathrm{mg} / \mathrm{kg}$ \\
\hline Fluoranthene & $0.28(\mathrm{~J})$ & 234A002 & $0.0-0.5$ & $\mathrm{~A} 01$ & 22,000 & $\mathrm{mg} / \mathrm{kg}$ \\
\hline Lead & 29 & 234A001 & $0.0-0.5$ & $\mathrm{~A} 01$ & 800 & $\mathrm{mg} / \mathrm{kg}$ \\
\hline Lead & 29 & $234 \mathrm{~A} 004$ & $0.0-0.5$ & $\mathrm{~A} 02$ & 800 & $\mathrm{mg} / \mathrm{kg}$ \\
\hline Lead & 29 & $234 \mathrm{~A} 003$ & $0.5-1.0$ & $\mathrm{~A} 01$ & 800 & $\mathrm{mg} / \mathrm{kg}$ \\
\hline Lead-212 & $4.12(\mathrm{~J})$ & $234 \mathrm{~A} 003$ & $0.5-1.0$ & $\mathrm{~A} 01$ & 5 & $\mathrm{pCi} / \mathrm{g}$ \\
\hline Lead-214 & $3.36(\mathrm{~J})$ & 234A005 & $0.5-1.0$ & A02 & 5 & $\mathrm{pCi} / \mathrm{g}$ \\
\hline Phenanthrene & $0.2(\mathrm{~J})$ & 234A002 & $0.0-0.5$ & $\mathrm{~A} 01$ & 100,000 & $\mathrm{mg} / \mathrm{kg}$ \\
\hline Plutonium-238 & 0.093 & $234 \mathrm{~A} 001$ & $0.0-0.5$ & $\mathrm{~A} 01$ & 13 & $\mathrm{pCi} / \mathrm{g}$ \\
\hline Plutonium-239/240 & 0.35 & $234 \mathrm{~A} 001$ & $0.0-0.5$ & $\mathrm{~A} 01$ & 12.7 & $\mathrm{pCi} / \mathrm{g}$ \\
\hline Pyrene & $0.2(\mathrm{~J})$ & 234A002 & $0.0-0.5$ & $\mathrm{~A} 01$ & 29,000 & $\mathrm{mg} / \mathrm{kg}$ \\
\hline Thorium-234 & $4.6(\mathrm{~J})$ & $234 \mathrm{~A} 005$ & $0.5-1.0$ & A02 & 105 & $\mathrm{pCi} / \mathrm{g}$ \\
\hline Thorium-234 & $4.6(\mathrm{~J})$ & $234 \mathrm{~A} 002$ & $0.0-0.5$ & $\mathrm{~A} 01$ & 105 & $\mathrm{pCi} / \mathrm{g}$ \\
\hline Thallium-208 & 1.22 & $234 \mathrm{~A} 004$ & $0.0-0.5$ & $\mathrm{~A} 02$ & 5 & $\mathrm{pCi} / \mathrm{g}$ \\
\hline Uranium-234 & 2.59 & $234 \mathrm{~A} 003$ & $0.5-1.0$ & $\mathrm{~A} 01$ & 143 & $\mathrm{pCi} / \mathrm{g}$ \\
\hline Uranium-235 & 0.16 & 234A002 & $0.0-0.5$ & $\mathrm{~A} 01$ & 17.6 & $\mathrm{pCi} / \mathrm{g}$ \\
\hline Uranium-238 & 2.66 & 234A005 & $0.5-1.0$ & A02 & 105 & $\mathrm{pCi} / \mathrm{g}$ \\
\hline
\end{tabular}

bgs $=$ Below ground surface

$\mathrm{FAL}=$ Final action level

$\mathrm{ft}=$ Foot

$\mathrm{mg} / \mathrm{kg}=$ Milligrams per kilogram

$\mathrm{pCi} / \mathrm{g}=$ Picocuries per gram

$\mathrm{J}=$ Estimated value 
Table 2-2

\section{Maximum Concentration of Detected Contaminants in Soil at CAS 03-09-02, Mud Dump Trenches}

\begin{tabular}{|c|c|c|c|c|c|c|}
\hline Constituent & $\begin{array}{c}\text { Maximum } \\
\text { Result }\end{array}$ & $\begin{array}{l}\text { Sample } \\
\text { Number }\end{array}$ & $\begin{array}{c}\text { Depth } \\
\text { (ft bgs) }\end{array}$ & Location & FAL & Units \\
\hline Actinium-228 & 3.49 & 234B014 & $0.0-0.5$ & B07 & 5 & $\mathrm{pCi} / \mathrm{g}$ \\
\hline Acetone & 0.11 & 234B005 & $1.5-2.0$ & B01 & 54,000 & $\mathrm{mg} / \mathrm{kg}$ \\
\hline Arsenic & 9.5 & 234B012 & $0.0-0.5$ & B06 & 23 & $\mathrm{mg} / \mathrm{kg}$ \\
\hline Barium & 310 & 234B012 & $0.0-0.5$ & B06 & 67,000 & $\mathrm{mg} / \mathrm{kg}$ \\
\hline Bis(2-ethylhexyl)Phthalate & $0.35(\mathrm{~J})$ & 234B004 & $1.5-2.0$ & B02 & 120 & $\mathrm{mg} / \mathrm{kg}$ \\
\hline Cadmium & 0.22 & 234B006 & $0.0-0.5$ & B03 & 450 & $\mathrm{mg} / \mathrm{kg}$ \\
\hline Chromium & 8.7 & 234B012 & $0.0-0.5$ & B06 & 450 & $\mathrm{mg} / \mathrm{kg}$ \\
\hline Cesium-137 & 2.7 & 234B008 & $0.0-0.5$ & B04 & 12.2 & $\mathrm{pCi} / \mathrm{g}$ \\
\hline Diesel-Range Organics & 53 & 234B012 & $0.0-0.5$ & B06 & 100 & $\mathrm{mg} / \mathrm{kg}$ \\
\hline Europium-155 & $0.274(\mathrm{~J})$ & 234B014 & $0.0-0.5$ & B07 & 135 & $\mathrm{pCi} / \mathrm{g}$ \\
\hline \multirow{2}{*}{ Lead } & 17 & 234B006 & $0.0-0.5$ & B03 & \multirow{2}{*}{800} & \multirow{2}{*}{$\mathrm{mg} / \mathrm{kg}$} \\
\hline & 17 & 234B014 & $0.0-0.5$ & B07 & & \\
\hline Mercury & 0.034 & 234B004 & $1.5-2.0$ & B02 & 310 & $\mathrm{mg} / \mathrm{kg}$ \\
\hline Lead-212 & $3.75(\mathrm{~J})$ & 234B014 & $0.0-0.5$ & B07 & 5 & $\mathrm{pCi} / \mathrm{g}$ \\
\hline Lead-214 & $1.44(\mathrm{~J})$ & 234B014 & $0.0-0.5$ & B07 & 5 & $\mathrm{pCi} / \mathrm{g}$ \\
\hline Plutonium-239/240 & 0.239 & 234B001 & $0.0-0.5$ & B01 & 12.7 & $\mathrm{pCi} / \mathrm{g}$ \\
\hline Selenium & 0.55 & 234B004 & $1.5-2.0$ & B02 & 5,100 & $\mathrm{mg} / \mathrm{kg}$ \\
\hline Thallium-208 & 1.14 & 234B014 & $0.0-0.5$ & B07 & 5 & $\mathrm{pCi} / \mathrm{g}$ \\
\hline Thorium-234 & $4.72(\mathrm{~J})$ & 234B014 & $0.0-0.5$ & B07 & 105 & $\mathrm{pCi} / \mathrm{g}$ \\
\hline Uranium-234 & 1.44 & 234B008 & $0.0-0.5$ & B04 & 143 & $\mathrm{pCi} / \mathrm{g}$ \\
\hline Uranium-235 & 0.094 & 234B003 & $0.0-0.5$ & B02 & 17.6 & $\mathrm{pCi} / \mathrm{g}$ \\
\hline Uranium-238 & 1.56 & 234B008 & $0.0-0.5$ & B04 & 105 & $\mathrm{pCi} / \mathrm{g}$ \\
\hline
\end{tabular}

bgs $=$ Below ground surface

$\mathrm{FAL}=$ Final action level

$\mathrm{ft}=$ Foot

$\mathrm{mg} / \mathrm{kg}=$ Milligrams per kilogram

$\mathrm{pCi} / \mathrm{g}=$ Picocuries per gram

$\mathrm{J}=$ Estimated value 
Table 2-3

\section{Maximum Concentration of Detected Contaminants in Soil at CAS 12-09-01, Mud Pit}

\begin{tabular}{|c|c|c|c|c|c|c|}
\hline Constituent & $\begin{array}{c}\text { Maximum } \\
\text { Result }\end{array}$ & $\begin{array}{l}\text { Sample } \\
\text { Number }\end{array}$ & $\begin{array}{c}\text { Depth } \\
\text { (ft bgs) }\end{array}$ & Location & FAL & Units \\
\hline Actinium-228 & 2.64 & $234 \mathrm{C} 001$ & $0.0-0.33$ & $\mathrm{C} 01$ & 5 & $\mathrm{pCi} / \mathrm{g}$ \\
\hline Arsenic & 3.7 & 234C006 & $0.0-0.5$ & $\mathrm{C} 05$ & 23 & $\mathrm{mg} / \mathrm{kg}$ \\
\hline Barium & 120 & $234 \mathrm{C} 001$ & $0.0-0.33$ & $\mathrm{C} 01$ & 67,000 & $\mathrm{mg} / \mathrm{kg}$ \\
\hline Barium & 120 & $234 \mathrm{C} 002$ & $0.0-0.33$ & $\mathrm{C} 01$ & 67,000 & $\mathrm{mg} / \mathrm{kg}$ \\
\hline Benzo(b)Fluoranthene & $0.086(J)$ & $234 \mathrm{C} 002$ & $0.0-0.33$ & C01 & 2 & $\mathrm{mg} / \mathrm{kg}$ \\
\hline Bis(2-ethylhexyl)Phthalate & $0.12(\mathrm{~J})$ & $234 \mathrm{C} 001$ & $0.0-0.33$ & C01 & 120 & $\mathrm{mg} / \mathrm{kg}$ \\
\hline Cadmium & 0.14 & $234 \mathrm{C} 006$ & $0.0-0.5$ & $\mathrm{C} 05$ & 450 & $\mathrm{mg} / \mathrm{kg}$ \\
\hline Chromium & 7.4 & $234 \mathrm{C} 006$ & $0.0-0.5$ & $\mathrm{C} 05$ & 450 & $\mathrm{mg} / \mathrm{kg}$ \\
\hline Cesium-137 & 0.45 & $234 \mathrm{C} 001$ & $0.0-0.33$ & $\mathrm{C} 01$ & 12 & $\mathrm{pCi} / \mathrm{g}$ \\
\hline Diesel-Range Organics & 7 & $234 \mathrm{C} 001$ & $0.0-0.33$ & $\mathrm{C} 01$ & 100 & $\mathrm{mg} / \mathrm{kg}$ \\
\hline Lead & $33(\mathrm{~J})$ & $234 \mathrm{C} 006$ & $0.0-0.5$ & C05 & 800 & $\mathrm{mg} / \mathrm{kg}$ \\
\hline Mercury & 0.026 & $234 \mathrm{C} 005$ & $0.0-0.5$ & $\mathrm{C} 04$ & 310 & $\mathrm{mg} / \mathrm{kg}$ \\
\hline p-Isopropyltoluene & $0.0022(\mathrm{~J})$ & 234C004 & $0.0-0.5$ & $\mathrm{CO3}$ & 2,000 & $\mathrm{mg} / \mathrm{kg}$ \\
\hline Lead-212 & $2.87(\mathrm{~J})$ & $234 \mathrm{C} 001$ & $0.0-0.33$ & $\mathrm{C} 01$ & 5 & $\mathrm{pCi} / \mathrm{g}$ \\
\hline Lead-214 & $1.55(\mathrm{~J})$ & $234 \mathrm{C} 003$ & $0.0-0.33$ & C02 & 5 & $\mathrm{pCi} / \mathrm{g}$ \\
\hline Plutonium-238 & 0.13 & $234 \mathrm{C} 005$ & $0.0-0.5$ & $\mathrm{CO4}$ & 13 & $\mathrm{pCi} / \mathrm{g}$ \\
\hline Plutonium-239/240 & 0.66 & $234 \mathrm{C} 005$ & $0.0-0.5$ & $\mathrm{CO4}$ & 13 & $\mathrm{pCi} / \mathrm{g}$ \\
\hline Selenium & 0.46 & $234 \mathrm{C} 002$ & $0.0-0.33$ & $\mathrm{C} 01$ & 5,100 & $\mathrm{mg} / \mathrm{kg}$ \\
\hline Silver & 0.2 & $234 \mathrm{C} 002$ & $0.0-0.33$ & $\mathrm{C} 01$ & 5,100 & $\mathrm{mg} / \mathrm{kg}$ \\
\hline Thallium-208 & 0.95 & $234 \mathrm{C} 003$ & $0.0-0.33$ & $\mathrm{C} 02$ & 5 & $\mathrm{pCi} / \mathrm{g}$ \\
\hline Uranium-234 & 1.27 & $234 \mathrm{C} 001$ & $0.0-0.33$ & $\mathrm{C} 01$ & 143 & $\mathrm{pCi} / \mathrm{g}$ \\
\hline Uranium-235 & 0.08 & $234 \mathrm{C} 003$ & $0.0-0.33$ & $\mathrm{C} 02$ & 18 & $\mathrm{pCi} / \mathrm{g}$ \\
\hline Uranium-235 & 0.08 & $234 \mathrm{C} 004$ & $0.0-0.5$ & $\mathrm{CO3}$ & 18 & $\mathrm{pCi} / \mathrm{g}$ \\
\hline Uranium-238 & 1.3 & $234 \mathrm{C} 005$ & $0.0-0.5$ & $\mathrm{CO4}$ & 105 & $\mathrm{pCi} / \mathrm{g}$ \\
\hline
\end{tabular}

bgs $=$ Below ground surface

$\mathrm{FAL}=$ Final action level

$\mathrm{ft}=$ Foot

$\mathrm{mg} / \mathrm{kg}=$ Milligrams per kilogram

$\mathrm{pCi} / \mathrm{g}=$ Picocuries per gram

$\mathrm{J}=$ Estimated value 
Table 2-4

\section{Maximum Concentration of Detected Contaminants in Soil at CAS 12-09-08, Mud Pit}

\begin{tabular}{||c|c|c|c|c|c|c||}
\hline Constituent & $\begin{array}{c}\text { Maximum } \\
\text { Result }\end{array}$ & $\begin{array}{c}\text { Sample } \\
\text { Number }\end{array}$ & $\begin{array}{c}\text { Depth } \\
\text { (ft bgs) }\end{array}$ & Location & FAL & Units \\
\hline \hline Actinium-228 & 2.56 & $234 \mathrm{D} 001$ & $0.0-0.5$ & $\mathrm{D} 01$ & 5 & $\mathrm{pCi} / \mathrm{g}$ \\
\hline Arsenic & 4.5 & $234 \mathrm{D} 001$ & $0.0-0.5$ & $\mathrm{D} 01$ & 23 & $\mathrm{mg} / \mathrm{kg}$ \\
\hline Barium & 200 & $234 \mathrm{D} 006$ & $0.0-0.5$ & $\mathrm{D} 03$ & 67,000 & $\mathrm{mg} / \mathrm{kg}$ \\
\hline Bis(2-ethylhexyl)Phthalate & $0.18(\mathrm{~J})$ & $234 \mathrm{D} 007$ & $1.0-1.5$ & $\mathrm{D} 03$ & 120 & $\mathrm{mg} / \mathrm{kg}$ \\
\hline Cadmium & 0.19 & $234 \mathrm{D} 005$ & $0.0-0.5$ & $\mathrm{D} 03$ & 450 & $\mathrm{mg} / \mathrm{kg}$ \\
\hline Chromium & 7 & $234 \mathrm{D} 001$ & $0.0-0.5$ & $\mathrm{D} 01$ & 450 & $\mathrm{mg} / \mathrm{kg}$ \\
\hline Diesel-Range Organics & 73 & $234 \mathrm{D} 007$ & $1.0-1.5$ & $\mathrm{D} 03$ & 100 & $\mathrm{mg} / \mathrm{kg}$ \\
\hline Lead & 22 & $234 \mathrm{D} 006$ & $0.0-0.5$ & $\mathrm{D} 03$ & 800 & $\mathrm{mg} / \mathrm{kg}$ \\
\hline Lead-212 & $2.91(\mathrm{~J})$ & $234 \mathrm{D} 007$ & $1.0-1.5$ & $\mathrm{D} 03$ & 5 & $\mathrm{pCi} / \mathrm{g}$ \\
\hline Lead-214 & $1.26(\mathrm{~J})$ & $234 \mathrm{D} 007$ & $1.0-1.5$ & $\mathrm{D} 03$ & 5 & $\mathrm{pCi} / \mathrm{g}$ \\
\hline Plutonium-239/240 & 0.028 & $234 \mathrm{D} 005$ & $0.0-0.5$ & $\mathrm{D} 03$ & 12.7 & $\mathrm{pCi} / \mathrm{g}$ \\
\hline Selenium & 0.46 & $234 \mathrm{D} 002$ & $0.5-1.0$ & $\mathrm{D} 01$ & 5,100 & $\mathrm{mg} / \mathrm{kg}$ \\
\hline Thallium-208 & 0.89 & $234 \mathrm{D} 002$ & $0.5-1.0$ & $\mathrm{D} 01$ & 5 & $\mathrm{pCi} / \mathrm{g}$ \\
\hline Uranium-234 & 1.06 & $234 \mathrm{D} 003$ & $0.0-0.5$ & $\mathrm{D} 02$ & 143 & $\mathrm{pCi} / \mathrm{g}$ \\
\hline Uranium-235 & 0.081 & $234 \mathrm{D} 005$ & $0.0-0.5$ & $\mathrm{D} 03$ & 17.6 & $\mathrm{pCi} / \mathrm{g}$ \\
\hline Uranium-238 & 1.09 & $234 \mathrm{D} 004$ & $0.5-1.0$ & $\mathrm{D} 02$ & 105 & $\mathrm{pCi} / \mathrm{g}$ \\
\hline Uranium-238 & 1.09 & $234 \mathrm{D} 003$ & $0.0-0.5$ & $\mathrm{D} 02$ & 105 & $\mathrm{pCi} / \mathrm{g}$ \\
\hline
\end{tabular}

bgs $=$ Below ground surface

$\mathrm{FAL}=$ Final action level

$\mathrm{ft}=$ Foot

$\mathrm{mg} / \mathrm{kg}=$ Milligrams per kilogram

$\mathrm{pCi} / \mathrm{g}=$ Picocuries per gram

$\mathrm{J}=$ Estimated value

No contaminants were identified at concentrations exceeding their respective FALs (or the TC limit for the CAS 12-30-14 liquid cellar samples) in any of the CASs.

As no COCs were identified, no corrective action is required. Appendix $\mathrm{C}$ presents the justification for no further action based on risk. 
Table 2-5

\section{Maximum Concentration of Detected Sediment Contaminants for CAS 12-30-14, Cellar}

\begin{tabular}{|c|c|c|c|c|c|c|}
\hline Constituent & $\begin{array}{l}\text { Maximum } \\
\text { Result }\end{array}$ & $\begin{array}{l}\text { Sample } \\
\text { Number }\end{array}$ & Thickness $^{\mathrm{a}}$ & Location & FAL & Units \\
\hline Uranium-234 & 0.97 & 234E002 & $0.0-1.0$ & Cellar & 143 & $\mathrm{pCi} / \mathrm{g}$ \\
\hline Actinium-228 & 1.83 & 234E002 & $0.0-1.0$ & Cellar & 5 & $\mathrm{pCi} / \mathrm{g}$ \\
\hline Thallium-208 & 0.66 & 234E002 & $0.0-1.0$ & Cellar & 5 & $\mathrm{pCi} / \mathrm{g}$ \\
\hline Lead-214 & 1.48 & 234E002 & $0.0-1.0$ & Cellar & 5 & $\mathrm{pCi} / \mathrm{g}$ \\
\hline Lead-212 & 2.19 & 234E002 & $0.0-1.0$ & Cellar & 5 & $\mathrm{pCi} / \mathrm{g}$ \\
\hline Uranium-235 & 0.053 & 234E003 & $0.0-1.0$ & Cellar & 17.6 & $\mathrm{pCi} / \mathrm{g}$ \\
\hline Uranium-238 & 1.03 & 234E004 & $0.0-1.0$ & Cellar & 105 & $\mathrm{pCi} / \mathrm{g}$ \\
\hline Bis(2-ethylhexyl)Phthalate & 0.64 & 234E004 & $0.0-1.0$ & Cellar & 120 & $\mathrm{mg} / \mathrm{kg}$ \\
\hline Acetone & 0.059 & 234E002 & $0.0-1.0$ & Cellar & 54,000 & $\mathrm{mg} / \mathrm{kg}$ \\
\hline p-Isopropyltoluene & 0.16 & 234E002 & $0.0-1.0$ & Cellar & 2,000 & $\mathrm{mg} / \mathrm{kg}$ \\
\hline Diesel-Range Organics & 60 & 234E002 & $0.0-1.0$ & Cellar & 100 & $\mathrm{mg} / \mathrm{kg}$ \\
\hline Lead & 210 & 234E002 & $0.0-1.0$ & Cellar & 800 & $\mathrm{mg} / \mathrm{kg}$ \\
\hline Arsenic & 5 & 234E004 & $0.0-1.0$ & Cellar & 23 & $\mathrm{mg} / \mathrm{kg}$ \\
\hline Barium & 3,100 & 234E002 & $0.0-1.0$ & Cellar & 67,000 & $\mathrm{mg} / \mathrm{kg}$ \\
\hline Cadmium & 1 & 234E002 & $0.0-1.0$ & Cellar & 450 & $\mathrm{mg} / \mathrm{kg}$ \\
\hline Chromium & 6.3 & 234E002 & $0.0-1.0$ & Cellar & 450 & $\mathrm{mg} / \mathrm{kg}$ \\
\hline Selenium & 0.62 & 234E004 & $0.0-1.0$ & Cellar & 5,100 & $\mathrm{mg} / \mathrm{kg}$ \\
\hline
\end{tabular}

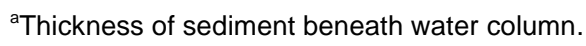

$\mathrm{FAL}=$ Final action level

$\mathrm{ft}=$ Foot

$\mathrm{mg} / \mathrm{kg}=$ Milligrams per kilogram

$\mathrm{pCi} / \mathrm{g}=$ Picocuries per gram

As a BMP, housekeeping will be performed and documented in the final version of this document for those CASs where debris was encountered. Specifically, the items to be removed are as follows:

- CAS 02-09-48 (Area 2 Mud Plant \#1): removal of the rusted 55-gal barrel from the mud sump.

- CAS 03-09-02 (Mud Dump Trenches): removal of blue pipe from within the suction pit. 
Table 2-6

\section{Maximum Concentration of Detected Liquid Contaminants for CAS 12-30-14, Cellar}

\begin{tabular}{|c|c|c|c|c|c|c|}
\hline Constituent & $\begin{array}{c}\text { Maximum } \\
\text { Result }\end{array}$ & $\begin{array}{l}\text { Sample } \\
\text { Number }\end{array}$ & $\begin{array}{c}\text { Thickness }^{a} \\
\text { (ft bgs) }\end{array}$ & Location & $\begin{array}{c}\text { PSM } \\
\text { Criteria }^{b}\end{array}$ & Units \\
\hline Strontium-90 & 3.05 & 234E001 & $0.0-1.5$ & Cellar & $\mathrm{N} / \mathrm{A}$ & $\overline{\mathrm{pCi} / \mathrm{L}}$ \\
\hline Acetone & 0.064 & 234E001 & $0.0-1.5$ & Cellar & None & $\mathrm{mg} / \mathrm{L}$ \\
\hline Lead & 0.002 & 234E001 & $0.0-1.5$ & Cellar & 5.0 & $\mathrm{mg} / \mathrm{L}$ \\
\hline Arsenic & 0.0082 & 234E001 & $0.0-1.5$ & Cellar & 5.0 & $\mathrm{mg} / \mathrm{L}$ \\
\hline Barium & 0.15 & 234E001 & $0.0-1.5$ & Cellar & 100.0 & $\mathrm{mg} / \mathrm{L}$ \\
\hline
\end{tabular}

${ }^{a}$ Thickness of water column above sediment on cellar floor.

${ }^{b}$ See Section A.7.2.

bgs $=$ Below ground surface

$\mathrm{ft}=$ Foot

$\mathrm{mg} / \mathrm{L}=$ Milligrams per liter

$\mathrm{N} / \mathrm{A}=$ Not applicable

$\mathrm{pCi} / \mathrm{L}=$ Picocuries per liter

$\mathrm{PSM}=$ Potential source material

- CAS 12-09-01 (Mud Pit): removal of the piece of metal pipe lying on the ground and the cylindrical metal debris.

- CAS 12-09-08 (Mud Pit): removal of the short length of pipe laying on the berm wall and the three crushed 55-gal drums located at the berm wall mud pit interface.

\subsubsection{Final Action Levels}

The CAU 234 FALs are risk-based cleanup goals that, if met, will ensure that each release site will not pose an unacceptable risk to human health and the environment and that conditions at each site are in compliance with all applicable laws and regulations. The risk-based corrective action (RBCA) process used to establish FALs is described in the Industrial Sites Project Establishment of Final Action Levels (NNSA/NSO, 2006). This process conforms with NAC Section 445A.227, which lists the requirements for sites with soil contamination (NAC, 2006b). For the evaluation of corrective actions, NAC Section 445A.22705 (NAC, 2006c) requires the use of American Society for Testing and Materials (ASTM) Method E 1739-95 (ASTM, 1995) to "conduct an evaluation of the site, based on the risk it poses to public health and the environment, to determine the necessary remediation standards (i.e., FALs) or to establish that corrective action is not necessary." 
This RBCA process defines three tiers (or levels) of evaluation involving increasingly sophisticated analyses:

- Tier I evaluation - Sample results from source areas (highest concentrations) are compared to action levels based on generic (non-site-specific) conditions (i.e., the PALs established in the CAIP [NNSA/NSO, 2007]). The FALs may then be established as the Tier I action levels or calculated using a Tier II evaluation.

- Tier II evaluation - Conducted by calculating Tier II Site-Specific Target Levels (SSTLs) using site-specific information as inputs to the same or similar methodology used to calculate Tier I action levels. The Tier II SSTLs are then compared to individual sample results from reasonable points of exposure (as opposed to the source areas as is done in Tier I) on a point-by-point basis. Total concentrations of total petroleum hydrocarbons (TPH) will not be used for risk-based decisions under Tier II or Tier III. Rather, the individual chemicals of concern will be compared to the SSTLs.

- Tier III evaluation - Conducted by calculating Tier III SSTLs on the basis of more sophisticated risk analyses using methodologies described in Method E 1739-95 that consider site-, pathway-, and receptor-specific parameters.

A Tier I evaluation was conducted for all COPCs to determine whether contaminant levels satisfy the criteria for a quick regulatory closure or warrant a more site-specific assessment. This was accomplished by comparing individual source area contaminant concentration results to the Tier I action levels (the PALs established in the CAIP [NNSA/NSO, 2007]).

A Tier II evaluation was not required because all analytical results were below the risk-based screening level (RBSL) established at the Tier I level (i.e., results were all less than their respective PALs).

The FALs for all CAU 234 COPCs are shown in Table 2-7. 
Table 2-7

Definition of Final Action Levels for CAU 234 Contaminants of Potential Concern

\begin{tabular}{|c|c|c|c||}
\hline COPCs & Tier I-Based FALs & Tier II-Based FALs & Tier III-Based FALs \\
\hline \hline VOCs & All CASs & None & $\mathrm{N} / \mathrm{A}$ \\
\hline SVOCs & All CASs & None & $\mathrm{N} / \mathrm{A}$ \\
\hline PCBs & All CASs & None & $\mathrm{N} / \mathrm{A}$ \\
\hline RCRA metals & All CASs & None & $\mathrm{N} / \mathrm{A}$ \\
\hline TPH-DRO & All CASs & None & $\mathrm{N} / \mathrm{A}$ \\
\hline Radionuclides & All CASs & None & $\mathrm{N} / \mathrm{A}$ \\
\hline
\end{tabular}

$\mathrm{COPC}=$ Contaminant of potential concern DRO = Diesel-range organics

$\mathrm{FAL}=$ Final action level

$\mathrm{N} / \mathrm{A}=$ Not applicable

$\mathrm{PCB}=$ Polychlorinated biphenyl
RCRA = Resource Conservation and Recovery Act SVOC = Semivolatile organic compound

$\mathrm{TPH}=$ Total petroleum hydrocarbons

VOC $=$ Volatile organic compound 


\subsection{Recommendation}

No further corrective action is required at CAU 234. Selection of this corrective action is consistent with past practices for CASs that do not contain COCs. No further action was evaluated based on technical merits focusing on performance, reliability, feasibility, and safety. Debris removal will be conducted as a BMP and documented in the final version of this document.

The DOE, National Nuclear Security Administration Nevada Site Office (NNSA/NSO) requests that NDEP issues a Notice of Completion for this CAU and approval to move the CAU from Appendix III to Appendix IV of the FFACO. 


\subsection{References}

ASTM, see American Society for Testing and Materials.

American Society for Testing and Materials. 1995. Standard Guide for Risk-Based Corrective Action Applied at Petroleum Release Sites, ASTM E 1739-95 (Reapproved 2002). Philadelphia, PA.

CFR, see Code of Federal Regulations.

Code of Federal Regulations. 2006a. Title 40 CFR Parts 260 - 282, "Hazardous Waste Management." Washington, DC: U.S. Government Printing Office.

Code of Federal Regulations. 2006b. Title 40 CFR Part 761, "Polychlorinated Biphenyls (PCBs) Manufacturing, Processing, Distribution in Commerce and Prohibitions." Washington, DC: U.S. Government Printing Office.

EPA, see U.S. Environmental Protection Agency.

FFACO, see Federal Facility Agreement and Consent Order.

Federal Facility Agreement and Consent Order. 1996 (as amended February 2008). Agreed to by the State of Nevada; U.S. Department of Energy, Environmental Management; U.S. Department of Defense; and U.S. Department of Energy, Legacy Management.

NAC, see Nevada Administrative Code.

NNSA/NSO, see U.S. Department of Energy, National Nuclear Security Administration Nevada Site Office.

NNSA/NV, see U.S. Department of Energy, National Nuclear Security Administration Nevada Operations Office.

Nevada Administrative Code. 2006a. NAC 445A, "Water Controls." Carson City, NV. As accessed at http://www.leg.state.nv.us/nac on 29 January 2008.

Nevada Administrative Code. 2006b. NAC 445A.227, "Contamination of Soil: Order by Director for Corrective Action; Factors To Be Considered in Determining Whether Corrective Action Required." Carson City, NV. As accessed at http://www.leg.state.nv.us/nac on 29 January 2008.

Nevada Administrative Code. 2006c. NAC 445A.22705, "Contamination of Soil: Evaluation of Site by Owner or Operator; Review of Evaluation by Division." Carson City, NV. As accessed at http://www.leg.state.nv.us/nac on 29 January 2008. 
SNJV GIS, see Stoller-Navarro Joint Venture Geographic Information Systems.

Stoller-Navarro Joint Venture Geographic Information Systems. 2007. ESRI ArcGIS Software.

U.S. Department of Energy, National Nuclear Security Administration Nevada Operations Office. 2002. Industrial Sites Quality Assurance Project Plan, Nevada Test Site, Nevada, Rev. 3, DOE/NV--372. Las Vegas, NV.

U.S. Department of Energy, National Nuclear Security Administration Nevada Site Office. 2006. Industrial Sites Project Establishment of Final Action Levels, Rev. 0, DOE/NV--1107. Las Vegas, NV.

U.S. Department of Energy, National Nuclear Security Administration Nevada Site Office. 2007. Corrective Action Investigation Plan for Corrective Action Unit 234: Mud Pits, Cellars, and Mud Spills, Nevada Test Site, Nevada, Rev. 0, DOE/NV--1216. Las Vegas, NV.

U.S. Environmental Protection Agency. 2006. EPA Guidance on Systematic Planning Using the Data Quality Objectives Process, EPA QA/G-4. Washington, DC. 


\section{Appendix A}

\section{Corrective Action Investigation Results}




\section{A.1.0 Introduction}

This appendix presents the CAI activities and analytical results for CAU 234. Corrective Action Unit 234 is located in Areas 2, 3, 4, 12 and 15 of the NTS (Figure 1-1), and is comprised of the following 12 CASs:

- 02-09-48, Area 2 Mud Pit \#1

- 02-09-49, Area 2 Mud Pit \#2

- 02-99-05, Mud Spill

- 03-09-02, Mud Dump Trenches

- 04-44-02, Mud Spill

- 04-99-02, Mud Spill

- 12-09-01, Mud Pit

- 12-09-04, Mud Pit

- 12-09-08, Mud Pit

- 12-30-14, Cellar

- 12-99-07, Mud Dump

- 15-09-01, Mud Pit

Seven CASs — 02-09-49, 02-99-05, 04-44-02, 04-99-02, 12-09-04, 12-99-07, and 15-09-01 - are not included in the investigation for the reasons described in Section 1.1. These CASs meet the criteria defined in the Closure Report for Corrective Action Units 530, 531, 532, 533, 534, 535: NTS Mud Pits, Nevada Test Site, Nevada (NNSA/NSO, 2006a). The criteria allow mud pits or spills to be exempt from investigation if they are of the type described in the CR. These seven CASs meet the criteria.

The remaining five CASs (02-09-48, 03-09-02, 12-09-01, 12-09-08, and 12-30-14) were investigated because they contained debris or are associated with process knowledge that indicates potential presence of COPCs not commonly associated with mud pits.

Corrective Action Site 02-09-48 is located in Area 2 of the NTS and consists of a concrete-lined drilling mud sump used for storing unused drilling mud until needed for use. Some unused drilling mud remains in the sump. A 55-gal, rusted barrel sat on the drilling mud in the northwest corner of the sump. 
Corrective Action Site 03-09-02 is located in Area 3 of the NTS and consists of a complex of mud pits, suction pits, and mud trenches. The system of pits and trenches was used for the drilling of emplacement hole U-3kz in June 1984. The northern section of the CAS contains a large mud pit, a suction pit, and a reserve suction pit. The southern section of the CAS contains two trenches, one elevated relative to the other and connected by a trench, that are carved out of what appears to have been a borrow pit.

Corrective Action Site 12-09-01 is located in Area 12 of the NTS and consists of a mud pit associated with the drilling of the U-12r PS \#1A post-test cellar. Located within this CAS is a length of metal piping lying on the ground surface near the mud pit and a cylindrical piece of metal debris lying on its side, approximately $3.5 \mathrm{ft}$ in diameter, open at one end, with an opening on the side where a hinged door used to be attached.

Corrective Action Site 12-09-08 is located in Area 12 of the NTS and consists of a mud pit associated with the drilling of the U12e.14 HFR CH\#1 instrument hole that began on November 9, 1972. The mud pit contains a length of metal pipe protruding from the southwest corner of the berm by approximately $4 \mathrm{ft}$ and at an approximate 45-degree angle. There are several crushed 55-gal drums located on the inner slope of the mud pit berm along the western edge.

Corrective Action Site 12-30-14 is located in Area 12 of the NTS and consists of an open 10-ft-diameter cellar used for the drilling of the U12r PS\#1A and U12r PS\#1AS post-test boreholes. The cellar is approximately $9 \mathrm{ft}$ deep and is cased with corrugated metal. The post-test boreholes were drilled between January 19 and 24, 1969, to depths of approximately 2,000 ft below ground surface (bgs). Liquid resides within the cellar, and the depth fluctuates depending on precipitation and evaporation rates.

Additional information regarding the history of each site, planning, and the scope of the investigation is presented in the CAU 234 CAIP (NNSA/NSO, 2007).

\section{A.1.1 Project Objectives}

The primary objective of the investigation was to provide sufficient information to document completion of appropriate corrective actions for each CAS in CAU 234 to support a recommendation 
for closure of the CASs in CAU 234. This objective was achieved by identifying the absence or presence of COCs and the vertical and lateral extent of the COCs, if present.

\section{A.1.2 Contents}

This appendix describes the investigation and presents the results. The contents of this appendix are as follows:

- Section A.1.0 describes the investigation background, objectives, and content.

- Section A.2.0 provides an investigation overview.

- Sections A.3.0 through A.7.0 provide CAS-specific information regarding the field activities, sampling methods, and laboratory analytical results from investigation sampling.

- Section A.8.0 summarizes waste management activities.

- Section A.9.0 discusses the QA and QC processes followed and the results of QA/QC activities.

- Section A.10.0 provides a summary of the investigation results.

- Section A.11.0 lists the cited references.

The complete field documentation and laboratory data — including field activity daily logs, sample collection logs (SCLs), analysis request/chain-of-custody forms, soil sample descriptions, laboratory certificates of analyses, analytical results, and surveillance results — are retained in project files as hard copy files or electronic media. 


\section{A.2.0 Investigation Overview}

Field investigation and sampling activities for the CAU 234 CAI were conducted from October 31 through November 7, 2007. An additional sample was collected on January 23, 2008, from CAS 03-09-02. Table A.2-1 lists the CAI activities that were conducted at each of the CASs.

Table A.2-1

Corrective Action Investigation Activities Conducted at Each Corrective Action Site To Meet Corrective Action Investigation Plan Requirements for CAU 234

\begin{tabular}{|c|c|c|c|c|c|}
\hline \multirow[b]{2}{*}{ Corrective Action Investigation Activities } & \multicolumn{5}{|c|}{ Corrective Action Site } \\
\hline & $02-09-48$ & 03-09-02 & $12-09-01$ & $12-09-08$ & $12-30-14$ \\
\hline $\begin{array}{l}\text { Inspected and verified the CAS components identified in } \\
\text { the Corrective Action Investigation Plan. }\end{array}$ & $x$ & $x$ & $x$ & $\mathrm{X}$ & $x$ \\
\hline $\begin{array}{l}\text { Performed site walkovers to identify biased sampling } \\
\text { locations. }\end{array}$ & $x$ & $x$ & $x$ & $x$ & $x$ \\
\hline $\begin{array}{l}\text { Conducted scanning radiological walkover surveys } \\
\text { (i.e., soil, concrete surfaces, debris) using a handheld } \\
\text { detector and a global positioning system (GPS) receiver } \\
\text { with a TSCITM data logger. }\end{array}$ & $x$ & $x$ & $\mathrm{X}$ & $x$ & $\mathrm{X}$ \\
\hline Collected biased soil samples. & $\mathrm{X}$ & $X$ & $X$ & $\mathrm{X}$ & $\mathrm{X}$ \\
\hline $\begin{array}{l}\text { Field screened samples for alpha and beta/gamma } \\
\text { radiation using a handheld survey instrument. }\end{array}$ & $X$ & $x$ & $\mathrm{X}$ & $x$ & $x$ \\
\hline $\begin{array}{l}\text { Analyzed samples for gamma radiation using a } \\
\text { high-purity germanium gamma spectrometer } \\
\text { (Building 23-153, Mercury, NV). }\end{array}$ & -- & -- & -- & -- & $X$ \\
\hline $\begin{array}{l}\text { Collected liquid and sediment samples from the } \\
\text { contents of the cellar for waste characterization to } \\
\text { support disposal recommendations and determine } \\
\text { whether the waste could be a potential source of } \\
\text { contamination for the environment (i.e., soil). }\end{array}$ & -- & -- & -- & -- & $x$ \\
\hline Submitted select samples for offsite laboratory analysis. & $\mathrm{X}$ & $\mathrm{X}$ & $X$ & $\mathrm{X}$ & $\mathrm{X}$ \\
\hline $\begin{array}{l}\text { Collected GPS coordinates for sample locations and } \\
\text { points of interest. }\end{array}$ & $\mathrm{X}$ & $X$ & $\mathrm{X}$ & $\mathrm{X}$ & $\mathrm{X}$ \\
\hline
\end{tabular}

-- = Not applicable 
The investigation and sampling program was managed in accordance with the requirements set forth in the CAU 234 CAIP (NNSA/NSO, 2007). Samples were collected and documented following the CAU 234 CAIP. Quality control samples (e.g., field blanks, equipment rinsate blanks, trip blanks, and duplicate samples) were collected as required by the Industrial Sites QAPP (NNSA/NV, 2002) and the CAU 234 CAIP. During field activities, waste minimization practices were conducted according to approved procedures, including segregation of waste by waste type.

Weather conditions at the sites varied to include sun (moderate to low temperatures), no rainfall during sampling activities, intermittent cloudiness, and light winds.

The CASs were investigated by conducting site inspections, radiological surface screenings, and surveys; performing sampling of potential contaminant sources; and sampling surface and subsurface soils. Surface soil samples were collected by hand excavation. Subsurface soil samples were collected using hand augering. The soil samples were field screened at specific locations for alpha and beta/gamma radiation, and gamma-emitting radionuclides. The results were compared against screening levels to guide in the CAS-specific investigations. Samples of various media (e.g., soil, liquids, sediments) were collected to support both environmental and waste characterization using hand augers and plastic scoops.

Except as noted in the following CAS-specific sections, CAU 234 Decision I sampling locations were accessible, and sampling activities at planned locations were not restricted.

Sections A.2.1 through A.2.4 provide the investigation methodology, site geology and hydrology, and laboratory analytical information.

\section{A.2.1 Sample Locations}

Investigation locations selected for sampling were based on interpretation of existing engineering drawings, aerial and land photographs, interviews with former and current site employees, information obtained during site visits, and site conditions as provided in the CAU 234 CAIP (NNSA/NSO, 2007). The CAS-specific sampling points were selected based on physical characteristics of the CAS and the presence of debris. The planned biased sample locations (e.g., locations beneath debris) are discussed in text and represented on figures in the CAIP. Actual 
environmental sample locations are shown on the figures included in Sections A.3.0 through A.7.0. Sample locations were staked where appropriate and labeled. A Trimble Geo-XT GPS instrument was used for determining the sample location coordinates as well as CAS points of interest. Appendix E presents these data in a CAS-specific figure format.

\section{A.2.2 Investigation Activities}

The investigation activities listed in Table A.2-1 were performed at CAU 234 consistent with the field investigation activities stipulated in the CAU 234 CAIP (NNSA/NSO, 2007). The investigation strategy required the nature and extent of contamination associated with each CAS to be established. The following sections describe the specific investigation activities that took place at CAU 234.

\section{A.2.2.1 Radiological Surveys}

Radiological surveys (i.e., scanning, static, and swipe collection) were performed at all CASs during the CAI. Radiological surveys were performed to identify the presence, nature, and extent of radiological contaminants at activities statistically distinguishable from background activities (more than two times background levels). The radiological surveys were conducted using a handheld plastic scintillation detector in conjunction with a GPS receiver and datalogger.

\section{A.2.2.2 Field Screening}

Field-screening activities were conducted for alpha and beta/gamma radiation, and gamma-emitting radionuclides as specified in the CAU 234 CAIP (NNSA/NSO, 2007). Site-specific FSLs for alpha and beta/gamma radiation were defined as the mean background activity level plus two times the standard deviation of readings from 10 background locations selected near each CAS. The radiation FSLs are instrument-specific and were established for each instrument and CAS before use.

The CAS-specific sections of this document identify the CASs where field screening was conducted and how the FSLs were used to aid in the selection of sample locations. Field-screening results are recorded on SCLs that are retained in project files. 


\section{A.2.2.3 Surface and Subsurface Soil Sampling}

Soil samples were collected using "scoop and trowel" (surface hand-grab sampling) and hand auger procedures. All sample locations were initially field screened for alpha and beta/gamma radiation before the start of sampling. Additional screening was conducted during sample collection to both guide the investigation and serve as a health and safety control to protect the sampling team. Labeled sample containers were filled according to the following sequence: volatile organic compound (VOC) sample containers were filled with soil directly from the sample location. Additional soil was transferred into an aluminum pan, homogenized, and field screened for alpha and beta/gamma radiation. All remaining sample containers were then filled. Excess soil was returned to its original location and the sample containers appropriately disposed (based on field-screening and/or analytical results).

Surface soil samples were collected from 0.0 to $0.5 \mathrm{ft}$ bgs at biased locations, except where refusal (a physical anomaly that does not allow further penetration below ground) was encountered due to shallow underlying bedrock. Subsurface soil samples were collected as a continuation at surface soil sample locations except where refusal was encountered. The SCLs describe when refusal conditions were encountered.

\section{A.2.2.4 Waste Characterization and Potential Source Material Sampling}

Characterization of CAS-specific components, objects, materials, and waste was performed to support disposal of these potential remediation wastes and to determine whether any materials located within the specific feature could be potential source material (PSM). Investigation methods included visual inspection, radiological surveys, and direct sampling of the contents of each feature, where available.

Samples were analyzed in accordance with the CAU 234 CAIP (NNSA/NSO, 2007). The specific analyses for each CAS are listed in CAS-specific sections, and the analytical results are compared to the federal limits for hazardous waste, landfill acceptance criteria, and the limits in the NTS performance objective criteria (POC) (BN, 1995). The POC limits have been established for NTS hazardous waste generators to ensure that all hazardous waste being shipped off site contains no "added radioactivity." 
Specific waste characterization sampling and analysis was conducted on the following potential waste streams:

- The investigation-derived waste (IDW) rinsate drum generated at CAS 03-09-02

- Debris (see Table A.8-1)

Potential source material sampling and evaluation was performed on the following media:

- Liquids contained within the cellar at CAS 12-30-14.

- Sediment within the cellar at CAS 12-30-14.

\section{A.2.3 Laboratory Analytical Information}

Radiological and chemical analyses were performed by Paragon Analytics, Inc., of Fort Collins, Colorado. The analytical suites and laboratory analytical methods used to analyze investigation samples are listed in Table A.2-2. Analytical results are reported in this appendix if they were detected above the MDCs. The complete laboratory data packages are available in the project files. Validated analytical data for CAU 234 investigation samples have been compiled and evaluated to confirm the presence of contamination and define the extent of contamination, if present. The analytical results for each CAS are presented in Sections A.3.0 through A.7.0.

Table A.2-2

Laboratory Analytical Parameters and Methods, CAU 234 Investigation Samples ${ }^{\mathrm{a}}$ (Page 1 of 2)

\begin{tabular}{|c|c|}
\hline Analytical Parameter & Analytical Method ${ }^{\mathrm{b}}$ \\
\hline Volatile Organic Compounds & EPA SW-846 8260B ${ }^{c}$ \\
\hline Semivolatile Organic Compounds & EPA SW-846 $8270 C^{c}$ \\
\hline $\begin{array}{l}\text { Total Petroleum Hydrocarbons- } \\
\text { Diesel Range Organics }\end{array}$ & EPA SW-846 8015B \\
\hline RCRA Metals ${ }^{d}$ & EPA SW-846 6010B/7470A/7471 $\mathrm{A}^{\mathrm{C}}$ \\
\hline Polychlorinated Biphenyls & EPA SW-846 $8082^{c}$ \\
\hline Gamma Spectroscopy & DOE EML HASL $300^{\circ}$ Approved Laboratory SOPs ${ }^{\dagger}$ \\
\hline Isotopic Uranium & $\begin{array}{l}\text { DOE EML HASL- } 300^{e} \text { U-02-RC Modified, Approved Laboratory } \\
\text { SOPs }{ }^{\dagger}\end{array}$ \\
\hline Isotopic Plutonium & $\begin{array}{l}\text { DOE EML HASL-300 PU-02-RC/PU-10-RC Modified, Approved } \\
\text { Laboratory SOPs }{ }^{\dagger}\end{array}$ \\
\hline
\end{tabular}


Table A.2-2

Laboratory Analytical Parameters and Methods, CAU 234 Investigation Samples ${ }^{a}$ (Page 2 of 2)

\begin{tabular}{|c|c|}
\hline Analytical Parameter & Analytical Method ${ }^{\mathrm{b}}$ \\
\hline Strontium-90 & EPA $905.0^{9}$ Modified, Approved Laboratory SOPs ${ }^{\dagger}$ \\
\hline Gross Alpha/Beta & EPA $900.0^{9}$ Modified, Approved Laboratory SOPs ${ }^{\dagger}$ \\
\hline Tritium & EPA $906.0^{9}$ Modified, Approved Laboratory SOPs ${ }^{\dagger}$ \\
\hline
\end{tabular}

\footnotetext{
anvestigation samples include both environmental and waste characterization samples and associated quality control samples. ${ }^{\mathrm{b}}$ The most current EPA, DOE, ASTM, or NIOSH or equivalent accepted analytical method may be used.

${ }^{\mathrm{c}}$ Test Methods for Evaluating Solid Waste, Physical/Chemical Methods, $3{ }^{\text {rd }}$ edition, Parts 1-4, SW-846 CD-ROM (EPA, 1996).

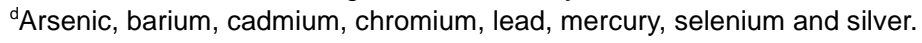

${ }^{\mathrm{e}}$ The Procedures Manual of the Environmental Measurements Laboratory, HASL-300 (DOE, 1997).

'Laboratory Standard Operating Procedures approved by SNJV in accordance with industry standards and the SNJV Model Statement of Work requirements (SNJV, 2006).

${ }^{9}$ Prescribed Procedures for Measurement of Radioactivity in Drinking Water (EMSL/ORD, 1980).
}

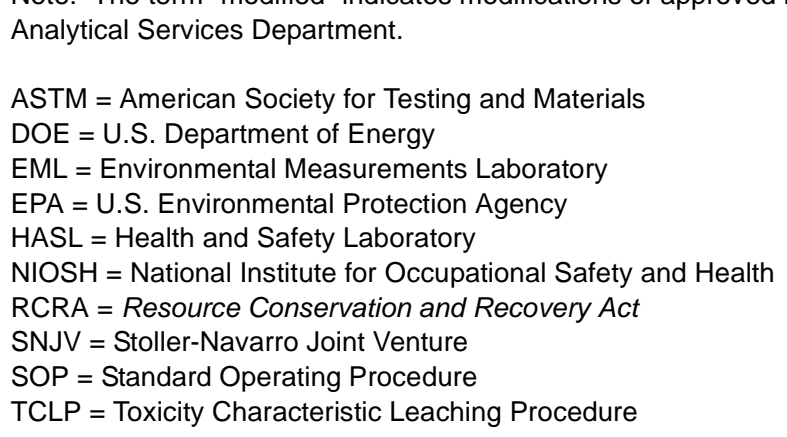

The analytical parameters are CAS-specific and were selected through the application of site process knowledge as described in the CAIP DQOs (NNSA/NSO, 2007).

\section{A.2.4 Comparison to Action Levels}

A COC is defined as any contaminant present in environmental media exceeding a FAL. A COC may also be defined as a contaminant that, in combination with other like contaminants, is determined to jointly pose an unacceptable risk based on a multiple constituent analysis (NNSA/NSO, 2006b). Multiple constituent analyses are presented in Appendix D.

If COCs are present, corrective action must be considered for the CAS. The FALs for the CAU 234 investigation are defined for each CAS in Section 2.3.1. 
The evaluation of the need for corrective action will include the potential for wastes that are present at a site to cause the future contamination of site environmental media if the wastes were to be released. To evaluate the potential for cellar contents of CAS 12-30-14 to result in the introduction of a COC to the surrounding environmental media, the following conservative assumptions were made:

- The cellar containment would fail at some point, and the contents would be released to the surrounding media.

- The resulting concentration of contaminants in the surrounding media would be equal to the concentration of contaminants in the cellar.

- Any liquid contaminant in the cellar exceeding the RCRA TC concentration can result in a COC's introduction to the surrounding media.

- Sludge possibly containing a contaminant exceeding an equivalent FAL concentration would be considered to be PSM requiring a corrective action.

- Cellar liquids with possible contaminant concentrations exceeding an equivalent TC action level would be considered to be PSM requiring a corrective action. 


\section{A.3.0 Corrective Action Site 02-09-48, Area 2 Mud Plant \#1}

Corrective Action Site 02-09-48 is located at the Area 2 Mud Plant of the NTS (Figure A.3-1). The Area 2 Mud Plant manufactured drilling mud for use in drilling operations at the NTS. The CAS is a concrete-lined sump used for storage of drilling muds until they were needed for drilling operations. A rusted, 55-gal drum was located on the surface of the drilling mud within the sump. Additional detail is provided in the CAIP (NNSA/NSO, 2007).

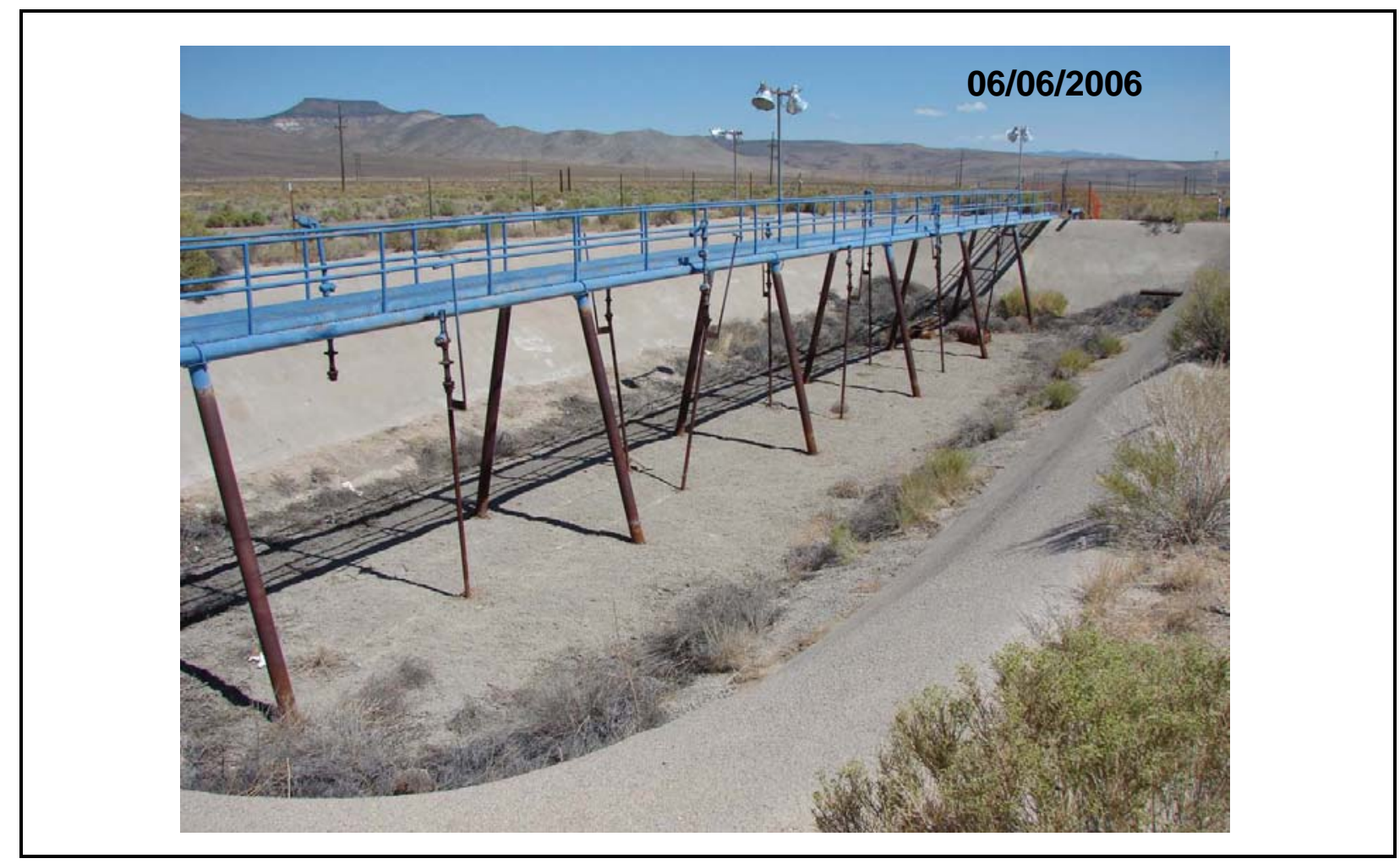

Figure A.3-1

Corrective Action Site 02-09-48

\section{A.3.1 Corrective Action Investigation}

A total of five characterization samples (including one FD) were collected during investigation activities at CAS 02-09-48. The sample IDs, locations, types, and analyses are listed in Table A.3-1. The specific CAI activities conducted to satisfy the CAIP requirements at this CAS (NNSA/NSO, 2007) are described in the following sections. 
Table A.3-1

Samples Collected at CAS 02-09-48, Area 2 Mud Plant \#1

\begin{tabular}{||c|c|c|c|c|c||}
\hline $\begin{array}{c}\text { Sample } \\
\text { Location }\end{array}$ & $\begin{array}{c}\text { Sample } \\
\text { Number }\end{array}$ & $\begin{array}{c}\text { Depth } \\
\text { (ft bgs) }\end{array}$ & Matrix & Purpose & Analyses \\
\hline \hline \multirow{3}{*}{ A01 } & $234 \mathrm{~A} 001$ & $0.0-0.5$ & Soil & Environmental & Set 1 \\
\cline { 2 - 6 } & $234 \mathrm{~A} 002$ & $0.0-0.5$ & Soil & Field Duplicate of 234A001 & Set 1 \\
\cline { 2 - 6 } & $234 \mathrm{~A} 003$ & $0.5-1.0$ & Soil & Environmental & Set 1 \\
\hline \multirow{2}{*}{ A02 } & $234 \mathrm{~A} 004$ & $0.0-0.5$ & Soil & Environmental & Set 1 \\
\cline { 2 - 6 } & $234 \mathrm{~A} 005$ & $0.5-1.0$ & Soil & Environmental & Set 1 \\
\hline N/A & $234 \mathrm{~A} 301$ & N/A & Water & Trip Blank & VOCs only \\
\hline N/A & $234 \mathrm{~A} 302$ & N/A & Water & Trip Blank & VOCs only \\
\hline N/A & $234 \mathrm{~A} 303$ & N/A & Water & Field Blank & Set 1 \\
\hline
\end{tabular}

Set 1 = VOCs, SVOCs, RCRA Metals, TPH-DRO, PCBs, Gamma Spectroscopy, Isotopic Uranium, Isotopic Plutonium, Strontium-90

bgs $=$ Below ground surface

$\mathrm{DRO}=$ Diesel-range organics

$\mathrm{ft}=$ Foot

$\mathrm{N} / \mathrm{A}=$ Not applicable

$\mathrm{PCB}=$ Polychlorinated biphenyl
RCRA $=$ Resource Conservation and Recovery Act

SVOC $=$ Semivolatile organic compound

$\mathrm{TPH}=$ Total petroleum hydrocarbons

$\mathrm{VOC}=$ Volatile organic compound

\section{A.3.1.1 Field Screening}

Investigation samples were field screened for alpha and beta/gamma radiation. Gross alpha radiation FSLs were not exceeded in any of the samples. Beta/gamma radiation FSLs were not exceeded in any of the samples. Therefore, no additional biasing factors were identified, and no additional samples were collected.

\section{A.3.1.2 Radiological Surveys}

As presented in the CAIP (NNSA/NSO, 2007), a radiological walkover survey was conducted on May 23, 2006, on the mud sump. The survey did not identify radiation that was significantly different from background. Therefore, no additional biasing factors were identified, and no additional samples were collected. 


\section{A.3.1.3 Visual Inspections}

One feature associated with the drilling mud sump other than the drilling mud itself was identified within the CAS. This feature consisted of a rusting, 55-gal drum. The drum was empty, so a sample of its contents was not collected. Initial inspection indicated that the drum was rusted and dry, and that the bungs had been removed.

Inspections of the drilling mud sump did not identify additional sample locations based on biasing factors (i.e., staining).

\section{A.3.1.4 Sample Collection}

Environmental sampling activities included the collection of biased surface and subsurface soil samples surrounding the rusted, 55-gal drum (Figure A.3-2).

\section{A.3.1.5 Deviations}

Investigation samples were collected as outlined in the CAU 234 CAIP (NNSA/NSO, 2007) and submitted for laboratory analysis with no deviations from the planned sample locations.

\section{A.3.2 Investigation Results}

The following sections provide analytical results from the samples collected to complete investigation activities as outlined in the CAIP (NNSA/NSO, 2007). Investigation samples were analyzed for the CAIP-specified COPCs, which included VOCs, semivolatile organic compounds (SVOCs), TPH-diesel-range organics (DRO), RCRA metals, gamma-emitting radionuclides, isotopic uranium $(\mathrm{U})$, isotopic plutonium $(\mathrm{Pu})$, and strontium $(\mathrm{Sr})-90$. The polychlorinated biphenyls (PCBs) are added parameters because these contaminants are a common concern at the NTS. The analytical parameters and laboratory methods used to analyze the investigation samples are listed in Table A.2-2. Table A.3-1 lists the sample-specific analytical suite for CAS 02-09-48.

Analytical results from the soil samples with concentrations exceeding MDCs are summarized in the following sections. An evaluation was conducted on all contaminants detected above MDCs by comparing individual concentration or activity results against the FALs. Establishment of the FALs is presented in Appendix C. 


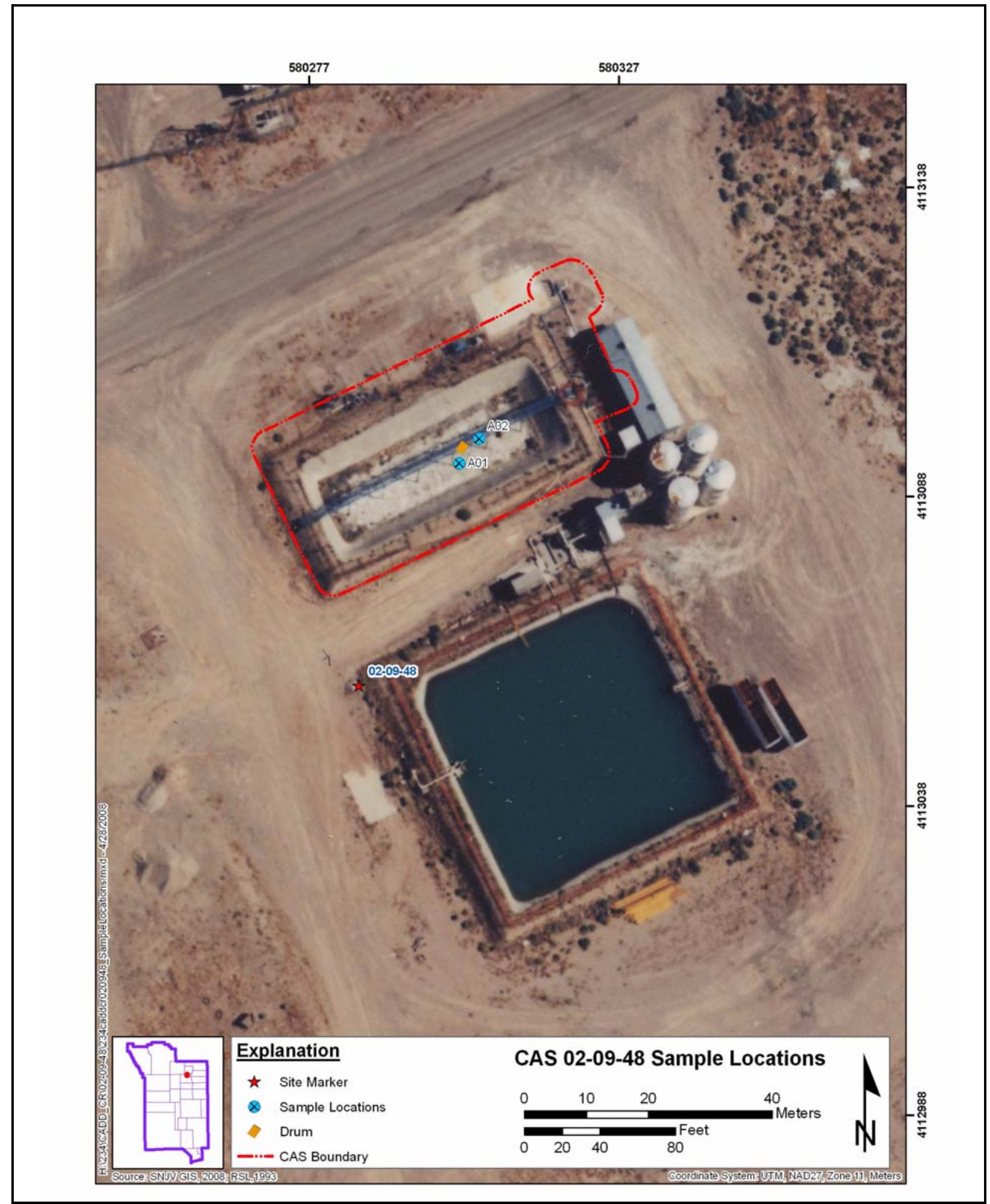

Figure A.3-2

Sample Locations for CAS 02-09-48 


\section{A.3.2.1 Volatile Organic Compounds}

No VOC analytical results for environmental samples collected at this CAS were detected above their respective MDCs. Therefore, the FALs were established at the corresponding PAL concentrations.

\section{A.3.2.2 Semivolatile Organic Compounds}

The SVOCs analytical results for environmental samples collected at this CAS detected above their respective MDCs are shown in Table A.3-2. None of the results exceeded their respective PALs. Therefore, the FALs were established at the corresponding PAL concentrations.

Table A.3-2

Soil Sample Results of SVOCs Detected above Minimum Detectable Concentrations at CAS 02-09-48, Area 2 Mud Plant \#1

\begin{tabular}{|c|c|c|c|c|c|c|c|c|}
\hline \multirow[b]{2}{*}{$\begin{array}{c}\text { Sample } \\
\text { Location }\end{array}$} & \multirow[b]{2}{*}{$\begin{array}{l}\text { Sample } \\
\text { Number }\end{array}$} & \multirow[b]{2}{*}{$\begin{array}{l}\text { Depth } \\
\text { (ft bgs) }\end{array}$} & \multicolumn{6}{|c|}{ Contaminants of Potential Concern $(\mathrm{mg} / \mathrm{kg})$} \\
\hline & & & 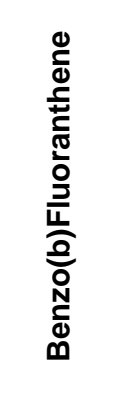 & 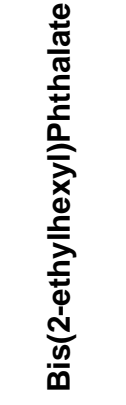 & 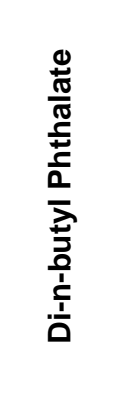 & 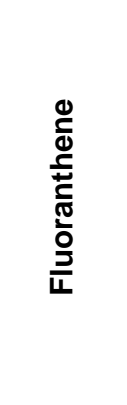 & 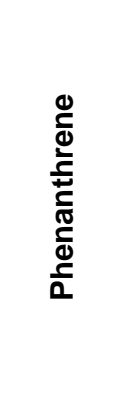 & 离 \\
\hline \multicolumn{3}{|c|}{ Final Action Levels ${ }^{a}$} & 2.1 & 120 & 62,000 & 22,000 & 100,000 & 29,000 \\
\hline \multirow{2}{*}{ A01 } & $234 \mathrm{~A} 001$ & $0.0-0.5$ & $0.16(\mathrm{~J})$ & -- & $0.27(\mathrm{~J})$ & $0.25(\mathrm{~J})$ & $0.16(\mathrm{~J})$ & $0.19(\mathrm{~J})$ \\
\hline & 234A002 & $0.0-0.5$ & $0.18(\mathrm{~J})$ & -- & $0.34(\mathrm{~J})$ & $0.28(\mathrm{~J})$ & $0.2(\mathrm{~J})$ & $0.2(\mathrm{~J})$ \\
\hline \multirow{2}{*}{ A02 } & 234A004 & $0.0-0.5$ & $0.13(\mathrm{~J})$ & -- & $0.15(\mathrm{~J})$ & $0.13(\mathrm{~J})$ & -- & -- \\
\hline & $234 A 005$ & $0.5-1.0$ & -- & $0.16(\mathrm{~J})$ & -- & -- & -- & -- \\
\hline
\end{tabular}

aBased on U.S. Environmental Protection Agency, Region 9 Preliminary Remediation Goals (PRGs) (EPA, 2004).

bgs $=$ Below ground surface

$\mathrm{ft}=$ Foot

$\mathrm{mg} / \mathrm{kg}=$ Milligrams per kilogram

$\mathrm{J}=$ Estimated value

$--=$ Not detected above minimum detectable concentrations 


\section{A.3.2.3 Total Petroleum Hydrocarbons}

Analytical results for soil samples collected at this CAS indicated that TPH-DRO was not detected above its respective MDC. Therefore, the FAL was established at the PAL concentration.

\section{A.3.2.4 RCRA Metals}

The RCRA metals analytical results for environmental samples collected at this CAS that were detected above MDCs are presented in Table A.3-3. No metals were detected at concentrations exceeding their PALs. Therefore, the FALs were established at the corresponding PAL concentrations.

Table A.3-3

Soil Sample Results for RCRA Metals Detected above Minimum Detectable Concentrations at CAS 02-09-48, Area 2 Mud Plant \#1

\begin{tabular}{||c|c|c|c|c|c|c|c||}
\hline \multirow{2}{*}{$\begin{array}{c}\text { Sample } \\
\text { Location }\end{array}$} & \multirow{2}{*}{$\begin{array}{c}\text { Sample } \\
\text { Number }\end{array}$} & \multirow{2}{*}{$\begin{array}{c}\text { Depth } \\
\text { (ft bgs) }\end{array}$} & \multicolumn{5}{|c||}{ Contaminants of Potential Concern (mg/kg) } \\
\cline { 3 - 8 } & & & Arsenic & Barium & Cadmium & Chromium & Lead \\
\hline \multicolumn{3}{|c|}{ Final Action Levels } & $\mathbf{2 3}^{\mathrm{a}}$ & $\mathbf{6 7 , 0 0 0 ^ { \mathrm { b } }}$ & $\mathbf{4 5 0 ^ { \mathrm { b } }}$ & $\mathbf{4 5 0 ^ { \mathrm { b } }}$ & $\mathbf{8 0 0}^{\mathrm{b}}$ \\
\hline \hline \multirow{3}{*}{$\mathrm{A} 01$} & $234 \mathrm{~A} 001$ & $0.0-0.5$ & 2.3 & 100 & 0.65 & 3.8 & 29 \\
\cline { 2 - 9 } & $234 \mathrm{~A} 002$ & $0.0-0.5$ & 2.8 & 97 & 0.55 & 3.5 & 27 \\
\cline { 2 - 9 } & $234 \mathrm{~A} 003$ & $0.5-1.0$ & 1.6 & 91 & 0.28 & -- & 29 \\
\hline \multirow{2}{*}{ A02 } & $234 \mathrm{~A} 004$ & $0.0-0.5$ & 2.3 & 85 & 0.31 & 2.2 & 29 \\
\cline { 2 - 9 } & $234 \mathrm{~A} 005$ & $0.5-1.0$ & 1.7 & 58 & 0.19 & -- & 24 \\
\hline
\end{tabular}

aBased on the background concentrations for metals. Background is considered the mean plus two times the standard deviation for sediment samples collected by the Nevada Bureau of Mines and Geology throughout the Nevada Test and Training Range (NBMG, 1998; Moore, 1999).

${ }^{\mathrm{b} B a s e d}$ on U.S. Environmental Protection Agency, Region 9 Preliminary Remediation Goals (PRGs) (EPA, 2004).

bgs $=$ Below ground surface

$\mathrm{ft}=$ Foot

$\mathrm{mg} / \mathrm{kg}=$ Milligrams per kilogram

$--=$ Not detected above minimum detectable concentrations 


\section{A.3.2.5 Polychlorinated Biphenyls}

Analytical results for soil samples collected at this CAS indicated that there were no PCBs detected above their respective MDCs. Therefore, the FALs were established at the corresponding PAL concentrations.

\section{A.3.2.6 Gamma-Emitting Radionuclides}

Gamma-emitting radionuclides analytical results for environmental samples collected at this CAS that were detected above MDCs are presented in Table A.3-4. None of the analytical results were above the respective PALs for any of the analytes. Therefore, the FALs were established at the corresponding PAL concentrations.

Table A.3-4

Soil Sample Results for Gamma-Emitting Radionuclides Detected above Minimum Detectable Concentrations at CAS 02-09-48, Area 2 Mud Plant \#1

\begin{tabular}{|c|c|c|c|c|c|c|c|c|c|c|c|}
\hline \multirow{2}{*}{$\begin{array}{l}\text { Sample } \\
\text { Location }\end{array}$} & \multirow{2}{*}{$\begin{array}{l}\text { Sample } \\
\text { Number }\end{array}$} & \multirow{3}{*}{$\begin{array}{l}\text { Depth } \\
\text { (ft bgs) }\end{array}$} & \multicolumn{9}{|c|}{ Contaminants of Potential Concern (pCi/g) } \\
\hline & & & \multicolumn{2}{|c|}{ Actinium-228 } & \multicolumn{2}{|c|}{ Lead-212 } & \multicolumn{2}{|c|}{ Lead-214 } & \multirow{2}{*}{\begin{tabular}{|c|} 
Thorium-234 \\
$105^{\mathrm{b}}$
\end{tabular}} & \multicolumn{2}{|c|}{ Thallium-208 } \\
\hline \multicolumn{2}{|c|}{ Final Action Levels } & & $5^{a}$ & $15^{a}$ & $5^{a}$ & $15^{a}$ & $5^{a}$ & $15^{a}$ & & $5^{a}$ & $15^{a}$ \\
\hline \multirow{3}{*}{$\mathrm{A} 01$} & $234 \mathrm{~A} 001$ & $0.0-0.5$ & 2.85 & -- & $3.11(\mathrm{~J})$ & -- & $2.59(\mathrm{~J})$ & -- & $4.1(\mathrm{~J})$ & 0.84 & -- \\
\hline & $234 \mathrm{~A} 002$ & $0.0-0.5$ & 2.57 & -- & $2.99(\mathrm{~J})$ & -- & $2.76(\mathrm{~J})$ & -- & $4.6(\mathrm{~J})$ & 0.87 & -- \\
\hline & $234 \mathrm{~A} 003$ & $0.5-1.0$ & -- & 3.29 & -- & $4.12(\mathrm{~J})$ & -- & $3.1(\mathrm{~J})$ & $4.4(\mathrm{~J})$ & -- & 1.21 \\
\hline \multirow{2}{*}{$\mathrm{A} 02$} & $234 \mathrm{~A} 004$ & $0.0-0.5$ & 3.31 & -- & $3.18(\mathrm{~J})$ & -- & $2.93(\mathrm{~J})$ & -- & $3.8(\mathrm{~J})$ & 1.22 & -- \\
\hline & $234 \mathrm{~A} 005$ & $0.5-1.0$ & -- & 3.72 & -- & $3.63(\mathrm{~J})$ & -- & $3.36(\mathrm{~J})$ & $4.6(\mathrm{~J})$ & -- & 1.09 \\
\hline
\end{tabular}

Taken from the generic guidelines for residual concentrations of actinium-228, bismuth-214, lead-212, lead-214, thallium-208, and thorium-232, as found in Chapter IV of DOE Order 5400.5, Change 2, "Radiation Protection of the Public and Environment." (DOE, 1993). The PALs for these isotopes are specified as $5 \mathrm{pCi} / \mathrm{g}$ averaged over the first $15 \mathrm{~cm}$ of soil and $15 \mathrm{pCi} / \mathrm{g}$ for deeper soils (DOE, 1993). For purposes of this document, $15 \mathrm{~cm}$ is assumed to be equivalent to $0.5 \mathrm{ft}$ ( 6 inches); therefore, $5 \mathrm{pCi} / \mathrm{g}$ represents the PALs for these radionuclides in the surface soil ( 0 to $0.5 \mathrm{ft}$ depth).

${ }^{\mathrm{b}}$ Taken from the construction, commercial, industrial land use scenario in Table 2.1 of the NCRP Report No. 129, Recommended Screening Limits for Contaminated Surface Soil and Review Factors Relevant to Site-Specific Studies (NCRP, 1999). The values provided in this source document were scaled to a 25-millirem-per-year dose.

bgs $=$ Below ground surface

$\mathrm{cm}=$ Centimeter

DOE $=$ U.S. Department of Energy

$\mathrm{ft}=$ Foot

NCRP $=$ National Council on Radiation Protection and Measurements

$\mathrm{PAL}=$ Preliminary action level

$\mathrm{pCi} / \mathrm{g}=$ Picocuries per gram

$\mathrm{J}=$ Estimated value

-- = Not detected above minimum detectable concentrations 


\section{A.3.2.7 Plutonium, Strontium-90, and Uranium Isotopes}

Isotopic $\mathrm{Pu}$ and isotopic $\mathrm{U}$ analytical results for environmental samples collected at this CAS that were detected above MDCs are presented in Table A.3-5. No Sr-90 was identified above its MDC in any of the samples analyzed. None of the $\mathrm{Pu}, \mathrm{U}$, or $\mathrm{Sr}-90$ isotope results were above their respective PALs in any of the samples analyzed. Therefore, the FALs were established at the corresponding PAL concentrations.

Table A.3-5

Soil Sample Results for Isotopes Detected above Minimum Detectable Concentrations at CAS 02-09-48, Area 2 Mud Plant \#1

\begin{tabular}{|c|c|c|c|c|c|c|c|}
\hline \multirow{2}{*}{$\begin{array}{l}\text { Sample } \\
\text { Location }\end{array}$} & \multirow{2}{*}{$\begin{array}{l}\text { Sample } \\
\text { Number }\end{array}$} & \multirow{2}{*}{$\begin{array}{l}\text { Depth } \\
\text { (ft bgs) }\end{array}$} & \multicolumn{5}{|c|}{ Contaminants of Potential Concern (pCi/g) } \\
\hline & & & Plutonium-238 & Plutonium-239/240 & Uranium-234 & Uranium-235 & Uranium-238 \\
\hline \multicolumn{3}{|c|}{ Final Action Levels ${ }^{a}$} & 13 & 12.7 & 143 & 17.6 & 105 \\
\hline \multirow{3}{*}{ A01 } & 234A001 & $0.0-0.5$ & 0.093 & 0.35 & 2.5 & 0.113 & 2.37 \\
\hline & 234A002 & $0.0-0.5$ & -- & 0.128 & 2.2 & 0.16 & 2.33 \\
\hline & 234A003 & $0.5-1.0$ & -- & -- & 2.59 & 0.147 & 2.48 \\
\hline \multirow{2}{*}{$\mathrm{A} 02$} & 234A004 & $0.0-0.5$ & -- & 0.211 & 2.53 & 0.136 & 2.55 \\
\hline & $234 \mathrm{~A} 005$ & $0.5-1.0$ & -- & 0.036 & 2.36 & 0.145 & 2.66 \\
\hline
\end{tabular}

aTaken from the construction, commercial, industrial land use scenario in Table 2.1 of the NCRP Report No. 129, Recommended Screening Limits for Contaminated Surface Soil and Review Factors Relevant to Site-Specific Studies (NCRP, 1999). The values provided in this source document were scaled to a 25-millirem-per-year dose.

bgs $=$ Below ground surface

$\mathrm{ft}=$ Foot

NCRP $=$ National Council on Radiation Protection and Measurements

$\mathrm{pCi} / \mathrm{g}=$ Picocuries per gram

$--=$ Not detected above minimum detectable concentrations

\section{A.3.3 Nature and Extent of Contamination}

Based on the analytical results for soil samples collected within CAS 02-09-48, none of the samples exceeded the FALs for any of the analytes identified above their MDCs. Therefore, no COCs are present at this CAS.

\section{A.3.4 Revised Conceptual Site Model}

The CAIP requirements (NNSA/NSO, 2007) were met at this CAS, and no revisions were necessary to the CSM. 


\section{A.4.0 Corrective Action Site 03-09-02, Mud Dump Trenches}

Corrective Action Site 03-09-02 is located in the northwest corner of Area 3 of the NTS. This CAS consists of two distinct drilling mud suction/return pits used for the construction of the U-3kz emplacement hole. The two distinct systems are oriented north/south of each other and therefore are referred to as the northern footprint and the southern footprint of the CAS (Figures A.4-1 and A.4-2). The northern footprint also contains an area identified as "possible reserve suction pit," but it appears unlikely it was ever used as such. The southern footprint suction/return pits reside in a large area that was possibly used as a borrow pit before the dual pit construction used for drilling the emplacement hole. Dried drilling mud is visible in the northern footprint return pit and both the suction and return pits in the southern footprints. The northern footprint suction pit is filled with tumbleweeds. After removal of the tumbleweeds, it was discovered that a discarded length of blue pipe was located in the southern berm of the suction pit. Sampling occurred at the pipe as well as the points of lowest elevation within the two northern footprint suction/return pits. An additional sample location was identified within the "possible reserve suction pit" in the northern footprint at the location of lowest elevation.

\section{A.4.1 Corrective Action Investigation}

A total of 14 characterization samples (including one FD) were collected during investigation activities at CAS 03-09-02. The sample IDs, locations, types, and analyses are listed in Table A.4-1. The specific CAI activities conducted to satisfy the CAIP requirements at this CAS (NNSA/NSO, 2007) are described in the following sections.

\section{A.4.1.1 Field Screening}

Soil samples were field screened for alpha and beta/gamma radiation as specified in the CAU 234 CAIP (NNSA/NSO, 2007). The FSRs were compared to FSLs to guide subsequent sampling decisions where appropriate. Alpha and beta/gamma radiation FSLs were not exceeded during sampling activities. As a result, no additional samples were collected. 


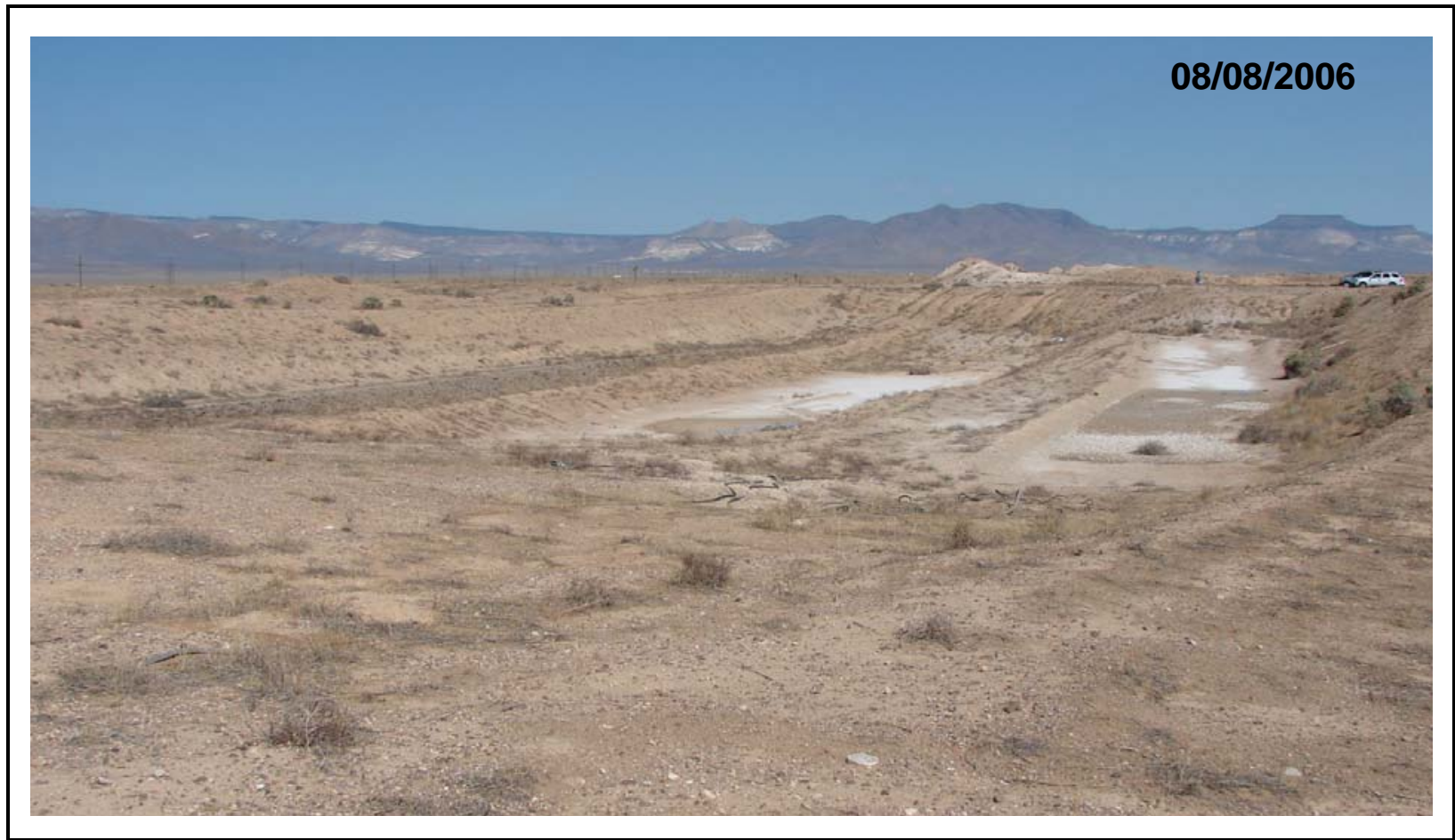

Figure A.4-1

\section{Mud Trenches in Southern Footprint}

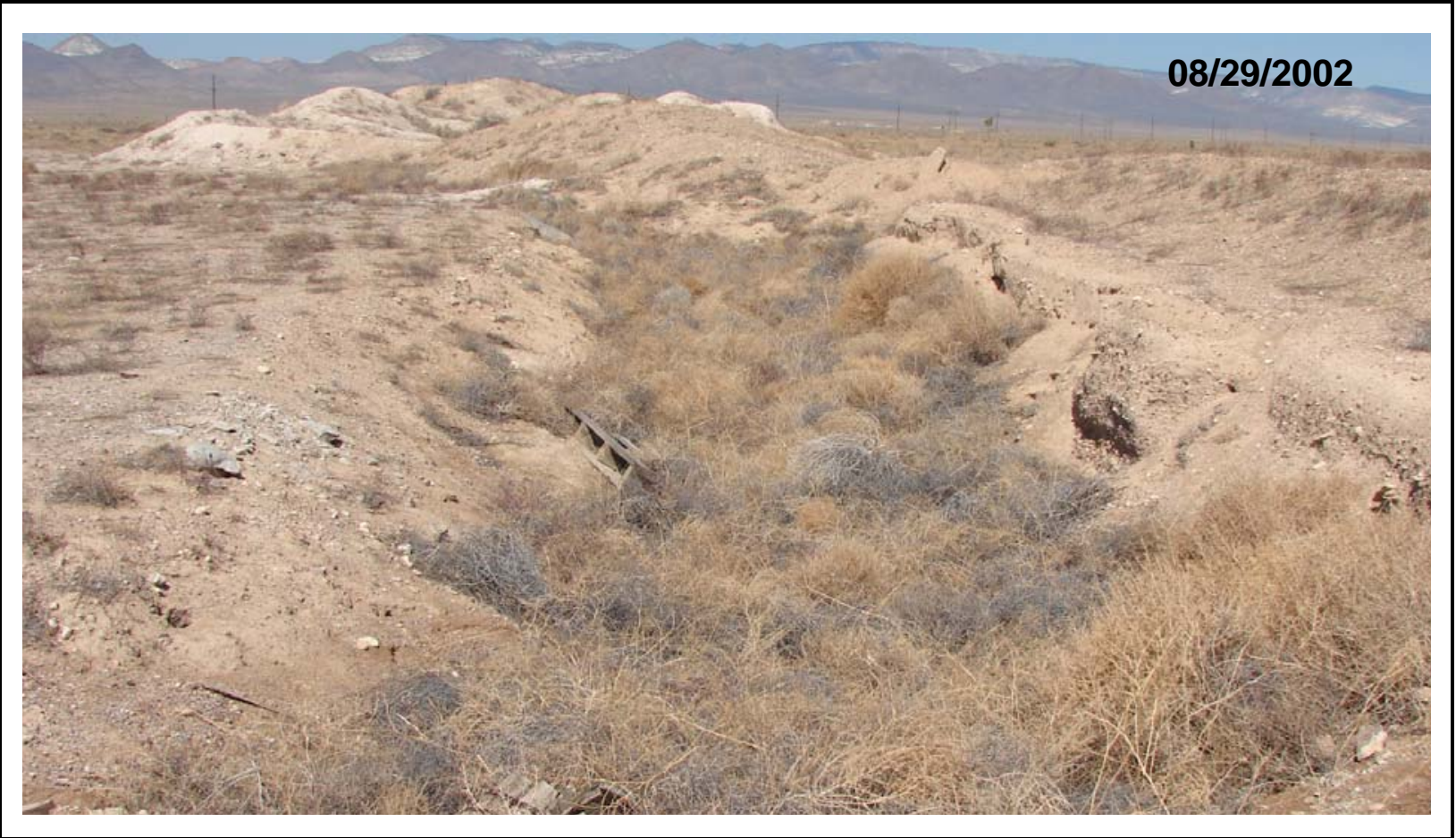

Figure A.4-2

Suction Pit in Northern Footprint 
Table A.4-1

Samples Collected at CAS 03-09-02, Mud Dump Trenches

\begin{tabular}{|c|c|c|c|c|c|}
\hline $\begin{array}{l}\text { Sample } \\
\text { Location }\end{array}$ & $\begin{array}{l}\text { Sample } \\
\text { Number }\end{array}$ & $\begin{array}{c}\text { Depth } \\
\text { (ft bgs) }\end{array}$ & Matrix & Purpose & Analyses \\
\hline \multirow{3}{*}{ B01 } & 234B001 & $0.0-0.5$ & Soil & Environmental & Set 1 \\
\hline & 234B002 & $0.0-0.5$ & Soil & Field Duplicate of 234B001 & Set 1 \\
\hline & 234B005 & $1.5-2.0$ & Soil & Environmental & Set 1 \\
\hline \multirow{2}{*}{ B02 } & 234B003 & $0.0-0.5$ & Soil & Environmental & Set 1 \\
\hline & 234B004 & $1.5-2.0$ & Soil & Environmental & Set 1 \\
\hline \multirow{2}{*}{ B03 } & 234B006 & $0.0-0.5$ & Soil & Environmental & Set 1 \\
\hline & 234B007 & $1.5-2.0$ & Soil & Environmental & Set 1 \\
\hline \multirow{2}{*}{ B04 } & 234B008 & $0.0-0.5$ & Soil & Environmental & Set 1 \\
\hline & 234B009 & $1.5-2.0$ & Soil & Environmental & Set 1 \\
\hline \multirow{2}{*}{ B05 } & 234B010 & $0.0-0.5$ & Soil & Environmental & Set 1 \\
\hline & 234B011 & $1.5-2.0$ & Soil & Environmental & Set 1 \\
\hline \multirow{2}{*}{ B06 } & 234B012 & $0.0-0.5$ & Soil & Environmental & Set 1 \\
\hline & 234B013 & $1.5-2.0$ & Soil & Environmental & Set 1 \\
\hline B07 & 234B014 & $0.0-0.5$ & Soil & Environmental & Set 1 \\
\hline $\mathrm{N} / \mathrm{A}$ & 234B301 & $\mathrm{N} / \mathrm{A}$ & Water & Trip Blank & VOCs only \\
\hline $\mathrm{N} / \mathrm{A}$ & 234B302 & $\mathrm{N} / \mathrm{A}$ & Water & Equipment Rinsate Blank & Set 1 \\
\hline $\mathrm{N} / \mathrm{A}$ & 234B303 & $\mathrm{N} / \mathrm{A}$ & Water & Field Blank & Set 1 \\
\hline $\mathrm{N} / \mathrm{A}$ & 234B304 & $\mathrm{N} / \mathrm{A}$ & Water & Trip Blank & VOCs only \\
\hline $\mathrm{N} / \mathrm{A}$ & 234B501 & $\mathrm{N} / \mathrm{A}$ & Liquid & Waste Management & Set 2 \\
\hline
\end{tabular}

Set 1 = VOCs, SVOCs, RCRA Metals, TPH-DRO, PCBs, Gamma Spectroscopy, Isotopic Uranium, Isotopic Plutonium, Strontium-90

Set 2 = VOCs, SVOCs, RCRA Metals, TPH-DRO, PCBs, Gamma Spectroscopy, Isotopic Uranium, Isotopic Plutonium, Strontium-90, Grass Alpha/Beta, Tritium

bgs = Below ground surface

$\mathrm{DRO}=$ Diesel-range organics

$\mathrm{ft}=$ Foot

$\mathrm{N} / \mathrm{A}=$ Not applicable

$\mathrm{PCB}=$ Polychlorinated biphenyl
RCRA $=$ Resource Conservation and Recovery Act

SVOC = Semivolatile organic compound

$\mathrm{TPH}=$ Total petroleum hydrocarbons

VOC $=$ Volatile organic compound 


\section{A.4.1.2 Radiological Surveys}

A radiological walkover survey was conducted on October 25, 2007, over the sampling areas of interest (i.e., mud pits, suction pits). This is presented in Figure A.4-3. The survey did not identify radiation that was distinguishable from background. As a result, no additional samples were collected.

\section{A.4.1.3 Visual Inspections}

The site was visually inspected for potential sources of contamination before sample collection. A length of blue piping was identified lying in the suction pit in the southern berm after all the tumbleweeds had been cleared out. No other points of interest were identified.

A small puddle of water was identified at the selected sample location within the mud trench (in the southern footprint). Sample location B02 was selected at the outer edge of the puddle of water, but was identified as a point that was not the lowest in the trench. On January 23, 2008, a sample was collected beneath the puddle of water at the location identified as the lowest point in the trench, in accordance with the requirements of the CAIP (NNSA/NSO, 2007).

\section{A.4.1.4 Sample Collection}

Decision I environmental sampling activities included the collection of biased surface and subsurface soil samples at the low elevations in each of the pits that handled drilling mud from the U-3kz emplacement hole project, as well as beneath a piece of blue pipe that was uncovered after removal of the tumbleweeds from the northern footprint suction pit. Sample locations for CAS 03-09-02 are shown in Figure A.4-4.

\section{A.4.1.5 Deviations}

Investigation samples were collected as outlined in the CAU 234 CAIP (NNSA/NSO, 2007) and submitted for laboratory analysis with no deviations from the planned sample locations. 


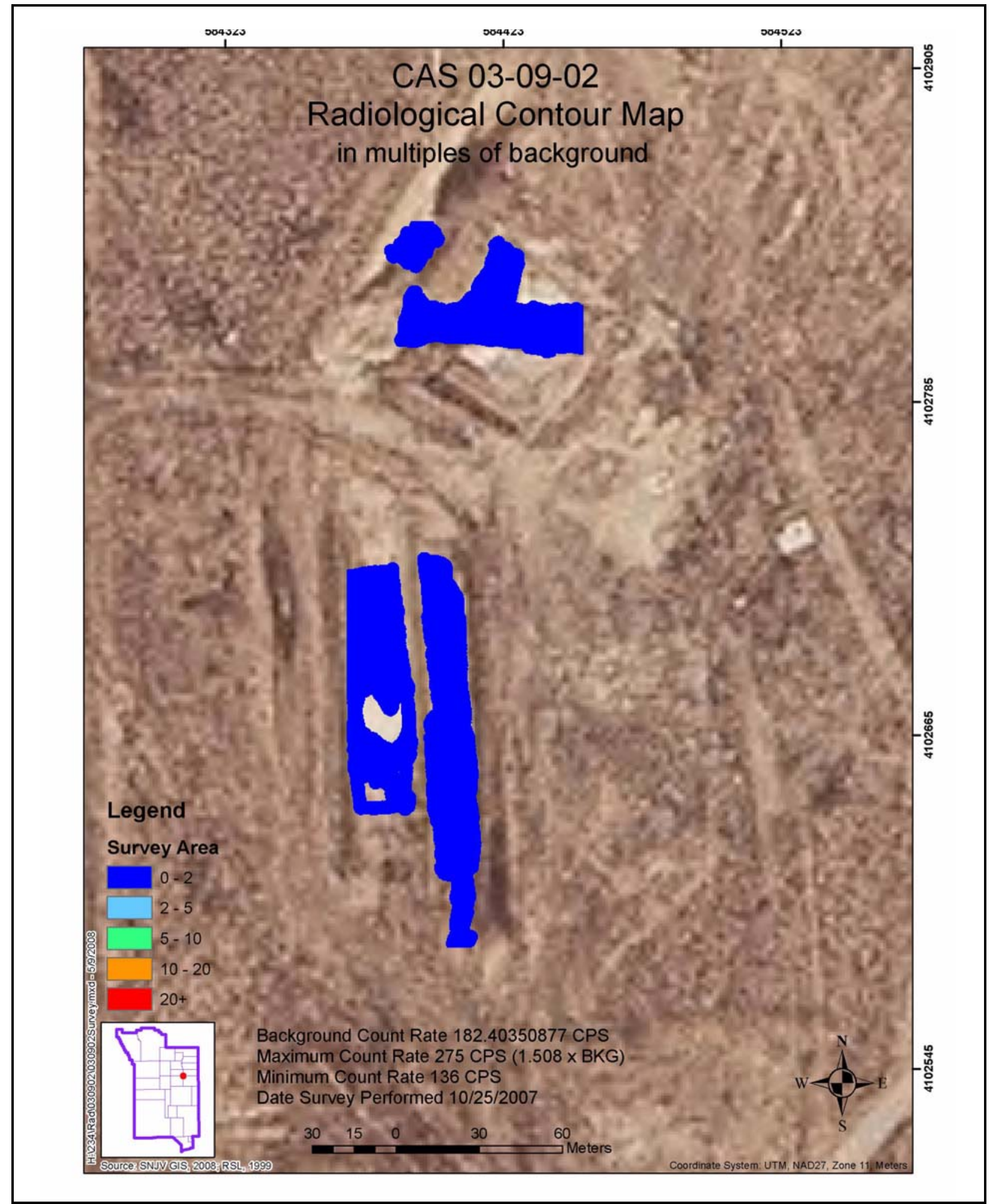

Figure A.4-3

Radiological Survey for CAS 03-09-02 


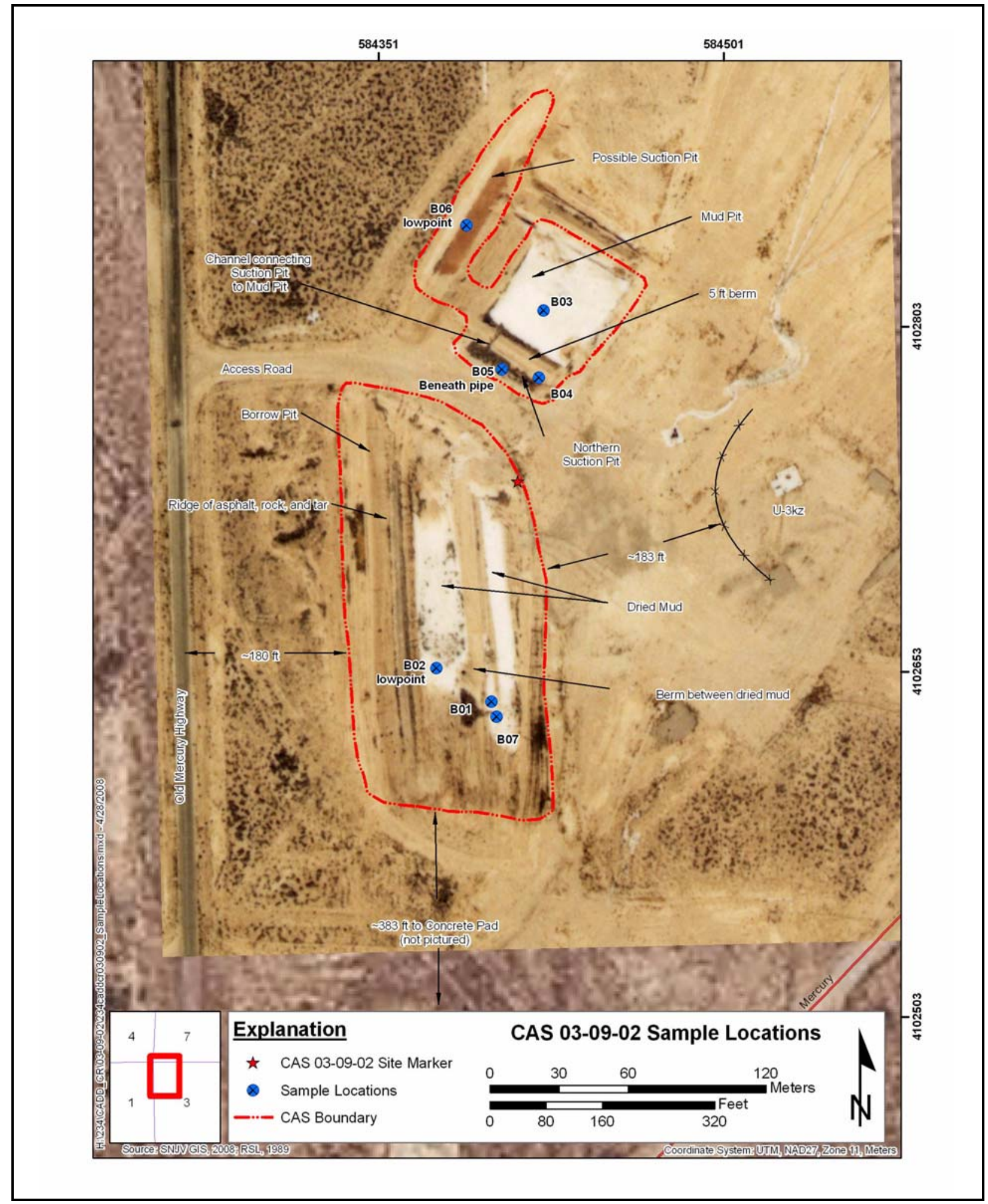

Figure A.4-4

Sample Locations for CAS 03-09-02 


\section{A.4.2 Investigation Results}

The following sections provide analytical results from the samples collected to complete investigation activities as outlined in the CAIP (NNSA/NSO, 2007). Investigation samples were analyzed for the CAIP-specified COPCs, which included VOCs, SVOCs, TPH-DRO, RCRA metals, gamma-emitting radionuclides, isotopic $\mathrm{U}$, isotopic $\mathrm{Pu}$, and $\mathrm{Sr}-90$. The PCBs are added parameters because these contaminants are a common concern at the NTS. The analytical parameters and laboratory methods used to analyze the investigation samples are listed in Table A.2-2. Table A.4-1 lists the sample-specific analytical suite for CAS 03-09-02.

Analytical results from the soil samples with concentrations exceeding MDCs are summarized in the following sections. An evaluation was conducted on all contaminants detected above MDCs by comparing individual concentration or activity results against the FALs. Establishment of the FALs is presented in Appendix C.

\section{A.4.2.1 Volatile Organic Compounds}

The VOCs analytical results for environmental samples collected at this CAS that were detected above MDCs are presented in Table A.4-2. No VOCs were detected at concentrations exceeding their respective PALs. Therefore, the FALs were established at the PAL concentrations.

Table A.4-2

Soil Sample Results for VOCs Detected above Minimum Detectable Concentrations at CAS 03-09-02, Mud Dump Trenches

\begin{tabular}{|c|c|c|c|}
\hline \multirow{2}{*}{$\begin{array}{l}\text { Sample } \\
\text { Location }\end{array}$} & \multirow{2}{*}{$\begin{array}{l}\text { Sample } \\
\text { Number }\end{array}$} & \multirow{2}{*}{$\begin{array}{c}\text { Depth } \\
\text { (ft bgs) }\end{array}$} & Contaminants of Potential Concern $(\mathrm{mg} / \mathrm{kg})$ \\
\hline & & & Acetone \\
\hline \multicolumn{3}{|c|}{ Final Action Levels ${ }^{a}$} & 54,000 \\
\hline B01 & 234B005 & $1.5-2$ & 0.11 \\
\hline
\end{tabular}

aBased on U.S. Environmental Protection Agency, Region 9 Preliminary Remediation Goals (PRGs) (EPA, 2004).

bgs $=$ Below ground surface

$\mathrm{ft}=$ Foot

$\mathrm{mg} / \mathrm{kg}=$ Milligrams per kilogram 


\section{A.4.2.2 Semivolatile Organic Compounds}

The SVOCs analytical results for environmental samples collected at this CAS that were detected above MDCs are presented in Table A.4-3. The constituent present above MDCs was bis(2-ethylhexyl)phthalate, which did not exceed the PAL of 120 milligrams per kilogram (mg/kg). Therefore, the FALs were established at the PAL concentrations.

Table A.4-3

Soil Sample Results for SVOCs Detected above Minimum Detectable Concentrations at CAS 03-09-02, Mud Dump Trenches

\begin{tabular}{||c|c|c|c||}
\hline \multirow{2}{*}{$\begin{array}{c}\text { Sample } \\
\text { Location }\end{array}$} & \multirow{2}{*}{$\begin{array}{c}\text { Sample } \\
\text { Number }\end{array}$} & $\begin{array}{c}\text { Depth } \\
\text { (ft bgs) }\end{array}$ & Contaminants of Potential Concern (mg/kg) \\
\cline { 3 - 4 } & & Bis(2-ethylhexyl)Phthalate \\
\hline \multicolumn{3}{|c|}{ Final Action Levels } & $\mathbf{1 2 0}$ \\
\hline \hline B01 & $234 \mathrm{~B} 002$ & $0-0.5$ & $0.088(\mathrm{~J})$ \\
\hline B02 & $234 \mathrm{~B} 004$ & $1.5-2$ & $0.35(\mathrm{~J})$ \\
\hline
\end{tabular}

aBased on U.S. Environmental Protection Agency, Region 9 Preliminary Remediation Goals (PRGs) (EPA, 2004).

bgs $=$ Below ground surface

$\mathrm{ft}=$ Foot

$\mathrm{mg} / \mathrm{kg}=$ Milligrams per kilogram

$\mathrm{J}=$ Estimated value

\section{A.4.2.3 Total Petroleum Hydrocarbons}

The TPH-DRO analytical results for soil samples collected at this CAS that were detected above MDCs are presented in Table A.4-4. None of the samples exceeded the PAL of $100 \mathrm{mg} / \mathrm{kg}$ for TPH-DRO. Therefore, the FAL was established at the PAL concentration. 
Table A.4-4

Soil Sample Results for TPH-DRO Detected above Minimum Detectable Concentrations at CAS 03-09-02, Mud Dump Trenches

\begin{tabular}{||c|c|c|c||}
\hline \multirow{2}{*}{$\begin{array}{c}\text { Sample } \\
\text { Location }\end{array}$} & \multirow{2}{*}{$\begin{array}{c}\text { Sample } \\
\text { Number }\end{array}$} & $\begin{array}{c}\text { Depth } \\
\text { (ft bgs) }\end{array}$ & Contaminants of Potential Concern (mg/kg) \\
\cline { 3 - 4 } & & & Diesel-Range Organics \\
\hline \multicolumn{2}{||c|}{ Preliminary Action Levels } & $\mathbf{1 0 0}$ \\
\hline \hline B03 & $234 \mathrm{~B} 006$ & $0.0-0.5$ & $4.9(\mathrm{~J})$ \\
\hline B06 & $234 \mathrm{~B} 012$ & $0.0-0.5$ & 53 \\
\hline
\end{tabular}

aBased on Nevada Administrative Code, "Contamination of Soil: Establishment of Action Levels" (NAC, 2006).

bgs $=$ Below ground surface $\mathrm{ft}=$ Foot

$\mathrm{mg} / \mathrm{kg}=$ Milligrams per kilogram

$\mathrm{J}=$ Estimated value

\section{A.4.2.4 RCRA Metals}

The RCRA metals analytical results for environmental samples collected at this CAS that were detected above MDCs are presented in Table A.4-5. No metals were detected at concentrations exceeding their PALs. Therefore, the FALs were established at the corresponding PAL concentrations.

\section{A.4.2.5 Polychlorinated Biphenyls}

Analytical results for the soil samples collected at this CAS indicate that there are no PCBs present at concentrations above their respective MDCs. Therefore, the FALs were established at the corresponding PAL concentrations.

\section{A.4.2.6 Gamma-Emitting Radionuclides}

Gamma-emitting radionuclides analytical results for environmental samples collected at this CAS that were detected above MDCs are presented in Table A.4-6. None of the gamma-emitting radionuclides exceeded their respective PALs. Therefore, the FALs were established at the corresponding PAL concentrations. 
Table A.4-5

Soil Sample Results for RCRA Metals Detected above Minimum Detectable Concentrations at CAS 03-09-02, Mud Dump Trenches

\begin{tabular}{|c|c|c|c|c|c|c|c|c|c|}
\hline \multirow{2}{*}{$\begin{array}{l}\text { Sample } \\
\text { Location }\end{array}$} & \multirow{2}{*}{$\begin{array}{l}\text { Sample } \\
\text { Number }\end{array}$} & \multirow{2}{*}{$\begin{array}{c}\text { Depth } \\
\text { (ft bgs) }\end{array}$} & \multicolumn{7}{|c|}{ Contaminants of Potential Concern $(\mathrm{mg} / \mathrm{kg})$} \\
\hline & & & Arsenic & Barium & Cadmium & Chromium & Lead & Mercury & Selenium \\
\hline \multicolumn{3}{|c|}{ Final Action Levels } & $23^{a}$ & $67,000^{b}$ & $450^{b}$ & $450^{b}$ & $800^{b}$ & $310^{b}$ & $5,100^{b}$ \\
\hline \multirow{3}{*}{ B01 } & 234B001 & $0.0-0.5$ & 3.8 & 210 & -- & 2.9 & 15 & 0.011 & -- \\
\hline & 234B002 & $0.0-0.5$ & 4.4 & 210 & -- & 3.1 & 15 & 0.011 & -- \\
\hline & 234B005 & $1.5-2.0$ & 4.4 & 110 & 0.13 & 7.3 & 8.9 & 0.015 & -- \\
\hline \multirow{2}{*}{ B02 } & 234B003 & $0.0-0.5$ & 4.6 & 230 & -- & 3.4 & 15 & 0.018 & -- \\
\hline & 234B004 & $1.5-2.0$ & 4.4 & 120 & 0.15 & 6.1 & 9.9 & 0.034 & 0.55 \\
\hline \multirow{2}{*}{ B03 } & 234B006 & $0.0-0.5$ & 3.3 & 170 & 0.22 & 7 & 17 & 0.016 & -- \\
\hline & 234B007 & $1.5-2.0$ & 4.4 & 110 & 0.13 & 5.6 & 11 & 0.023 & -- \\
\hline \multirow{2}{*}{ B04 } & 234B008 & $0.0-0.5$ & 4.5 & 190 & 0.18 & 6.9 & 16 & 0.0086 & -- \\
\hline & 234B009 & $1.5-2.0$ & 2.3 & 82 & 0.093 & 1.6 & 6.7 & 0.0072 & -- \\
\hline \multirow{2}{*}{ B05 } & 234B010 & $0.0-0.5$ & 2.9 & 230 & 0.19 & 4.4 & 14 & 0.0096 & -- \\
\hline & 234B011 & $1.5-2.0$ & 3.7 & 100 & 0.13 & 5.6 & 7.7 & 0.0062 & -- \\
\hline \multirow{2}{*}{ B06 } & 234B012 & $0.0-0.5$ & 9.5 & 310 & -- & 8.7 & 16 & 0.022 & -- \\
\hline & 234B013 & $1.5-2.0$ & 4 & 120 & -- & 4.1 & 7.3 & 0.019 & -- \\
\hline B07 & 234B014 & $0.0-0.5$ & 4.8 & 220 & -- & 4 & 17 & -- & 0.53 \\
\hline
\end{tabular}

aBased on the background concentrations for metals. Background is considered the mean plus two times the standard deviation for sediment samples collected by the Nevada Bureau of Mines and Geology throughout the Nevada Test and Training Range (NBMG, 1998; Moore, 1999).

bBased on U.S. Environmental Protection Agency, Region 9 Preliminary Remediation Goals (PRGs) (EPA, 2004).

bgs $=$ Below ground surface

$\mathrm{ft}=$ Foot

$\mathrm{mg} / \mathrm{kg}=$ Milligrams per kilogram

$--=$ Not detected above minimum detectable concentrations 
Table A.4-6

Soil Sample Results for Gamma-Emitting Radionuclides Detected above Minimum Detectable Concentrations at CAS 03-09-02, Mud Dump Trenches (Page 1 of 2)

\begin{tabular}{|c|c|c|c|c|c|c|c|c|c|c|c|c|}
\hline \multirow{2}{*}{$\begin{array}{l}\text { Sample } \\
\text { Location }\end{array}$} & \multirow{2}{*}{$\begin{array}{l}\text { Sample } \\
\text { Number }\end{array}$} & \multirow{3}{*}{$\begin{array}{c}\text { Depth } \\
\text { (ft bgs) }\end{array}$} & \multicolumn{10}{|c|}{ Contaminants of Potential Concern $(\mathrm{pCi} / \mathrm{g})$} \\
\hline & & & \multicolumn{2}{|c|}{ Actinium-228 } & \multirow{2}{*}{$\frac{\text { Cesium-137 }}{12.2^{\mathrm{b}}}$} & \multicolumn{2}{|c|}{ Lead-212 } & \multicolumn{2}{|c|}{ Lead-214 } & \multicolumn{2}{|c|}{ Thallium-208 } & \multirow{2}{*}{$\frac{\text { Thorium-234 }}{105^{\mathrm{b}}}$} \\
\hline \multicolumn{2}{|c|}{ Final Action Levels } & & $5^{a}$ & $15^{a}$ & & $5^{a}$ & $15^{a}$ & $5^{a}$ & $15^{a}$ & $5^{a}$ & $15^{a}$ & \\
\hline \multirow{3}{*}{ B01 } & 234B001 & $0.0-0.5$ & 3.01 & -- & -- & $2.7(\mathrm{~J})$ & -- & $1.24(\mathrm{~J})$ & -- & 0.95 & -- & -- \\
\hline & 234B002 & $0.0-0.5$ & 2.31 & -- & -- & $3.07(\mathrm{~J})$ & -- & $1.39(\mathrm{~J})$ & -- & 0.96 & -- & -- \\
\hline & 234B005 & $1.5-2.0$ & -- & 1.62 & -- & -- & $1.73(\mathrm{~J})$ & -- & $1.07(\mathrm{~J})$ & -- & 0.56 & -- \\
\hline \multirow{2}{*}{ B02 } & 234B003 & $0.0-0.5$ & 2.48 & -- & -- & $3.25(J)$ & -- & $1.33(\mathrm{~J})$ & -- & 0.79 & -- & -- \\
\hline & 234B004 & $1.5-2.0$ & -- & 1.54 & -- & -- & $1.93(\mathrm{~J})$ & -- & $1.25(J)$ & -- & 0.68 & -- \\
\hline \multirow{2}{*}{ B03 } & 234B006 & $0.0-0.5$ & 2.59 & -- & -- & $2.43(\mathrm{~J})$ & -- & $1.23(\mathrm{~J})$ & -- & 0.65 & -- & -- \\
\hline & 234B007 & $1.5-2.0$ & -- & 1.62 & -- & -- & $2.24(\mathrm{~J})$ & -- & $1.29(\mathrm{~J})$ & -- & 0.65 & -- \\
\hline \multirow{2}{*}{ B04 } & 234B008 & $0.0-0.5$ & 2.89 & -- & 2.7 & 3.27 (J) & -- & $1.23(\mathrm{~J})$ & -- & 0.96 & -- & -- \\
\hline & 234B009 & $1.5-2.0$ & -- & 2.87 & -- & -- & $3.05(\mathrm{~J})$ & -- & 1.57 (J) & -- & 0.98 & -- \\
\hline \multirow{2}{*}{ B05 } & 234B010 & $0.0-0.5$ & 1.28 & -- & 1.1 & $1.88(\mathrm{~J})$ & -- & $1.21(\mathrm{~J})$ & -- & 0.66 & -- & -- \\
\hline & 234B011 & $1.5-2.0$ & -- & 1.36 & -- & -- & $1.59(\mathrm{~J})$ & -- & $1.31(\mathrm{~J})$ & -- & 0.7 & -- \\
\hline \multirow{2}{*}{ B06 } & 234B012 & $0.0-0.5$ & 2.31 & -- & -- & $2.64(J)$ & & $1.2(\mathrm{~J})$ & -- & 0.74 & -- & -- \\
\hline & 234B013 & $1.5-2.0$ & -- & 1.53 & -- & -- & $1.81(\mathrm{~J})$ & -- & $1.2(\mathrm{~J})$ & -- & 0.54 & -- \\
\hline B07 & 234B014 & $0.0-0.5$ & 3.49 & -- & -- & 3.75 & -- & 1.74 & -- & 1.14 & -- & 4.72 \\
\hline
\end{tabular}


Table A.4-6

Soil Sample Results for Gamma-Emitting Radionuclides Detected above Minimum Detectable Concentrations at CAS 03-09-02, Mud Dump Trenches (Page 2 of 2)

\begin{tabular}{|c|c|c|c|c|c|c|c|c|c|c|c|c|}
\hline \multirow{2}{*}{$\begin{array}{l}\text { Sample } \\
\text { Location }\end{array}$} & \multirow{2}{*}{$\begin{array}{l}\text { Sample } \\
\text { Number }\end{array}$} & \multirow{2}{*}{$\begin{array}{c}\text { Depth } \\
\text { (ft bgs) }\end{array}$} & \multicolumn{10}{|c|}{ Contaminants of Potential Concern $(\mathrm{pCi} / \mathrm{g})$} \\
\hline & & & \multicolumn{2}{|c|}{ Actinium-228 } & \multirow{2}{*}{$\frac{\text { Cesium-137 }}{12.2^{\mathrm{b}}}$} & \multicolumn{2}{|c|}{ Lead-212 } & \multicolumn{2}{|c|}{ Lead-214 } & \multicolumn{2}{|c|}{ Thallium-208 } & \multirow{2}{*}{$\begin{array}{c}\text { Thorium-234 } \\
105^{\mathrm{b}}\end{array}$} \\
\hline & Action L & & $5^{a}$ & $15^{\mathrm{a}}$ & & $5^{a}$ & $15^{\mathrm{a}}$ & $5^{a}$ & $15^{\mathrm{a}}$ & $5^{a}$ & $15^{\mathrm{a}}$ & \\
\hline
\end{tabular}

${ }^{a}$ Taken from the generic guidelines for residual concentrations of actinium-228, bismuth-214, lead-212, lead-214, thallium-208, and thorium-232, as found in Chapter IV of DOE Order 5400.5, Change 2, "Radiation Protection of the Public and Environment." (DOE, 1993). The PALs for these isotopes are specified as $5 \mathrm{pCi} / \mathrm{g}$ averaged over the first $15 \mathrm{~cm}$ of soil and $15 \mathrm{pCi} / \mathrm{g}$ for deeper soils (DOE, 1993). For purposes of this document, $15 \mathrm{~cm}$ is assumed to be equivalent to $0.5 \mathrm{ft}$ ( 6 inches); therefore, $5 \mathrm{pCi} / \mathrm{g}$ represents the PALs for these radionuclides in the surface soil ( 0 to $0.5 \mathrm{ft}$ depth).

${ }^{6}$ Taken from the construction, commercial, industrial land use scenario in Table 2.1 of the NCRP Report No. 129, Recommended Screening Limits for Contaminated Surface Soil and Review Factors Relevant to Site-Specific Studies (NCRP, 1999). The values provided in this source document were scaled to a 25 -millirem-per-year dose.

bgs $=$ Below ground surface

$\mathrm{cm}=$ Centimeter

DOE $=$ U.S. Department of Energy

$\mathrm{ft}=$ Foot

NCRP $=$ National Council on Radiation Protection and Measurements

$\mathrm{PAL}=$ Preliminary action level

$\mathrm{pCi} / \mathrm{g}=$ Picocuries per gram

$\mathrm{J}=$ Estimated value

-- = Not detected above minimum detectable concentrations 


\section{A.4.2.7 Plutonium, Strontium-90, and Uranium Isotopes}

Isotopic $\mathrm{Pu}$ and isotopic $\mathrm{U}$ analytical results for environmental samples collected at this CAS that were detected above MDCs are presented in Table A.4-7. No Sr-90 was identified above its MDC in any of the samples analyzed. None of the $\mathrm{Pu}, \mathrm{U}$, or $\mathrm{Sr}-90$ isotope results exceeded their respective PALs. Therefore, the FALs were established at the corresponding PAL concentrations.

Table A.4-7

Soil Sample Results for Isotopes Detected above Minimum Detectable Concentrations at CAS 03-09-02, Mud Dump Trenches

\begin{tabular}{|c|c|c|c|c|c|c|}
\hline \multirow{2}{*}{$\begin{array}{l}\text { Sample } \\
\text { Location }\end{array}$} & \multirow{2}{*}{$\begin{array}{l}\text { Sample } \\
\text { Number }\end{array}$} & \multirow{2}{*}{$\begin{array}{c}\text { Depth } \\
\text { (ft bgs) }\end{array}$} & \multicolumn{4}{|c|}{ Contaminants of Potential Concern $(\mathrm{pCi} / \mathrm{g})$} \\
\hline & & & Plutonium-239/240 & Uranium-234 & Uranium-235 & Uranium-238 \\
\hline \multicolumn{3}{|c|}{ Final Action Levels ${ }^{a}$} & 12.7 & 143 & 17.6 & 105 \\
\hline \multirow{3}{*}{ B01 } & 234B001 & $0.0-0.5$ & 0.239 & 1.21 & -- & 1.46 \\
\hline & 234B002 & $0.0-0.5$ & 0.08 & 1.17 & 0.062 & 1.46 \\
\hline & 234B005 & $1.5-2.0$ & -- & 1.23 & 0.048 & 1.3 \\
\hline \multirow{2}{*}{ B02 } & 234B003 & $0.0-0.5$ & 0.112 & 1.33 & 0.094 & 1.42 \\
\hline & 234B004 & $1.5-2.0$ & 0.071 & 0.96 & 0.061 & 1.13 \\
\hline \multirow{2}{*}{ B03 } & 234B006 & $0.0-0.5$ & 0.046 & 1.33 & 0.061 & 1.19 \\
\hline & 234B007 & $1.5-2.0$ & -- & 1.2 & 0.057 & 1.26 \\
\hline \multirow{2}{*}{ B04 } & 234B008 & $0.0-0.5$ & -- & 1.44 & 0.063 & 1.56 \\
\hline & 234B009 & $1.5-2.0$ & -- & 1.19 & 0.078 & 1.33 \\
\hline \multirow{2}{*}{ B05 } & 234B010 & $0.0-0.5$ & 0.038 & 0.99 & 0.062 & 1.11 \\
\hline & 234B011 & $1.5-2.0$ & -- & 1.16 & 0.071 & 1.39 \\
\hline \multirow{2}{*}{ B06 } & 234B012 & $0.0-0.5$ & 0.169 & 1.28 & 0.047 & 1.38 \\
\hline & 234B013 & $1.5-2.0$ & -- & 1.02 & -- & 1.01 \\
\hline B07 & 234B014 & $0.0-0.5$ & $0.095(\mathrm{~J})$ & 1.31 & -- & 1.52 \\
\hline
\end{tabular}

${ }^{a}$ Taken from the construction, commercial, industrial land use scenario in Table 2.1 of the NCRP Report No. 129, Recommended Screening Limits for Contaminated Surface Soil and Review Factors Relevant to Site-Specific Studies (NCRP, 1999). The values provided in this source document were scaled to a 25-millirem-per-year dose.

bgs $=$ Below ground surface

$\mathrm{ft}=$ Foot

NCRP $=$ National Council on Radiation Protection and Measurements

$\mathrm{pCi} / \mathrm{g}=$ Picocuries per gram

$\mathrm{J}=$ Estimated

$--=$ Not detected above minimum detectable concentrations 


\section{A.4.3 Nature and Extent of Contamination}

Based on the analytical results for soil samples collected within CAS 03-09-02, none of the samples exceeded the FALs for any of the analyses. Therefore, no COCs are present at this CAS.

\section{A.4.4 Revised Conceptual Site Model}

The results of the CAI at CAS 03-09-02 were consistent with the CSM. No revision of the CSM was necessary. 


\section{A.5.0 Corrective Action Site 12-09-01, Mud Pit}

Corrective Action Site 12-09-01 consists of three distinct footprints in Area 12 of the NTS. The three footprints contain a mud pit, a piece of metal pipe, and a large cylindrical piece of metal debris (Figure A.5-1). The mud pit is associated with the drilling of the U12r PS\#1A post-test cellar that was completed in December 1968. The mud pit is approximately $30 \mathrm{ft}$ to the west of the cellar and is approximately 100 by $25 \mathrm{ft}$ in area. The mud pit contains dry, cracked mud and little vegetation.

The piece of metal pipe and the cylinder are located approximately $60 \mathrm{ft}$ to the northwest of the mud pit. The entire length of metal pipe is lying on the surface. The metal cylinder is lying on its side and contains an open bottom that has been covered by a metal grating, and an hole on the side of the cylinder that once had a hinged door that covered the opening. Within the metal cylinder are rusted cans and broken bottles, along with some small pieces of paper debris. It is unknown when or why the piece of pipe and metal cylinder were placed at the site. The soil beneath the debris was the scope of the CAI and investigated for impact due to potential for releases from the debris.

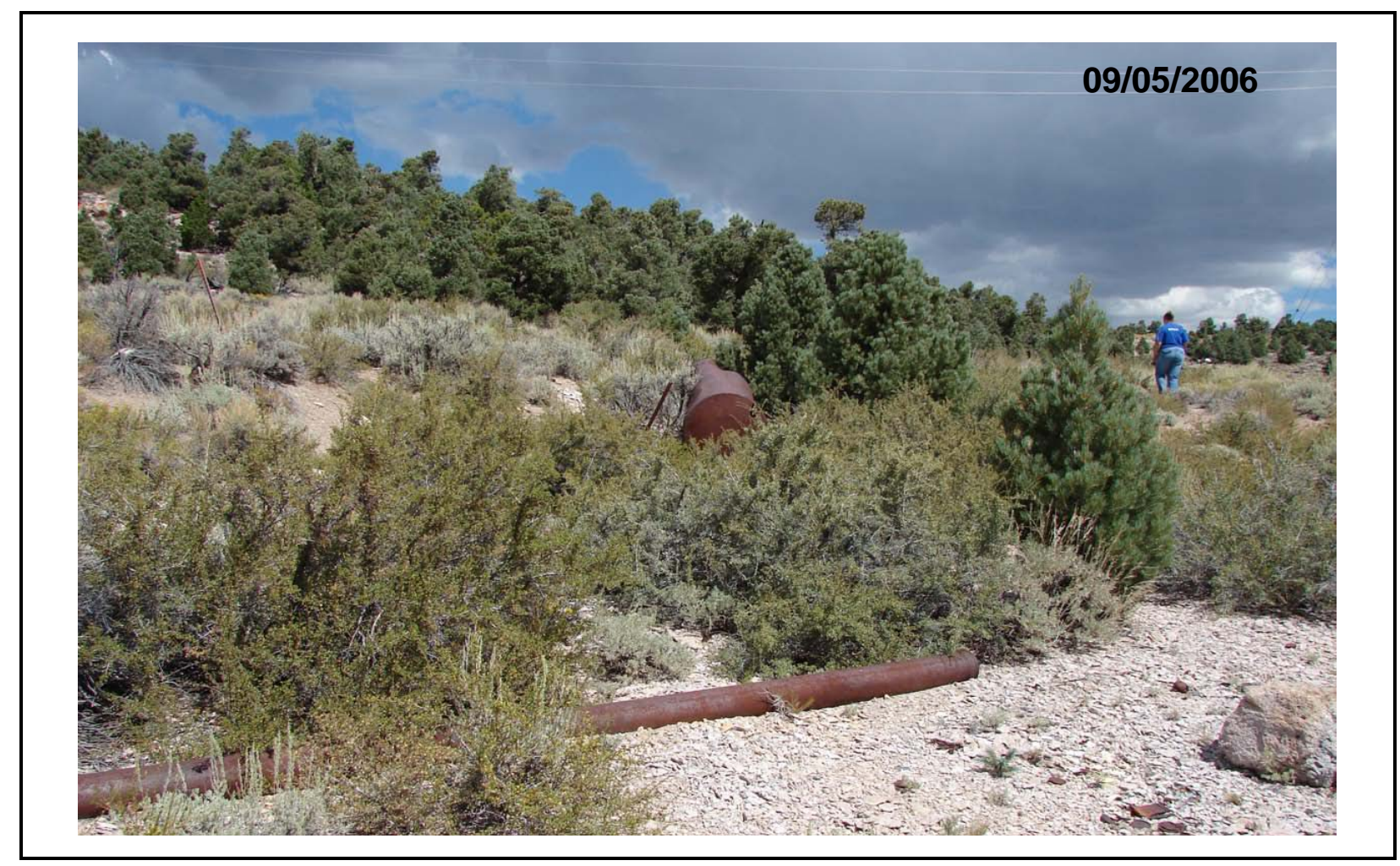

Figure A.5-1

Debris at CAS 12-09-01 


\section{A.5.1 Corrective Action Investigation}

A total of six characterization samples (including one FD) were collected during investigation activities at CAS 12-09-01. The sample IDs, locations, types, and analyses are listed in Table A.5-1. The specific CAI activities conducted to satisfy the CAIP requirements at this CAS (NNSA/NSO, 2007) are described in the following sections.

Table A.5-1

Samples Collected at CAS 12-09-01, Mud Pit

\begin{tabular}{||c|c|c|c|c|c|}
\hline $\begin{array}{c}\text { Sample } \\
\text { Location }\end{array}$ & $\begin{array}{c}\text { Sample } \\
\text { Number }\end{array}$ & $\begin{array}{c}\text { Depth } \\
\text { (ft bgs) }\end{array}$ & Matrix & Purpose & Analyses \\
\hline \hline \multirow{2}{*}{ C01 } & $234 \mathrm{C} 001$ & $0.0-0.33$ & Soil & Environmental & Set 1 \\
\cline { 2 - 6 } & $234 \mathrm{C} 002$ & $0.0-0.33$ & Soil & Field Duplicate of 234C001 & Set 1 \\
\hline C02 & $234 \mathrm{C} 003$ & $0.0-0.33$ & Soil & Environmental & Set 1 \\
\hline C03 & $234 \mathrm{C} 004$ & $0.0-0.5$ & Soil & Environmental & Set 1 \\
\hline C04 & $234 \mathrm{C} 005$ & $0.0-0.5$ & Soil & Environmental & Set 1 \\
\hline C05 & $234 \mathrm{C} 006$ & $0.0-0.5$ & Soil & Environmental & Set 1 \\
\hline N/A & $234 C 301$ & N/A & Water & Trip Blank & VOCs only \\
\hline
\end{tabular}

Set 1 = VOCs, SVOCs, RCRA Metals, TPH-DRO, PCBs, Gamma Spectroscopy, Isotopic Uranium, Isotopic Plutonium, Strontium-90

bgs $=$ Below ground surface

$\mathrm{DRO}=$ Diesel-range organics

$\mathrm{ft}=$ Foot

$\mathrm{N} / \mathrm{A}=$ Not applicable

$\mathrm{PCB}=$ Polychlorinated biphenyl
RCRA $=$ Resource Conservation and Recovery Act

SVOC = Semivolatile organic compound

$\mathrm{TPH}=$ Total petroleum hydrocarbons

$\mathrm{VOC}=$ Volatile organic compound

\section{A.5.1.1 Field Screening}

Decision I soil samples from each CAS were field screened for alpha and beta/gamma radiation as specified in the CAU 234 CAIP (NNSA/NSO, 2007). The FSRs were compared to FSLs to guide subsequent sampling decisions where appropriate. Alpha and beta/gamma radiation FSLs were not exceeded during sampling activities. Therefore, no additional samples were collected. 


\section{A.5.1.2 Radiological Surveys}

An aerial radiological survey was conducted in 1994 of Area 12, including CAS 12-09-01 (BN, 1999). The findings of the survey were indistinguishable from background. Therefore, no additional samples were collected (see Figure A.5-2).

\section{A.5.1.3 Visual Inspections}

Two features associated with the CAS were identified. The first feature is a length of metal piping (approximately $15 \mathrm{ft}$ in length) resting on the ground with no connections at either end. The second feature is a large cylindrical metal debris that is lying on its side. The cylinder has a diameter of approximately 40 inches and is approximately $8 \mathrm{ft}$ long. The bottom of the cylinder is cut out, and a square opening on the side of the cylinder indicates the presence of an opening that once had a hinged cover for access. Currently, burned debris is located within the cylinder at the square opening, including glass and metal.

\section{A.5.1.4 Sample Collection}

Decision I sampling activities at CAS 12-09-01 included the collection of environmental soil samples from five locations identified in Figure A.5-3.

Soil samples were collected using scoops for surface samples and hand augers for subsurface samples. Refusal (volcanic tuff) was encountered at a depth of $0.33 \mathrm{ft}$ bgs for all locations around the metal pipe and at $0.5 \mathrm{ft}$ bgs at all locations around the cylindrical metal debris.

\section{A.5.1.5 Deviations}

The deviations to the sampling plans for CAS 12-09-01 investigation identified in the CAU 234 CAIP (NNSA/NSO, 2007) pertained to the depth to which samples could be collected. As indicated in Section A.5.1.4, refusal was encountered at a relatively shallow depth. Instead of the planned depth of $1 \mathrm{ft}$ bgs, the maximum depth for samples collected around the metal pipe and the cylindrical metal debris were only $0.33 \mathrm{ft}$ bgs and $0.5 \mathrm{ft}$ bgs, respectively. This did not impact DQO decisions as no COCs are present within these surface samples; therefore, no additional samples were required. 


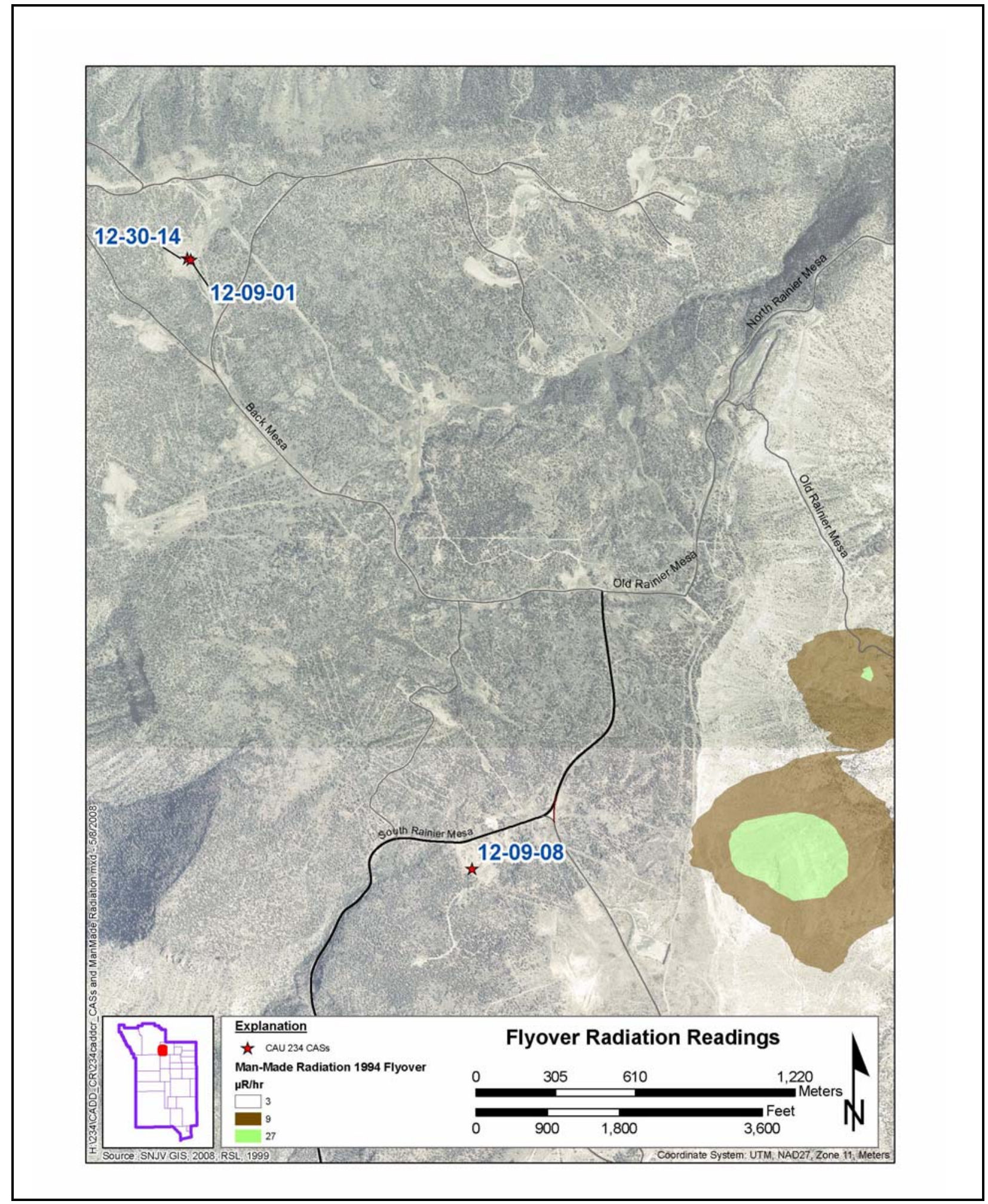

Figure A.5-2

Flyover Radiation Readings 


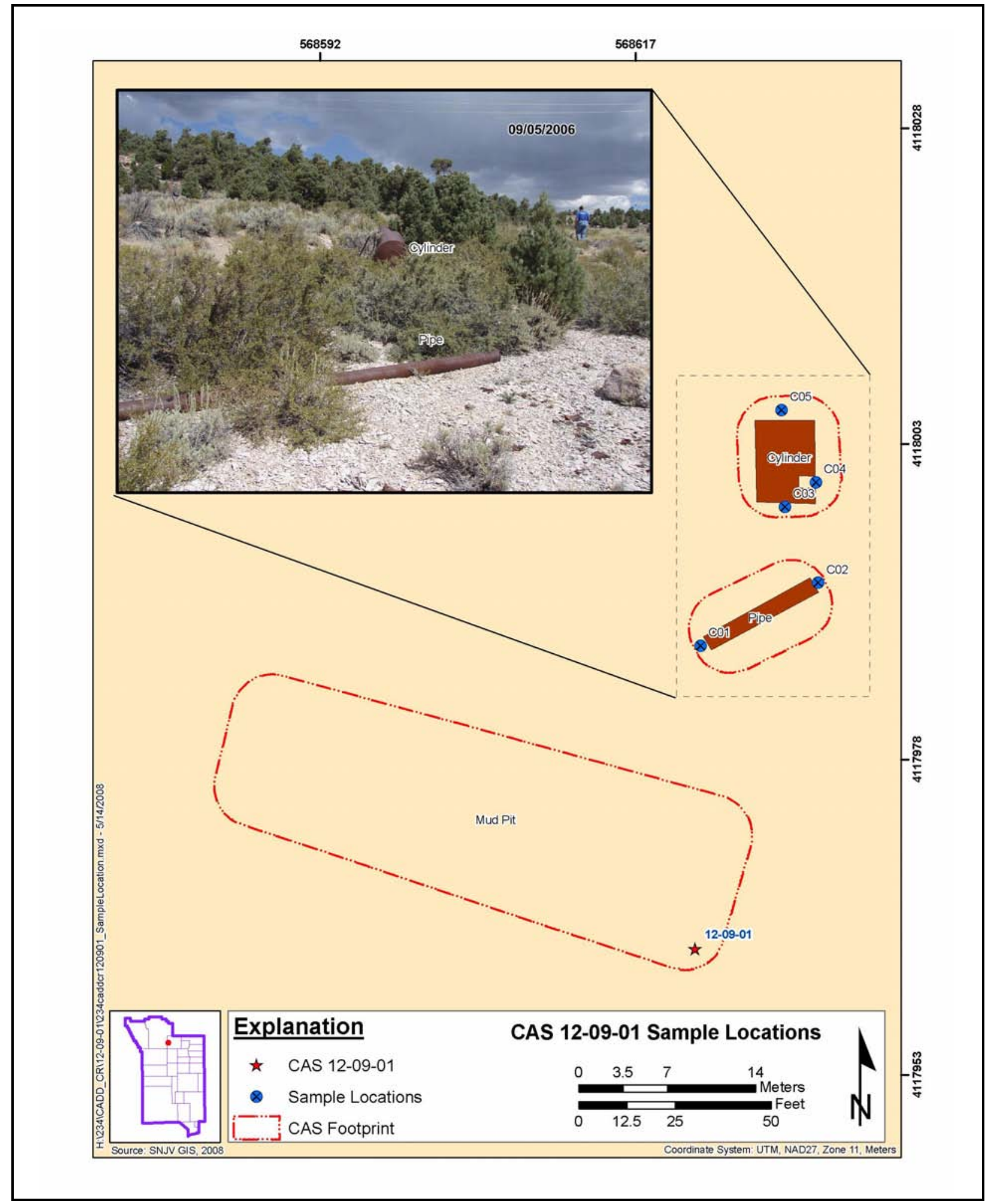

Figure A.5-3

Sample Locations for CAS 12-09-01 


\section{A.5.2 Investigation Results}

The following sections provide analytical results from the samples collected to complete investigation activities as outlined in the CAIP (NNSA/NSO, 2007). Investigation samples were analyzed for the CAIP-specified COPCs, which included VOCs, SVOCs, TPH-DRO, RCRA metals, gamma-emitting radionuclides, isotopic $\mathrm{U}$, isotopic $\mathrm{Pu}$, and $\mathrm{Sr}-90$. The PCBs are added parameters because these contaminants are a common concern at the NTS. The analytical parameters and laboratory methods used to analyze the investigation samples are listed in Table A.2-2. Table A.5-1 lists the sample-specific analytical suite for CAS 12-09-01.

Analytical results from the soil samples with concentrations exceeding MDCs are summarized in the following sections. An evaluation was conducted on all contaminants detected above MDCs by comparing individual concentration or activity results against the FALs. Establishment of the FALs is presented in Appendix D.

\section{A.5.2.1 Volatile Organic Compounds}

The VOCs analytical results for environmental samples collected at this CAS that were detected above MDCs are presented in Table A.5-2. None of the sample results were above their respective PALs. Therefore, the FALs were established at their corresponding PAL concentrations.

Table A.5-2

Soil Sample Results for VOCs Detected above Minimum Detectable Concentrations at CAS 12-09-01, Mud Pit

\begin{tabular}{|c|c|c|c|}
\hline \multirow{2}{*}{$\begin{array}{c}\text { Sample } \\
\text { Location }\end{array}$} & $\begin{array}{c}\text { Sample } \\
\text { Number }\end{array}$ & $\begin{array}{c}\text { Depth } \\
\text { (ft bgs) }\end{array}$ & Contaminants of Potential Concern (mg/kg) \\
\cline { 3 - 4 } & & p-lsopropyltoluene \\
\hline \multicolumn{3}{|c|}{ Final Action Levels } & $\mathbf{2 , 0 0 0}$ \\
\hline \hline $\mathrm{C} 03$ & $234 \mathrm{C} 004$ & $0.0-0.5$ & $0.0022(\mathrm{~J})$ \\
\hline
\end{tabular}

aBased on U.S. Environmental Protection Agency, Region 9 Preliminary Remediation Goals (PRGs) (EPA, 2004).

bgs $=$ Below ground surface $\mathrm{ft}=$ Foot

$\mathrm{mg} / \mathrm{kg}=$ Milligrams per kilogram

$\mathrm{J}=$ Estimated value 


\section{A.5.2.2 Semivolatile Organic Compounds}

The SVOCs analytical results for environmental samples collected at this CAS that were detected above MDCs are presented in Table A.5-3. None of the sample results were above their respective PALs. Therefore, the FALs were established at their corresponding PAL concentrations.

Table A.5-3

\section{Soil Sample Results for SVOCs Detected above Minimum Detectable Concentrations at CAS 12-09-01, Mud Pit}

\begin{tabular}{||c|c|c|c|c||}
\hline \multirow{2}{*}{$\begin{array}{c}\text { Sample } \\
\text { Location }\end{array}$} & \multirow{2}{*}{$\begin{array}{c}\text { Sample } \\
\text { Number }\end{array}$} & \multirow{2}{*}{$\begin{array}{c}\text { Depth } \\
\text { (ft bgs) }\end{array}$} & \multicolumn{2}{|c|}{ Contaminants of Potential Concern (mg/kg) } \\
\cline { 4 - 5 } & & & Benzo(b)Fluoranthene & Bis(2-Ethylhexyl)Phthalate \\
\hline \multicolumn{3}{|c|}{ Final Action Levels ${ }^{\mathrm{a}}$} & 2.1 & 120 \\
\hline \hline \multirow{2}{*}{$\mathrm{C} 01$} & $234 \mathrm{C} 001$ & $0.0-0.33$ & $0.074(\mathrm{~J})$ & $0.12(\mathrm{~J})$ \\
\cline { 2 - 5 } & $234 \mathrm{C} 002$ & $0.0-0.33$ & $0.086(\mathrm{~J})$ & $0.075(\mathrm{~J})$ \\
\hline
\end{tabular}

aBased on U.S. Environmental Protection Agency, Region 9 Preliminary Remediation Goals (PRGs) (EPA, 2004).

bgs $=$ Below ground surface

$\mathrm{ft}=$ Foot

$\mathrm{mg} / \mathrm{kg}=$ Milligrams per kilogram

$\mathrm{J}=$ Estimated value

\section{A.5.2.3 Total Petroleum Hydrocarbons}

The TPH-DRO analytical results for soil samples collected at this CAS that were detected above MDCs are presented in Table A.5-4. None of the sample results were above the PAL. Therefore, the FAL was established at the PAL concentration.

\section{A.5.2.4 RCRA Metals}

RCRA metals analytical results for environmental samples collected at this CAS that were detected above MDCs are presented in Table A.5-5. None of the RCRA metals were detected above their respective PALs. Therefore, the FALs were established at their corresponding PAL concentrations.

\section{A.5.2.5 Polychlorinated Biphenyls}

Analytical results for the soil samples collected at this CAS indicate that there are no PCBs detected above their respective MDCs. Therefore, the FALs were established at the PAL concentrations. 
Table A.5-4

Soil Sample Results for TPH-DRO Detected above

Minimum Detectable Concentrations at CAS 12-09-01, Mud Pit

\begin{tabular}{||c|c|c|c||}
\hline \multirow{2}{*}{$\begin{array}{c}\text { Sample } \\
\text { Location }\end{array}$} & \multirow{2}{*}{$\begin{array}{c}\text { Sample } \\
\text { Number }\end{array}$} & \multirow{2}{*}{$\begin{array}{c}\text { Depth } \\
\text { (ft bgs) }\end{array}$} & Contaminants of Potential Concern (mg/kg) \\
\cline { 3 - 4 } & & & Diesel-Range Organics \\
\hline \multicolumn{2}{|c|}{ Preliminary Action Levels } & $\mathbf{1 0 0}$ \\
\hline \hline \multirow{2}{*}{ C01 } & $234 \mathrm{C} 001$ & $0.0-0.33$ & 7 \\
\cline { 2 - 5 } & $234 \mathrm{C} 002$ & $0.0-0.33$ & 5.4 \\
\hline $\mathrm{C} 03$ & $234 \mathrm{C} 004$ & $0.0-0.5$ & $1.9(\mathrm{~J})$ \\
\hline
\end{tabular}

aBased on Nevada Administrative Code, "Contamination of Soil: Establishment of Action Levels" (NAC, 2006).

bgs $=$ Below ground surface

$\mathrm{ft}=$ Foot

$\mathrm{mg} / \mathrm{kg}=$ Milligrams per kilogram

$\mathrm{J}=$ Estimated value

Table A.5-5

Soil Sample Results for RCRA Metals Detected above Minimum Detectable Concentrations at CAS 12-09-01, Mud Pit

\begin{tabular}{|c|c|c|c|c|c|c|c|c|c|c|}
\hline \multirow{2}{*}{$\begin{array}{c}\text { Sample } \\
\text { Location }\end{array}$} & \multirow{2}{*}{$\begin{array}{l}\text { Sample } \\
\text { Number }\end{array}$} & \multirow{2}{*}{$\begin{array}{l}\text { Depth } \\
\text { (ft bgs) }\end{array}$} & \multicolumn{8}{|c|}{ Contaminants of Potential Concern $(\mathrm{mg} / \mathrm{kg})$} \\
\hline & & & Arsenic & Barium & Cadmium & Chromium & Lead & Mercury & Selenium & Silver \\
\hline \multicolumn{3}{|c|}{ Final Action Levels } & $23^{\mathrm{a}}$ & $67,000^{b}$ & $450^{b}$ & $450^{b}$ & $800^{b}$ & $310^{b}$ & $5,100^{b}$ & $5,100^{\mathrm{b}}$ \\
\hline \multirow{2}{*}{$\mathrm{C} 01$} & $234 \mathrm{C} 001$ & $0.0-0.33$ & 2.3 & 120 & -- & 5.3 & 20 & -- & 0.37 & -- \\
\hline & $234 \mathrm{C} 002$ & $0.0-0.33$ & 2.2 & 120 & -- & 5 & 23 & -- & 0.46 & 0.2 \\
\hline $\mathrm{C} 02$ & $234 \mathrm{C} 003$ & $0.0-0.33$ & 2.6 & 42 & -- & 4.5 & 18 & -- & -- & -- \\
\hline $\mathrm{C} 03$ & $234 \mathrm{C} 004$ & $0.0-0.5$ & 2.9 & 65 & 0.13 & 5.4 & $20(\mathrm{~J})$ & 0.02 & -- & -- \\
\hline C04 & $234 \mathrm{C} 005$ & $0.0-0.5$ & 2.5 & 68 & 0.064 & 5 & $14(\mathrm{~J})$ & 0.026 & -- & -- \\
\hline C05 & $234 \mathrm{C} 006$ & $0.0-0.5$ & 3.7 & 75 & 0.14 & 7.4 & $33(\mathrm{~J})$ & 0.018 & -- & -- \\
\hline
\end{tabular}

aBased on the background concentrations for metals. Background is considered the mean plus two times the standard deviation for sediment samples collected by the Nevada Bureau of Mines and Geology throughout the Nevada Test and Training Range (NBMG, 1998; Moore, 1999).

bBased on U.S. Environmental Protection Agency, Region 9 Preliminary Remediation Goals (PRGs) (EPA, 2004).

bgs $=$ Below ground surface

$\mathrm{ft}=$ Foot

$\mathrm{mg} / \mathrm{kg}=$ Milligrams per kilogram

$\mathrm{J}=$ Estimated value

$--=$ Not detected above minimum detectable concentrations 


\section{A.5.2.6 Gamma-Emitting Radionuclides}

Gamma-emitting radionuclides analytical results for environmental samples collected at this CAS that were detected above MDCs are presented in Table A.5-6. None of the gamma-emitting radionuclides were detected above their respective PALs. Therefore, the FALs were established as their corresponding PAL concentrations.

Table A.5-6

Soil Sample Results for Gamma-Emitting Radionuclides Detected above Minimum Detectable Concentrations at CAS 12-09-01, Mud Pit

\begin{tabular}{|c|c|c|c|c|c|c|c|}
\hline \multirow{2}{*}{$\begin{array}{c}\text { Sample } \\
\text { Location }\end{array}$} & \multirow{2}{*}{$\begin{array}{l}\text { Sample } \\
\text { Number }\end{array}$} & \multirow{2}{*}{$\begin{array}{c}\text { Depth } \\
\text { (ft bgs) }\end{array}$} & \multicolumn{5}{|c|}{ Contaminants of Potential Concern (pCi/g) } \\
\hline & & & Actinium-228 & Cesium-137 & Lead-212 & Lead-214 & Thallium-208 \\
\hline \multicolumn{3}{|c|}{ Final Action Levels } & $5^{a}$ & $12.2^{b}$ & $5^{\mathrm{a}}$ & $5^{a}$ & $5^{\mathrm{a}}$ \\
\hline \multirow{2}{*}{$\mathrm{C} 01$} & $234 \mathrm{C} 001$ & $0.0-0.33$ & 2.64 & 0.45 & $2.87(\mathrm{~J})$ & $1.53(\mathrm{~J})$ & 0.78 \\
\hline & $234 \mathrm{C} 002$ & $0.0-0.33$ & 2.24 & 0.41 & $2.66(\mathrm{~J})$ & $1.37(\mathrm{~J})$ & 0.85 \\
\hline $\mathrm{C} 02$ & $234 \mathrm{C} 003$ & $0.0-0.33$ & 2.5 & -- & $2.38(J)$ & $1.55(\mathrm{~J})$ & 0.95 \\
\hline $\mathrm{C03}$ & $234 \mathrm{C} 004$ & $0.0-0.5$ & 2.33 & -- & $2.58(\mathrm{~J})$ & $1.34(\mathrm{~J})$ & 0.73 \\
\hline $\mathrm{C04}$ & $234 \mathrm{C} 005$ & $0.0-0.5$ & 2.21 & 0.3 & $2.58(\mathrm{~J})$ & $1.28(\mathrm{~J})$ & 0.79 \\
\hline C05 & $234 \mathrm{C} 006$ & $0.0-0.5$ & 2.36 & 0.33 & $2.52(\mathrm{~J})$ & $1.26(\mathrm{~J})$ & 0.78 \\
\hline
\end{tabular}

aTaken from the generic guidelines for residual concentrations of actinium-228, bismuth-214, lead-212, lead-214, thallium-208, and thorium-232, as found in Chapter IV of DOE Order 5400.5, Change 2, "Radiation Protection of the Public and Environment."

(DOE, 1993). The PALs for these isotopes are specified as $5 \mathrm{pCi} / \mathrm{g}$ averaged over the first $15 \mathrm{~cm}$ of soil and $15 \mathrm{pCi} / \mathrm{g}$ for deeper soils (DOE, 1993). For purposes of this document, $15 \mathrm{~cm}$ is assumed to be equivalent to $0.5 \mathrm{ft}$ ( 6 inches); therefore, $5 \mathrm{pCi} / \mathrm{g}$ represents the PALs for these radionuclides in the surface soil ( 0 to $0.5 \mathrm{ft}$ depth).

${ }^{\mathrm{b}}$ Taken from the construction, commercial, industrial land use scenario in Table 2.1 of the NCRP Report No. 129, Recommended Screening Limits for Contaminated Surface Soil and Review Factors Relevant to Site-Specific Studies (NCRP, 1999). The values provided in this source document were scaled to a 25-millirem-per-year dose.

bgs $=$ Below ground surface

$\mathrm{cm}=$ Centimeter

DOE $=$ U.S. Department of Energy

$\mathrm{ft}=$ Foot

NCRP $=$ National Council on Radiation Protection and Measurements

$\mathrm{PAL}=$ Preliminary action level

$\mathrm{pCi} / \mathrm{g}=$ Picocuries per gram

$\mathrm{J}=$ Estimated value

$--=$ Not detected above minimum detectable concentrations 


\section{A.5.2.7 Plutonium, Strontium-90, and Uranium Isotopes}

Isotopic $\mathrm{Pu}$ and isotopic $\mathrm{U}$ analytical results for environmental samples collected at this CAS that were detected above MDCs are presented in Table A.5-7. No Sr-90 was identified above its MDC in any of the samples analyzed. No isotopic Pu or U sample results exceeded their respective PALs. Therefore, the FALs were established at the corresponding PAL concentrations.

Table A.5-7

Soil Sample Results for Isotopes Detected above Minimum Detectable Concentrations at CAS 12-09-01, Mud Pit

\begin{tabular}{|c|c|c|c|c|c|c|c|}
\hline \multirow[b]{2}{*}{$\begin{array}{c}\text { Sample } \\
\text { Location }\end{array}$} & \multirow[b]{2}{*}{$\begin{array}{l}\text { Sample } \\
\text { Number }\end{array}$} & \multirow[b]{2}{*}{$\begin{array}{c}\text { Depth } \\
\text { (ft bgs) }\end{array}$} & \multicolumn{5}{|c|}{ Contaminants of Potential Concern (pCi/g) } \\
\hline & & & 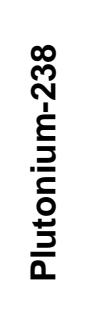 & 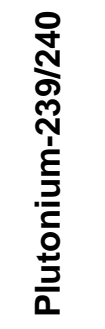 & 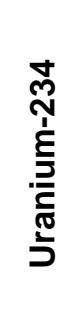 & 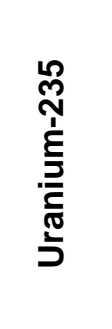 & 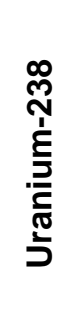 \\
\hline \multicolumn{3}{|c|}{ Final Action Levels ${ }^{a}$} & 13 & 12.7 & 143 & 17.6 & 105 \\
\hline \multirow{2}{*}{$\mathrm{C} 01$} & $234 \mathrm{C} 001$ & $0.0-0.33$ & -- & -- & 1.27 & 0.05 & 1.27 \\
\hline & $234 \mathrm{C} 002$ & $0.0-0.33$ & -- & -- & 1.13 & 0.056 & 1.19 \\
\hline $\mathrm{C} 02$ & $234 \mathrm{C} 003$ & $0.0-0.33$ & -- & -- & 1.08 & 0.08 & 1.15 \\
\hline $\mathrm{C} 03$ & $234 \mathrm{C} 004$ & $0.0-0.5$ & -- & 0.044 & 1.14 & 0.08 & 1.22 \\
\hline $\mathrm{C} 04$ & $234 \mathrm{C} 005$ & $0.0-0.5$ & 0.13 & 0.66 & 0.97 & 0.043 & 1.3 \\
\hline C05 & $234 \mathrm{C} 006$ & $0.0-0.5$ & 0.073 & 0.267 & 1.04 & 0.054 & 1.1 \\
\hline
\end{tabular}

${ }^{a}$ Taken from the construction, commercial, industrial land use scenario in Table 2.1 of the NCRP Report No. 129,

Recommended Screening Limits for Contaminated Surface Soil and Review Factors Relevant to Site-Specific Studies (NCRP, 1999). The values provided in this source document were scaled to a 25 -millirem-per-year dose.

bgs $=$ Below ground surface

$\mathrm{ft}=$ Foot

NCRP $=$ National Council on Radiation Protection and Measurements

$\mathrm{pCi} / \mathrm{g}=$ Picocuries per gram

$\mathrm{J}=$ Estimated value

$--=$ Not detected above minimum detectable concentrations 


\section{A.5.3 Nature and Extent of Contamination}

Based on the analytical results for soil samples collected within CAS 12-09-01, no COCs are present at this CAS.

\section{A.5.4 Revised Conceptual Site Model}

The results of the CAI at CAS 12-09-01 did not contradict the CSM. No revision of the CSM was necessary. 


\section{A.6.0 Corrective Action Site 12-09-08, Mud Pit}

Corrective Action Site 12-09-08 is located in the southwest corner of Area 12 of the NTS. This CAS consists of a drilling mud pit used for the construction of the U12e.14 HFR CH\#1 instrument hole.

The exact date of the construction of the mud pit is unknown; however, drilling of the instrument hole began on November 9, 1972. Two areas of potential release of contaminants are identified within the mud pit. The first is a length of metal piping that was protruding from the top of the berm wall by approximately $4 \mathrm{ft}$ and not connected at either end. The second potential release of contaminants was identified as a set of crushed 55-gal drums against the inner side of one of the berm walls (Figure A.6-1).

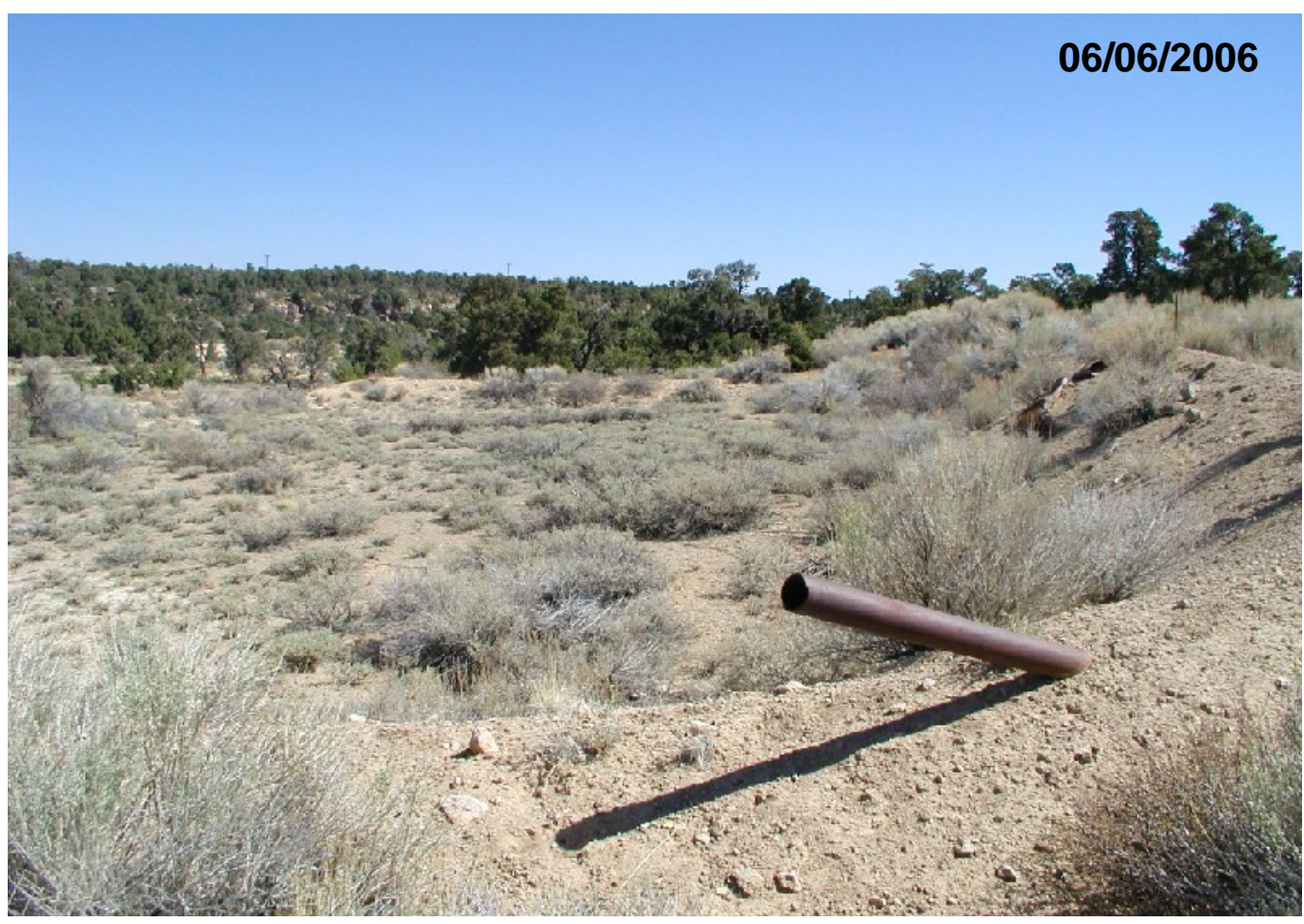

Figure A.6-1

Debris at CAS 12-09-08 


\section{A.6.1 Corrective Action Investigation}

A total of seven characterization samples (including one FD) were collected during investigation activities at CAS 12-09-08. The sample IDs, locations, types, and analyses are listed in Table A.6-1. The specific CAI activities conducted to satisfy the CAIP requirements at this CAS (NNSA/NSO, 2007) are described in the following sections.

Table A.6-1

Samples Collected at CAS 12-09-08, Mud Pit

\begin{tabular}{|c|c|c|c|c|c||}
\hline $\begin{array}{c}\text { Sample } \\
\text { Location }\end{array}$ & $\begin{array}{c}\text { Sample } \\
\text { Number }\end{array}$ & $\begin{array}{c}\text { Depth } \\
\text { (ft bgs) }\end{array}$ & Matrix & Purpose & Analyses \\
\hline \hline \multirow{2}{*}{ D01 } & $234 \mathrm{D} 001$ & $0.0-0.5$ & Soil & Environmental & Set 1 \\
\cline { 2 - 6 } & $234 \mathrm{D} 002$ & $0.5-1.0$ & Soil & Environmental & Set 1 \\
\hline \multirow{2}{*}{ D02 } & $234 \mathrm{D} 003$ & $0.0-0.5$ & Soil & Environmental & Set 1 \\
\cline { 2 - 6 } & $234 \mathrm{D} 004$ & $0.5-1.0$ & Soil & Environmental & Set 1 \\
\hline \multirow{3}{*}{ D03 } & 234D005 & $0.0-0.5$ & Soil & Environmental & Set 1 \\
\cline { 2 - 6 } & 234D006 & $0.0-0.5$ & Soil & Field Duplicate of 234D005 & Set 1 \\
\cline { 2 - 6 } & $234 \mathrm{D} 007$ & $1.0-1.5$ & Soil & Environmental & Set 1 \\
\hline N/A & 234D301 & N/A & Water & Trip Blank & VOCs only \\
\hline N/A & 234D302 & N/A & Water & Trip Blank & SeCs only 1 \\
\hline N/A & 234D303 & N/A & Water & Field Blank & Set \\
\hline
\end{tabular}

Set 1 = VOCs, SVOCs, RCRA Metals, TPH-DRO, PCBs, Gamma Spectroscopy, Isotopic Uranium, Isotopic Plutonium, Strontium-90

bgs $=$ Below ground surface $\mathrm{DRO}=$ Diesel-range organics $\mathrm{ft}=$ Foot

$\mathrm{N} / \mathrm{A}=$ Not applicable

$\mathrm{PCB}=$ Polychlorinated biphenyl
$\mathrm{RCRA}=$ Resource Conservation and Recovery Act SVOC = Semivolatile organic compound $\mathrm{TPH}=$ Total petroleum hydrocarbons $\mathrm{VOC}=$ Volatile organic compound

\section{A.6.1.1 Field Screening}

Decision I soil samples were field screened for alpha and beta/gamma radiation as specified in the CAU 234 CAIP (NNSA/NSO, 2007). The FSRs were compared to FSLs to guide subsequent sampling decisions where appropriate. Alpha and beta/gamma radiation FSLs were not exceeded during sampling activities. 


\section{A.6.1.2 Radiological Surveys}

An aerial radiological survey was performed in 1994 of Area 12, including CAS 12-09-08 (BN, 1999). The results of the survey were indistinguishable from background. As a result, no additional samples were collected (see Figure A.5-2).

\section{A.6.1.3 Deviations}

There were no deviations from the proposed sampling plan as described in the CAU 234 CAIP (NNSA/NSO, 2007).

\section{A.6.1.4 Visual Inspections}

Visual inspections of the CAS before sampling identified no additional features of concern. The locations of the pipe and crushed drums were verified. No visible staining was associated with the metal piping. There was no indication of staining associated with the discarded drums. Therefore, no additional samples were collected.

A walkover was conducted within the drilling mud sump during the collection of the biased samples from locations shown in Figure A.6-2 to identify additional sample locations based on biasing factors (i.e., staining). No additional biased sample locations were identified.

\section{A.6.1.5 Sample Collection}

Intrusive investigation activities (i.e., surface and shallow subsurface soil sampling) were conducted to support investigation activities. Soil samples were collected using scoops for surface samples and hand augers for subsurface samples.

\section{A.6.2 Investigation Results}

The following sections provide analytical results from the samples collected to complete investigation activities as outlined in the CAU 234 CAIP (NNSA/NSO, 2007). Investigation samples were analyzed for the CAIP-specified COPCs, which included VOCs, SVOCs, TPH-DRO, RCRA metals, gamma-emitting radionuclides, isotopic $\mathrm{U}$, isotopic $\mathrm{Pu}$, and $\mathrm{Sr}-90$. The PCBs are added parameters because these contaminants are a common concern at the NTS. The analytical parameters 


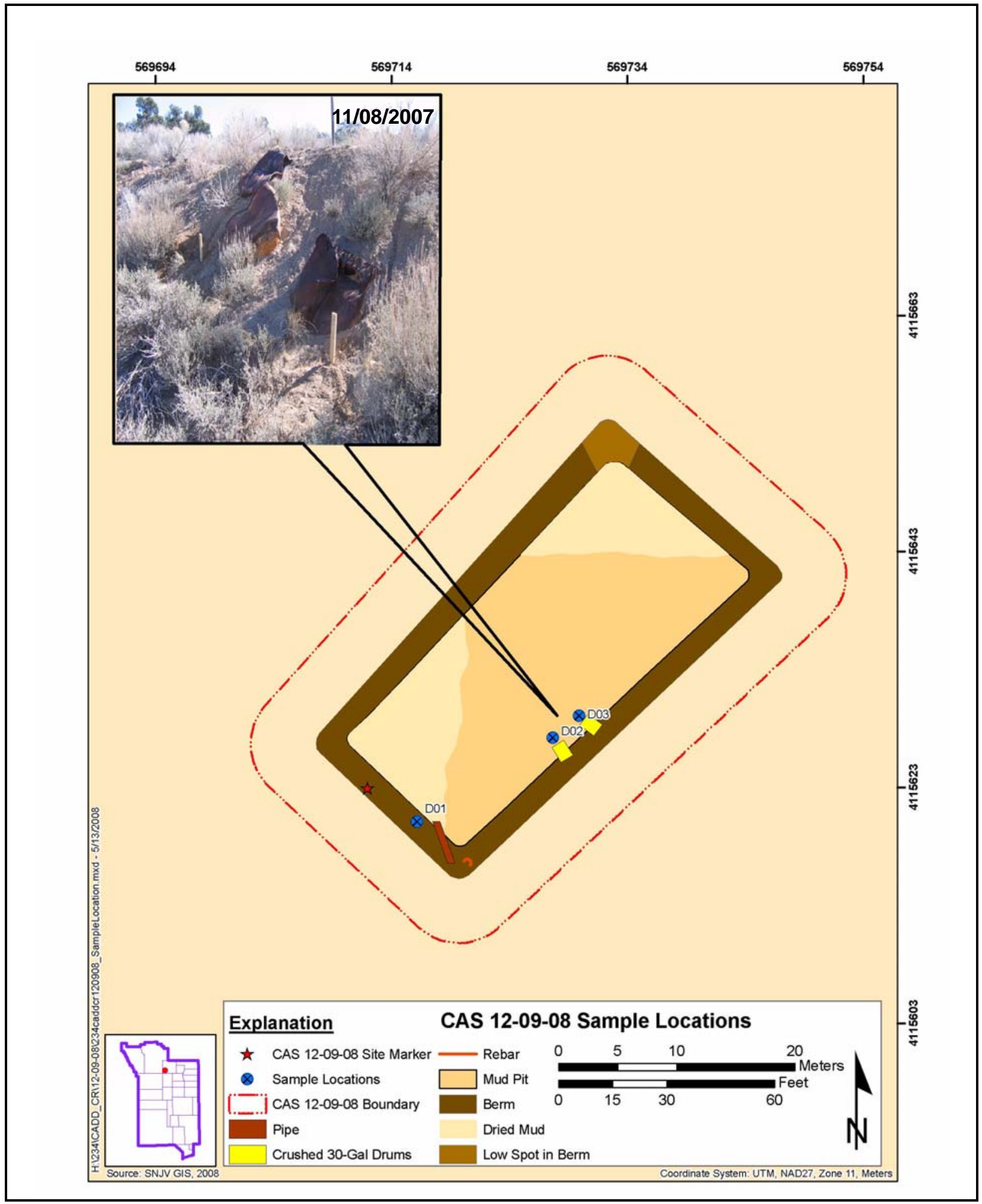

Figure A.6-2

Sample Locations for CAS 12-09-08 
and laboratory methods used to analyze the investigation samples are listed in Table A.2-2.

Table A.6-1 lists the sample-specific analytical suite for CAS 12-09-08.

Analytical results from the soil samples with concentrations exceeding MDCs are summarized in the following sections. An evaluation was conducted on all contaminants detected above MDCs by comparing individual concentration or activity results against the FALs. Establishment of the FALs is presented in Appendix D.

\section{A.6.2.1 Volatile Organic Compounds}

No VOC analytical results for environmental samples collected at this CAS were detected above their respective MDCs. Therefore, the FALs were established at the corresponding PAL concentrations.

\section{A.6.2.2 Semivolatile Organic Compounds}

The SVOCs analytical results for environmental samples collected at this CAS that were detected above MDCs are presented in Table A.6-2. None of the analyte concentrations exceeded their PALs. Therefore, the FALs were established at their corresponding PAL concentrations.

Table A.6-2

Soil Sample Results for SVOCs Detected above Minimum Detectable Concentrations at CAS 12-09-08, Mud Pit

\begin{tabular}{|c|c|c|c|}
\hline \multirow{2}{*}{$\begin{array}{c}\text { Sample } \\
\text { Location }\end{array}$} & $\begin{array}{c}\text { Sample } \\
\text { Number }\end{array}$ & $\begin{array}{c}\text { Depth } \\
\text { (ft bgs) }\end{array}$ & Contaminants of Potential Concern (mg/kg) \\
\cline { 3 - 4 } & & Bis(2-Ethylhexyl)Phthalate \\
\hline \multicolumn{3}{|c|}{ Final Action Levels } & $\mathbf{1 2 0}$ \\
\hline \hline D03 & 234 D007 & $1.0-1.5$ & $0.18(\mathrm{~J})$ \\
\hline
\end{tabular}

aBased on U.S. Environmental Protection Agency, Region 9 Preliminary Remediation Goals (PRGs) (EPA, 2004).

bgs $=$ Below ground surface

$\mathrm{ft}=$ Foot

$\mathrm{mg} / \mathrm{kg}=$ Milligrams per kilogram

$\mathrm{J}=$ Estimated value 


\section{A.6.2.3 Total Petroleum Hydrocarbons}

The TPH-DRO analytical results for soil samples collected at this CAS that were detected above MDCs are presented in Table A.6-3. None of the samples had TPH-DRO concentrations above the PAL of $100 \mathrm{mg} / \mathrm{kg}$. Therefore, the FAL was established at the PAL concentration.

Table A.6-3

\section{Soil Sample Results for TPH-DRO Detected above Minimum Detectable Concentrations at CAS 12-09-08, Mud Pit}

\begin{tabular}{|c|c|c|c|}
\hline \multirow{2}{*}{$\begin{array}{c}\text { Sample } \\
\text { Location }\end{array}$} & $\begin{array}{c}\text { Sample } \\
\text { Number }\end{array}$ & $\begin{array}{c}\text { Depth } \\
\text { (ft bgs) }\end{array}$ & Contaminants of Potential Concern (mg/kg) \\
\cline { 4 - 5 } & & Diesel-Range Organics \\
\hline \multicolumn{3}{|c|}{ Preliminary Action Levels ${ }^{\mathrm{a}}$} & $\mathbf{1 0 0}$ \\
\hline \hline D03 & $234 \mathrm{D007}$ & $1.0-1.5$ & 73 \\
\hline
\end{tabular}

aBased on Nevada Administrative Code, "Contamination of Soil: Establishment of Action Levels" (NAC, 2006).

bgs $=$ Below ground surface

$\mathrm{ft}=$ Foot

$\mathrm{mg} / \mathrm{kg}=$ Milligrams per kilogram

\section{A.6.2.4 RCRA Metals}

The RCRA metals analytical results for environmental samples collected at this CAS that were detected above MDCs are presented in Table A.6-4. No metals were detected at concentrations exceeding their PALs. Therefore, the FALs were established at the corresponding PAL concentrations.

\section{A.6.2.5 Polychlorinated Biphenyls}

Analytical results for soil samples collected at this CAS indicate that there are no PCBs detected above their respective MDCs. Therefore, the FALs were established at the PAL concentrations. 
Table A.6-4

\section{Soil Sample Results for RCRA Metals Detected above Minimum Detectable Concentrations at CAS 12-09-08, Mud Pit}

\begin{tabular}{|c|c|c|c|c|c|c|c|c|}
\hline \multirow{2}{*}{$\begin{array}{c}\text { Sample } \\
\text { Location }\end{array}$} & \multirow{2}{*}{$\begin{array}{l}\text { Sample } \\
\text { Number }\end{array}$} & \multirow{2}{*}{$\begin{array}{l}\text { Depth } \\
\text { (ft bgs) }\end{array}$} & \multicolumn{6}{|c|}{ Contaminants of Potential Concern $(\mathrm{mg} / \mathrm{kg})$} \\
\hline & & & Arsenic & Barium & Cadmium & Chromium & Lead & Selenium \\
\hline \multicolumn{3}{|c|}{ Final Action Levels } & $23^{a}$ & $67,000^{b}$ & $450^{b}$ & $450^{b}$ & $800^{b}$ & $5,100^{b}$ \\
\hline \multirow{2}{*}{ D01 } & $234 \mathrm{D} 001$ & $0.0-0.5$ & 4.5 & 160 & 0.12 & 7 & 10 & -- \\
\hline & 234D002 & $0.5-1.0$ & 4 & 160 & 0.065 & 6.5 & 10 & 0.46 \\
\hline \multirow{2}{*}{ D02 } & 234D003 & $0.0-0.5$ & 3.5 & 150 & 0.13 & 5.9 & 9.3 & -- \\
\hline & 234D004 & $0.5-1.0$ & 2.8 & 100 & 0.063 & 4 & 7.2 & -- \\
\hline \multirow{3}{*}{ D03 } & 234D005 & $0.0-0.5$ & 4.4 & 170 & 0.19 & 6.3 & 9.3 & -- \\
\hline & 234D006 & $0.0-0.5$ & 4 & 200 & 0.12 & 6 & 22 & -- \\
\hline & 234D007 & $1.0-1.5$ & 3.3 & 130 & 0.11 & 4.7 & 10 & -- \\
\hline
\end{tabular}

aBased on the background concentrations for metals. Background is considered the mean plus two times the standard deviation for sediment samples collected by the Nevada Bureau of Mines and Geology throughout the Nevada Test and Training Range (NBMG, 1998; Moore, 1999).

${ }^{\mathrm{b} B}$ Based on U.S. Environmental Protection Agency, Region 9 Preliminary Remediation Goals (PRGs) (EPA, 2004).

bgs $=$ Below ground surface

$\mathrm{ft}=$ Foot

$\mathrm{mg} / \mathrm{kg}=$ Milligrams per kilogram

$--=$ Not detected above minimum detectable concentrations

\section{A.6.2.6 Gamma-Emitting Radionuclides}

Gamma-emitting radionuclides analytical results for environmental samples collected at this CAS that were detected above MDCs are presented in Table A.6-5. None of the gamma-emitting radionuclides were found at concentrations exceeding their respective PALs. Therefore, the FALs were established at the corresponding PAL concentrations.

\section{A.6.2.7 Plutonium, Strontium-90, and Uranium Isotopes}

Isotopic $\mathrm{Pu}$ and isotopic $\mathrm{U}$ analytical results for environmental samples collected at this $\mathrm{CAS}$ that were detected above MDCs are presented in Table A.6-6. No Sr-90 was identified above its MDC in any of the samples analyzed. No isotopic Pu or U exceeded the PALs. Therefore, the FALs were established at the corresponding PAL concentrations. 
Table A.6-5

\section{Soil Sample Results for Gamma-Emitting Radionuclides Detected above Minimum Detectable Concentrations at CAS 12-09-08, Mud Pit}

\begin{tabular}{|c|c|c|c|c|c|c|c|c|c|c|}
\hline \multirow{2}{*}{$\begin{array}{c}\text { Sample } \\
\text { Location }\end{array}$} & \multirow{2}{*}{$\begin{array}{c}\text { Sample } \\
\text { Number }\end{array}$} & \multirow{3}{*}{$\begin{array}{l}\text { Depth } \\
\text { (ft bgs) }\end{array}$} & \multicolumn{8}{|c|}{ Contaminants of Potential Concern $(\mathrm{pCi} / \mathrm{g})$} \\
\hline & & & \multicolumn{2}{|c|}{ Actinium-228 } & \multicolumn{2}{|c|}{ Lead-212 } & \multicolumn{2}{|c|}{ Lead-214 } & \multicolumn{2}{|c|}{ Thallium-208 } \\
\hline \multicolumn{2}{|c|}{ Final Action Levels ${ }^{a}$} & & 5 & 15 & 5 & 15 & 5 & 15 & 5 & 15 \\
\hline \multirow{2}{*}{ D01 } & 234D001 & $0.0-0.5$ & 2.56 & -- & $2.77(\mathrm{~J})$ & -- & $1.24(\mathrm{~J})$ & -- & 0.84 & -- \\
\hline & 234D002 & $0.5-1.0$ & -- & 2.23 & -- & $2.65(J)$ & -- & $1.1(\mathrm{~J})$ & -- & 0.89 \\
\hline \multirow{2}{*}{ D02 } & 234D003 & $0.0-0.5$ & 2.16 & -- & $2.62(\mathrm{~J})$ & -- & $1.14(\mathrm{~J})$ & -- & 0.66 & \\
\hline & 234D004 & $0.5-1.0$ & -- & 2.14 & -- & $2.54(\mathrm{~J})$ & -- & $0.95(J)$ & -- & 0.71 \\
\hline \multirow{3}{*}{ D03 } & 234D005 & $0.0-0.5$ & 2.14 & -- & $2.75(\mathrm{~J})$ & -- & $1.18(\mathrm{~J})$ & -- & 0.69 & -- \\
\hline & 234D006 & $0.0-0.5$ & 2.12 & -- & $2.48(\mathrm{~J})$ & -- & $1.25(\mathrm{~J})$ & -- & 0.81 & -- \\
\hline & 234D007 & $1.0-1.5$ & -- & 2.19 & -- & $2.91(\mathrm{~J})$ & -- & $1.26(\mathrm{~J})$ & -- & 0.86 \\
\hline
\end{tabular}

aTaken from the generic guidelines for residual concentrations of actinium-228, bismuth-214, lead-212, lead-214, thallium-208, and thorium-232, as found in Chapter IV of DOE Order 5400.5, Change 2, "Radiation Protection of the Public and Environment."

(DOE, 1993). The PALs for these isotopes are specified as $5 \mathrm{pCi} / \mathrm{g}$ averaged over the first $15 \mathrm{~cm}$ of soil and $15 \mathrm{pCi} / \mathrm{g}$ for deeper soils (DOE, 1993). For purposes of this document, $15 \mathrm{~cm}$ is assumed to be equivalent to $0.5 \mathrm{ft}$ ( 6 inches); therefore, $5 \mathrm{pCi} / \mathrm{g}$ represents the PALs for these radionuclides in the surface soil ( 0 to $0.5 \mathrm{ft}$ depth).

bgs $=$ Below ground surface

$\mathrm{cm}=$ Centimeter

DOE $=$ U.S. Department of Energy

$\mathrm{ft}=$ Foot

$\mathrm{PAL}=$ Preliminary action level

$\mathrm{pCi} / \mathrm{g}=$ Picocuries per gram

$\mathrm{J}=$ Estimated value

$--=$ Not detected above minimum detectable concentrations

Table A.6-6

Soil Sample Results for Isotopes Detected above Minimum Detectable Concentrations at CAS 12-09-08, Mud Pit

(Page 1 of 2 )

\begin{tabular}{|c|c|c|c|c|c|c||}
\hline \multirow{2}{*}{$\begin{array}{c}\text { Sample } \\
\text { Location }\end{array}$} & \multirow{2}{*}{$\begin{array}{c}\text { Sample } \\
\text { Number }\end{array}$} & \multirow{2}{*}{$\begin{array}{c}\text { Depth } \\
\text { (ft bgs) }\end{array}$} & \multicolumn{4}{|c|}{ Contaminants of Potential Concern (pCi/g) } \\
\cline { 5 - 8 } & & & Plutonium-239/240 & Uranium-234 & Uranium-235 & Uranium-238 \\
\hline \multicolumn{2}{|c|}{ Final Action Levels ${ }^{\text {a }}$} & $\mathbf{1 2 . 7}$ & $\mathbf{1 4 3}$ & $\mathbf{1 7 . 6}$ & $\mathbf{1 0 5}$ \\
\hline \hline \multirow{2}{*}{ D01 } & 234D001 & $0.0-0.5$ & -- & 0.96 & 0.047 & 0.96 \\
\cline { 2 - 7 } & 234D002 & $0.5-1.0$ & -- & 0.96 & -- & 0.84 \\
\hline \multirow{2}{*}{ D02 } & 234D003 & $0.0-0.5$ & -- & 1.06 & -- & 1.09 \\
\cline { 2 - 7 } & 234D004 & $0.5-1.0$ & -- & 0.99 & -- & 1.09 \\
\hline
\end{tabular}




\section{Table A.6-6 \\ Soil Sample Results for Isotopes Detected above Minimum Detectable Concentrations at CAS 12-09-08, Mud Pit}

(Page 2 of 2)

\begin{tabular}{||c|c|c|c|c|c|c||}
\hline \multirow{2}{*}{$\begin{array}{c}\text { Sample } \\
\text { Location }\end{array}$} & \multirow{2}{*}{$\begin{array}{c}\text { Sample } \\
\text { Number }\end{array}$} & $\begin{array}{c}\text { Depth } \\
\text { (ft bgs) }\end{array}$ & \multicolumn{4}{|c|}{ Contaminants of Potential Concern (pCi/g) } \\
\cline { 4 - 7 } & & Plutonium-239/240 & Uranium-234 & Uranium-235 & Uranium-238 \\
\hline \multicolumn{3}{||c|}{ Final Action Levels ${ }^{\mathbf{a}}$} & $\mathbf{1 2 . 7}$ & $\mathbf{1 4 3}$ & $\mathbf{1 7 . 6}$ & $\mathbf{1 0 5}$ \\
\hline \hline \multirow{3}{*}{ D03 } & $234 \mathrm{D} 005$ & $0.0-0.5$ & 0.028 & 1.03 & 0.081 & 1.03 \\
\cline { 2 - 7 } & 234D006 & $0.0-0.5$ & -- & 0.91 & 0.053 & 0.92 \\
\cline { 2 - 7 } & 234D007 & $1.0-1.5$ & -- & 0.9 & 0.076 & 1.05 \\
\hline
\end{tabular}

Taken from the construction, commercial, industrial land use scenario in Table 2.1 of the NCRP Report No. 129, Recommended Screening Limits for Contaminated Surface Soil and Review Factors Relevant to Site-Specific Studies (NCRP, 1999). The values provided in this source document were scaled to a 25-millirem-per-year dose.

bgs = Below ground surface

$\mathrm{ft}=$ Foot

NCRP $=$ National Council on Radiation Protection and Measurements

$\mathrm{pCi} / \mathrm{g}=$ Picocuries per gram

$--=$ Not detected above minimum detectable concentrations

\section{A.6.3 Nature and Extent of Contamination}

Based on the analytical results for soil samples collected within CAS 12-09-08, no COCs are present.

\section{A.6.4 Revised Conceptual Site Model}

The results of the CAI at CAS 12-09-08 did not contradict the CSM. No revision of the CSM was necessary. 


\section{A.7.0 Corrective Action Site 12-30-14, Cellar}

Corrective Action Site 12-30-14 is located in the southwest corner of Area 12 of the NTS. This CAS consists of a cellar that was the site for the drilling of the U12r PS\#1A and U12r PS\#1AS post-test boreholes. U12r PS\#1A (2,045 ft bgs) was drilled from January 19 to 23, 1969, and U12r PS\#1AS $(2,007 \mathrm{ft}$ bgs $)$ was drilled on January 23 and 24, 1969. The U12r Wineskin test took place on January 15, 1969. The open top cellar is approximately $9 \mathrm{ft}$ deep and $10 \mathrm{ft}$ in diameter. The inner wall of the cellar is lined with corrugated metal. Liquid can commonly be found within the cellar and has varied from approximately $7 \mathrm{ft}$ deep to $1.5 \mathrm{ft}$ deep during the site investigation, spanning approximately two years (Figure A.7-1).

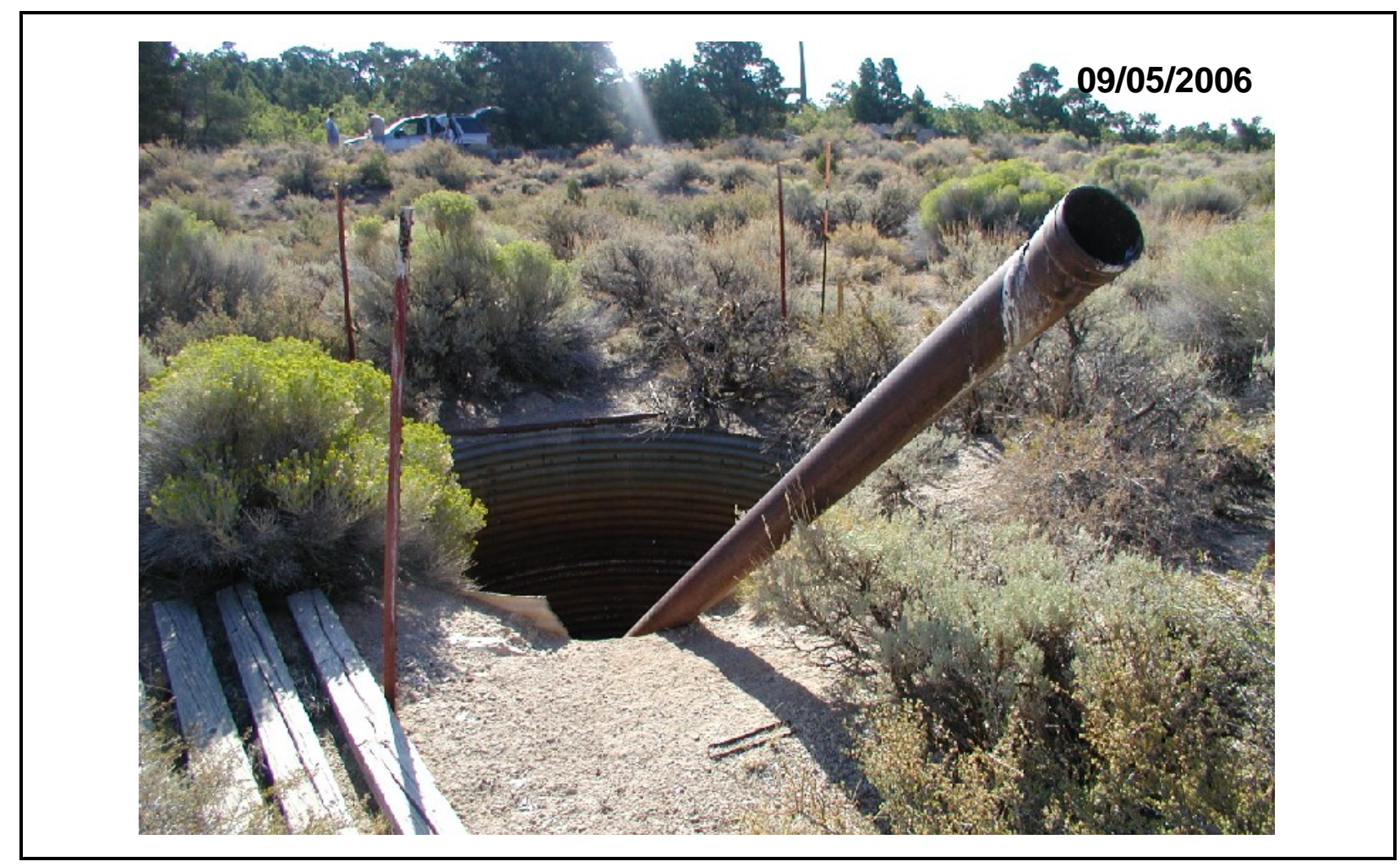

Figure A.7-1

Cellar at CAS 12-30-14 


\section{A.7.1 Corrective Action Investigation}

A total of four PSM samples (including one FD) were collected during investigation activities at CAS 12-30-14. The sample IDs, locations, types, and analyses are listed in Table A.7-1. The specific CAI activities conducted to satisfy the CAIP requirements at this CAS (NNSA/NSO, 2007) are described in the following sections.

Table A.7-1

Samples Collected at CAS 12-30-14, Cellar

\begin{tabular}{|c|c|c|c|c|c||}
\hline $\begin{array}{c}\text { Sample } \\
\text { Location }\end{array}$ & $\begin{array}{c}\text { Sample } \\
\text { Number }\end{array}$ & $\begin{array}{c}\text { Thickness } \\
\text { (ft) }\end{array}$ & Matrix & Purpose & Analyses \\
\hline \hline E-Cellar & $234 \mathrm{E} 001$ & $1.5^{\mathrm{a}}$ & Liquid & Environmental & Set 1 \\
\hline E-Sediment & $234 \mathrm{E} 002$ & $1.0^{\mathrm{b}}$ & Sediment & Environmental & Set 1 \\
\hline \multirow{2}{*}{ E-Sediment } & $234 \mathrm{E} 003$ & $1.0^{\mathrm{b}}$ & Sediment & Environmental & Set 1 \\
\cline { 2 - 6 } & $234 \mathrm{E} 004$ & $1.0^{\mathrm{b}}$ & Sediment & Field Duplicate of 234E003 & Set 1 \\
\hline N/A & $234 \mathrm{E} 301$ & $\mathrm{~N} / \mathrm{A}$ & Water & Trip Blank & VOCs only \\
\hline $\mathrm{N} / \mathrm{A}$ & $234 \mathrm{E} 302$ & $\mathrm{~N} / \mathrm{A}$ & Water & Field Blank & Set 1 \\
\hline $\mathrm{N} / \mathrm{A}$ & $234 \mathrm{E} 303$ & $\mathrm{~N} / \mathrm{A}$ & Water & Trip Blank & VOCs only \\
\hline
\end{tabular}

Set 1 = VOCs, SVOCs, RCRA Metals, TPH-DRO, PCBs, Gamma Spectroscopy, Isotopic Uranium, Isotopic Plutonium, Strontium-90

${ }^{a}$ Thickness of water column above sediment on cellar floor.

${ }^{\mathrm{b}}$ Thickness of sediment on cellar floor beneath water column.

RCRA = Resource Conservation and Recovery Act

$\mathrm{DRO}=$ Diesel-range organics

SVOC = Semivolatile organic compound

$\mathrm{ft}=\mathrm{Foot}$

$\mathrm{TPH}=$ Total petroleum hydrocarbons

$\mathrm{N} / \mathrm{A}=$ Not applicable

$\mathrm{VOC}=$ Volatile organic compound

$\mathrm{PCB}=$ Polychlorinated biphenyl

\section{A.7.1.1 Field Screening}

Samples were screened for gamma-emitting radionuclides using a gamma spectrometer at Building 23-153 due to the wet nature of the samples to compare to shipping requirements. The results were at background concentrations; therefore, no special packaging was required. 


\section{A.7.1.2 Radiological Surveys}

An aerial radiological survey was conducted in 1994 of Area 12, including CAS 12-30-14 (BN, 1999). The findings of the survey were indistinguishable from background. Therefore, no additional samples were collected (see Figure A.5-2).

\section{A.7.1.3 Visual Inspections}

Other than the PSM located within the cellar, no additional locations were identified that required sample collection. Therefore, no additional samples were collected.

\section{A.7.1.4 Sample Collection}

Sampling activities at CAS 12-30-14 included the collection of environmental liquid and sediment samples from both the liquid and sediment phases identified in Figure A.7-2. Liquid samples were collected using a scoop on a pole. Scoops were used for the collection of sediment from the cellar base, then transferred to aluminum pans for processing.

\section{A.7.1.5 Deviations}

There were no deviations to the CAIP requirements (NNSA/NSO, 2007) for sampling at this CAS.

\section{A.7.2 Investigation Results}

The following sections provide analytical results from the samples collected to complete investigation activities as outlined in the CAIP (NNSA/NSO, 2007). Investigation samples were analyzed for the CAIP-specified parameters, which included VOCs, SVOCs, TPH-DRO, RCRA metals, gamma-emitting radionuclides, isotopic $\mathrm{U}$, isotopic $\mathrm{Pu}$, and $\mathrm{Sr}-90$. The PCBs are added parameters because these contaminants are a common concern at the NTS. The analytical parameters and laboratory methods used to analyze the investigation samples are listed in Table A.2-2. Table A.7-1 lists the sample-specific analytical suite for CAS 12-30-14.

Analytical results from the PSM samples with concentrations exceeding MDCs are summarized in the following sections. An evaluation was conducted on all contaminants detected above MDCs by 


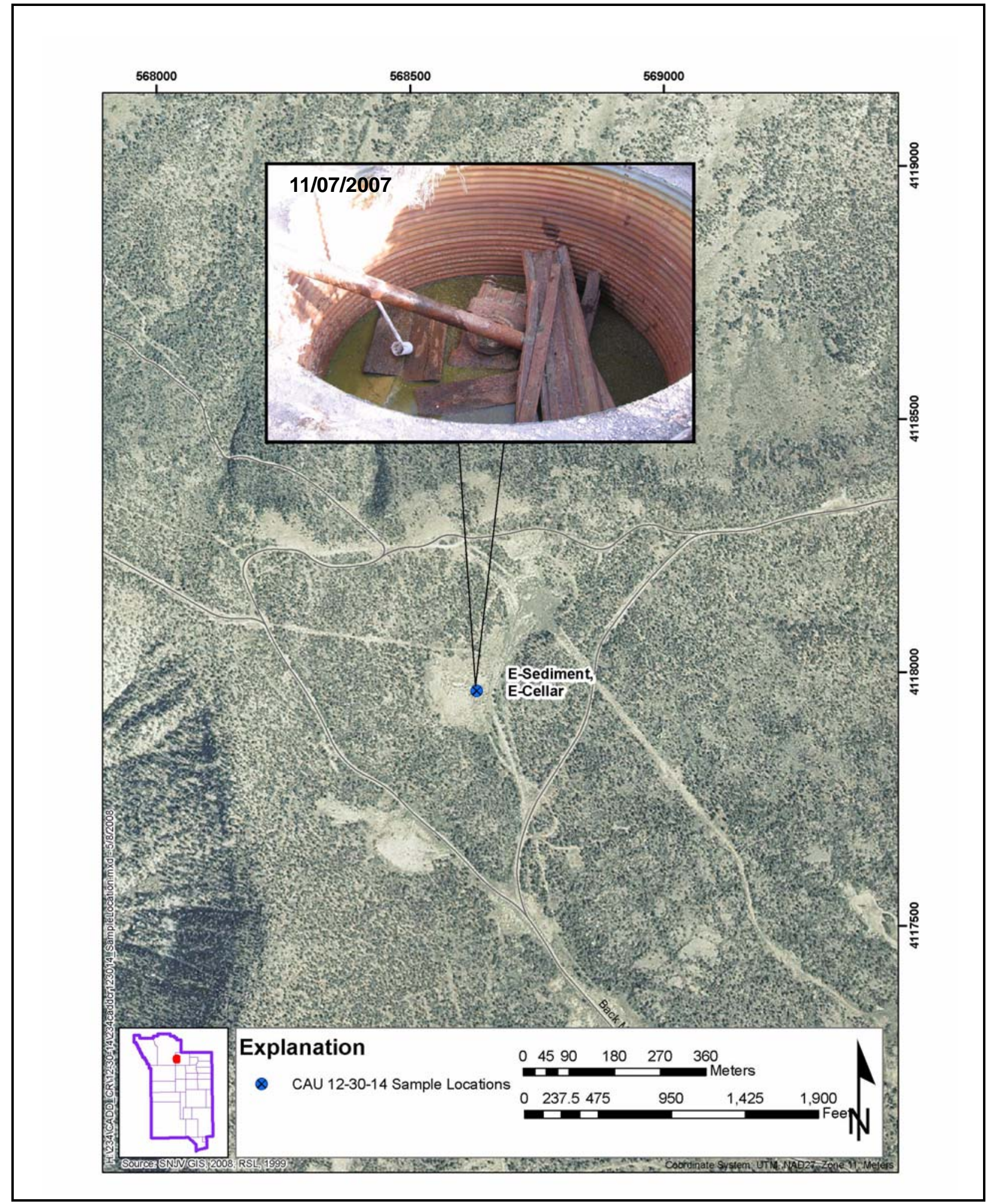

Figure A.7-2

Sample Locations for CAS 12-30-14 
comparing individual concentration or activity results against the PSM criteria established in the CAIP (NNSA/NSO, 2007).

\section{A.7.2.1 Volatile Organic Compounds}

The VOCs analytical results for PSM samples collected at this CAS that were detected above MDCs are presented in Tables A.7-2 and A.7-3. Table A.7-2 lists the contaminants identified above their respective MDCs in the sediment contents of the cellar. Table A.7-3 lists the contaminants identified above their respective MDCs in the liquid contents of the cellar. No VOCs were detected at concentrations exceeding their respective PSM criteria in either phase.

Table A.7-2

Sediment PSM Sample Results for VOCs Detected above Minimum Detectable Concentrations at CAS 12-30-14, Cellar

\begin{tabular}{||c|c|c|c|c||}
\hline \multirow{2}{*}{$\begin{array}{c}\text { Sample } \\
\text { Location }\end{array}$} & \multirow{2}{*}{$\begin{array}{c}\text { Sample } \\
\text { Number }\end{array}$} & \multirow{2}{*}{$\begin{array}{c}\text { Thickness } \\
\text { (ft) }\end{array}$} & \multicolumn{2}{c|}{ Contaminants of Potential Concern (mg/kg) } \\
\cline { 4 - 5 } & & & Acetone & p-Isopropyltoluene \\
\hline \multicolumn{3}{|c|}{ PSM Criteria $^{\mathrm{a}}$} & $\mathbf{5 4 , 0 0 0}$ & $\mathbf{2 , 0 0 0}$ \\
\hline \hline E-Sediment & $234 \mathrm{E} 002$ & $1.0^{\mathrm{b}}$ & 0.059 & 0.16 \\
\hline E-Sediment & $234 \mathrm{E} 003$ & $1.0^{\mathrm{b}}$ & $0.012(\mathrm{~J})$ & 0.030 \\
\hline E-Sediment & $234 \mathrm{E} 004$ & $1.0^{\mathrm{b}}$ & -- & 0.049 \\
\hline
\end{tabular}

aBased on U.S. Environmental Protection Agency Region 9 Preliminary Remediation Goals (PRGs) (EPA, 2004).

bThickness of sediment on cellar floor.

$\mathrm{ft}=$ Foot

$\mathrm{mg} / \mathrm{kg}=$ Milligrams per kilogram

PSM $=$ Potential source material

$\mathrm{J}=$ Estimated value

-- = Not detected above minimum detectable concentrations

\section{A.7.2.2 Semivolatile Organic Compounds}

The SVOCs analytical results for PSM samples collected in the sediment contents of this CAS that were detected above MDCs are presented in Table A.7-4. No SVOCs were detected in the liquid contents of the cellar. No SVOCs were detected at concentrations exceeding the PSM criteria. 
Table A.7-3

Liquid PSM Sample Results for VOCs Detected above Minimum Detectable Concentrations at CAS 12-30-14, Cellar

\begin{tabular}{|c|c|c|c|}
\hline \multirow{2}{*}{$\begin{array}{c}\text { Sample } \\
\text { Location }\end{array}$} & \multirow{2}{*}{$\begin{array}{c}\text { Sample } \\
\text { Number }\end{array}$} & \multirow{2}{*}{$\begin{array}{c}\text { Thickness } \\
\text { (ft) }\end{array}$} & Contaminants of Potential Concern (mg/L) \\
\hline & & & Acetone \\
\hline \multicolumn{3}{|c|}{ PSM Criteria $^{a}$} & None \\
\hline E-Cellar & 234E001 & $1.5^{\mathrm{b}}$ & 0.064 \\
\hline
\end{tabular}

"Based on Code of Federal Regulations, Title 40 CFR Part 261, "Identification and Listing of Hazardous Waste" (CFR, 2006).

${ }^{\mathrm{b}}$ Thickness of water column above sediment on cellar floor.

$\mathrm{ft}=$ Foot

$\mathrm{mg} / \mathrm{L}=$ Milligrams per liter

$\mathrm{PSM}=$ Potential source material

Table A.7-4

Sediment PSM Sample Results for SVOCs Detected above Minimum Detectable Concentrations at CAS 12-30-14, Cellar

\begin{tabular}{|c|c|c|c||}
\hline \multirow{2}{*}{$\begin{array}{c}\text { Sample } \\
\text { Location }\end{array}$} & \multirow{2}{*}{$\begin{array}{c}\text { Sample } \\
\text { Number }\end{array}$} & $\begin{array}{c}\text { Thickness } \\
(\mathrm{ft})\end{array}$ & Contaminants of Potential Concern (mg/kg) \\
\cline { 3 - 4 } & & Bis(2-Ethylhexyl)Phthalate \\
\hline \multicolumn{3}{|c|}{ PSM Criteria $^{\mathrm{a}}$} & $\mathbf{1 2 0}$ \\
\hline \hline E-Sediment & $234 \mathrm{E} 004$ & $1.0^{\mathrm{b}}$ & 0.640 \\
\hline
\end{tabular}

âThickness on U.S. Environmental Protection Agency Region 9 Preliminary Remediation Goals (PRGs) (EPA, 2004).

${ }^{\mathrm{b}}$ Thickness of water column above sediment on cellar floor.

$\mathrm{ft}=$ Foot

$\mathrm{mg} / \mathrm{kg}=$ Milligrams per kilogram

$\mathrm{PSM}=$ Potential source material

\section{A.7.2.3 Total Petroleum Hydrocarbons}

The TPH-DRO analytical results for environmental samples collected in the sediment at this CAS that were detected above MDCs are presented in Table A.7-5. No TPH-DRO results exceeded the PSM criteria of $100 \mathrm{mg} / \mathrm{kg}$. No TPH-DRO was identified in samples collected from the liquid contents of the cellar. 
Table A.7-5

Sediment PSM Sample Results for TPH-DRO Detected above Minimum Detectable Concentrations at CAS 12-30-14, Cellar

\begin{tabular}{||c|c|c|c||}
\hline \multirow{2}{*}{$\begin{array}{c}\text { Sample } \\
\text { Location }\end{array}$} & \multirow{2}{*}{$\begin{array}{c}\text { Sample } \\
\text { Number }\end{array}$} & \multirow{2}{*}{$\begin{array}{c}\text { Thickness } \\
(\mathrm{ft})\end{array}$} & Contaminants of Potential Concern (mg/kg) \\
\cline { 4 - 4 } & & & $\mathbf{1 0 0}$ \\
\hline \multicolumn{3}{|c|}{ PSM Criteria $^{\mathrm{a}}$} & 60 \\
\hline \hline E-Sediment & $234 \mathrm{E} 002$ & $1.0^{\mathrm{b}}$ & 35 \\
\hline E-Sediment & $234 \mathrm{E} 003$ & $1.0^{\mathrm{b}}$ & 49 \\
\hline E-Sediment & $234 \mathrm{E} 004$ & $1.0^{\mathrm{b}}$ & Diesel-Range Organics \\
\hline
\end{tabular}

aBased on Nevada Administrative Code, "Contamination of Soil: Establishment of Action Levels" (NAC, 2006).

${ }^{\text {b} T h i c k n e s s ~ o f ~ s e d i m e n t ~ o n ~ c e l l a r ~ f l o o r ~ b e n e a t h ~ w a t e r ~ c o l u m n . ~}$

$\mathrm{ft}=$ Foot

$\mathrm{mg} / \mathrm{kg}=$ Milligrams per kilogram

$\mathrm{PSM}=$ Potential source material

\section{A.7.2.4 RCRA Metals}

The RCRA metals analytical results for PSM samples collected from the sediment portion of this CAS that were detected above MDCs are presented in Table A.7-6. The RCRA metals analytical results for PSM samples collected from the liquid portion of this CAS that were detected above MDCs are presented in Table A.7-7. No metals were detected at concentrations exceeding the PSM criteria in either phase.

\section{A.7.2.5 Polychlorinated Biphenyls}

Analytical results for PSM samples collected at this CAS indicate that there are no PCBs detected above their respective MDCs.

\section{A.7.2.6 Gamma-Emitting Radionuclides}

Gamma-emitting radionuclides analytical results for PSM samples collected at this CAS that were detected above MDCs are presented in Table A.7-8. No gamma-emitting radionuclide exceeded PSM criteria. 
Table A.7-6

Sediment PSM Sample Results for RCRA Metals Detected above
Minimum Detectable Concentrations at CAS 12-30-14, Cellar

\begin{tabular}{|c|c|c|c|c|c|c|c|c|c|}
\hline \multirow[b]{2}{*}{$\begin{array}{l}\text { Sample } \\
\text { Location }\end{array}$} & \multirow[b]{2}{*}{$\begin{array}{l}\text { Sample } \\
\text { Number }\end{array}$} & \multirow[b]{2}{*}{$\begin{array}{c}\text { Thickness } \\
\text { (ft) }\end{array}$} & \multicolumn{7}{|c|}{ Contaminants of Potential Concern $(\mathrm{mg} / \mathrm{kg})$} \\
\hline & & & 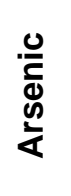 & 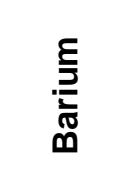 & $\frac{\varepsilon}{\bar{g}}$ & 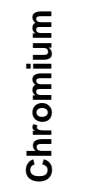 & ఫ్త & $\frac{\text { E }}{\stackrel{D}{\frac{D}{\omega}}}$ & $\frac{\bar{\Xi}}{\overline{\bar{\omega}}}$ \\
\hline \multicolumn{3}{|c|}{ PSM Criteria } & $23^{a}$ & $67,000^{b}$ & $450^{b}$ & $450^{b}$ & $800^{b}$ & $5,100^{b}$ & $5,100^{b}$ \\
\hline E-Sediment & 234E002 & $1.0^{\mathrm{C}}$ & 3.1 & 3,100 & 1 & 6.3 & 210 & -- & 0.71 \\
\hline E-Sediment & 234E003 & $1.0^{c}$ & 4.1 & 130 & -- & 5.6 & 16 & 0.57 & -- \\
\hline E-Sediment & 234E004 & $1.0^{c}$ & 5 & 150 & 0.84 & 5 & 23 & 0.62 & -- \\
\hline
\end{tabular}

${ }^{\mathrm{a}}$ Based on the background concentrations for metals. Background is considered the mean plus two times the standard deviation for sediment samples collected by the Nevada Bureau of Mines and Geology throughout the Nevada Test and Training Range (NBMG, 1998; Moore, 1999).

${ }^{b}$ Based on U.S. Environmental Protection Agency Region 9 Preliminary Remediation Goals (PRGs) (EPA, 2004).

${ }^{\mathrm{C}}$ Thickness of sediment on cellar floor beneath water column.

$\mathrm{ft}=$ Foot

$\mathrm{mg} / \mathrm{kg}=$ Milligrams per kilogram

PSM = Potential source material

-- = Not detected above minimum detectable concentrations

Table A.7-7

Liquid PSM Sample Results for RCRA Metals Detected above Minimum Detectable Concentrations at CAS 12-30-14, Cellar

\begin{tabular}{|c|c|c|c|c|c|}
\hline \multirow{2}{*}{$\begin{array}{l}\text { Sample } \\
\text { Location }\end{array}$} & \multirow{2}{*}{$\begin{array}{l}\text { Sample } \\
\text { Number }\end{array}$} & \multirow{2}{*}{$\begin{array}{l}\text { Thickness } \\
\text { (ft) }\end{array}$} & \multicolumn{3}{|c|}{ Contaminants of Potential Concern (mg/L) } \\
\hline & & & Arsenic & Barium & Lead \\
\hline \multicolumn{3}{|c|}{ PSM Criteria $^{a}$} & 5.0 & 100.0 & 5.0 \\
\hline E-Cellar & 234E001 & $1.5^{\mathrm{b}}$ & 0.0082 & 0.15 & 0.002 \\
\hline
\end{tabular}

aBased on Code of Federal Regulations, Title 40 CFR Part 261, "Identification and Listing of Hazardous Waste" (CFR, 2006).

${ }^{\mathrm{b}}$ Thickness of water column above sediment on cellar floor.

$\mathrm{ft}=$ Foot

$\mathrm{mg} / \mathrm{L}=$ Milligrams per liter

PSM $=$ Potential source material 
Table A.7-8

Sediment PSM Sample Results for Gamma-Emitting Radionuclides Detected above Minimum Detectable Concentrations at 12-30-14, Cellar

\begin{tabular}{||c|c|c|c|c|c|c||}
\hline \multirow{2}{*}{$\begin{array}{c}\text { Sample } \\
\text { Location }\end{array}$} & \multirow{2}{*}{$\begin{array}{c}\text { Sample } \\
\text { Number }\end{array}$} & \multirow{2}{*}{$\begin{array}{c}\text { Thickness } \\
(\mathrm{ft})\end{array}$} & \multicolumn{4}{|c|}{ Contaminants of Potential Concern (pCi/g) } \\
\cline { 5 - 7 } & & & Actinium-228 & Lead-212 & Lead-214 & Thallium-208 \\
\hline \multicolumn{3}{|c|}{ PSM Criteria $^{\mathrm{a}}$} & $\mathbf{5}$ & $\mathbf{5}$ & $\mathbf{5}$ & $\mathbf{5}$ \\
\hline \hline E-Sediment & $234 \mathrm{E} 002$ & $1.0^{\mathrm{b}}$ & 1.83 & $2.19(\mathrm{~J})$ & $1.48(\mathrm{~J})$ & 0.66 \\
\hline E-Sediment & $234 \mathrm{E} 003$ & $1.0^{\mathrm{b}}$ & 1.72 & $1.67(\mathrm{~J})$ & $1.29(\mathrm{~J})$ & 0.56 \\
\hline E-Sediment & $234 \mathrm{E} 004$ & $1.0^{\mathrm{b}}$ & 1.53 & $1.61(\mathrm{~J})$ & $1.01(\mathrm{~J})$ & 0.47 \\
\hline
\end{tabular}

Taken from the generic guidelines for residual concentrations of actinium-228, bismuth-214, lead-212, lead-214, thallium-208, and thorium-232, as found in Chapter IV of DOE Order 5400.5, Change 2, Radiation Protection of the Public and Environment (DOE, 1993). The PALs for these isotopes is specified as $5 \mathrm{pCi} / \mathrm{g}$ averaged over the first $15 \mathrm{~cm}$ of soil and $15 \mathrm{pCi} / \mathrm{g}$ for deeper soils (DOE, 1993). For purposes of this document, $15 \mathrm{~cm}$ is assumed to be equivalent to $0.5 \mathrm{ft}$ ( 6 inches); therefore, $5 \mathrm{pCi} / \mathrm{g}$ represents the PALs for these radionuclides in the surface soil ( 0 to $0.5 \mathrm{ft}$ depth).

${ }^{\mathrm{b}}$ Thickness of sediment on cellar floor beneath water column.

$\mathrm{cm}=$ Centimeter

DOE $=$ U.S. Department of Energy

$\mathrm{ft}=$ Foot

$\mathrm{PAL}=$ Preliminary action level

$\mathrm{pCi} / \mathrm{g}=$ Picocuries per gram

PSM $=$ Potential source material

$\mathrm{J}=$ Estimated value

\section{A.7.2.7 Plutonium, Strontium-90, and Uranium Isotopes}

The Sr-90 and isotopic U analytical results for PSM sediment samples collected at this CAS that were detected above MDCs are presented in Table A.7-9. No isotopic Pu was detected in any of the samples analyzed. No Sr-90 or isotopic U exceeded PSM criteria in either phase. The only result above MDCs for the liquid sample 234E001 was Sr-90 at 3.05 picocuries per liter (pCi/L). There is no radiological PSM criteria for comparison to the liquid sample.

\section{A.7.3 Nature and Extent of Contamination}

Based on the analytical results for the PSM samples collected within CAS 12-30-14, no PSM was identified at this CAS. 
Table A.7-9

Sediment PSM Sample Results for Isotopes Detected above Minimum Detectable Concentrations at CAS 12-30-14, Cellar

\begin{tabular}{|c|c|c|c|c|c|}
\hline \multirow{2}{*}{$\begin{array}{l}\text { Sample } \\
\text { Location }\end{array}$} & \multirow{2}{*}{$\begin{array}{l}\text { Sample } \\
\text { Number }\end{array}$} & \multirow{2}{*}{$\begin{array}{c}\text { Thickness } \\
\text { (ft) }\end{array}$} & \multicolumn{3}{|c|}{ Contaminants of Potential Concern (pCi/g) } \\
\hline & & & Uranium-234 & Uranium-235 & Uranium-238 \\
\hline \multicolumn{3}{|c|}{ PSM Criteria ${ }^{a}$} & 143 & 17.6 & 105 \\
\hline E-Sediment & 234E002 & $1.0^{\mathrm{b}}$ & 0.97 & -- & 0.98 \\
\hline E-Sediment & 234E003 & $1.0^{\mathrm{b}}$ & 0.93 & 0.053 & 1 \\
\hline E-Sediment & 234E004 & $1.0^{\mathrm{b}}$ & 0.85 & -- & 1.03 \\
\hline
\end{tabular}

${ }^{a}$ Taken from the construction, commercial, industrial land use scenario in Table 2.1 of the NCRP Report No. 129 , Recommended Screening Limits for Contaminated Surface Soil and Review Factors Relevant to Site-Specific Studies (NCRP, 1999). The values provided in this source document were scaled to a 25-millirem-per-year dose.

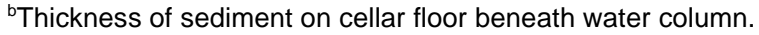

$\mathrm{ft}=$ Foot

NCRP $=$ National Council on Radiation Protection and Measurements

$\mathrm{pCi} / \mathrm{g}=$ Picocuries per gram

$\mathrm{PSM}=$ Potential source material

$--=$ Not detected above minimum detectable concentrations

\section{A.7.4 Revised Conceptual Site Model}

The results of the CAI at CAS 12-30-14 did not contradict the CSM. No revision of the CSM was necessary. 


\section{A.8.0 Waste Management}

Sections A.8.1 through A.8.3 address IDW, and Sections A.8.4 and A.8.5 address potential disposal and remediation of the wastes.

\section{A.8.1 Investigation-Derived Waste}

Investigation-derived waste was generated during the field investigation activities of CAU 234. The waste streams generated include decontamination rinsate water, disposable personal protective equipment (PPE), and disposable sampling equipment. Investigation-derived waste was segregated to the greatest extent possible, and waste minimization techniques were integrated into the field activities to reduce the amount of waste generated. Controls were in place to minimize the use of hazardous materials and the unnecessary generation of hazardous and/or mixed waste.

Decontamination activities were planned and executed to minimize the volume of rinsate generated.

One drum of IDW was generated during the investigation; it contained the decontamination rinsate from the equipment used at CAS 03-09-02.

\section{A.8.2 IDW Waste Streams}

Investigation-derived waste generated during the investigation was segregated into the following waste streams:

- Disposable PPE and sampling equipment

- Decontamination rinsate

- Debris including, but not limited to: plastic sheeting, glass/plastic sample jars, PPE, soil, sampling scoops, aluminum foil, and bowls

Sanitary industrial waste was inspected and disposed of in designated sanitary industrial waste bins located at Building 23-153 and allocated for disposal at the NTS industrial waste landfill. 


\section{A.8.3 Waste Characterization}

The IDW waste streams were characterized as sanitary waste based on process knowledge, site environmental samples, and direct samples of the waste. The characterization and disposition was based on federal and state regulations, permit limitations, and acceptance criteria.

\section{A.8.4 Waste Disposal}

The rinsate waste stream was solidified and shipped to the Area 9 U10c Landfill. The cellar liquids and cellar sediment have been evaluated for potential release considerations and were not PSM.

\section{A.8.5 Potential Remediation Wastes}

Table A.8-1 presents a summary of the estimated volumes, characterizations, and disposition pathways of these potential waste streams for each applicable CAS. 
Table A.8-1

Page A-65 of A-75

CAU 234 Projected Waste Inventory and Preliminary Disposal Recommendation Summary

\begin{tabular}{|c|c|c|c|c|c|c|c|c|}
\hline CAS & Waste Item & Volume Capacity & $\begin{array}{l}\text { Process } \\
\text { Knowledge }\end{array}$ & $\begin{array}{l}\text { Analytical } \\
\text { Suite }\end{array}$ & $\begin{array}{l}\text { Landfill } \\
\text { Limits }\end{array}$ & $\begin{array}{l}\text { NTS } \\
\text { POC }\end{array}$ & $\begin{array}{l}\text { Lagoon } \\
\text { Criteria }\end{array}$ & $\begin{array}{c}\text { Recommended } \\
\text { Disposal Pathway }\end{array}$ \\
\hline 02-09-48 & Rusted drum & $55 \mathrm{gal}$ & $\mathrm{N} / \mathrm{A}$ & Sanitarya, b & Meets & Meets & $\mathrm{N} / \mathrm{A}$ & Area 9 U10c Landfilla \\
\hline \multirow{3}{*}{ 03-09-02 } & $\begin{array}{l}\text { Decontamination } \\
\text { rinsate }\end{array}$ & $5 \mathrm{gal}$ & $\mathrm{N} / \mathrm{A}$ & Sanitary ${ }^{b}$ & $N / A$ & Meets & Meets & Area 23 Lagoon $^{\mathrm{b}}$ \\
\hline & \multirow{2}{*}{$\begin{array}{l}\text { Four pieces of } \\
\text { loose empty } \\
\text { metal; PVC pipe } \\
\text { section }\end{array}$} & $\mathrm{N} / \mathrm{A}$ & $\mathrm{N} / \mathrm{A}$ & $\mathrm{N} / \mathrm{A}$ & Meets & Meets & $\mathrm{N} / \mathrm{A}$ & Area 9 U10c Landfill ${ }^{a}$ \\
\hline & & $\mathrm{N} / \mathrm{A}$ & $\mathrm{N} / \mathrm{A}$ & $\mathrm{N} / \mathrm{A}$ & Meets & Meets & $\mathrm{N} / \mathrm{A}$ & Area 9 U10c Landfill ${ }^{a}$ \\
\hline \multirow[b]{2}{*}{$12-09-01$} & $\begin{array}{l}\text { Metal cylinder, } \\
\text { metal pipe }\end{array}$ & $\begin{array}{c}\text { Cylinder - } 45 \mathrm{ft}^{3} \\
\text { Pipe - } 20 \mathrm{ft} \text { in length }\end{array}$ & $\mathrm{N} / \mathrm{A}$ & Sanitary ${ }^{a, b}$ & Meets & Meets & $\mathrm{N} / \mathrm{A}$ & Area 9 U10c Landfilla \\
\hline & $\begin{array}{l}\text { Glass and metal } \\
\text { trash from inside } \\
\text { the cylinder }\end{array}$ & $\mathrm{N} / \mathrm{A}$ & $\mathrm{N} / \mathrm{A}$ & $\mathrm{N} / \mathrm{A}$ & Meets & Meets & $\mathrm{N} / \mathrm{A}$ & Area 9 U10c Landfilla \\
\hline \multirow{4}{*}{$12-09-08$} & $\begin{array}{l}\text { Crushed drums, } \\
\text { metal pipe }\end{array}$ & $\begin{array}{l}\text { Drums - 30-gal } \\
\text { Pipe - } 4 \mathrm{ft} \text { in length }\end{array}$ & $\mathrm{N} / \mathrm{A}$ & Sanitary ${ }^{a, b}$ & Meets & Meets & $\mathrm{N} / \mathrm{A}$ & Area 9 U10c Landfill \\
\hline & Drum & $55 \mathrm{gal}$ & $\mathrm{N} / \mathrm{A}$ & Sanitary ${ }^{\mathrm{a}, \mathrm{b}}$ & Meets & Meets & N/A & Area 9 U10c Landfill \\
\hline & Rusted drum & Unknown & $\mathrm{N} / \mathrm{A}$ & $\mathrm{N} / \mathrm{A}$ & Meets & Meets & $\mathrm{N} / \mathrm{A}$ & Area 9 U10c Landfill \\
\hline & $\begin{array}{l}\text { Motor vehicle } \\
\text { exhaust pipe }\end{array}$ & Unknown & $\mathrm{N} / \mathrm{A}$ & $\mathrm{N} / \mathrm{A}$ & Meets & Meets & $\mathrm{N} / \mathrm{A}$ & Area 9 U10c Landfill \\
\hline
\end{tabular}

aAnalytical results of surrounding soil was used to characterize the waste items in addition to radiological screening to meet landfill acceptance criteria.

${ }^{b}$ Full analytical suite consists of the following analyses: Total VOCs, Total SVOCs, PCBs, RCRA Metals, TPH-DRO, and radiological (gamma, isotopic uranium, isotopic plutonium, and strontium-90).

$\mathrm{DRO}=$ Diesel-range organics

$\mathrm{ft}=$ Foot

$\mathrm{ft}^{3}=$ Cubic foot

gal $=$ Gallon

$\mathrm{N} / \mathrm{A}=$ Not applicable

$\mathrm{PCB}=$ Polychlorinated biphenyl
$\mathrm{POC}=$ Performance objective criteria

PVC = Polyvinyl chloride

$\mathrm{RCRA}=$ Resource Conservation and Recovery Act

SVOC = Semivolatile organic compound

$\mathrm{TPH}=$ Total petroleum hydrocarbons

VOC $=$ Volatile organic compound 


\section{A.9.0 Quality Assurance}

This section contains a summary of QA/QC measures implemented during the sampling and analysis activities conducted in support of the CAU $234 \mathrm{CAI}$. The following sections discuss the data validation process, QC samples, and nonconformances. A detailed evaluation of the DQIs is presented in Appendix B.

Laboratory analyses were conducted for samples used in the decision-making process to provide a quantitative measurement of any COPCs present. Rigorous QA/QC was implemented for all laboratory samples including documentation, verification and validation of analytical results, and affirmation of DQI requirements related to laboratory analysis. Detailed information regarding the QA program is contained in the Industrial Sites QAPP (NNSA/NV, 2002).

\section{A.9.1 Data Validation}

Data validation was performed in accordance with the Industrial Sites QAPP and approved protocols and procedures. All laboratory data from samples collected and analyzed for CAU 234 were evaluated for data quality in a tiered process and are presented in Sections A.9.1.1 through A.9.1.3. Data were reviewed to ensure that samples were appropriately processed and analyzed, and the results were evaluated using validation criteria. Documentation of the data qualifications resulting from these reviews is retained in project files as a hard copy and electronic media.

One hundred percent of the data analyzed as part of this investigation were subjected to Tier I and Tier II evaluations. A Tier III evaluation was performed on 10 percent of the data analyzed.

\section{A.9.1.1 Tier I Evaluation}

Tier I evaluation for chemical and radiochemical analysis examines, but is not limited to:

- Sample count/type consistent with chain of custody.

- Analysis count/type consistent with chain of custody.

- Correct sample matrix.

- Significant problems and/or nonconformances stated in cover letter or case narrative.

- Completeness of certificates of analysis.

- Completeness of Contract Laboratory Program (CLP) or CLP-like packages. 
- Completeness of signatures, dates, and times on chain of custody.

- Condition-upon-receipt variance form included.

- Requested analyses performed on all samples.

- Date received/analyzed given for each sample.

- Correct concentration units indicated.

- Electronic data transfer supplied.

- Results reported for field and laboratory QC samples.

- Whether or not the deliverable met the overall objectives of the project.

\section{A.9.1.2 Tier II Evaluation}

Tier II evaluation for chemical analysis examines, but is not limited to:

- Correct detection limits achieved.

- Sample date, preparation date, and analysis date for each sample.

- Holding time criteria met.

- Quality control batch association for each sample.

- Cooler temperature upon receipt.

- Sample pH for aqueous samples, as required.

- Detection limits properly adjusted for dilution, as required.

- Blank contamination evaluated and applied to sample results/qualifiers.

- Matrix spike/MSD percent recoveries (\%R) and relative percent differences (RPDs) evaluated and qualifiers applied to laboratory results, as necessary.

- Field duplicate RPDs evaluated using professional judgment and qualifiers applied to laboratory results, as necessary.

- Laboratory duplicate RPDs evaluated and qualifiers applied to laboratory results, as necessary.

- Surrogate $\% \mathrm{R}$ evaluated and qualifiers applied to laboratory results, as necessary.

- Laboratory control sample (LCS) \%R evaluated and qualifiers applied to laboratory results, as necessary.

- Initial and continuing calibration evaluated and qualifiers applied to laboratory results, as necessary.

- Internal standard evaluation.

- Mass spectrometer tuning criteria.

- Organic compound quantitation. 
- Inductively coupled plasma interference check sample evaluation.

- Graphite furnace atomic absorption QC.

- Inductively coupled plasma serial dilution effects.

- Recalculation of 10 percent of laboratory results from raw data.

Tier II evaluation for radiochemical analysis examines, but is not limited to:

- Correct detection limits achieved.

- Blank contamination evaluated and, if significant, qualifiers are applied to sample results.

- Certificate of Analysis consistent with data package documentation.

- Quality control sample results (duplicates, LCSs, laboratory blanks) evaluated and used to determine laboratory result qualifiers.

- Sample results, uncertainty, and MDC evaluated.

- Detector system calibrated with National Institute of Standards and Technology (NIST)traceable sources.

- Calibration sources preparation was documented, demonstrating proper preparation and appropriateness for sample matrix, emission energies, and concentrations.

- Detector system response to daily or weekly background and calibration checks for peak energy, peak centroid, peak full-width half-maximum, and peak efficiency, depending on the detection system.

- Tracers NIST-traceable, appropriate for the analysis performed, and recoveries that met QC requirements.

- Documentation of all QC sample preparation complete and properly performed.

- Spectra lines, photon emissions, particle energies, peak areas, and background peak areas support the identified radionuclide and its concentration.

\section{A.9.1.3 Tier III Evaluation}

The Tier III review is an independent examination of the Tier II evaluation. A Tier III review of 10 percent of the sample analytical data was performed by TLI Solutions, of Golden, Colorado. 
Tier II and Tier III results were compared and where differences were noted, data were reviewed and did not result in any changes to the data. This review included the following additional evaluations:

Review of:

- Case narrative, chain of custody, and sample receipt forms

- Lab qualifiers (applied appropriately)

- Method of analyses performed as dictated by the chain of custody

- Raw data, including chromatograms, instrument printouts, preparation logs, and analytical $\operatorname{logs}$

- Manual integrations to determine whether the instrument response is appropriate

- Data package for completeness

Determine sample results qualifiers through the evaluation of (but not limited to):

- Tracers and QC sample results (e.g., duplicates, LCSs, blanks, MSs) evaluated and used to determine sample results qualifiers

- Sample preservation, sample preparation/extraction and run logs, sample storage, and holding time

- Instrument and detector tuning

- Initial and continuing calibrations

- Calibration verification (initial, continuing, second source)

- Retention times

- Second column and/or second detector confirmation

- Mass spectra interpretation

- Interference check samples and serial dilutions

- Post-digestion spikes and method of standard additions

- Breakdown evaluations 
Calculation checks of:

- At least one analyte per QC sample checked for its recovery

- At least one analyte per initial calibration curve, continuing calibration verification, and second source recovery

- At least one analyte per sample that contains positive results (hits). Radiochemical results only require calculation checks on activity concentrations (not error)

There is also verification that the target compound detects identified in the raw data are reported on the results form. There is also a document of any anomalies found during the review for the laboratory to clarify or rectify. The contractor is notified of any anomalies found.

\section{A.9.2 Field Quality Control Samples}

Field QC samples consisted of nine trip blanks, one equipment rinsate blank, four field blanks, one source blank, five MS/MSDs, and five FDs collected and submitted for analysis by the laboratory analytical methods shown in Table A.2-2. The QC samples were assigned individual sample numbers and sent to the laboratory "blind." Additional samples were selected by the laboratory to be analyzed as laboratory duplicates.

During the CAI, five FDs were sent as blind samples to the laboratory to be analyzed for the investigation parameters listed in Table A.2-2. For these samples, the duplicate results precision (i.e., RPDs between the environmental sample results and their corresponding FD sample results) were evaluated. All duplicate precision targets were met.

\section{A.9.2.1 Laboratory Quality Control Samples}

Analyses of preparation (PB) blanks were performed on each sample delivery group (SDG) for inorganics. Analysis for surrogate spikes and method blanks (MBs) were performed on each SDG for organics only. Initial and continuing calibration and LCSs were performed for each SDG. The results of these analyses were used to qualify associated environmental sample results. Documentation of data qualifications resulting from the application of these guidelines is retained in project files as both hard copy and electronic media. 
The laboratory included a PB, LCS, and laboratory duplicate sample with each batch of field samples analyzed for radionuclides.

\section{A.9.3 Field Nonconformances}

There were no field nonconformances identified for the CAI.

\section{A.9.4 Laboratory Nonconformances}

Laboratory nonconformances are generally due to inconsistencies in the analytical instrumentation operation, sample preparations, extractions, missed holding times, and fluctuations in internal standard and calibration results. Five nonconformances were issued by the laboratory. These laboratory nonconformances were accounted for and resolved during the data validation and qualification process. 


\section{A.10.0 Summary}

Organic, inorganics, and radionuclide contaminants detected in environmental samples during the CAI were evaluated against FALs to determine the nature and extent of COCs for CAU 234.

Assessment of the data generated from investigation activities indicates no FALs were exceeded for any of the COPCs within the CASs of CAU 234. 


\section{A.11.0 References}

BN, see Bechtel Nevada.

Bechtel Nevada. 1995. Nevada Test Site Performance Objective for Certification of Nonradioactive Hazardous Waste, Rev. 0, G-E11/96.01. Las Vegas, NV.

Bechtel Nevada. 1999. An Aerial Radiological Survey of the Nevada Test Site. Nevada Test Site, Nevada, DOE/NV/11718-324. December. Las Vegas, NV.

CFR, see Code of Federal Regulations.

Code of Federal Regulations. 2006. Title 40 CFR Part 261, "Identification and Listing of Hazardous Waste." Washington, DC: U.S. Government Printing Office.

DOE, see U.S. Department of Energy.

EMSL/ORD, see Environmental Monitoring and Support Laboratory Office of Research and Development.

EPA, see U.S. Environmental Protection Agency.

Environmental Monitoring and Support Laboratory Office of Research and Development. 1980. Prescribed Procedures for Measurement of Radioactivity in Drinking Water, EPA-600/4-80-032. August. Cincinnati, OH: U.S. Environmental Protection Agency.

Moore, J., Science Applications International Corporation. 1999. Memorandum to M. Todd (SAIC), "Background Concentrations for NTS and TTR Soil Samples," 3 February. Las Vegas, NV.

NAC, see Nevada Administrative Code.

NBMG, see Nevada Bureau of Mines and Geology.

NCRP, see National Council on Radiation Protection and Measurements.

NNSA/NSO, see U.S. Department of Energy, National Nuclear Security Administration Nevada Site Office.

NNSA/NV, see U.S. Department of Energy, National Nuclear Security Administration Nevada Operations Office. 
National Council on Radiation Protection and Measurements. 1999. Recommended Screening Limits for Contaminated Surface Soil and Review of Factors Relevant to Site-Specific Studies, Report No. 129. Bethesda, MD.

Nevada Administrative Code. 2006. NAC 445A.2272, "Contamination of Soil: Establishment of Action Levels." Carson City, NV. As accessed at http://www.leg.state.nv.us/nac on 29 January 2008.

Nevada Bureau of Mines and Geology. 1998. Mineral and Energy Resource Assessment of the Nellis Air Force Range, Open-File Report 98-1. Reno, NV.

RSL, see Remote Sensing Laboratory. Las Vegas, NV.

Remote Sensing Laboratory. 1989. Aerial photograph “6612-146.” Las Vegas, NV.

Remote Sensing Laboratory. 1993. Aerial photograph "7427-04\#2” showing Area 2 mud and crater storage sumps, 1 June. Las Vegas, NV: EG\&G Energy Measurements, Inc.

Remote Sensing Laboratory. 1999. Digital Orthophoto Quarter Quads. Las Vegas, NV.

SNJV, see Stoller-Navarro Joint Venture.

SNJV GIS, see Stoller-Navarro Joint Venture Geographic Information Systems.

Stoller-Navarro Joint Venture. 2006. Model Statement of Work for Analytical Laboratories. Las Vegas, NV.

Stoller-Navarro Joint Venture Geographic Information Systems. 2008. ESRI ArcGIS Software.

U.S. Department of Energy. 1993. Radiation Protection of the Public and the Environment, DOE Order 5400.5, Change 2. Washington, DC.

U.S. Department of Energy. 1997. The Procedures Manual of the Environmental Measurements Laboratory, HASL-300. Vol. 1 Ed. 28, HASL-300. February. New York, NY.

U.S. Department of Energy, National Nuclear Security Administration Nevada Operations Office. 2002. Industrial Sites Quality Assurance Project Plan, Nevada Test Site, Nevada, Rev. 3, DOE/NV--372. Las Vegas, NV.

U.S. Department of Energy, National Nuclear Security Administration Nevada Site Office. 2006a. Closure Report for Corrective Action Units 530, 531, 532, 533, 534, 535: NTS Mud Pits, Nevada Test Site, Nevada, Rev. 0, DOE/NV--1131. Las Vegas, NV. 
U.S. Department of Energy, National Nuclear Security Administration Nevada Site Office. 2006b. Industrial Sites Project Establishment of Final Action Levels, Rev. 0, DOE/NV--1107. Las Vegas, NV.

U.S. Department of Energy, National Nuclear Security Administration Nevada Site Office. 2007. Corrective Action Investigation Plan for Corrective Action Unit 234: Mud Pits, Cellars, and Mud Spills, Nevada Test Site, Nevada, Rev. 0, DOE/NV--1216. Las Vegas, NV.

U.S. Environmental Protection Agency. 1996. Test Methods for Evaluating Solid Waste, Physical/Chemical Methods, SW-846, 3rd Edition, CD-ROM PB97-501928GEI. Washington, DC.

U.S. Environmental Protection Agency. 2004 (as revised). Region 9 Preliminary Remediation Goals (PRGs). As accessed at www.epa.gov/region09/waste/sfund/prg/htm on 16 January 2008. 
Appendix B

\section{Data Assessment}




\section{B.1.0 Data Assessment}

The DQA process is the scientific evaluation of the actual investigation results to determine whether the DQO criteria established in the CAU 234 CAIP (NNSA/NSO, 2007) were met and whether DQO decisions can be resolved at the desired level of confidence. The DQO process ensures that the right type, quality, and quantity of data will be available to support the resolution of those decisions at an appropriate level of confidence. Using both the DQO and DQA processes helps to ensure that DQO decisions are sound and defensible.

The DQA involves five steps that begin with a review of the DQOs and end with an answer to the DQO decisions. The five steps are briefly summarized as follows:

Step 1: Review DQOs and Sampling Design - Review the DQO process to provide context for analyzing the data. State the primary statistical hypotheses; confirm the limits on decision errors for committing false negative (Type I) or false positive (Type II) decision errors; and review any special features, potential problems, or deviations to the sampling design.

Step 2: Conduct a Preliminary Data Review - Perform a preliminary data review by reviewing QA reports and inspecting the data both numerically and graphically, validating and verifying the data to ensure that the measurement systems performed in accordance with the criteria specified, and using the validated dataset to determine whether the quality of the data is satisfactory.

Step 3: Select the Test - Select the test based on the population of interest, population parameter, and hypotheses. Identify the key underlying assumptions that could cause a change in one of the DQO decisions.

Step 4: Verify the Assumptions - Perform tests of assumptions. If data are missing or are censored, determine the impact on DQO decision error.

Step 5: Draw Conclusions from the Data - Perform the calculations required for the test. 


\section{B.1.1 Review DQOs and Sampling Design}

This section contains a review of the DQO process presented in Appendix A of the CAU 234 CAIP (NNSA/NSO, 2007). The DQO decisions are presented with the DQO provisions to limit false negative or false positive decision errors. Special features, potential problems, or any deviations to the sampling design are also presented.

\section{B.1.1.1 Decision I}

The Decision I statement as presented in the CAU 234 CAIP is: "Is any COC present within the CAS?" (NNSA/NSO, 2007).

\section{Decision I Rules:}

- If the population parameter of any COPC in a target population exceeds the FAL for that COPC, then that COPC is identified as a COC.

- If a COC is detected, then the Decision II statement must be resolved.

- If COCs are not identified, then the investigation is complete.

\section{B.1.1.1.1 DQO Provisions To Limit False Negative Decision Error}

A false negative decision error (where consequences are more severe) was controlled by meeting the following criteria:

1. Having a high degree of confidence that locations selected will identify COCs if present anywhere within the CAS.

2. Having a high degree of confidence that analyses conducted will be sufficient to detect any COCs present in the samples at an acceptable level of sensitivity.

3. Having a high degree of confidence that the dataset is of sufficient quality and completeness.

\section{Criterion 1:}

The following methods (stipulated in the CAU 234 DQOs [NNSA/NSO, 2007]) were used in selecting sample locations. 
1. Selection of sampling locations associated with surface and subsurface staining, odors, presence of debris, and other items was accomplished by visual field observations.

2. Selection of sampling locations associated with professional judgment based on acceptable knowledge was accomplished by:

- Source and location of release

- Chemical nature and fate properties

- Physical transport pathways and properties

- Transport drivers

\section{Criterion 2:}

All samples were analyzed using the analytical methods listed in Table 3-3 of the CAIP and for the chemical and radiological constituents listed in Section 3.2 of the CAIP (NNSA/NSO, 2007).

Table B.1-1 provides a reconciliation of samples analyzed to the planned analytical program.

Table B.1-1

CAU 234 Analyses Performed

\begin{tabular}{|c|c|c|c|c|c|c|c|c|c|}
\hline CAS & $\begin{array}{l}\text { O } \\
\stackrel{0}{0} \\
\frac{\pi}{0} \\
\stackrel{0}{0}\end{array}$ & 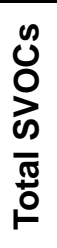 & $\begin{array}{l}\mathscr{n} \\
\text { U్ }\end{array}$ & $\frac{\frac{00}{\pi}}{\frac{\mathbb{d}}{2}}$ & 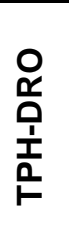 & 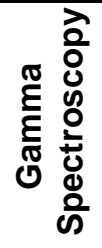 & 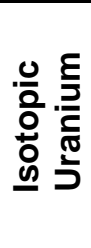 & 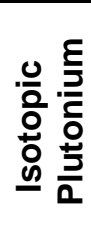 & 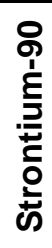 \\
\hline $02-09-48$ & $\mathrm{RS}$ & $\mathrm{RS}$ & $\mathrm{RS}$ & $\mathrm{RS}$ & $\mathrm{RS}$ & $\mathrm{RS}$ & $\mathrm{RS}$ & $\mathrm{RS}$ & $\overline{R S}$ \\
\hline 03-09-02 & $\mathrm{RS}$ & $\mathrm{RS}$ & $\mathrm{RS}$ & $\mathrm{RS}$ & $\mathrm{RS}$ & $\mathrm{RS}$ & $\mathrm{RS}$ & $\mathrm{RS}$ & $\mathrm{RS}$ \\
\hline $12-09-01$ & $\mathrm{RS}$ & $\mathrm{RS}$ & $\mathrm{RS}$ & RS & $\mathrm{RS}$ & $\mathrm{RS}$ & $\mathrm{RS}$ & $\mathrm{RS}$ & $\mathrm{RS}$ \\
\hline $12-09-08$ & $\mathrm{RS}$ & $\mathrm{RS}$ & $\mathrm{RS}$ & $\mathrm{RS}$ & $\mathrm{RS}$ & $\mathrm{RS}$ & $\mathrm{RS}$ & $\mathrm{RS}$ & $\mathrm{RS}$ \\
\hline $12-30-14$ & RS & $\mathrm{RS}$ & RS & RS & $\mathrm{RS}$ & RS & RS & $\mathrm{RS}$ & $\mathrm{RS}$ \\
\hline
\end{tabular}

$\mathrm{DRO}=$ Diesel-range organics

RS $=$ Required and submitted

$\mathrm{PCB}=$ Polychlorinated biphenyl

SVOC $=$ Semivolatile organic compound

$\mathrm{TPH}=$ Total petroleum hydrocarbons

$\mathrm{VOC}=$ Volatile organic compound

Samples were submitted for all of the analytical methods specified in the analytical program specified in Section 3.2 of the CAIP (NNSA/NSO, 2007).

Sample results were assessed against the acceptance criterion for the DQI of sensitivity as defined in the Industrial Sites QAPP (NNSA/NV, 2002). The sensitivity acceptance criterion defined in the 
CAIP is that analytical detection limits will be less than the corresponding action level

(NNSA/NSO, 2007). This criterion was achieved for the analytical results for CAU 234.

\section{Criterion 3:}

To satisfy the third criterion, the entire dataset, as well as individual sample results, were assessed against the acceptance criteria for the DQIs of precision, accuracy, comparability, completeness, and representativeness, as defined in the Industrial Sites QAPP (NNSA/NV, 2002). The DQI acceptance criteria are presented in Table 6-1 of the CAIP (NNSA/NSO, 2007).

\section{$\underline{\text { Precision }}$}

Precision was evaluated as described in Section 6.2 of the CAIP (NNSA/NSO, 2007). While three samples were qualified for lead and one for $\mathrm{Pu}-239 / 240$ duplicate precision for the laboratory QC sample, the 80 percent acceptance criteria was met (Table B.1-2).

Table B.1-2

Precision Qualifications for CAU 234

\begin{tabular}{|c|c|c|c|c|c|}
\hline Constituent & CAS Number & Analysis & $\begin{array}{c}\text { Samples } \\
\text { Qualified }\end{array}$ & $\begin{array}{c}\text { Total } \\
\text { Measurements }\end{array}$ & $\begin{array}{c}\text { Percent } \\
\text { Acceptable }\end{array}$ \\
\hline \hline Lead & $7439-92-1$ & METALS & 3 & 32 & 90.6 \\
\hline Plutonium-239/240 & $15117-48-3$ & PLUTONIUM & 1 & 32 & 96.9 \\
\hline
\end{tabular}

\section{Sensitivity}

Sensitivity was evaluated as described in Section 6.2 of the CAIP (NNSA/NSO, 2007). The dataset is acceptable for the DQI sensitivity.

\section{Accuracy}

Accuracy was evaluated as described in Section 6.2 of the CAIP (NNSA/NSO, 2007). Table B.1-3 provides the chemical accuracy analysis results for all constituents qualified for accuracy. Accuracy rates are above the CAIP criterion of 80 percent. There were no radiological data qualified for accuracy. 
Table B.1-3

Accuracy Measurements for CAU 234

\begin{tabular}{|c|c|c|c|c|c||}
\hline Constituent & $\begin{array}{c}\text { CAS } \\
\text { Number }\end{array}$ & $\begin{array}{c}\text { User Test } \\
\text { Panel }\end{array}$ & $\begin{array}{c}\text { Number of } \\
\text { Measurements } \\
\text { Qualified }\end{array}$ & $\begin{array}{c}\text { Number of } \\
\text { Measurements } \\
\text { Performed }\end{array}$ & $\begin{array}{c}\text { Percent } \\
\text { within } \\
\text { Criteria }\end{array}$ \\
\hline \hline Benzene & $71-43-2$ & EPA 8260C & 1 & 32 & 96.9 \\
\hline Toluene & $108-88-3$ & EPA 8260C & 1 & 32 & 96.9 \\
\hline Chlorobenzene & $108-90-7$ & EPA 8260C & 2 & 32 & 93.8 \\
\hline Lead & $7439-92-1$ & EPA 6010B & 3 & 32 & 90.6 \\
\hline
\end{tabular}

CAS $=$ Chemical Abstract Service

EPA $=$ Environmental Protection Agency, SW-846 methods (EPA, 1999 and 2002)

\section{$\underline{\text { Representativeness }}$}

The DQO process as identified in Appendix A of the CAU 234 CAIP (NNSA/NSO, 2007) was used to address sampling and analytical requirements for CAU 234. During this process, appropriate locations were selected that enabled the samples collected to be representative of the population parameters identified in the DQO (the most likely locations to contain contamination and locations that bound COCs). The sampling locations identified in the Criterion 1 discussion meet this criterion. Therefore, the analytical data acquired during the CAU $234 \mathrm{CAI}$ are considered representative of the population parameters.

\section{Comparability}

Field sampling, as described in the CAU 234 CAIP (NNSA/NSO, 2007), was performed and documented in accordance with approved procedures that are comparable to standard industry practices. Approved analytical methods and procedures per DOE were used to analyze, report, and validate the data. These are comparable to other methods used not only in industry and government practices, but most importantly are comparable to other investigations conducted for the NTS. Therefore, project datasets are considered comparable to other datasets generated using these same standardized DOE procedures, thereby meeting DQO requirements.

Also, standard, approved field and analytical methods ensured that data were appropriate for comparison to the investigation action levels specified in the CAIP. 


\section{Completeness}

The CAU 234 CAIP (NNSA/NSO, 2007) defines acceptable criteria for completeness to be that the dataset is sufficiently complete to be able to make the DQO decisions. This is initially evaluated as 80 percent of CAS-specific non-critical analytes identified in the CAIP having valid results and 100 percent of critical analytes (including Decision II samples) having valid results. No critical analytes were identified for CAU 234.

Rejected data (either qualified as rejected or data that failed the criterion of sensitivity) are not used in the resolution of DQO decisions and are not counted toward meeting the completeness acceptance criterion. However, no data were rejected in the analyses for CAU 234. All data are within acceptable criteria.

\section{B.1.1.2 DQO Provisions To Limit False Positive Decision Error}

The false positive decision error was controlled by assessing the potential for false positive analytical results. Quality assurance/QC samples such as field blanks, trip blanks, LCSs, and MBs were used to determine whether a false positive analytical result may have occurred. This provision is evaluated during the validation process where appropriate qualifications are applied.

Proper decontamination of sampling equipment and the use of certified clean sampling equipment and containers also minimized the potential for cross contamination that could lead to a false positive analytical result.

\section{B.1.1.2 Decision II}

Decision II as presented in the CAU 234 CAIP is: "If a COC is present, is sufficient information available to evaluate appropriate corrective action alternatives?" (NNSA/NSO, 2007). Sufficient information is defined to include:

- Identifying the volume of media containing any COC bounded by analytical sample results in lateral and vertical directions.

- The information needed to determine potential remedial waste types. 
Sample results for samples collected at the CASs of CAU 234 confirmed the absence of COCs. Therefore, no remediation is necessary, and no alternatives need be considered.

\section{B.1.1.3 Sampling Design}

The CAIP (NNSA/NSO, 2007) made the following commitments for sampling:

All samples collected were based on judgmental design. All biased locations will have soil samples collected beneath and/or adjacent to the items of interest to identify releases of contaminants and investigate the integrity of any formally enclosed items (e.g., drums, pipes). For CAS 03-09-02, samples were collected from the true low points within each mud pit feature as being representative of the location of the potential highest concentration of contaminants.

Result: All samples were collected at each CAS by hand excavation and soil samples were collected adjacent to and from beneath the required components such as the base of drums, pipes, and cylindrical debris. Corrective Action Site 12-30-14 samples of liquid and sediment were collected to evaluate PSM.

\section{B.1.2 Conduct a Preliminary Data Review}

A preliminary data review was conducted by reviewing QA reports and inspecting the data. The contract analytical laboratories generate a QA non-conformance report when data quality does not meet contractual requirements. All data received from the analytical laboratories met contractual requirements, and a QA non-conformance report was not generated. Data were validated and verified to ensure that the measurement systems performed in accordance with the criteria specified. The validated dataset quality was found to be satisfactory.

\section{B.1.3 Select the Test and Identify Key Assumptions}

The test for making DQO Decision I was the comparison of the maximum analyte result from each CAS to the corresponding FAL. The test for making DQO Decision II was the comparison of all COC analyte results from each bounding sample to the corresponding FALs.

The key assumptions that could impact a DQO decision are listed in Table B.1-4. 
Table B.1-4

\section{Key Assumptions}

\begin{tabular}{|c|c|}
\hline Exposure Scenario & $\begin{array}{l}\text { Site workers are only exposed to contaminants of concern (COCs) through oral } \\
\text { ingestion, inhalation, external exposure to radiation, or dermal contact (by absorption) } \\
\text { of COCs absorbed onto the soils. } \\
\text { Exposure to contamination is limited to industrial site workers, } \\
\text { construction/remediation workers, and military personnel conducting training. }\end{array}$ \\
\hline Affected Media & $\begin{array}{l}\text { Surface soil, shallow subsurface soil, and potentially perched (shallow) groundwater. } \\
\text { Deep groundwater contamination is not a concern. } \\
\text { Contaminants migrating to regional aquifers are not considered. }\end{array}$ \\
\hline $\begin{array}{l}\text { Location of } \\
\text { Contamination/Release Points }\end{array}$ & $\begin{array}{l}\text { The area of contamination is contiguous. } \\
\text { The extent of COC concentration decreases away from the area of contamination. }\end{array}$ \\
\hline Transport Mechanisms & $\begin{array}{l}\text { Surface transport may occur as a result of a spill or stormwater runoff. } \\
\text { Surface transport beyond shallow substrate is not a concern. }\end{array}$ \\
\hline Preferential Pathways & None. \\
\hline $\begin{array}{l}\text { Lateral and Vertical Extent } \\
\text { of Contamination }\end{array}$ & $\begin{array}{l}\begin{array}{l}\text { Subsurface contamination, if present, is contiguous and decreases with distance and } \\
\text { depth from the source. }\end{array} \\
\text { Surface contamination may occur laterally as a result of a spill or stormwater runoff. }\end{array}$ \\
\hline Groundwater Impacts & None. \\
\hline Future Land Use & Nonresidential. \\
\hline $\begin{array}{l}\text { Other Data Quality Objective } \\
\text { Assumptions }\end{array}$ & None. \\
\hline
\end{tabular}

\section{B.1.4 Verify the Assumptions}

The results of the investigation support the key assumptions identified in the CAU 234 DQOs and Table B.1-4.

All data collected during the CAI did not invalidate the CSMs presented in the CAIP (NNSA/NSO, 2007), nor did they necessitate revisions to the CSMs.

\section{B.1.4.1 Other DQO Commitments}

The CAIP (NNSA/NSO, 2007) made the following commitments for sampling:

1. Decision II sampling will consist of defining the extent of contamination where COCs have been confirmed at the Decision I locations. If COCs extend beyond Decision I locations, then additional Decision II samples will be collected from sample locations in the direction outward and potentially in the inferred downgradient direction should the contamination be subsurface. The Decision II samples will be located at an adequate distance from the original sample location and be advanced to provide samples and to profile COC concentrations through the upper and 
lower boundaries of detectable contamination. A clean sample (i.e., COCs are less than PALs) collected from the Decision I and II sampling will define the vertical extent of contamination at the respective locations. A minimum of one analytical result less than the PAL from the vertical direction will be required to define the depth of COC contamination, and the lateral extent of contamination may be defined by sample analysis or based on modeling. The contamination boundaries may need to be extrapolated to give an overall view of the lateral and vertical extent of COC concentrations at the site.

Result: No decision II samples were required to be collected at any CAS at CAU 234, as no COCs are present.

\section{B.1.5 Draw Conclusions from the Data}

This section resolves the two DQO decisions for each of the CAU 234 CASs.

\section{B.1.5.1 Decision Rules for Decision I}

Decision Rule: If the concentration of any COPC in a target population exceeds the FAL for that COPC during the initial investigation, then that COPC is identified as a COC and Decision II sampling will be conducted.

Result: No COCs were identified in any sample from CAU 234.

Decision Rule: If all COPC concentrations are less than the corresponding FALs, then the decision will be no further action.

Result: No COCs were identified in samples collected from all CASs in CAU 234. No further action was identified as the corrective action for these CASs.

\section{B.1.5.2 Decision Rules for Decision II}

Decision Rule: If the observed concentration of any COC in a Decision II sample exceeds the FALs, then additional samples will be collected to complete the determination of the extent.

Result: Samples to define extent were not necessary as no COPCs were detected above their respective FALs during Decision I sampling. 
CAU $234 \mathrm{CADD} / \mathrm{CR}$

Appendix B

Revision: 0

Date: May 2008

Page B-10 of B-11

Decision Rule: If all observed COC population parameters are less than the FALs, then the decision will be that the extent of contamination has been defined in the lateral and/or vertical direction.

Result: No Decision II samples were collected from any CAS at CAU 234. 


\section{B.2.0 References}

EPA, see U.S. Environmental Protection Agency.

NNSA/NSO, see U.S. Department of Energy, National Nuclear Security Administration Nevada Site Office.

NNSA/NV, see U.S. Department of Energy, National Nuclear Security Administration Nevada Operations Office.

U.S. Department of Energy, National Nuclear Security Administration Nevada Operations Office. 2002. Industrial Sites Quality Assurance Project Plan, Nevada Test Site, Nevada, Rev. 3, DOE/NV--372. Las Vegas, NV.

U.S. Department of Energy, National Nuclear Security Administration Nevada Site Office. 2007. Corrective Action Investigation Plan for Corrective Action Unit 234: Mud Pits, Cellars, and Mud Spills, Nevada Test Site, Nevada, Rev. 0, DOE/NV--1216. Las Vegas, NV.

U.S. Environmental Protection Agency. 1999. Contract Laboratory Program National Functional Guidelines for Organic Data Review, EPA 540/R-99/008.

U.S. Environmental Protection Agency. 2002. Contract Laboratory Program National Functional Guidelines for Inorganic Data Review, EPA 540/R-01/-008. 


\section{Appendix C}

\section{Risk Assessment}

UNCONTROLLED when Printed 


\section{C.1.0 Risk Assessment}

The RBCA process used to establish FALs is described in the Industrial Sites Project Establishment of Final Action Levels (NNSA/NSO, 2006). This process conforms with NAC Section 445A.227, which lists the requirements for sites with soil contamination (NAC, 2006a). For the evaluation of corrective actions, NAC Section 445A.22705 (NAC, 2006b) requires the use of ASTM Method E 1739-95 (ASTM, 1995) to "conduct an evaluation of the site, based on the risk it poses to public health and the environment, to determine the necessary remediation standards (i.e., FALs) or to establish that corrective action is not necessary."

The evaluation of the need for corrective action will include the potential for wastes that are present at a site to cause the future contamination of site environmental media if the wastes were to be released. To evaluate the potential for cellar contents (liquid and sediment) to result in the introduction of a $\mathrm{COC}$ to the surrounding environmental media, the following conservative assumptions were made:

- The cellar containment would fail at some point and the contents would be released to the surrounding media.

- The resulting concentration of contaminants in the surrounding media would be equal to the concentration of contaminants in the cellar waste.

- Any liquid contaminant in the cellar exceeding the RCRA TC concentration can result in a COC's introduction to the surrounding media.

Sediment containing a contaminant exceeding an equivalent FAL concentration would be considered to be PSM and would require a corrective action. Cellar liquids with contaminant concentrations exceeding an equivalent TC action level would be considered to be PSM and would require a corrective action.

This section contains documentation of the RBCA process used to establish FALs described in the Industrial Sites Project Establishment of Final Action Levels (NNSA/NSO, 2006). This process defines three tiers (or levels) to establish FALs used to evaluate DQO decisions:

- $\quad$ Tier I - Sample results from source areas (highest concentrations) compared to RBSLs (i.e., PALs) based on generic (non-site-specific) conditions. 
- Tier II - Sample results from exposure points compared to SSTLs calculated using site-specific inputs and Tier I formulas.

- Tier III - Sample results from exposure points compared to SSTLs and points of compliance calculated using chemical fate/transport and probabilistic modeling.

The risk-based corrective action decision process stipulated in the Industrial Sites Project Establishment of Final Action Levels (NNSA/NSO, 2006) is summarized in Figure C.1-1.

\section{C.1.1 A. Scenario}

Corrective Action Unit 234, Mud Pits, Cellars, and Mud Spills, consists of the following 12 inactive sites within Areas 2, 3, 4, 12, and 15 of the NTS:

- 02-09-48, Area 2 Mud Plant \#1

- 02-09-49, Area 2 Mud Plant \#2

- 02-99-05, Mud Spill

- 03-09-02, Mud Dump Trenches

- 04-44-02, Mud Spill

- 04-99-02, Mud Spill

- 12-09-01, Mud Pit

- 12-09-04, Mud Pit

- 12-09-08, Mud Pit

- 12-30-14, Cellar

- 12-99-07, Mud Dump

- 15-09-01, Mud Pit

All of the CASs listed above are inactive and abandoned. Only five of the CASs (02-09-48, 03-09-02, 12-09-01, 12-09-08, and 12-30-14) were sampled during the CAI for the reasons described in the CAU 234 CAIP (NNSA/NSO, 2007).

Corrective Action Site 02-09-48 is a drilling mud sump adjacent to Mud Plant \#2, and contains drilling mud and a rusted 55-gal drum.

Corrective Action Site 03-09-02 is a set of suction and return mud pits, and is divided into a "northern" and "southern" footprint, divided by a service road that runs between them. The drilling mud at this CAS potentially contained radioactivity from an intercepted underground plume of radioactivity from the Sandreef test, conducted several years before the drilling of the U-3kz emplacement hole. 


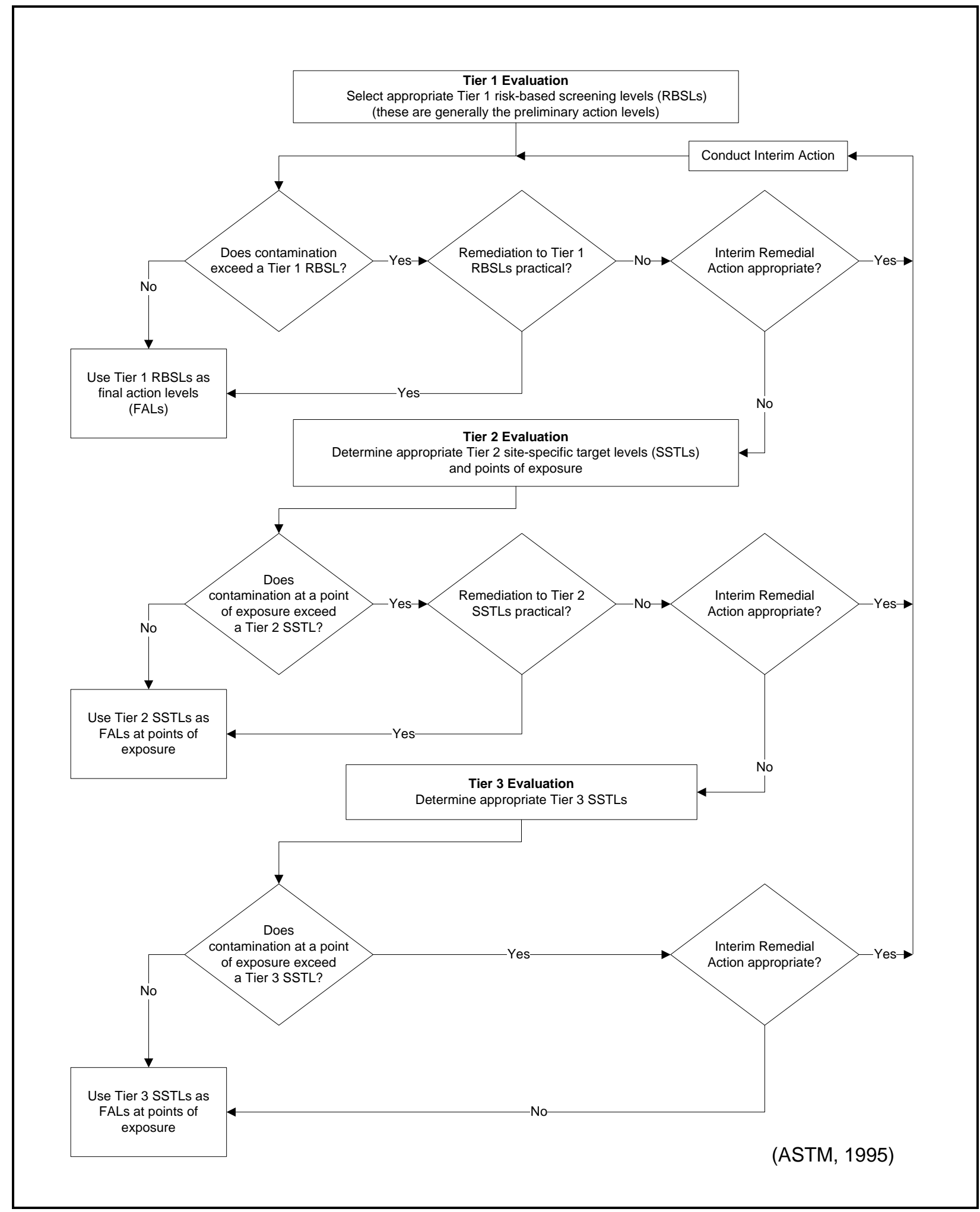

Figure C.1-1

Risk-Based Corrective Action Decision Process 
Corrective Active Site 12-09-01 consists of a mud pit and a piece of unconnected loose metal piping lying on the ground surface and a large cylindrical piece of metal debris approximately $4 \mathrm{ft}$ in diameter containing a large-pore grating on one end. It also contains a hole where an access door would have been on one side of the cylinder. These features were located in the same CAS as the mud pit for CAS 12-09-01, but in separate footprints.

Corrective Active Site 12-09-08 consists of a mud pit containing a piece of metal piping protruding from the top of one of the berm walls, and a set of crushed and rusted 55-gal drums lying on the inside of one of the walls of the berm.

Corrective Active Site 12-30-14 is a post-test cellar that is lined from top to bottom with corrugated steel and measures approximately $10 \mathrm{ft}$ in diameter and is approximately $9 \mathrm{ft}$ deep. Liquid is present within the open cellar and varies in depth according to the amount of rainfall/snowmelt that drains from the surrounding sloped area into the cellar.

\section{C.1.2 B. Site Assessment}

The CAI at CASs 02-09-48, 03-09-02, 12-09-01, 12-09-08, and 12-30-14 involved visual inspections and soil sampling (sediment and liquid sampling for CAS 12-30-14) adjacent to and/or beneath debris identified as potential sources for contaminant releases. The CAI results indicate that liquid and sediment are present in the cellar; however, the analytical results for the liquid and the underlying sediment indicate no contamination is present for potential release. Analytical results for all other CASs indicate that no COCs are present.

The maximum concentration of contaminants identified at each CAS, and their corresponding PALs, are presented in Tables 2-1 through 2-6.

\section{C.1.3 C. Site Classification and Initial Response Action}

The four major site classifications listed in Table 3 of the ASTM Standard are (1) immediate threat to human health, safety, and the environment; (2) short-term (0 to 2 years) threat to human health, safety, and the environment; (3) long-term (greater than 2 years) threat to human health, safety, or the environment; and (4) no demonstrated long-term threats. 
Based on the CAI, none of the CASs present an immediate threat to human health, safety, and the environment; therefore, no interim response actions are necessary at these sites. Based on this information, all five CASs are determined to be Classification 4 sites as defined by ASTM Method E 1739-95 (ASTM, 1995) and pose no demonstrated near- or long-term threats.

\section{C.1.4 D. Development of Tier I Lookup Table of Risk-Based Screening Levels}

Tier I action levels have been defined as the PALs established during the DQO process. The PALs are a tabulation of chemical-specific (but not site-specific) screening levels based on the type of media (soil) and potential exposure scenarios (industrial). These are very conservative estimates of risk, are preliminary in nature, and are used as action levels for site screening purposes. Although the PALs are not intended to be used as FALs, a FAL may be defined as the Tier I action level (i.e., PAL) value if individual contaminant analytical results are below the corresponding Tier I action level value. The FAL may also be established as the Tier I action level value if individual contaminant analytical results exceed the corresponding Tier I action level value and implementing a corrective action based on the FAL is practical. The PALs are defined as:

- The U.S. Environmental Protection Agency (EPA) Region 9 Risk-Based Preliminary Remediation Goals (PRGs) for Industrial Soils (EPA, 2004).

- Background concentrations for RCRA metals will be evaluated when natural background exceeds the PAL, as is often the case with arsenic. Background is considered the mean plus two times the standard deviation of the mean based on data published in Mineral and Energy Resource Assessment of the Nellis Air Force Range (NBMG, 1998; Moore, 1999).

- The TPH concentrations above the action level of $100 \mathrm{mg} / \mathrm{kg}$ per NAC 445A.2272 (NAC, 2006c).

- For COPCs without established PRGs, a protocol similar to EPA Region 9 will be used to establish an action level; otherwise, an established PRG from another EPA region may be chosen.

- The PALs for material, equipment, and structures with residual surface contamination are the allowable total residual surface contamination values for unrestricted release of material and equipment listed in the DOE Order 5400.5 (DOE, 1993), which is also Table 4-2 of the NV/YMP Radiological Control Manual (NNSA/NSO, 2004).

- The PALs for radioactive contaminants are based on the National Council on Radiation Protection and Measurements (NCRP) Report No. 129 recommended screening limits for 
construction, commercial, industrial land-use scenarios (NCRP, 1999) scaled to 25-millirem-per-year dose constraint (Appenzeller-Wing, 2004) and the generic guidelines for residual concentration of radionuclides in DOE Order 5400.5 (DOE, 1993).

The PALs were developed based on an industrial scenario. Because the CAU 234 CASs are not assigned work stations and are considered to be in remote or occasional use areas, the use of industrial reuse based PALs is conservative. The Tier I lookup table is defined as the PAL concentrations or activities defined in the CAIP (NNSA/NSO, 2007).

\section{C.1.5 E. Exposure Pathway Evaluation}

The DQOs stated that site workers would only be exposed to COCs through oral ingestion, inhalation, or dermal contact (absorption) due to exposure to potentially contaminated media (i.e., soil) at the CASs. The results of the CAI showed that no COCs are present at CASs within CAU 234. Because no COCs were identified at any of the CASs no potential exposure pathways exist.

\section{C.1.6 F. Comparison of Site Conditions with Tier I Risk-Based Screening Levels}

All analytical results from CAU 234 samples were less than corresponding Tier 1 action levels (i.e., RBSLs).

\section{C.1.7 G. Evaluation of Tier I Results}

For all contaminants at all CASs, the FALs were established as the Tier 1 RBSLs. It was determined that no further action is required at these CASs.

\section{C.1.8 H. Tier I Remedial Action Evaluation}

The corrective action alternative of no further action was implemented based on Tier I RSBL.

\section{C.1.9 I. Tier II Evaluation}

Because no analytes were identified in any of the CASs above their respective FALs, no Tier II evaluations are necessary. 


\section{C.2.0 Recommendations}

As all of the site contaminant concentrations in soils from the analysis of CAU 234 samples were less than the corresponding FALs at all locations, and because the liquid and sediment at CAS 12-30-14 do not pose as PSM, it was determined that there is no significant risk to human health or the environment. No COCs were identified at any of the CASs in CAU 234; therefore, no corrective action is necessary. However, this does not preclude the consideration of these sites for additional protective measures that will be implemented as BMPs.

Based on the analytical results of all samples collected from the investigation of CAU 234, no corrective actions are required. 


\section{C.3.0 References}

ASTM, see American Society for Testing and Materials.

American Society for Testing and Materials. 1995. Standard Guide for Risk-Based Corrective Action Applied at Petroleum Release Sites, ASTM E 1739-95 (Reapproved 2002). Philadelphia, PA.

Appenzeller-Wing, J., U.S. Department of Energy, National Nuclear Security Administration Nevada Site Office. 2004. Letter to T.A. Maize (NDEP) entitled, "Submittal of Proposed Radiological Preliminary Action Levels (PALs) for the Industrial Sites Project," 15 January. Las Vegas, NV.

DOE, see U.S. Department of Energy.

EPA, see U.S. Environmental Protection Agency.

Moore, J., Science Applications International Corporation. 1999. Memorandum to M. Todd (SAIC), "Background Concentrations for NTS and TTR Soil Samples," 3 February. Las Vegas, NV.

NAC, see Nevada Administrative Code.

NBMG, see Nevada Bureau of Mines and Geology.

NCRP, see National Council on Radiation Protection and Measurements.

NNSA/NSO, see U.S. Department of Energy, National Nuclear Security Administration Nevada Site Office.

National Council on Radiation Protection and Measurements. 1999. Recommended Screening Limits for Contaminated Surface Soil and Review of Factors Relevant to Site-Specific Studies/National Council on Radiation Protection and Measurements, NCRP Report No. 129. Bethesda, MD.

Nevada Administrative Code. 2006a. NAC 445A.227, "Contamination of Soil: Order by Director for Corrective Action; Factors To Be Considered in Determining Whether Corrective Action Required." Carson City, NV. As accessed at http://www.leg.state.nv.us/nac on 22 January 2008.

Nevada Administrative Code. 2006b. NAC 445A.22705, "Contamination of Soil: Evaluation of Site by Owner or Operator; Review of Evaluation by Division." Carson City, NV. As accessed at http://www.leg.state.nv.us/nac on 22 January 2008.

Nevada Administrative Code. 2006c. NAC 445A.2272, "Contamination of Soil: Establishment of Action Levels." Carson City, NV. As accessed at http://www.leg.state.nv.us/nac on 22 January 2008. 
Nevada Bureau of Mines and Geology. 1998. Mineral and Energy Resource Assessment of the Nellis Air Force Range, Open-File Report 98-1. Reno, NV.

U.S. Department of Energy. 1993. Radiation Protection of the Public and the Environment, DOE Order 5400.5, Change 2. Washington, DC.

U.S. Department of Energy, National Nuclear Security Administration Nevada Site Office. 2004. NV/YMP Radiological Control Manual, Rev. 5, DOE/NV/11718--079, UC-702. Prepared by Bechtel Nevada. Las Vegas, NV.

U.S. Department of Energy, National Nuclear Security Administration Nevada Site Office. 2006. Industrial Sites Project Establishment of Final Action Levels, Rev. 0, DOE/NV--1107.

Las Vegas, NV.

U.S. Department of Energy, National Nuclear Security Administration Nevada Site Office. 2007. Corrective Action Investigation Plan for Corrective Action Unit 234: Mud Pits, Cellars, and Mud Spills, Nevada Test Site, Nevada, Rev. 0, DOE/NV--1216. Las Vegas, NV.

U.S. Environmental Protection Agency. 2004. Region 9 Preliminary Remediation Goals (PRGs). As accessed at http://www.epa.gov/region09/waste/sfund/prg/index.htm on 22 January 2008. 


\section{Appendix D}

\section{Closure Activity Summary}




\section{D.1.0 Closure Activity Summary}

Closure activities were not required at any of the CASs of CAU 234; however, debris was removed as a BMP.

Debris was removed from four of the five CASs sampled during the investigation. Below is a list, by CAS, of the nonhazardous, nonradioactive, and nonhydrocarbon debris identified for removal and disposal (with reference to the associated photographs of the "before" and "after" removal of the debris):

- CAS 02-09-48: One partially rusted-out 55-gal empty metal drum that was located within the drilling mud sump, then staged for removal. (Waste Item \#234AD1) (Figures D.1-1 and D.1-2).

- CAS 03-09-02: Four pieces of loose empty metal and a polyvinyl chloride (PVC) pipe section from the suction pit in the northern footprint of the CAS (Waste Item \#234BP1) (Figures D.1-3 and D.1-4).

- CAS 12-09-01: One empty metal pipe, approximately $20 \mathrm{ft}$ in length (Waste Item \#234CP1), and one metal cylinder, approximately 4 by $8 \mathrm{ft}$ and weighing approximately 250 pounds, that appears to have been converted into a trash incinerator (Waste Item \#234CC1) (Figures D.1-5 through D.1-7); glass and metal trash from inside the cylinder (Waste Item \#234CT1) (not shown in figures).

- CAS 12-09-08: One 4-ft section of empty metal pipe (Waste Item \#234DP1), two crushed (empty) 30-gal metal open-top drums (Waste Items \#234DD1 and \#234DD2), one 55-gal crushed metal closed-top drum (Waste Item \#234DD3), one rusted drum (Waste Item \#234DD4), one motor vehicle exhaust pipe (Waste Item \#234DP2) (Figures D.1-8 through D.1-11).

The debris has been characterized as nonhazardous and nonradioactive and was disposed of in the Area 9 U10c Industrial Landfill. The waste disposal form to document this effort is included as Attachment D-1. Although four separate disposal forms were generated, one for each CAS, all the waste was loaded to and transported in the same vehicle, so acceptance and disposal of all waste was executed on the first disposal document only. 


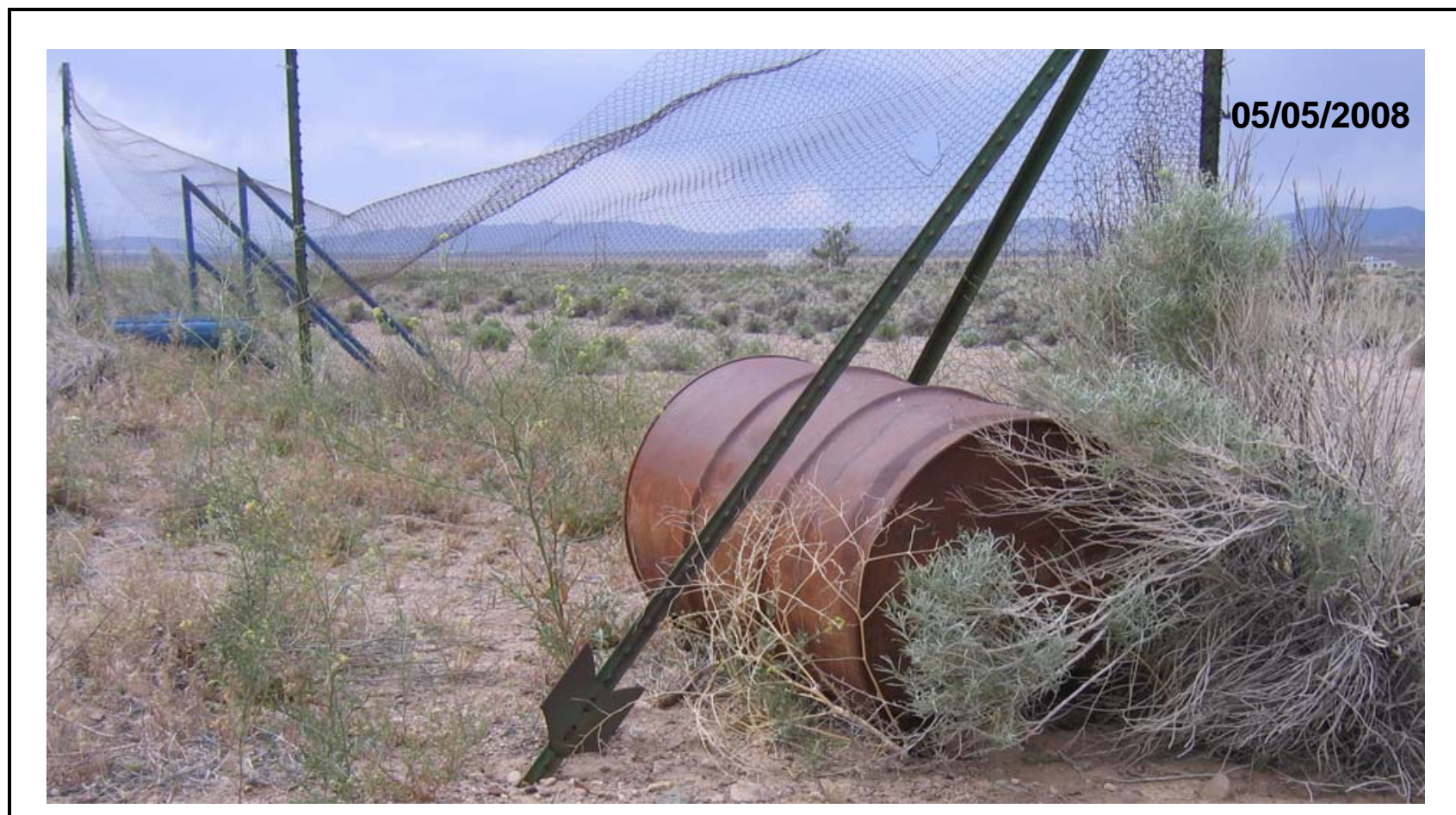

Figure D.1-1

Debris at CAS 02-09-48

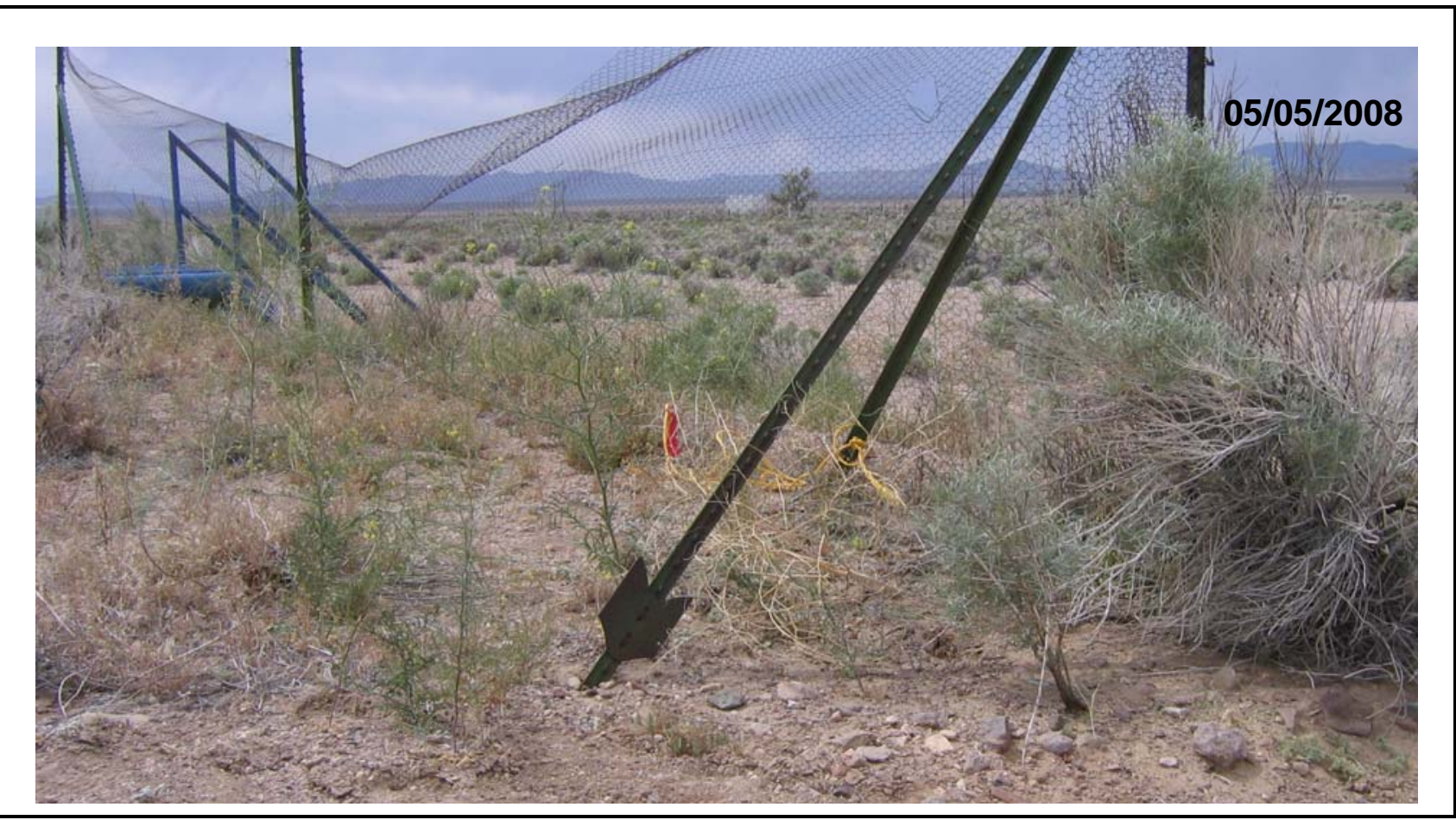

Figure D.1-2

Area after Debris Removal at CAS 02-09-48 


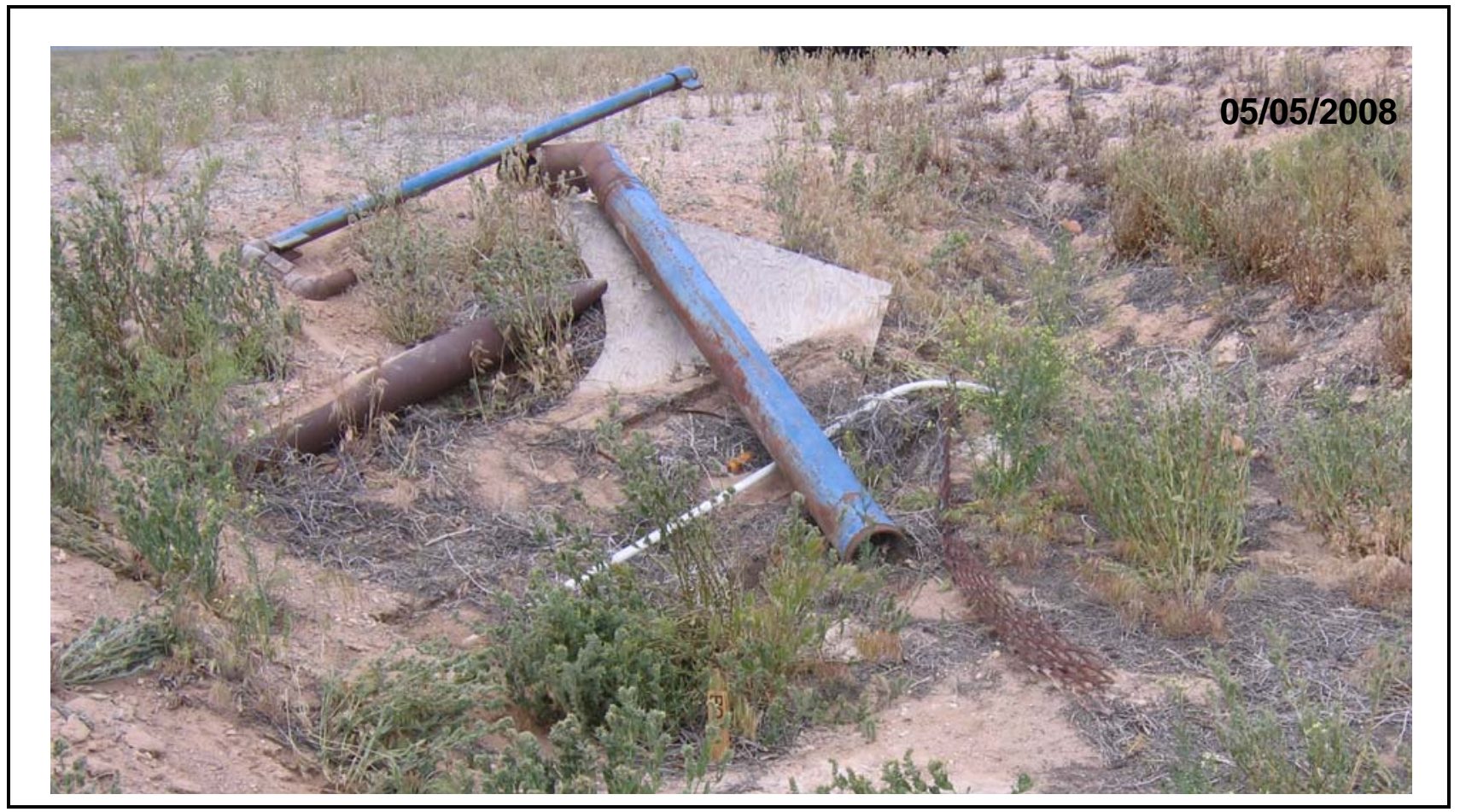

Figure D.1-3

Debris at CAS 03-09-02

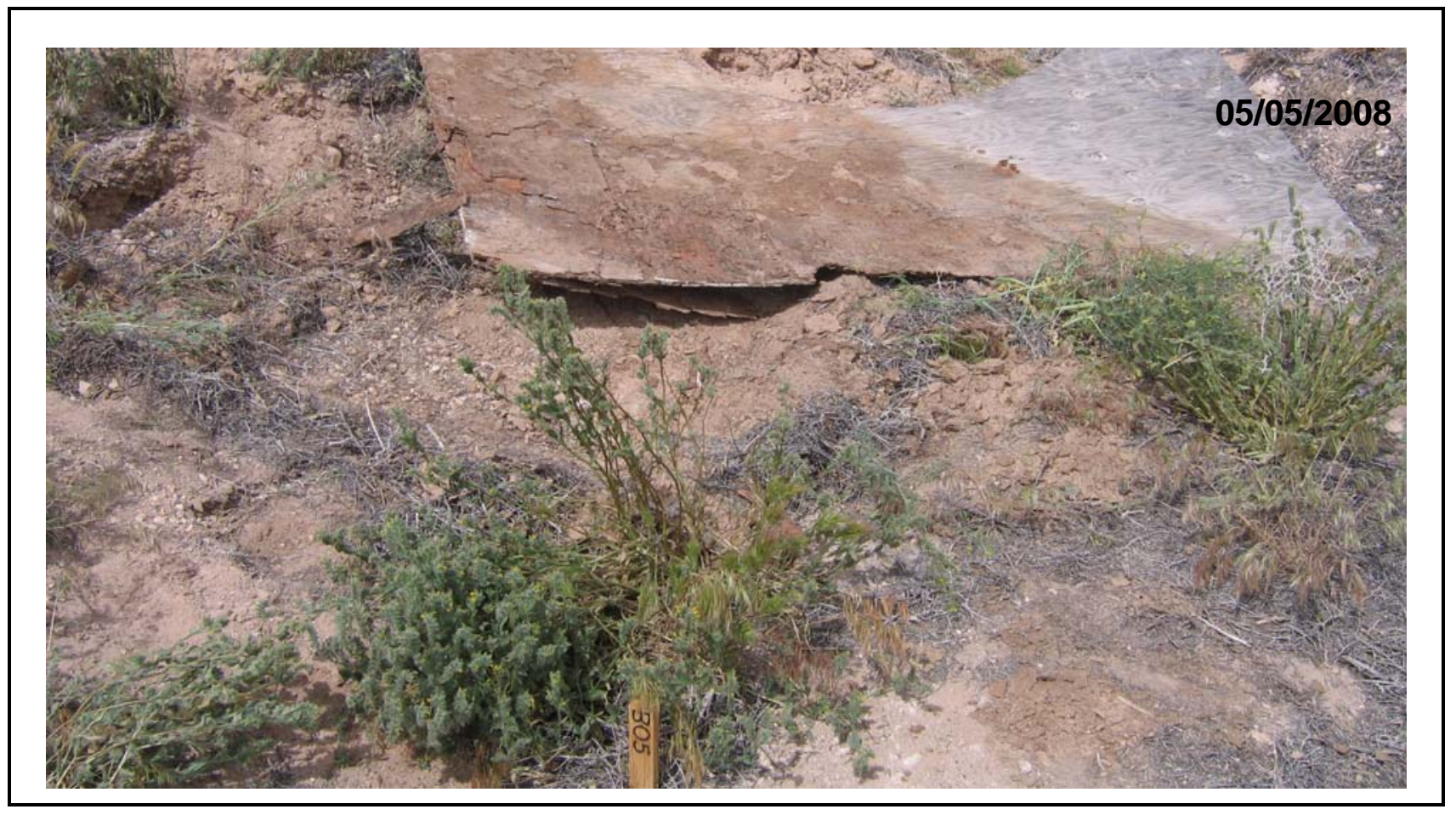

Figure D.1-4

Area after Debris Removal at CAS 03-09-02 


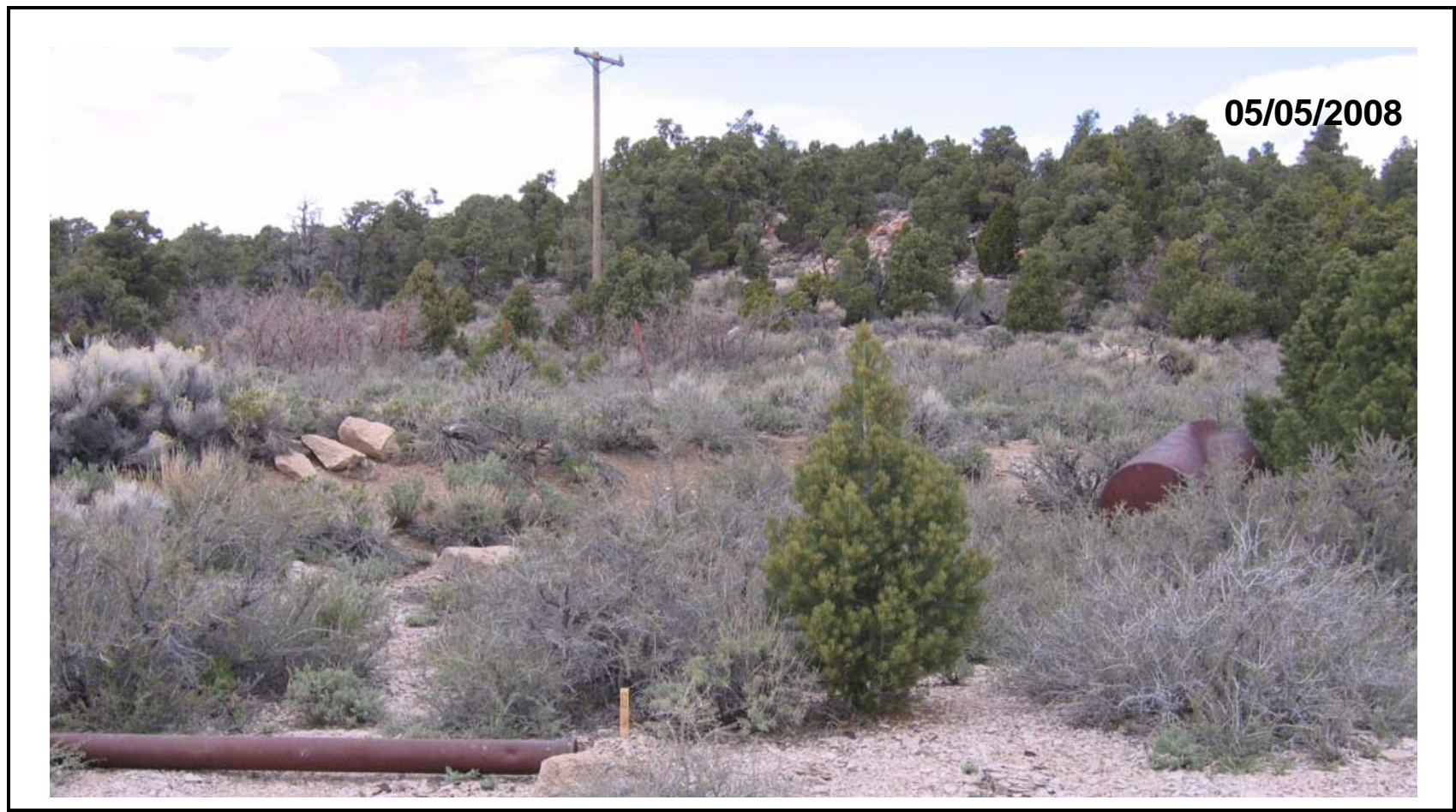

Figure D.1-5

Debris at CAS 12-09-01

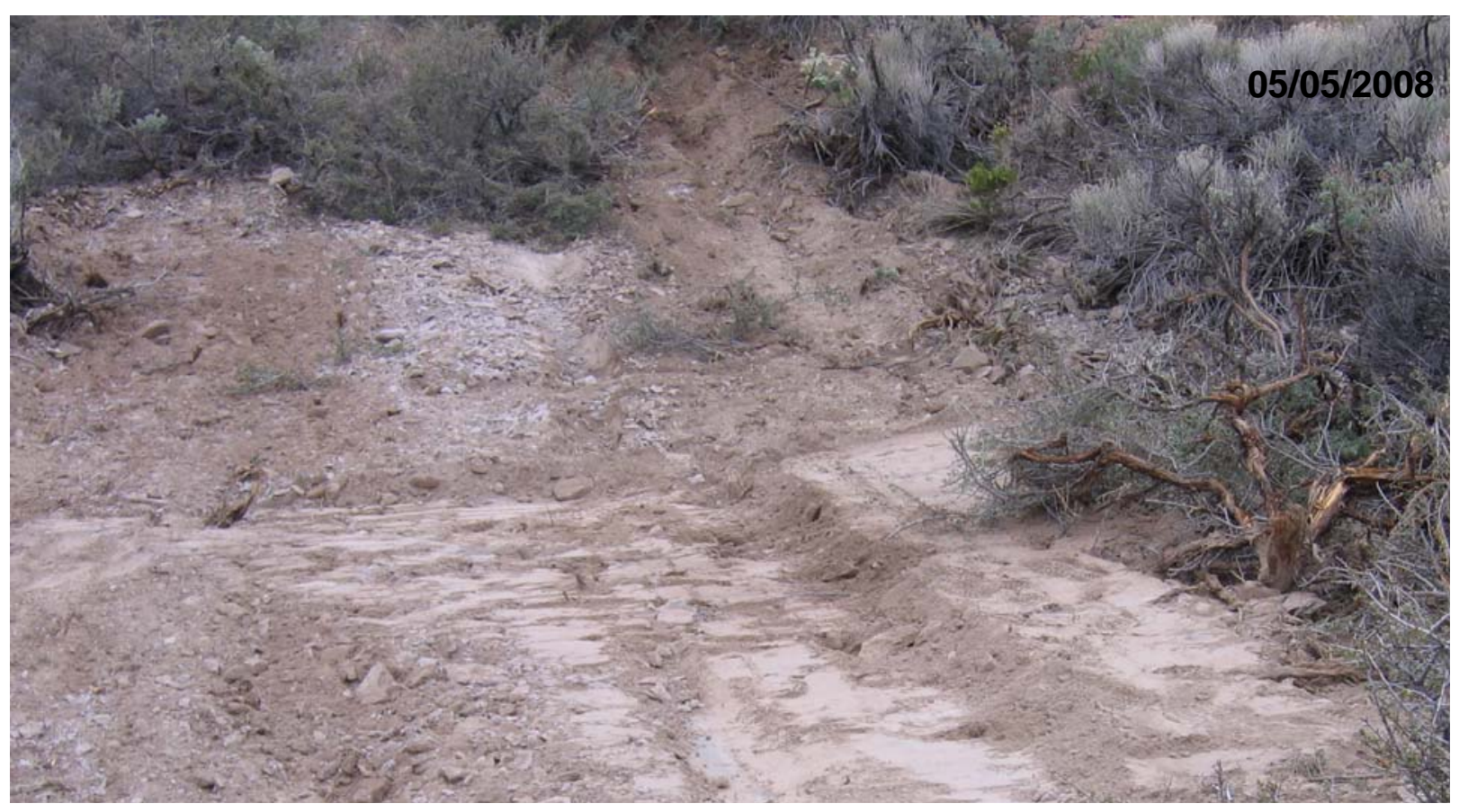

Figure D.1-6

Area after Pipe Debris Removal at CAS 12-09-01 


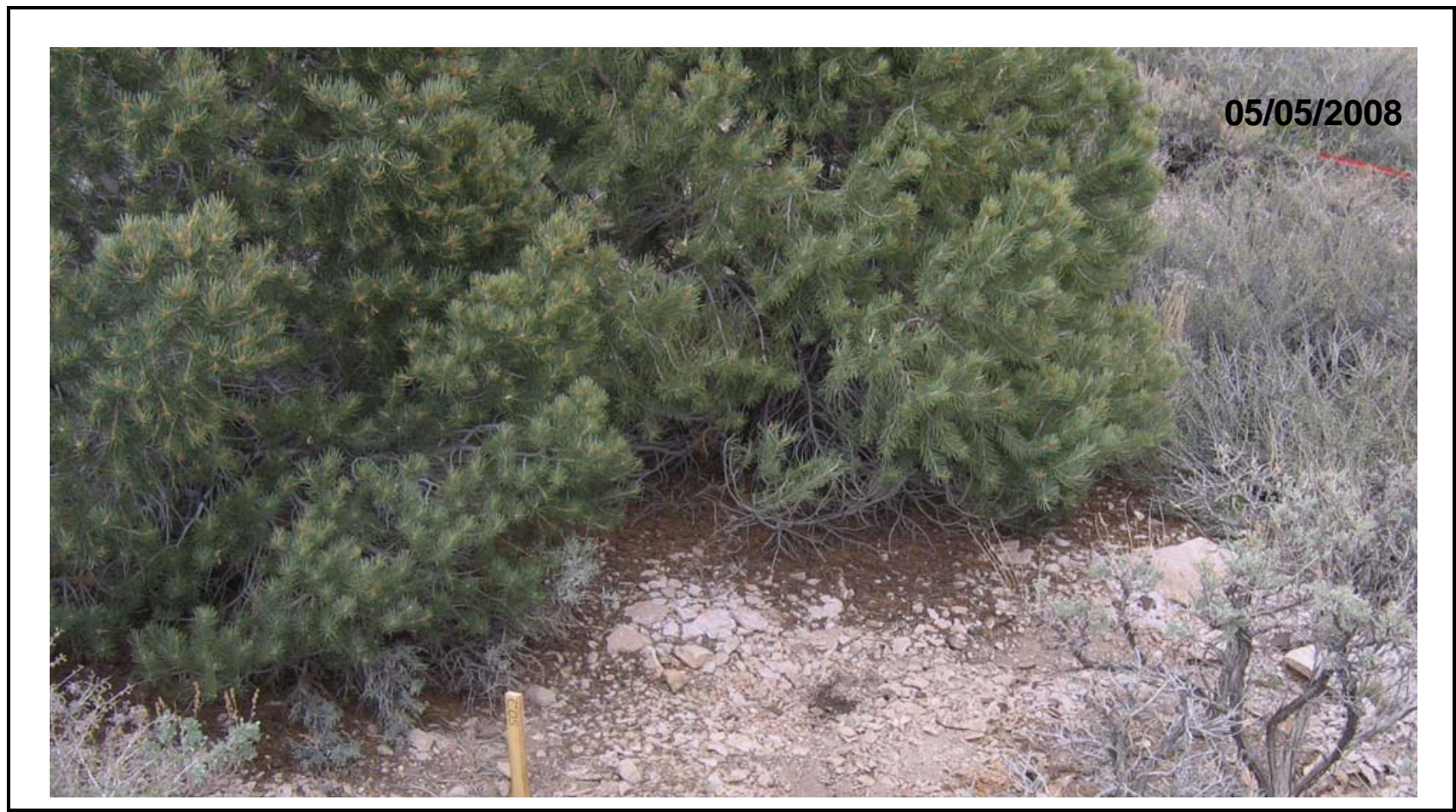

Figure D.1-7

Area after Cylinder Debris Removal at CAS 12-09-01

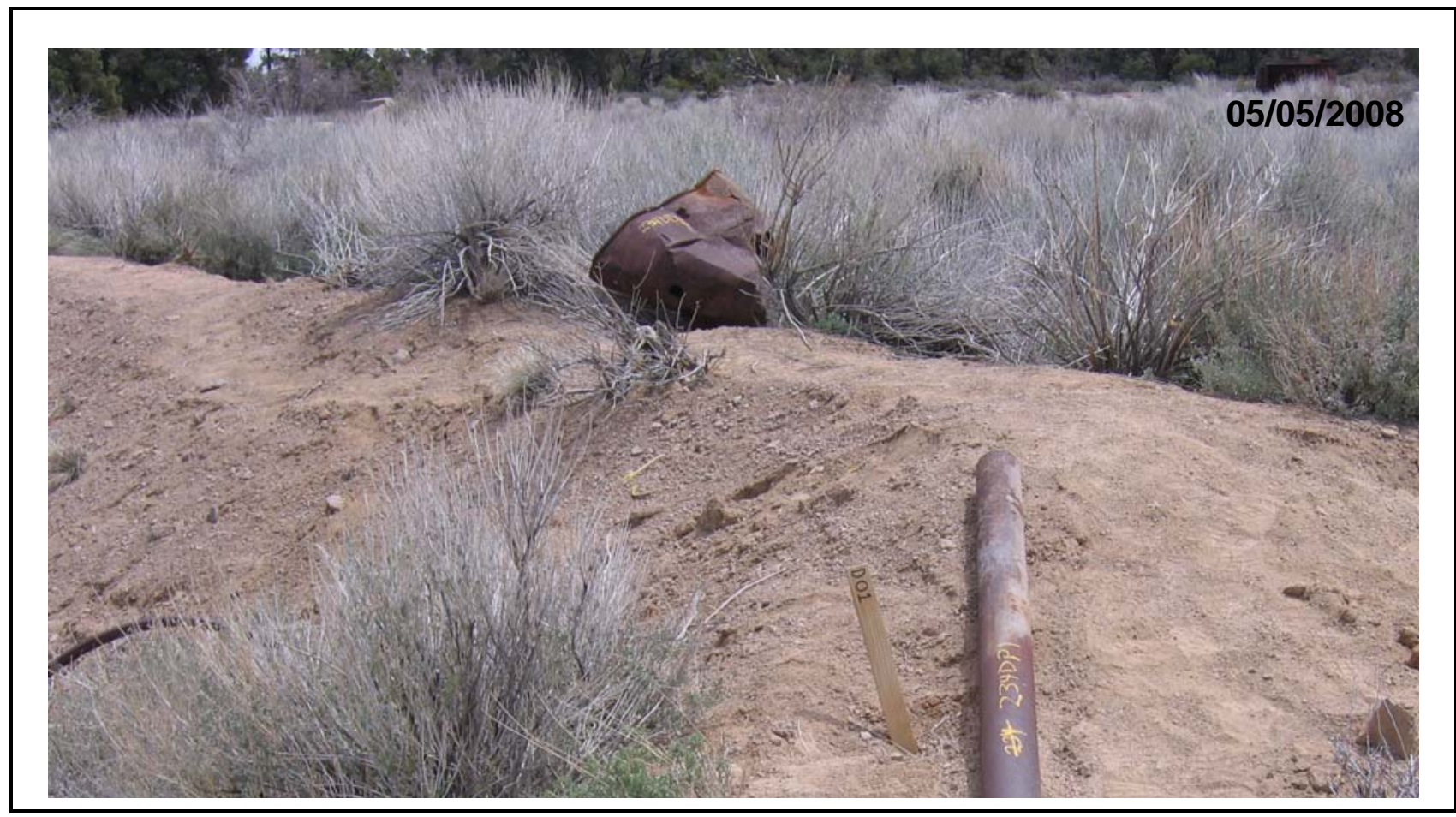

Figure D.1-8

Debris at CAS 12-09-08 


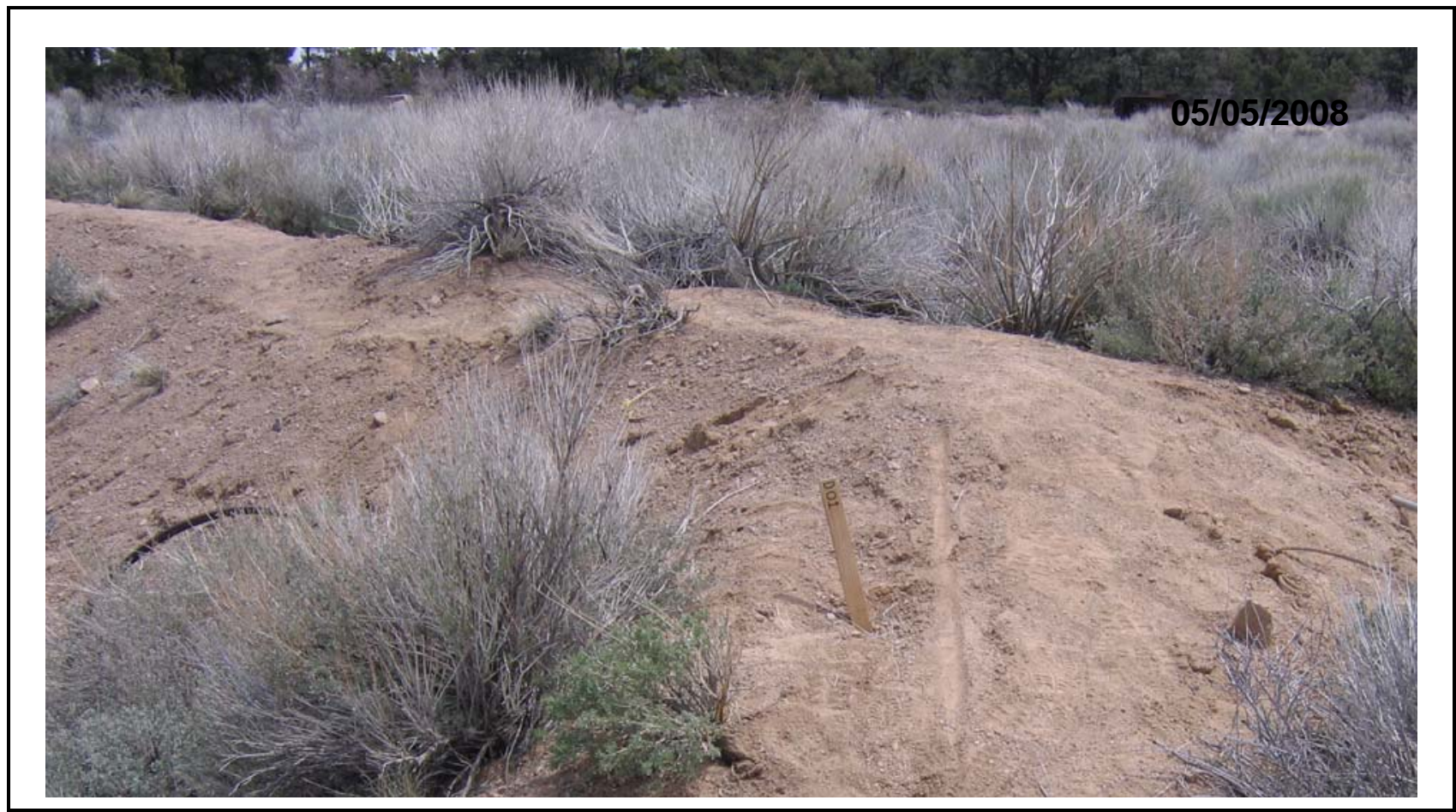

Figure D.1-9

Area after Pipe and Drum Debris Removal at CAS 12-09-08

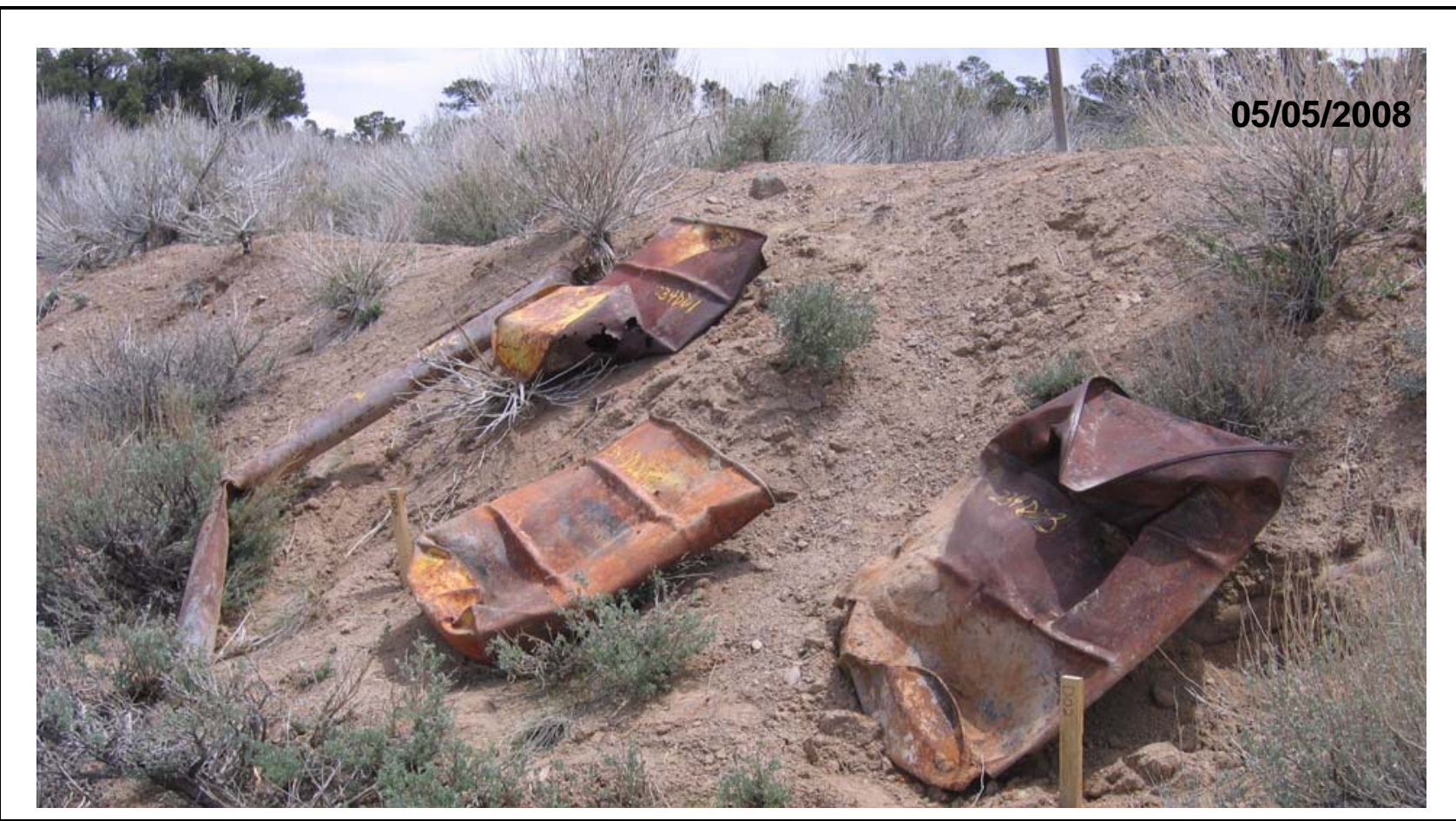

Figure D.1-10

Debris at CAS 12-09-08 


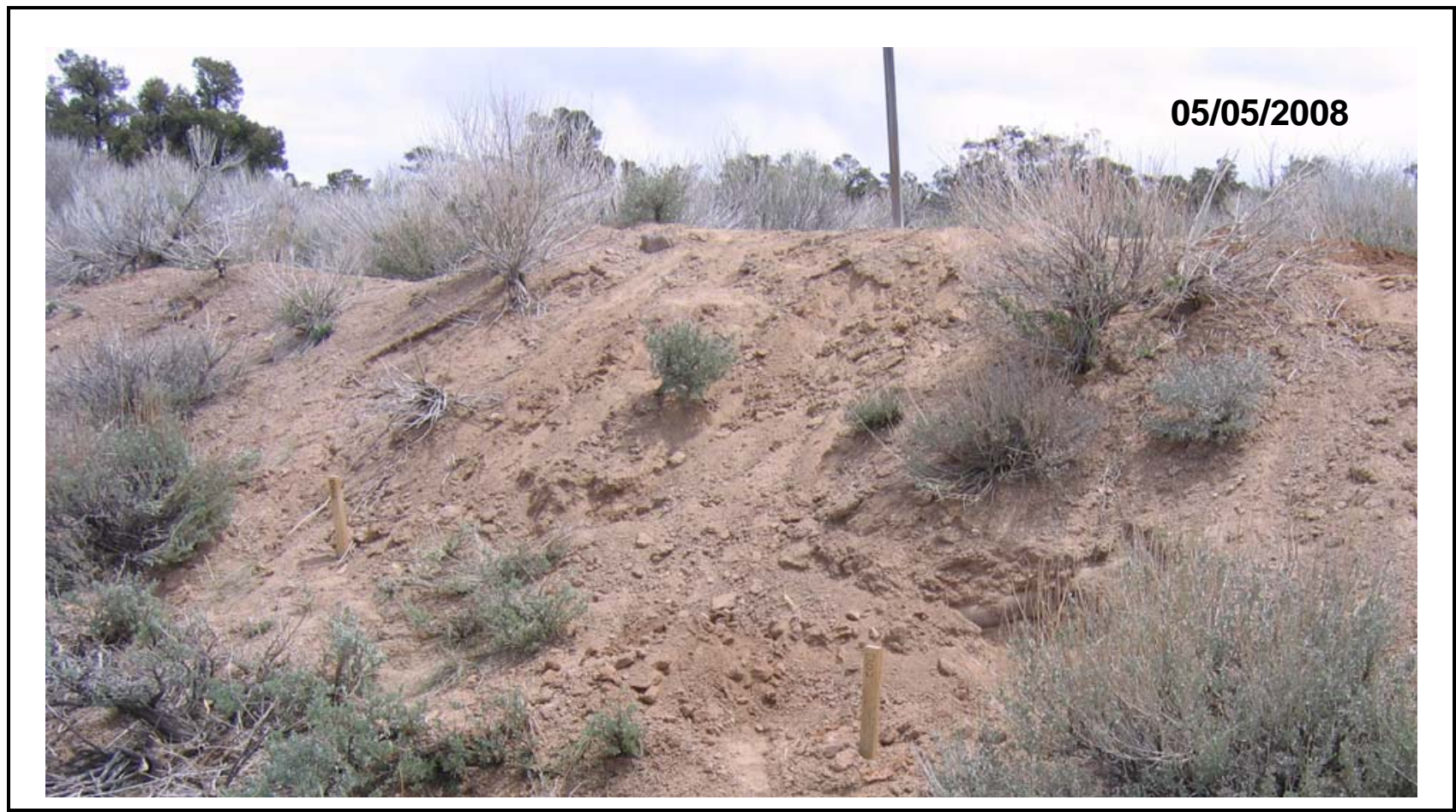

Figure D.1-11

Area after Drum Debris Removal at CAS 12-09-08 


\section{Attachment D-1}

\section{Load Verification Forms}

(1 Page) 
NSTeC

Form

FRM-0918

NTS LANDFILL LOAD VERIFICATION

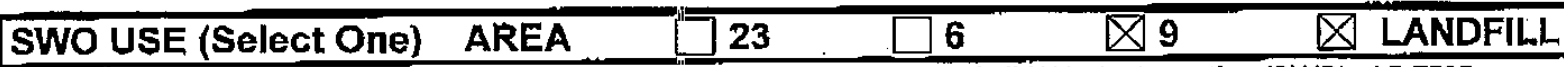

For waste characterization, approval, andior assistance, contact Solid Waste Operation (SWO) at 5-7898. REQUIRED: W'ASTE GERERATOR INFORMATION

(This form is for rolloffs, dump trucks, and other ansite disposal of materials.)

Waste Generator. Rene Robles (SNJV, WO)

Phone Number: 5-2100

Location / Origin: CAU 234. CAS 03-09-02, Area j- 4, pieces of loose empty metal \& PVC pipes (WastelD\#234BP1)

\begin{tabular}{|c|c|c|c|c|}
\hline \multicolumn{2}{|c|}{ Waste Category: (check one) } & \multirow{2}{*}{$\begin{array}{l}\square \text { Conmercial } \\
\square \text { Puirescrible }\end{array}$} & \multicolumn{2}{|l|}{ 凶 Industrial } \\
\hline Waste Type: & $\square$ NTS & & 区 FFACO-onsite & \\
\hline (check one) & $\square$ Non-Putrescible & $\square$ Asbestos Containing Material & $\square$ FFACO-offsite & [] Historic DOE/NV \\
\hline \multicolumn{2}{|c|}{ Pollution Prevention Category: (check one) } & $\triangle$ Environmental management & $\square$ Defense Projects & $\square Y M P$ \\
\hline \multicolumn{2}{|c|}{ Pollution Prevention Category: (check one) } & $\Delta$ Clean-Up & $\square$ Routine & \\
\hline \multicolumn{2}{|c|}{ Method of Characterization: (check one) } & Х Sampling \& Analysis & $\triangle$ Process Knowledge & $\square$ Contents \\
\hline
\end{tabular}

Prohibited Waste at all three Radioactive waste; RCRA waste; Hazardous waste; Free liquids, PCBs above TSCA regulatory NTS landfills:

Additional. Prohibited Waste at the Area 9 U10C Landfill: levels, and Medical wastes (needles, sharps, bloody clothing)

Sewage Sludge, Animal carcasses, Wet garbage (food waste); and Friable asbestos

REQUIRED: WASTE CONTENTS ALLOWABLE WASTES

Check all allowable wastes that are contained within this load:

NOTE: Waste disposal at the Area 6 Hydrocarbon Landfill must have come into contact with petroleum hydrocarbons or coolants, such as: gasoline (no benzene, lead); jet fuel; diesel fuel; lubricants and hydraulics; kerosene; asphaltic petrolesm hydrocarbon; and ethylene glycol.
Acceptable waste at any NTS landfill:
I] Paper
$\square$ Rocks / unaltered geologic materials
$\square$ Empty containers
$\square$ Asphalt $\quad$ Metal $\square$ Wood
$\square$ Soil
$\square$ Rubber (excluding tires)
$\square$ Demolition debris
Q Plastic
$\square$ Cable
$\square$ Cloth
$\square$ Insulation (non-Asbestosform)
$\square$ Cement \& concrete

$\square$ Manufactured items: (swamp coolers, furnizure, rugs, carpet, electronic components, PPE, etc.)
Additional waste accepted at the Area 23 Mercury Landfill: $\square$ Office Waste
$\square$ Food Waste
Animal Carcasses

$\square$ Asbestos $\square$ Friable

$\square$ Non-Friable (contact SWO if regulated load)

Additional waste accepted at the Area 9 U10c Lanidfill:
$\square$ Non-friable asbestos
$\square$ Drained automobiles and military vehicles
$\square$ Non-friable asbestos
$\square$ Light ballasts (contact swo)
$\square$ Hydrocarbons (contaet swo)
$\square$ Drained fuel filtors (gas \& diesel)
Solid fractions from sand/oil/water
Other
$\square$ Deconned Underground and Above Ground Tanks

Quantity:

\begin{tabular}{|c|c|c|c|}
\hline \multicolumn{4}{|c|}{ Additional waste accepted at the Area 6 Hydrocar'bon Landfill: $\square$} \\
\hline$\square$ Septic sludge & $\square$ Rags & $\square$ Drained fuel filters (gas \& diesel) & $\square$ Crushed non-teme plated oil filters \\
\hline$\square$ Plants & $\square$ Soill & $\square$ Sluegge frem sand/oil/water separators & PCBs below 50 parts per million \\
\hline
\end{tabular}

tnitials: (If initialed, no radiological clearanice is necessary.)

The above mentioned waste was generated outside of a Controllod Waste Managernent Area (CWMA) and to the best of my knowledge, does not contain radlologlcal materials.

To the best of my knowledge, the waste described aljove contains only those site. I have verified this through the waste characterization method identified prohibited and allowable waste items. I have contaciled Property Management is approved for disposal in the landfill.

Print Name: Joe Hdotrov

Signature:

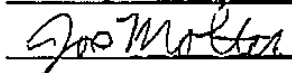<smiles>CC(C)(C)[As]1C=CC=C1</smiles>

Radlological Survey Release for Wasto Dlsposal RCT Inltlals:

This containar/load meets the erlterla for no addod man-made radlosctive material Thlo containerilosd meots the criterla for Radicon Manual Tabla 4.2 reloase Ilmits. This contalner/load ls exempt from survey dur to process knowleoge and origin.

Note: "Food waste, office trash and animal carcasse:s do not require a radiolog must have signed removal certification statemunt with Load Verification.

SWO USE ONLY

Load Weight (net frogn scale or estimate): $20 \mathrm{Cl}$
$5 / 6 / 08$ Signature of Certifiec
SITATUR:

SIGNATURE: 


\section{Appendix E}

\section{Sample Location Coordinates}




\section{E.1.0 Sample Location Coordinates}

Sampling location coordinates for the CAI sampling were determined using a Trimble Geo-XT GPS unit. The CAU 234 Decision I sampling locations are presented with easting and northing coordinates in Figures E.1-1 through E.1-5. 


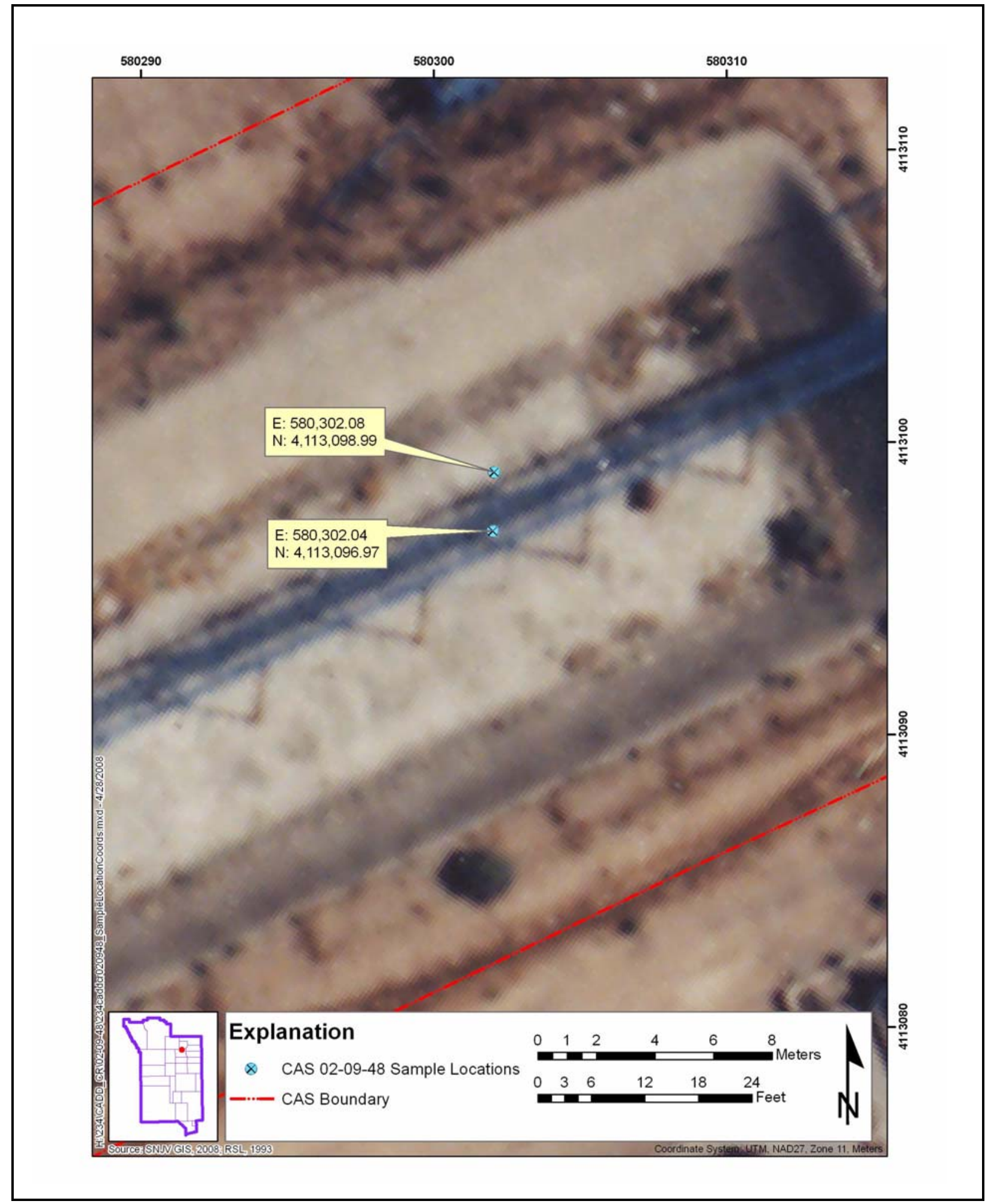

Figure E.1-1

Sample Location Coordinates for CAS 02-09-48 


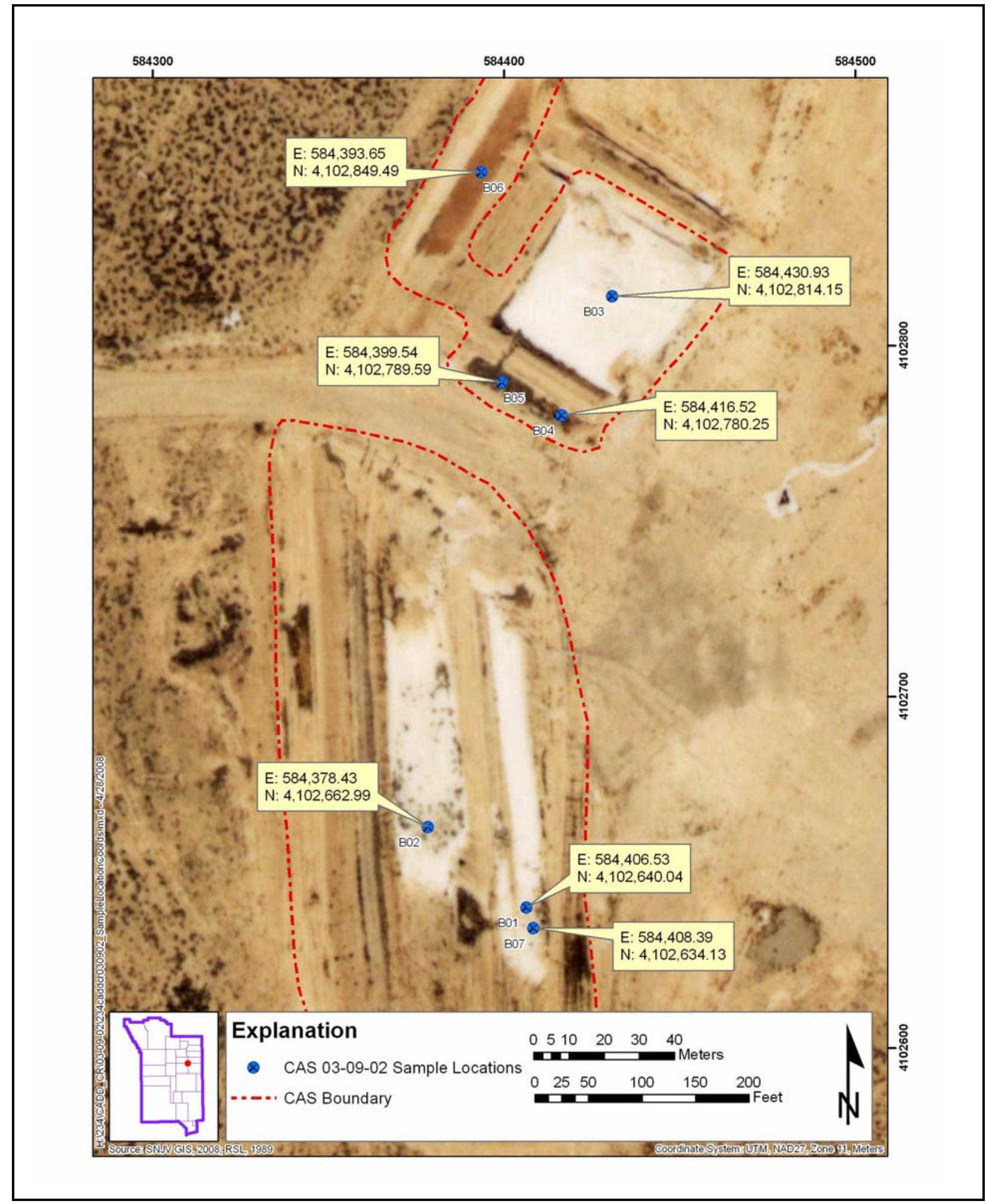

Figure E.1-2

Sample Location Coordinates for CAS 03-09-02 


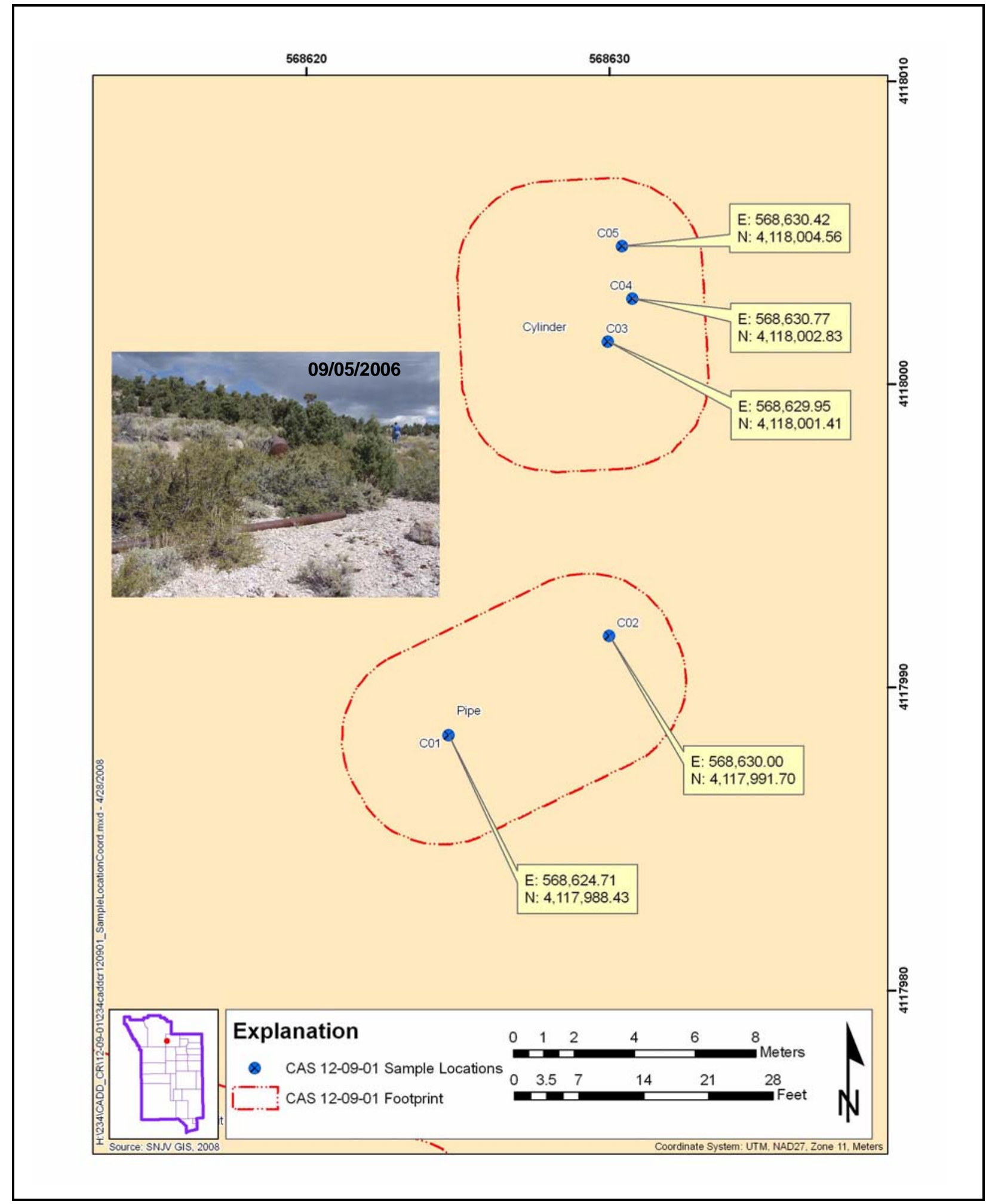

Figure E.1-3

Sample Location Coordinates for CAS-12-09-01 


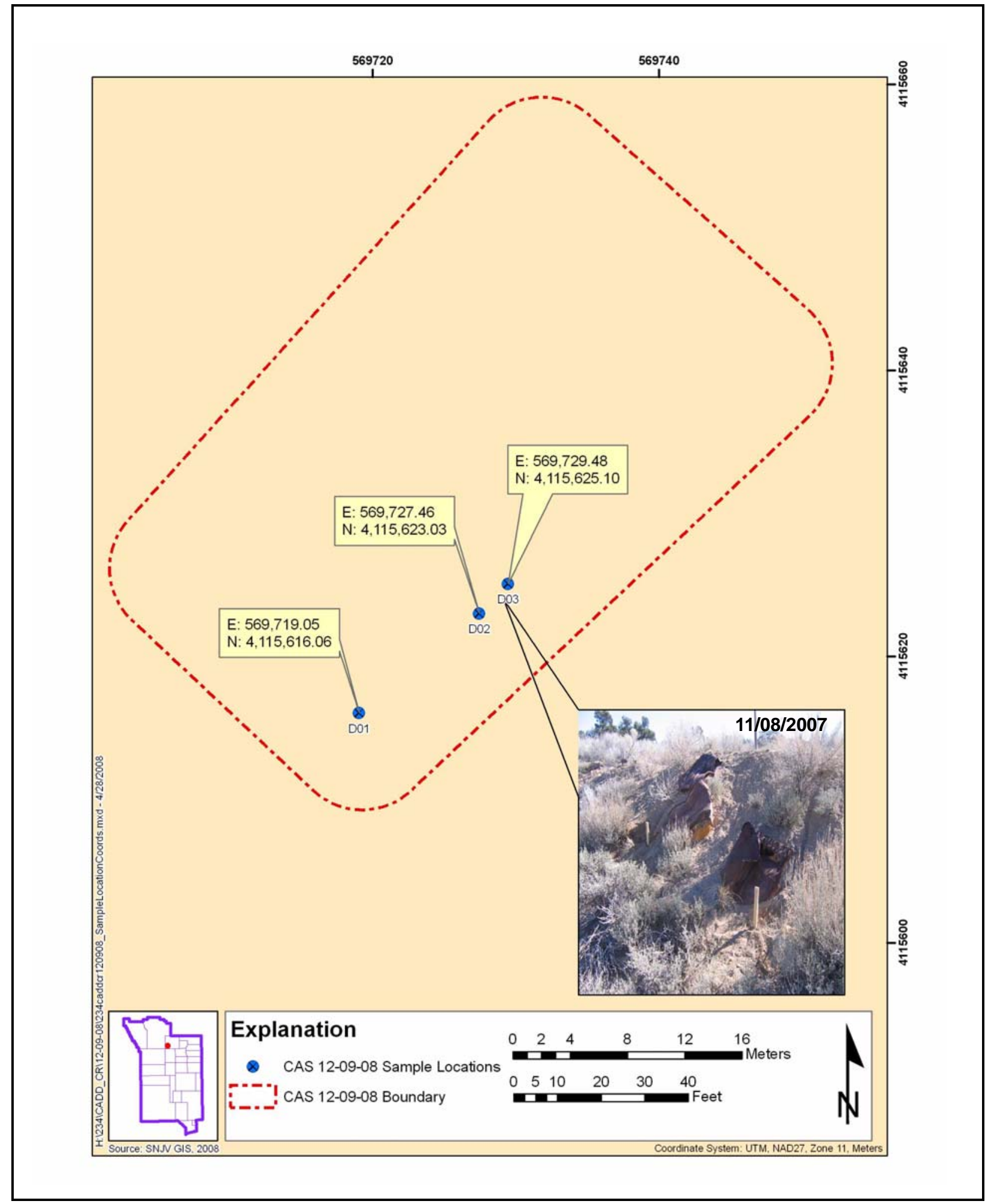

Figure E.1-4

Sample Locations Coordinates for CAS 12-09-08 


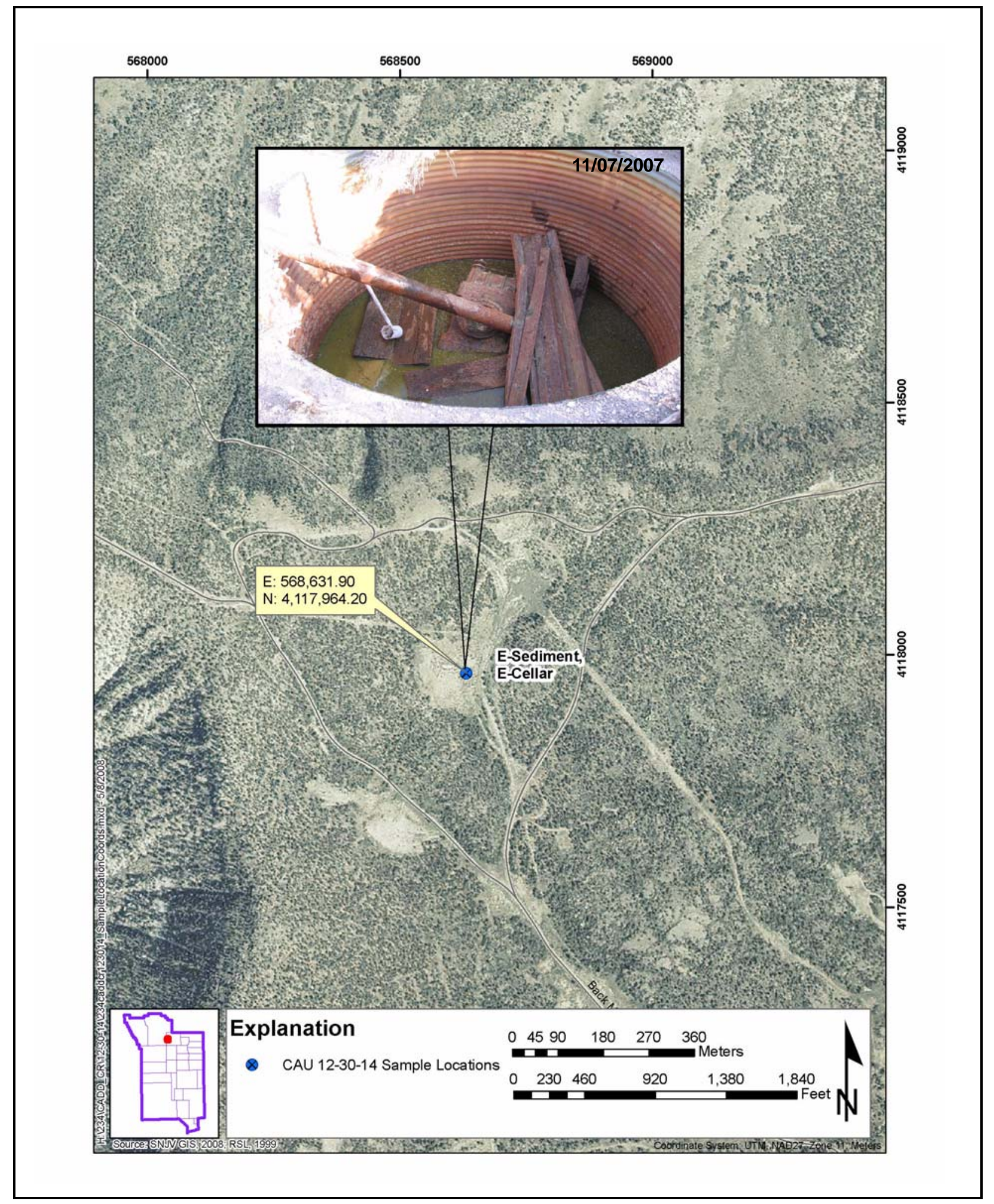

Figure E.1-5

Sample Location Coordinates for CAS 12-30-14 


\section{E.2.0 References}

RSL, see Remote Sensing Laboratory.

Remote Sensing Laboratory. 1989. Aerial photograph “6612-146.” Las Vegas, NV.

Remote Sensing Laboratory. 1993. Aerial photograph “7427-04\#2” showing Area 2 mud and crater storage sumps, 1 June. Las Vegas, NV: EG\&G Energy Measurements, Inc.

Remote Sensing Laboratory. 1999. Digital Orthophoto Quarter Quads. Las Vegas, NV.

SNJV GIS, see Stoller-Navarro Joint Venture Geographic Information Systems.

Stoller-Navarro Joint Venture Geographic Information Systems. 2008. ESRI ArcGIS Software. 


\section{Appendix F}

\section{Nevada Division of Environmental Protection Comments}

(2 Pages) 


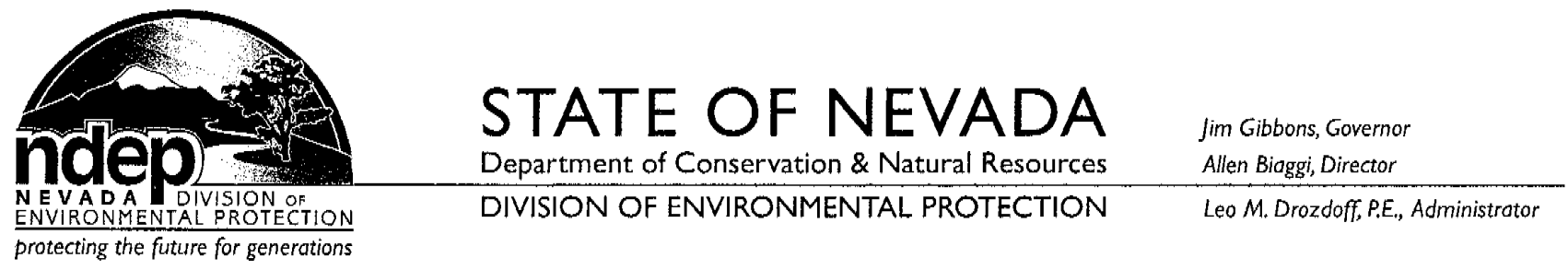

April 18, 2008

Mr. John B. Jones

Acting Federal Project Director, Environmental Restoration Project

National Nuclear Security Administration

Nevada Site Office

P. O. Box 98518

Las Vegas, NV 89193-8518

RE: Review of the draft Corrective Action Decision Document/Closure Report (CADD/CR)

Corrective Action Unit (CAU) 234: Mud Pits, Cellars, and Mud Spills Nevada Test Site, Nevada, Revision 0, Federal Facility Agreement and Consent Order

Dear Mr. Jones:

The Nevada Division of Environmental Protection (NDEP), Bureau of Federal Facilities staff has reviewed the draft Corrective Action Decision Document/Closure Report (CADD/CR) for Corrective Action Unit (CAU) 234: Mud Pits, Cellars, and Mud Spills. After reviewing the summary of investigation activities, sample results, and the submitted analytical data and quality control summaries, NDEP concurs with the proposed corrective action alternative that no further action is required for the 12 sites comprising CAU 234. NDEP does not have any comments regarding the draft CADD/CR. Upon receipt of the final CADD/CR, NDEP will issue a Notice of Completion for the closure of CAU 234.

If you have any questions regarding this matter, please contact me at (702) 486-2850, extension 233 or John Wong at extension 245.

Sincerely,

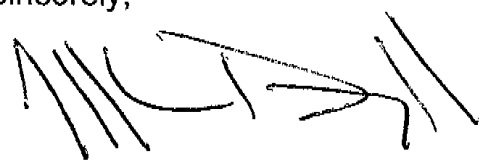

Jeff MacDougall, Ph. D,

Supervisor,

Bureau of Federal Facilities

THM/JJM/jaw 
Mr. Jones

Page 2

April 18, 2008

cc: E.F. DiSanza, WMP, NNSA/NSO

FFACO Group, PSG, NNSA/NSO, Las Vegas, NV

Jeffrey Fraher, DTRA/CXTS, Kirtland AFB, NM

Wayne Griffin, SNJV/DTRA, M/S 645, Mercury, NV

J. A. Ciucci, NSTec, Las Vegas, NV

A. L. Primrose, NSTec, Las Vegas, NV

J. L. Smith, NSTec, Las Vegas, NV

NSTec Technical Information Officer, Las Vegas, NV

K. J. Cabble, ERP, NNSAINSO, Las Vegas, NV

T. A. Thiele, NSTec, Las Vegas, NV

R. F. Boehlecke, SNJV, Las Vegas, NV 


\section{Library Distribution List}

\section{$\underline{\text { Copies }}$}

U.S. Department of Energy

1 (Uncontrolled, electronic copy)

National Nuclear Security Administration

Nevada Site Office

Technical Library

P.O. Box 98518, M/S 505

Las Vegas, NV 89193-8518

U.S. Department of Energy

1 (Uncontrolled, electronic copy)

Office of Scientific and Technical Information

P.O. Box 62

Oak Ridge, TN 37831-0062

Southern Nevada Public Reading Facility

2 (Uncontrolled, electronic copies)

c/o Nuclear Testing Archive

P.O. Box 98521, M/S 400

Las Vegas, NV 89193-8521

Manager, Northern Nevada FFACO

1 (Uncontrolled, electronic copy)

Public Reading Facility

c/o Nevada State Library \& Archives

100 N Stewart Street

Carson City, NV 89701-4285 